University of South Carolina

Scholar Commons

2013

\title{
Applications of High Throughput (Combinatorial) Methodologies to Electronic, Magnetic, Optical, and Energy-Related Materials
}

Martin L. Green

Ichiro Takeuchi

Jason R. Hattrick-Simpers

University of South Carolina - Columbia, simpers@cec.sc.edu

Follow this and additional works at: https://scholarcommons.sc.edu/eche_facpub

Part of the Engineering Physics Commons, Other Chemical Engineering Commons, and the Other Materials Science and Engineering Commons

Publication Info

Published in Journal of Applied Physics, Volume 113, Issue 23, 2013, pages \#231101-.

(C) Journal of Applied Physics 2013, AIP (American Institute of Physics).

Green, M. L., Takeuchi, I., \& Hattrick-Simpers, J. R. (21 June 2013). Applications of High Throughput (Combinatorial) Methodologies to Electronic, Magnetic, Optical, and Energy-Related Materials. Journal of Applied Physics, 113 (23), \#231101. http://dx.doi.org/10.1063/1.4803530

This Article is brought to you by the Chemical Engineering, Department of at Scholar Commons. It has been accepted for inclusion in Faculty Publications by an authorized administrator of Scholar Commons. For more information, please contact digres@mailbox.sc.edu. 


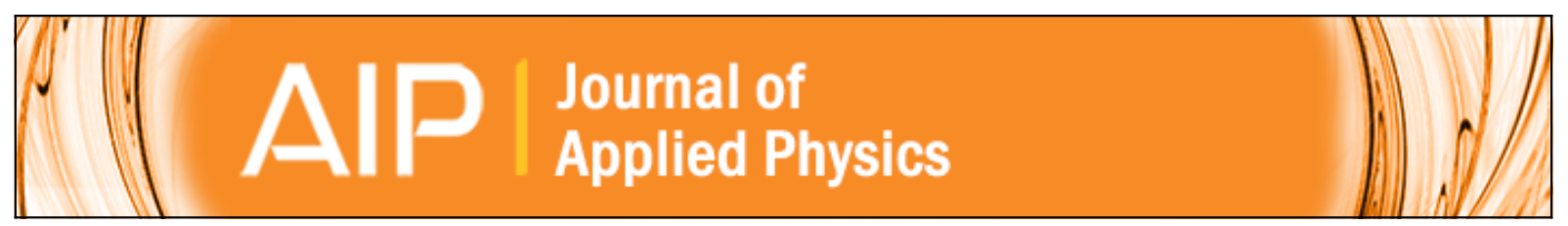

Applications of high throughput (combinatorial) methodologies to electronic, magnetic, optical, and energy-related materials

Martin L. Green, Ichiro Takeuchi, and Jason R. Hattrick-Simpers

Citation: Journal of Applied Physics 113, 231101 (2013); doi: 10.1063/1.4803530

View online: http://dx.doi.org/10.1063/1.4803530

View Table of Contents: http://scitation.aip.org/content/aip/journal/jap/113/23?ver=pdfcov

Published by the AIP Publishing

\section{Articles you may be interested in}

Laguerre-type derivatives: Dobiński relations and combinatorial identities

J. Math. Phys. 50, 083512 (2009); 10.1063/1.3155380

Application of X-ray and Neutron Scattering Techniques in Materials Research: Lithium Batteries and Electronic Ceramics

AIP Conf. Proc. 989, 3 (2008); 10.1063/1.2906087

Generation of High-Gravity Field and Application to Materials Science

AIP Conf. Proc. 973, 455 (2008); 10.1063/1.2896821

Combinatorial Methodologies Applied to the Advanced CMOS Gate Stack

AIP Conf. Proc. 931, 297 (2007); 10.1063/1.2799387

Versatile scanning near-field optical microscope for material science applications

Rev. Sci. Instrum. 68, 3088 (1997); 10.1063/1.1148246

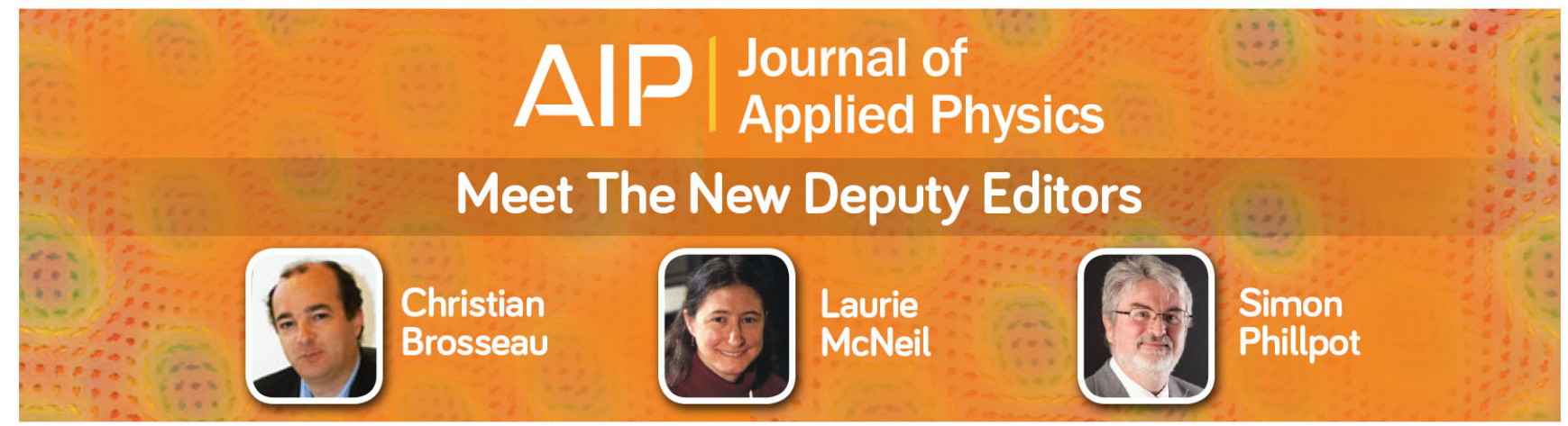




\title{
Applications of high throughput (combinatorial) methodologies to electronic, magnetic, optical, and energy-related materials
}

\author{
Martin L. Green, ${ }^{1}$ Ichiro Takeuchi, ${ }^{2}$ and Jason R. Hattrick-Simpers ${ }^{3}$ \\ ${ }^{1}$ Materials Measurement Laboratory, National Institute of Standards and Technology, Gaithersburg, \\ Maryland 20899, USA \\ ${ }^{2}$ Department of Materials Science and Engineering, University of Maryland, College Park, \\ Maryland 20742, USA \\ ${ }^{3}$ Department of Chemical Engineering, University of South Carolina, Columbia, South Carolina 29208, USA
}

(Received 10 August 2012; accepted 16 April 2013; published online 17 June 2013)

\begin{abstract}
High throughput (combinatorial) materials science methodology is a relatively new research paradigm that offers the promise of rapid and efficient materials screening, optimization, and discovery. The paradigm started in the pharmaceutical industry but was rapidly adopted to accelerate materials research in a wide variety of areas. High throughput experiments are characterized by synthesis of a "library" sample that contains the materials variation of interest (typically composition), and rapid and localized measurement schemes that result in massive data sets. Because the data are collected at the same time on the same "library" sample, they can be highly uniform with respect to fixed processing parameters. This article critically reviews the literature pertaining to applications of combinatorial materials science for electronic, magnetic, optical, and energy-related materials. It is expected that high throughput methodologies will facilitate commercialization of novel materials for these critically important applications. Despite the overwhelming evidence presented in this paper that high throughput studies can effectively inform commercial practice, in our perception, it remains an underutilized research and development tool. Part of this perception may be due to the inaccessibility of proprietary industrial research and development practices, but clearly the initial cost and availability of high throughput laboratory equipment plays a role. Combinatorial materials science has traditionally been focused on materials discovery, screening, and optimization to combat the extremely high cost and long development times for new materials and their introduction into commerce. Going forward, combinatorial materials science will also be driven by other needs such as materials substitution and experimental verification of materials properties predicted by modeling and simulation, which have recently received much attention with the advent of the Materials Genome Initiative. Thus, the challenge for combinatorial methodology will be the effective coupling of synthesis, characterization and theory, and the ability to rapidly manage large amounts of data in a variety of formats. (C) 2013 AIP Publishing LLC. [http://dx.doi.org/10.1063/1.4803530]
\end{abstract}

\section{TABLE OF CONTENTS}

I. INTRODUCTION AND HISTORICAL

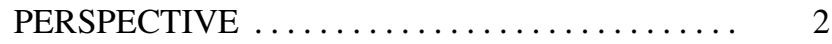

II. ELECTRONIC MATERIALS .............. 2

A. Silicon microelectronics.............. 2

1. Advanced gate stack materials........ 2

III. FERROELECTRIC, PIEZOELECTRIC, AND MULTIFERROIC MATERIALS ........... 10

IV. MAGNETIC MATERIALS................ 13

A. High throughput magnetic characterization techniques ........................ 13

B. Magnetic oxides................. 14

C. Magnetic metallic alloys and magnetic phase diagrams.................... 16

D. Superconductors.................. 22
V. OPTICAL MATERIALS................ 23

A. Thermochromics and electrochromics ..... 23

B. Luminescents and phosphorescents ...... 23

C. Transparent conducting oxides (for photovoltaics)................... 25

1. $\mathrm{In}_{2} \mathrm{O}_{3}$-based TCOs ............... 26

2. $\mathrm{SnO}_{2}$-based TCOs $\ldots \ldots \ldots \ldots \ldots \ldots \ldots .27$

3. ZnO-based TCOs ................. 28

4. Other TCO systems ............... 29

D. Phase change materials .............. 29

VI. ENERGY-RELATED MATERIALS ......... 30

A. Thermoelectrics .................. 30

B. Lithium battery materials ............ 32

1. Cathode materials ............... 33

2. Anode materials ................. 35

C. Hydrogen storage materials ........... 36

1. Data mining and modeling ......... 36 
2. Thin film combinatorial work.........

3. Combinatorial studies of hydride

powders .......................

D. Catalysts for fuel cell anodes..........

VII. HIGH-THROUGHPUT MAPPING OF PHASE

DIAGRAMS .................

37

39

40

\section{INTRODUCTION AND HISTORICAL PERSPECTIVE}

High throughput (combinatorial) materials science is a relatively new paradigm for materials discovery and optimization that arose from the pharmaceutical industry ${ }^{1-4}$ and rapidly spread to other fields, several of which have matured and been the subject of review articles: organic coatings, ${ }^{5}$ sensor materials, ${ }^{6}$ inorganic materials, ${ }^{7}$ and functional materials. ${ }^{8-10}$ The idea of utilizing deliberately created composition gradients in co-deposited thin films to obtain significant portions of phase diagrams can be traced back to about fifty years ago for various ternary systems. ${ }^{11-14}$ In this review, we concentrate on applications of combinatorial materials science for electronic, magnetic, optical, and energy-related materials. As the emphasis of this review is on applications of these functional materials, ${ }^{15,16}$ library synthesis techniques, and properties measurement and analysis methods, are only covered as they apply to the materials of interest. Further, some high throughput fields of great importance, such as discovery and optimization of catalysts (well accepted and integrated into the field), are largely omitted from this review. In contrast to many of the topics covered in this review, highthroughput catalyst development is perhaps more prevalent in industry than in academia; ${ }^{17-21}$ a number of critical reviews and perspectives have been published, ${ }^{2,22-27}$ which we recommend to the reader. Another area where combinatorial strategies have played a significant role in advancing the field is in polymeric and soft materials, and recent reviews of this field are available. ${ }^{28,29}$

The combinatorial materials science approach ${ }^{24,30-33}$ is characterized by creation of a "library" sample that contains variations of the materials parameter of interest (typically composition, although this paper will discuss many others), and rapid, local, and automated measurements to interrogate the library for the property of interest, resulting in the generation of large data sets. These data sets are analyzed and generally displayed as a response surface (i.e., property as a function of variable of interest).

Combinatorial methodologies are generally faster and less expensive (likely true even taking initial equipment investments into account) than traditional (e.g., "one composition at a time") approaches. Further, since all experiments are carried out on the same library sample with the same measurement tool over a short time period, most systematic errors are eliminated, thus generating a comprehensive and reliable data set. Combinatorial materials science excels in the observation of trends and in screening, i.e., finding the "sweet spot" of the response surface, which enables rapid materials optimization.

In spite of the large number of papers published in the field of combinatorial materials science, industry has not adopted the new paradigm on a large scale, and there has been some skepticism. Some of this is due to the nature of the library films, which may contain local variations such as microstructural changes and stress variations that result directly from compositional variations; thus, the libraries are perceived as flawed, in that although only one variable is intentionally varied, other properties may change unintentionally, resulting in a perceived "unscientific" approach. Other reasons for skepticism are based on practicality, such as the high cost of a tool set for synthesizing library samples, the paucity of commercially available high throughput measurement instruments, and the highly skilled labor needed to run a combinatorial laboratory. While it is difficult to fully defend the notion that the composition of a library film can be varied while maintaining all other properties constant, the use of a greater number of analysis techniques should allow these complicated interactions to be sorted out. ${ }^{31}$

Another source of criticism is the frequent necessity to process the combinatorial library samples using different growth and post-growth processing techniques than will be employed in the production of the material for its intended application. This criticism can be mitigated to some extent by realizing that in combinatorial analysis, the "slope" of the property with regard to the parameter variation may be more important than the actual value of the property at any given value of the parameter. Also, combinatorial methodologies may not be useful for determination of properties that are highly structure sensitive. In any case, in an ideal combinatorial experiment, the results should be benchmarked to some key data that have been determined by more traditional means; in this review, we will endeavor to supply such information whenever possible.

Combinatorial materials science has traditionally been focused on materials discovery, screening, and optimization to combat the extremely high cost and long development time of new materials and their introduction into commerce. Going forward this new paradigm may be driven by other needs such as materials substitution and experimental verification of materials properties predicted by modeling and simulation.

\section{ELECTRONIC MATERIALS}

\section{A. Silicon microelectronics}

\section{Advanced gate stack materials}

Continuous scaling of complementary-metal-oxidesemiconductor (CMOS) transistors to meet the evolving requirements of the International Technology Roadmap for Semiconductors (ITRS) has made the traditional gate stack, $\mathrm{SiO}_{2}$ gate dielectric and polycrystalline $\mathrm{Si}$ (poly-Si) gate electrode, unsuitable for future integrated circuit devices. ${ }^{34}$ Further, as is shown in Fig. 1, only about a dozen elements were involved in integrated circuit manufacturing in 1990, as opposed to over fifty by the year 2000. The introduction of so many new elements and materials in the form of their alloys and compounds should have provided an excellent opportunity for the widespread use of combinatorial methodologies in the microelectronics industry. However, the 


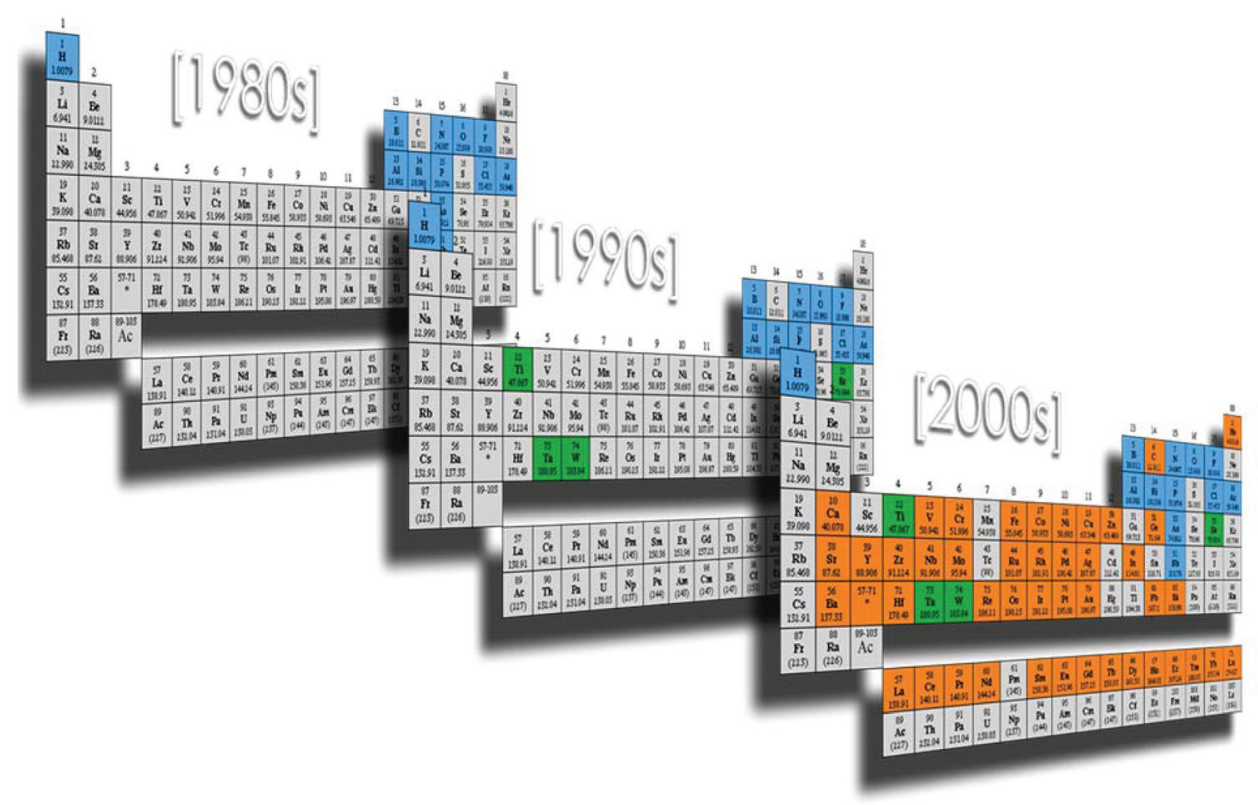

FIG. 1. Increasing utilization of different elements in the silicon microelectronics industry, as a function of time. Reproduced with permission from IBM Corporation.

industry was slow in adopting such high throughput techniques, perhaps due to the high cost of the suite of deposition and testing equipment required. ${ }^{35}$ Nonetheless, some excellent high throughput materials research has been focused on Si microelectronics.

a. High- $\kappa$ gate dielectrics. Since the advent of the integrated circuit, Fig. 2, $\mathrm{SiO}_{2}$ has been the gate dielectric material of choice for silicon microelectronics. ${ }^{36}$ However, since high gate capacitance is necessary to produce the required drive currents for integrated circuit devices, and further, capacitance is inversely proportional to gate dielectric thickness, $\mathrm{SiO}_{2}$ gate dielectrics have been scaled to ever thinner dimensions. Because $\mathrm{SiO}_{2}$ has a relatively low dielectric constant, $\kappa$, of 3.9, such scaling resulted in $\mathrm{SiO}_{2}$ layers so thin $(\sim 1.0 \mathrm{~nm})$ that excessive leakage current compromised their performance. ${ }^{37}$ Alternate, "high- $\kappa$ " gate dielectrics were therefore required. ${ }^{38}$

The first high throughput experiments in high dielectric constant electronic oxides (albeit for memory cells, not gate dielectrics) were by van Dover et al. ${ }^{39}$ Using multiple sputtering guns to create continuous composition spread (CCS) libraries, they found compositions in the $\mathrm{Zr}-\mathrm{Sn}-\mathrm{Ti}-\mathrm{O}$ system that displayed high figures of merit (based on the product of

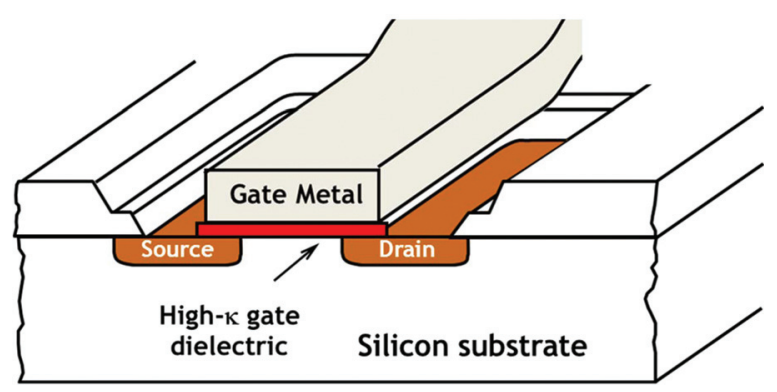

FIG. 2. Cross section of an integrated circuit transistor device, showing the gate stack (gate metal electrode plus gate dielectric), the source and drain regions, and the channel (in between the source and drain). the capacitance per unit area and the operating voltage), shown in Fig. 3. They studied about thirty different ternary oxide systems, sometimes making four thousand measurements per day on a single library.

High- $\kappa$ gate dielectric materials have been the subject of several combinatorial studies, starting with an investigation of the $\mathrm{HfO}_{2}-\mathrm{Y}_{2} \mathrm{O}_{3}-\mathrm{Al}_{2} \mathrm{O}_{3},{ }^{40-42}$ Fig. 4, and $\mathrm{ZrO}_{2}-\mathrm{Y}_{2} \mathrm{O}_{3}-\mathrm{Al}_{2} \mathrm{O}_{3}$, Fig. 5, systems. ${ }^{40}$ Pulsed laser deposition (PLD) or ion beam sputtering was used to deposit these CCS library films, Fig. 6, and a scanning microwave microscope, ${ }^{4,44}$ Fig. 5, was used to measure the dielectric properties of the films without having to fabricate capacitors. In addition, the use of $\mathrm{X}$-ray diffraction (XRD) analysis enabled Chikyow et al. ${ }^{40}$ to identify an amorphous $\mathrm{HfO}_{2}-\mathrm{Y}_{2} \mathrm{O}_{3}-\mathrm{Al}_{2} \mathrm{O}_{3}$ composition, having the ratio $6: 1: 3$, as the most promising gate dielectric candidate. Since the dielectric constant of $\mathrm{HfO}_{2}(\sim 20)$ is the highest of the three components in their system, it is not surprising that the amorphous dielectric of choice is rich in that oxide. XRD evidence ${ }^{41}$ indicated the presence of cubic, monoclinic, and amorphous phases in the $\mathrm{HfO}_{2}-\mathrm{Y}_{2} \mathrm{O}_{3}-\mathrm{Al}_{2} \mathrm{O}_{3}$ system. Non-combinatorial studies of $\mathrm{HfO}_{2}$ (Ref. 45) and the $\mathrm{HfO}_{2}-\mathrm{Al}_{2} \mathrm{O}_{3}$ (Ref. 46) system are in agreement with the basic finding of cubic and monoclinic phases, and the fact that $\mathrm{Al}_{2} \mathrm{O}_{3}$ addition stabilizes the amorphous phase. Whereas in the combinatorial study a $\mathrm{HfO}_{2}$ to $\left(\mathrm{Y}_{2} \mathrm{O}_{3}+\mathrm{Al}_{2} \mathrm{O}_{3}\right)$ ratio of 6:4 was sufficient to stabilize the amorphous phase after a $700{ }^{\circ} \mathrm{C}$ anneal for $30 \mathrm{~min}$, single composition $\mathrm{HfO}_{2}-\mathrm{Al}_{2} \mathrm{O}_{3}$ films with a $\mathrm{HfO}_{2}$ to $\mathrm{Al}_{2} \mathrm{O}_{3}$ ratio of $1.5: 1$ was stable in the amorphous phase up to $900{ }^{\circ} \mathrm{C}$, and films with a $\mathrm{HfO}_{2}$ to $\mathrm{Al}_{2} \mathrm{O}_{3}$ ratio of $1: 1.5$ remained amorphous after a $1050{ }^{\circ} \mathrm{C}$ rapid thermal anneal. ${ }^{46}$ This result highlights the importance of benchmarking combinatorial experiments with conventional experimental approaches.

Another recent high throughput study looked at the $\mathrm{RE}_{2} \mathrm{O}_{3}-\mathrm{Al}_{2} \mathrm{O}_{3}-\mathrm{HfO}_{2}$ systems, where $\mathrm{RE}$ is the rare earth elements, in this case $\mathrm{La}, \mathrm{Ce}$, and $\mathrm{Y}^{47}$ The goal of the experiment was to determine which compositions in these systems might grow epitaxially on Si (100) surfaces, and thus perhaps 

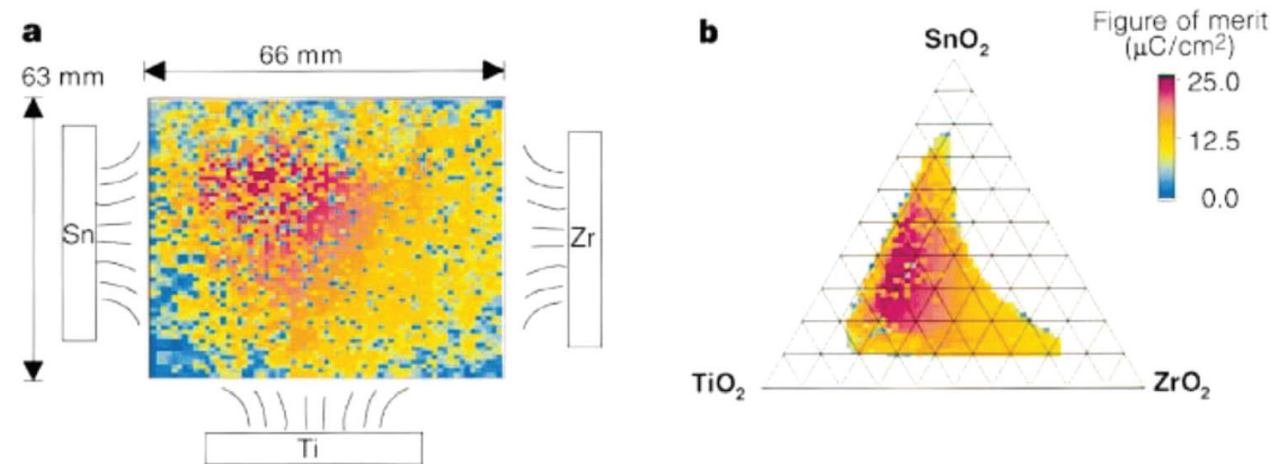

FIG. 3. Figure of merit (FOM) of $\mathrm{Zr}_{\mathrm{x}} \mathrm{Sn}_{\mathrm{y}} \mathrm{Ti}_{\mathrm{z}} \mathrm{O}_{2-\delta}$ dielectric thin films: (a) $\mathrm{FOM}$ as a function of position on the $6.2 \times 6.5 \mathrm{~cm}$ substrate, obtained by multiplying the capacitance and voltage measured at each point, and normalizing to the capacitor area. The location of the $\mathrm{Zr}$, Ti, and Sn guns is indicated schematically with respect to the position of the substrate; (b) same data mapped onto a conventional ternary composition diagram. Reprinted by permission from R. B. van Dover et al., Nature 392, 162 (1998). Copyright 1998 Macmillan Publishers Ltd.

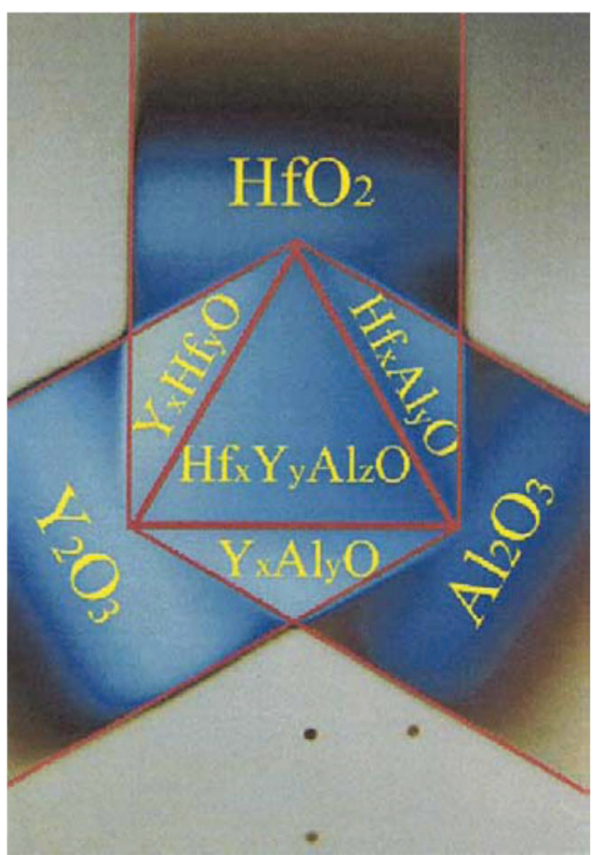

FIG. 4. Ternary composition spread film grown on a $\mathrm{Si}(100)$ substrate. The equilateral triangle in the center corresponds to the ternary phase diagram and the three isosceles regions around it correspond to three binary phase diagrams. Reproduced with permission from K. Hasegawa et al., Appl. Surf. Sci. 223, 229 (2004). Copyright 2004 Elsevier BV. mimic the low defect density, continuous random network structure of the native $\mathrm{SiO}_{2} / \mathrm{Si}$ interface. They discovered a new ionic structure that accommodated local atomic alterations as easily as native $\mathrm{SiO}_{2}$ and could serve as a model for advanced high- $\kappa$ gate dielectrics. One desirable composition, although exhibiting high leakage current most likely due to its small bad gap offset, was a $22 \mathrm{~nm}$ film of the composition $\mathrm{Ce}_{6}\left(\mathrm{AlO}_{3}\right)_{5.1}\left(\mathrm{SiO}_{3.5}\right)_{0.9}$. In the course of performing this work, the authors introduced a method for high throughput structural characterization of CCS libraries using high energy (synchrotron) $\mathrm{X}$-ray beams in transmission geometry. ${ }^{48}$ Using $100 \mathrm{~nm}$ thick films in the $\mathrm{Ce}_{2} \mathrm{O}_{3}-\mathrm{Al}_{2} \mathrm{O}_{3}-\mathrm{HfO}_{2}$ system, they were able to obtain texture, epitaxial relationship, and phase information in their combinatorial library film, Fig. 7. They were able to identify fifteen different phases because the films were relatively thick and they used an intense (synchrotron) beam. In general, laboratory XRD analyses of such combinatorial library films result in the identification of only the most predominant phases, such as has been observed for the TaN-AlN (gate metal electrode) system. $^{49}$

PLD has been the film growth technique of choice for several gate dielectric combinatorial experiments. Although PLD is not a commercially viable deposition technique because the process often involves the creation of myriad particles, it is a very valuable laboratory technique since it
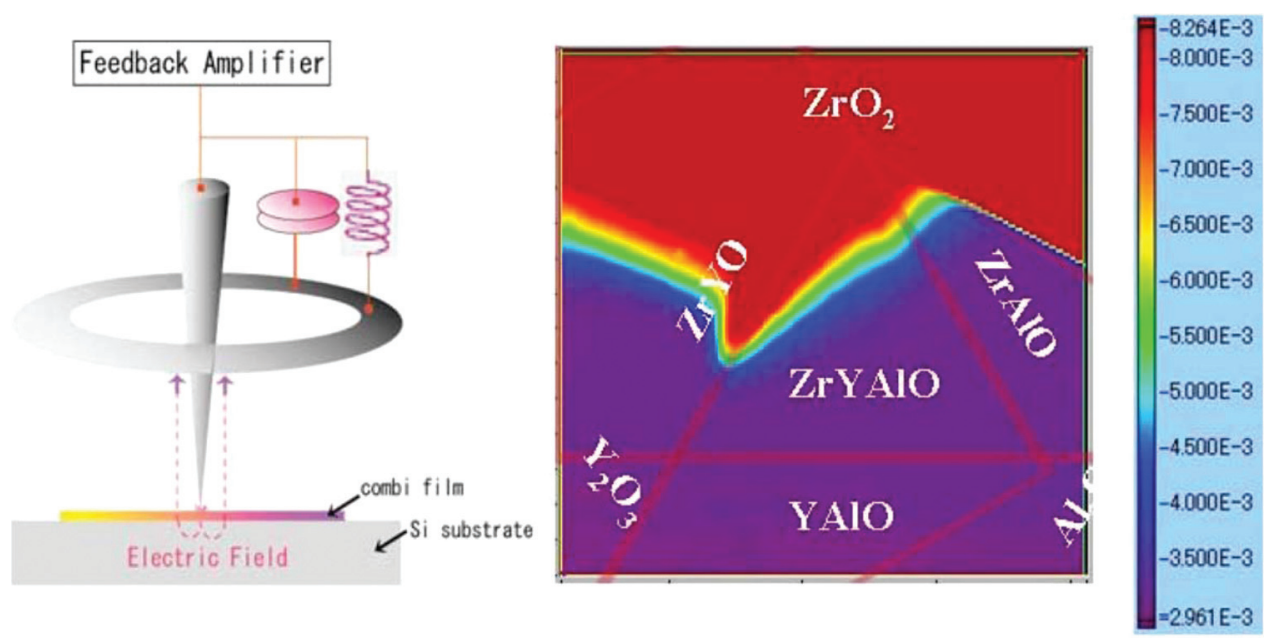

FIG. 5. Schematic illustration of the microwave microscope for dielectric property mapping, and a measured example for a $\left(\mathrm{ZrO}_{2}\right)_{\mathrm{x}}\left(\mathrm{Y}_{2} \mathrm{O}_{3}\right)_{\mathrm{y}}\left(\mathrm{Al}_{2} \mathrm{O}_{3}\right)_{\mathrm{z}}$ combinatorial library sample. Reproduced with permission from T. Chikyow et al., IEEE Cat. No. 06EX1294 (2006). Copyright 2006 IEEE. 


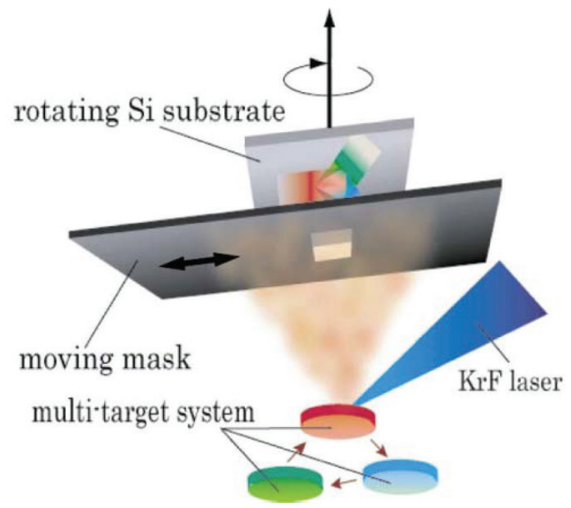

(a)
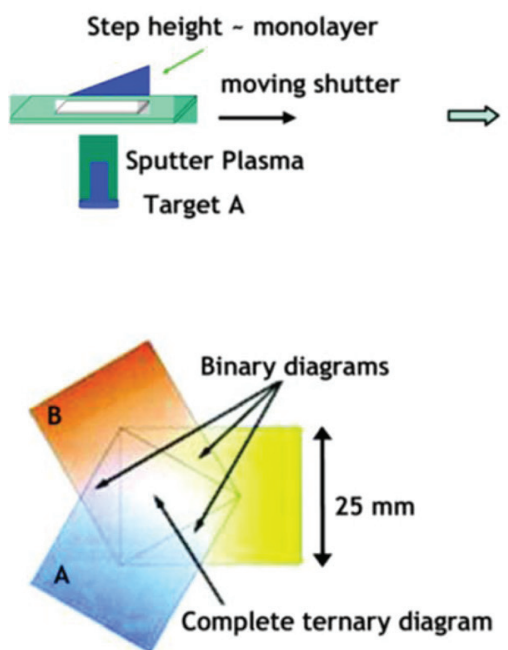

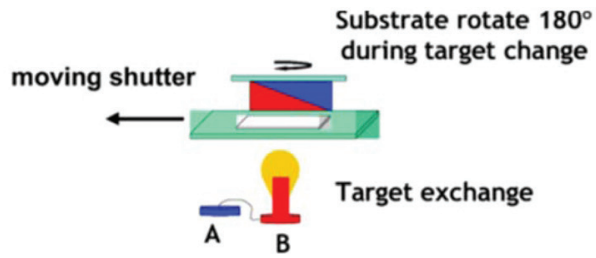

$\mathfrak{l}$

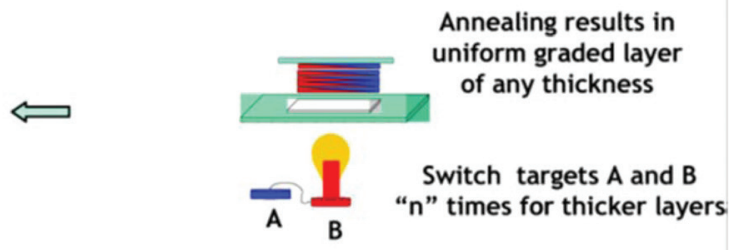

FIG. 6. (a) Schematic illustration of the ternary and binary composition spread method with sample rotation and moving mask system, first employed by Fukumura et al. ${ }^{104}$ Here, the PLD deposition method is shown as an example, but ion beam sputtering can also be used. Reproduced with permission from T. Chikyow et al., IEEE Cat. No. 06EX1294 (2006). Copyright 2006 IEEE. (b) SCHEMATIC showing the synthesis of a combinatorial "library" of the binary system A-B. A thin wedge (wedge height $\sim 0.5 \mathrm{~nm}$ ) of A is deposited, and the substrate is rotated $180^{\circ}$. A complementary wedge of $\mathrm{B}$ is then deposited. This is repeated " $\mathrm{N}$ " times, until the desired film thickness is achieved. If the substrate is rotated $120^{\circ}$ instead, a ternary (shown from above in the lower left), rather than a binary alloy system may be synthesized. Reproduced with permission from M. L. Green et al., Microelectron. Eng. 84, 2209 (2007). Copyright 2007 Elsevier B.

(b)

results in nearly perfect stoichiometry transfer between the target(s) and the deposited film. Recent studies were aimed at optimizing the compositional coverage of PLD films while maintaining a uniform thickness across the substrate. ${ }^{50,51}$ In these studies, $\mathrm{Al}_{2} \mathrm{O}_{3}-\mathrm{HfO}_{2}-\mathrm{Y}_{2} \mathrm{O}_{3}$ combinatorial libraries were deposited by the "natural" CCS technique, i.e., films were sequentially deposited at various $(\mathrm{x}, \mathrm{y})$ locations on the substrate wafer, from different targets; no shutters or masks were used, unlike the case illustrated in Fig. 6. The composition and thickness of such films can be tuned by adjusting the laser spot size and fluence, background gas pressure, and target geometry. Using a simple bimodal cosine power distribution function for the deposition rate from the three individual targets, the composition and thickness of ternary library films could be predicted. The experimentally determined film thickness (by spectroscopic reflectometry) and composition (by electron probe microanalysis wavelength dispersive spectroscopy (EPMAWDS) results agreed with the predictions to within measurement resolution, as is shown in Figs. 8 and 9. This modeling approach results in a high degree of confidence that one can predict thickness and compositional coverage, making the combinatorial PLD tool very useful for routine library design, while avoiding costly and time consuming experimental verification of library composition.
In another PLD study of gate dielectrics, ${ }^{52}$ combinatorial library films of the $\mathrm{HfO}_{2}-\mathrm{TiO}_{2}-\mathrm{Y}_{2} \mathrm{O}_{3}$ system were grown. $\mathrm{TiO}_{2}$ has a relatively high dielectric constant, $\kappa \sim 80$, but is not thermodynamically stable on $\mathrm{Si}^{53}$ Thus, the goal of this experiment was to screen for a gate dielectric composition that had a higher dielectric constant than that of $\mathrm{HfO}_{2}$ (about 20), was thermally stable on $\mathrm{Si}$, and also be stable as an amorphous phase. Interestingly, it was found that films in the $\mathrm{HfO}_{2}-\mathrm{TiO}_{2}-\mathrm{Y}_{2} \mathrm{O}_{3}$ library exhibited a visible boundary separating amorphous and crystalline phases, Fig. 10, and that this boundary could be manipulated by varying substrate temperature or $\mathrm{TiO}_{2}$ distribution. Further, the authors were able to eliminate the visual boundary, and therefore realize two microstructural extremes; the entire $\mathrm{HfO}_{2}-\mathrm{TiO}_{2}-\mathrm{Y}_{2} \mathrm{O}_{3}$ ternary film could be amorphous after deposition at $300^{\circ} \mathrm{C}$, or crystalline in the $\mathrm{HfO}_{2}-\mathrm{Y}_{2} \mathrm{O}_{3}$ binary system. Electrical measurements demonstrated, Fig. 11, that the dielectric constant of the library films could be varied between about 10 and 120. Surprisingly, some of the highest dielectric constants are observed near the $\mathrm{TiO}_{2}-\mathrm{Y}_{2} \mathrm{O}_{3}$ binary system, ${ }^{54}$ containing only small fractions of $\mathrm{HfO}_{2}$.

Another recent study focused on the $\mathrm{Y}_{2} \mathrm{O}_{3}-\mathrm{Al}_{2} \mathrm{O}_{3}$ system, with the goal of forming high dielectric constant, thermally stable, yttrium aluminate gate dielectrics. ${ }^{55}$ The library films were grown by PLD using discrete masks, and 

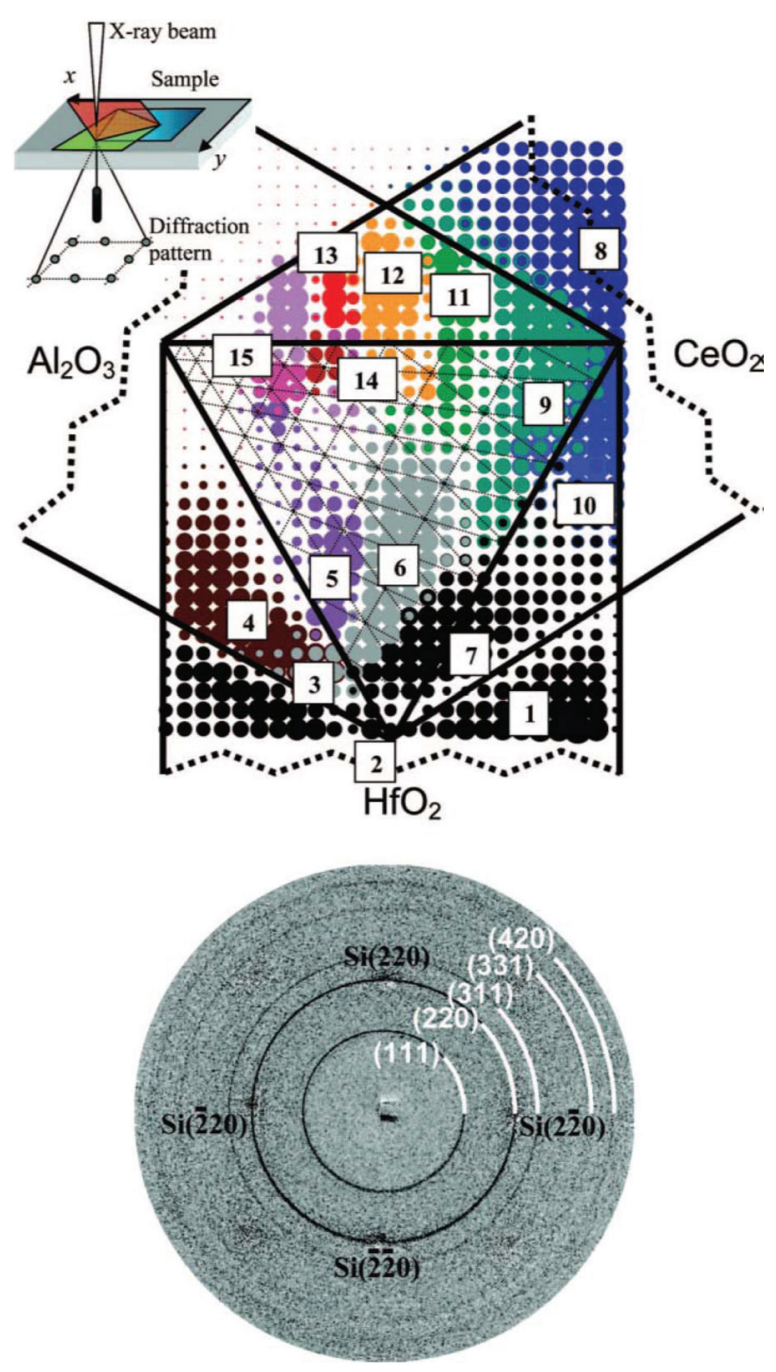

FIG. 7. Bubble plot reconstruction of the crystal phase diagram for the $\mathrm{CeO}_{2}-\mathrm{Al}_{2} \mathrm{O}_{3}-\mathrm{HfO}_{2} \mathrm{CCS}$ thin film on a $\mathrm{Si}(001)$ substrate. The various phases are numbered. The lower figure is an HEXRD diffraction image taken from the polycrystalline $\mathrm{CeO}_{2}$ part of the composition spread. Reprinted with permission from D. A. Kukuruznyak et al., Appl. Phys. Lett. 91, 071916 (2007). Copyright 2007 American Institute of Physics.

the effects of $\mathrm{Al}$ doping and annealing on the thermal stability of the $\mathrm{Y}_{2} \mathrm{O}_{3} / \mathrm{Si}$ gate stack were studied by X-ray photoemission spectroscopy (XPS) and X-ray absorption spectroscopy. Although Al doping was effective in increasing the band gap of $\mathrm{Y}_{2} \mathrm{O}_{3}$, Fig. 12, it did not suppress yttrium silicate formation via diffusion of $\mathrm{SiO}_{2}$ from the interfacial layer into the yttrium aluminate; annealing promoted the formation of silicate compounds in the film via mixing of the silicate layer and the $\mathrm{Si}$ substrate. The observed band gap bowing in the $\mathrm{Y}_{2} \mathrm{O}_{3}-\mathrm{Al}_{2} \mathrm{O}_{3}$ system was attributed to parabolic changes in the conduction band offset as a function of $\mathrm{Al}$ concentration.

The development of high-throughput chemical vapor deposition (CVD) methodologies for high- $\kappa$ gate dielectrics ${ }^{56-58}$ is an important effort, since CVD and a related technology, atomic layer deposition (ALD) ${ }^{59}$ may be the manufacturing methods of choice for these critical films. Thus, it is important to explore the feasibility of performing combinatorial experimentation in such a platform. While it is non-trivial to generate large composition variation in a CVD chamber, they can be achieved by controlling the flow of precursor gases. ${ }^{60,61}$ Equally important to achieving compositional variations on the wafers is the need to achieve process parameter (i.e., pressure and flow) variations.

b. Metal gate electrodes. Substitutions for the poly-Si gate electrode, the second component of the CMOS gate stack of Fig. 2, using true metals or metalloids that would provide higher carrier densities and therefore lower resistivities as well as less carrier depletion, were not developed until after the high- $\kappa$ gate dielectrics. Therefore, the selection of metal gate substitutes for poly-Si, which must be compatible with the new high- $\kappa$ gate dielectrics, is not as advanced. For high performance CMOS applications, the metal gate electrodes must have work functions $\left(\Phi_{\mathrm{m}}\right)$ that are aligned with the conduction $(4.05 \pm 0.2 \mathrm{eV})$ and valence $(5.17 \pm 0.2 \mathrm{eV})$ bands of $\mathrm{Si}$ for $\mathrm{n}$-channel metal-oxide-semiconductor (NMOS) and p-channel metal-oxide-semiconductor (PMOS) applications, respectively. ${ }^{34}$

In one of the first high throughput studies of metal gate electrodes, Ni-Ti-Pt ternary libraries were deposited by magnetron sputtering through moving masks on $\mathrm{HfO}_{2}$ dielectrics, and studied systematically by WDS, micro-XRD, and capacitance-voltage (C-V) and current-voltage (I-V) analyses. ${ }^{62}$ WDS results showed that compositional coverages up to about $90 \%$ for $\mathrm{Ni}$ and $\mathrm{Ti}$, and $75 \%$ for $\mathrm{Pt}$ were achieved in the library film, as can be seen in Fig. 13. A more negative flatband voltage $\left(\mathrm{V}_{\mathrm{fb}}\right)$ was observed close to the Ti-rich corner than to the Ni- and Pt-rich corners, implying a smaller $\Phi_{\mathrm{m}}$ near the Ti-rich corner, and higher $\Phi_{\mathrm{m}}$ near the Ni- and Pt-rich corners. Not only is that observed, as can be seen in Fig. 13(b), but also the $\Phi_{\mathrm{m}}$ values measured for the nearly pure metals (i.e., near the corners) in this combinatorial experiment are in very good agreement with bulk literature values for $\mathrm{Ni}$, Ti, and Pt. Thus, combinatorial methodologies proved to be accurate and useful in surveying the large compositional space of ternary alloy metal gate electrode systems.

When using a moving shutter for library film deposition $^{63-65}$ to deposit a wedge of material at a time, as shown in Fig. 6, the shape of the wedge, i.e., amount deposited as a function of distance in the plane of the wafer, must be calibrated very carefully if one is to have a sufficiently accurate idea of the compositional spread. The wedge height is very often small (a few tenths of a nm) to enable, even in the as-deposited condition, atomic mixing in the direction perpendicular to film growth. Figure 14 illustrates the use of Rutherford backscattering (RBS) as a calibration procedure for a wedge of TaN deposited by sputtering with the use of a moving shutter. ${ }^{49,65}$ RBS is of course very helpful for calibrating deposition profiles using any deposition method.

High throughput experiments were also performed on the Mo- $\mathrm{Ru}^{66}$ and Pt- $\mathrm{W}^{67}$ metal gate electrode systems deposited on high- $\kappa$ dielectrics. As can be seen in Fig. 15, the addition of 10\%-30\% Mo in Ru electrodes, deposited on Hf-Si$\mathrm{O}-\mathrm{N}$, enables tuning of the $\mathrm{V}_{\mathrm{fb}}$ values, likely due to Mo segregation at the metal gate/high- $\kappa$ interface. Further, carbon incorporation was found to be effective in refining the metal 

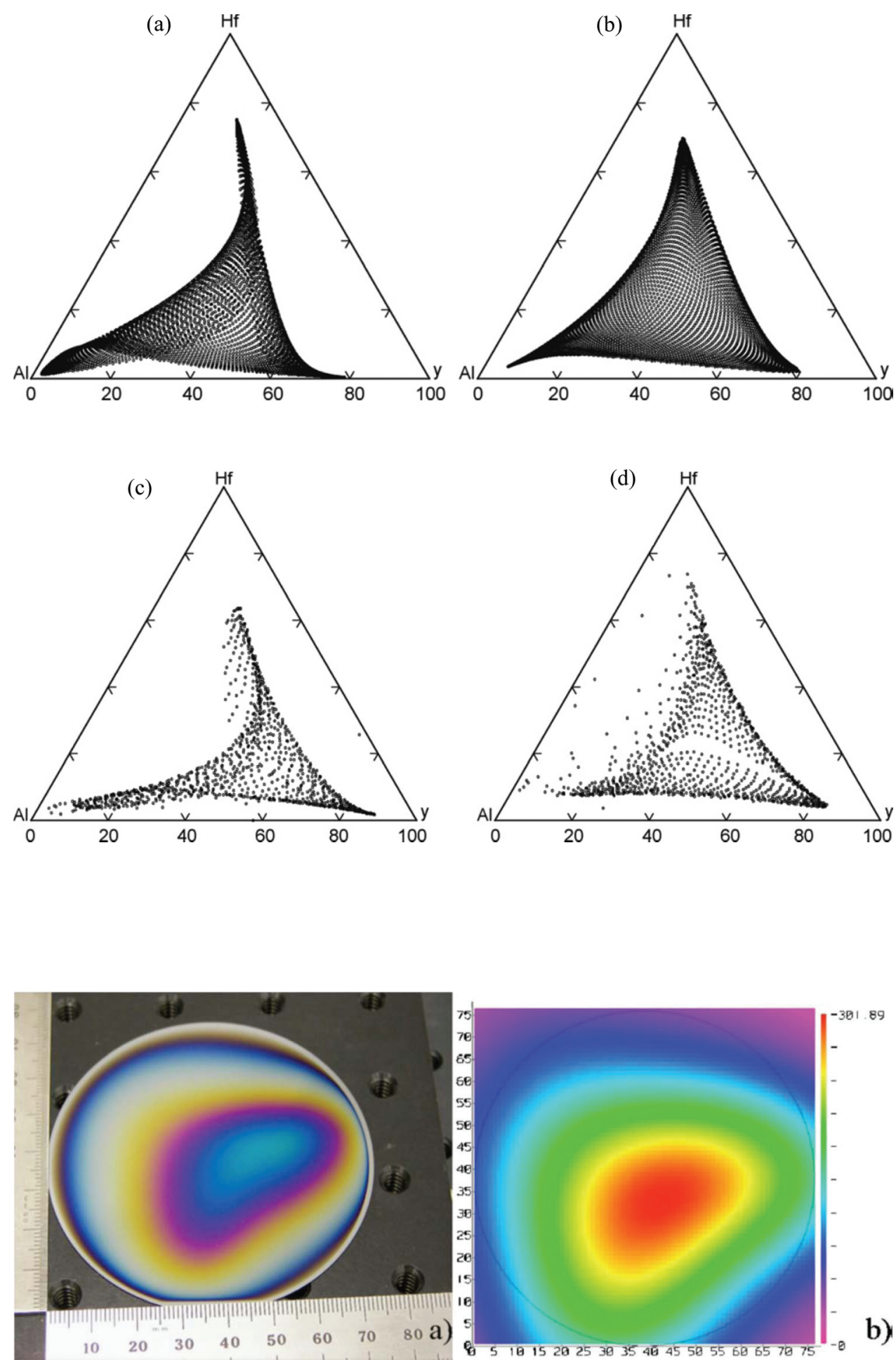

b)
FIG. 8. Comparison of simulated ((9a) and (9b)) and experimentally determined $((9 \mathrm{c})$ and $(9 \mathrm{~d}))$ composition maps for PLD libraries in the Hf-Al- $\mathrm{YO}_{\mathrm{x}}$ system. The library in (9a) was deposited at $\mathrm{P}_{\mathrm{O} 2}=1.33 \mathrm{~Pa}$, and the library in $(9 \mathrm{~b})$ at $\mathrm{P}_{\mathrm{O} 2}=13.3 \mathrm{~Pa}$. Reproduced with permission from P. K. Schenck et al., Appl. Surf. Sci. 254, 781 (2007). Copyright 2004 Elsevier BV.
FIG. 9. (a) Photograph and (b) predicted thickness map for a PLD Hf-Al-YO library film deposited with $\mathrm{P}_{\mathrm{O} 2}=13.3 \mathrm{~Pa}$. Reprinted with permission from N. D. Bassim et al., Rev. Sci. Instrum. 78, 072203 (2007). Copyright 2008 American Institute of Physics.

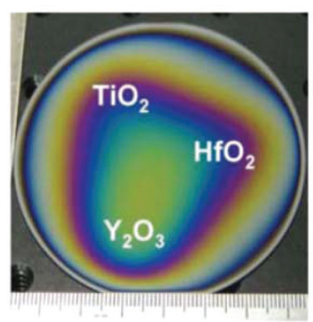

$300^{\circ} \mathrm{C}$

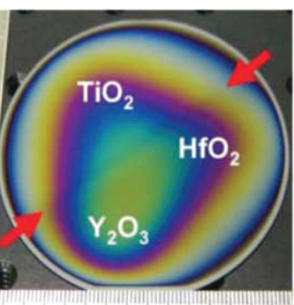

$400^{\circ} \mathrm{C}$

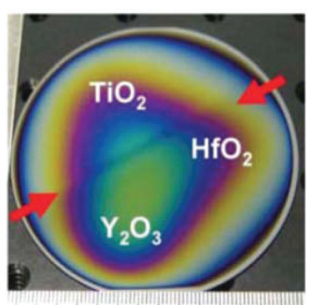

$500^{\circ} \mathrm{C}$

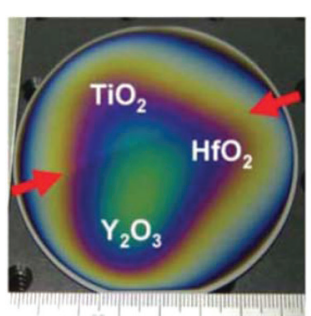

$600^{\circ} \mathrm{C}$
FIG. 10. Photographs of four $\mathrm{HfO}_{2}-$ $\mathrm{TiO}_{2}-\mathrm{Y}_{2} \mathrm{O}_{3}$ library films, each deposited at a different temperature. Those library films deposited at $\mathrm{T} \geq 400{ }^{\circ} \mathrm{C}$ show a clear boundary separating the $\mathrm{TiO}_{2}$-rich region from the $\mathrm{HfO}_{2}-\mathrm{Y}_{2} \mathrm{O}_{3}$ region. Reprinted with permission from J. L. Klamo et al., J. Appl. Phys. 107, 054101 (2010). Copyright 2008 American Institute of Physics. 


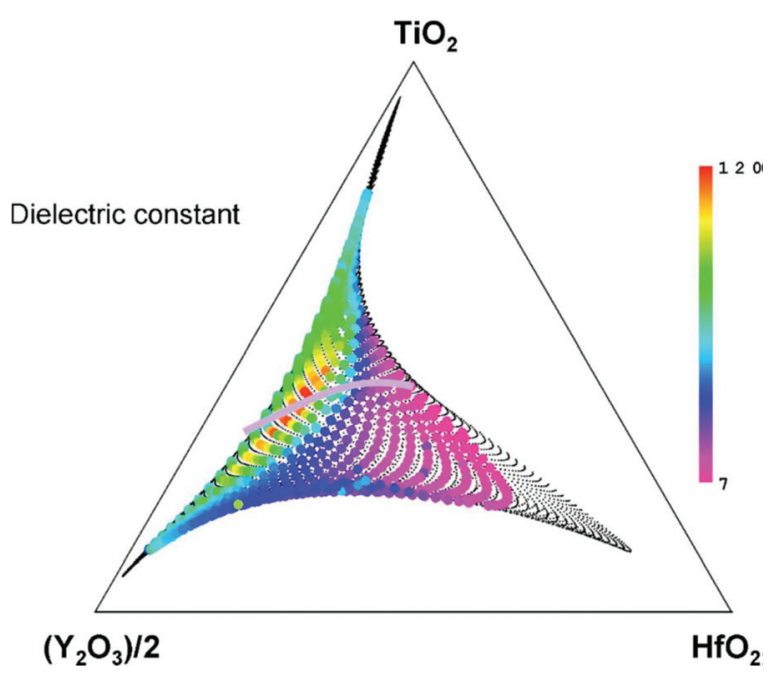

FIG. 11. Ternary diagram showing the predicted compositional coverage (small points) of the $500{ }^{\circ} \mathrm{C}_{\mathrm{HfO}_{2}}-\mathrm{TiO}_{2}-\mathrm{Y}_{2} \mathrm{O}_{3}$ library film, and the measured relative dielectric constant (filled colored points) extracted from the $\mathrm{C}-\mathrm{V}$ characteristics of the as-deposited film. The overlaid line represents the approximate location of the crystalline-amorphous boundary. Reprinted with permission from J. L. Klamo et al., J. Appl. Phys. 107, 054101 (2010). Copyright 2010 American Institute of Physics.

gate electrode grain size, thereby adding another $\mathrm{V}_{\mathrm{fb}}$ control mechanism. Similarly, a wide control range $(\sim 1.2 \mathrm{~V})$ of $\mathrm{V}_{\mathrm{fb}}$ was observed for $(\mathrm{Pt}-\mathrm{W}) / \mathrm{La}_{2} \mathrm{O}_{3}$ gate stacks. Again, the effect was attributed to charge transfer at the metal gate $/ \mathrm{La}_{2} \mathrm{O}_{3}$ interface, which induced an increase in the effective work function, especially at high $\mathrm{Pt}$ composition ratios, as can be seen in Fig. 16. The control effect was much stronger for $\mathrm{La}_{2} \mathrm{O}_{3}$ than for $\mathrm{HfO}_{2}$ gate dielectrics.

Metalloids such as metal nitrides and carbides have attracted attention as potential gate metal electrodes due to their superior thermal and chemical resistance compared to metals. Among the many metal gate candidates, TaN has shown very useful $\Phi_{\mathrm{m}}$ tunability through alloying with various elements. ${ }^{68-71}$ In particular, $\mathrm{Ta}_{1-\mathrm{x}} \mathrm{Al}_{\mathrm{x}} \mathrm{N}_{\mathrm{y}}$ alloys are easily deposited, with very good electrical and chemical properties. ${ }^{70}$ The $\mathrm{Ta}_{1-\mathrm{x}} \mathrm{Al}_{\mathrm{x}} \mathrm{N}_{\mathrm{y}}$ system is an ideal combinatorial materials science problem because systematic measurement of $\Phi_{\mathrm{m}}$ across the wide composition range, $\mathrm{x}$, is not trivial, since capacitor fabrication and characterization based on a
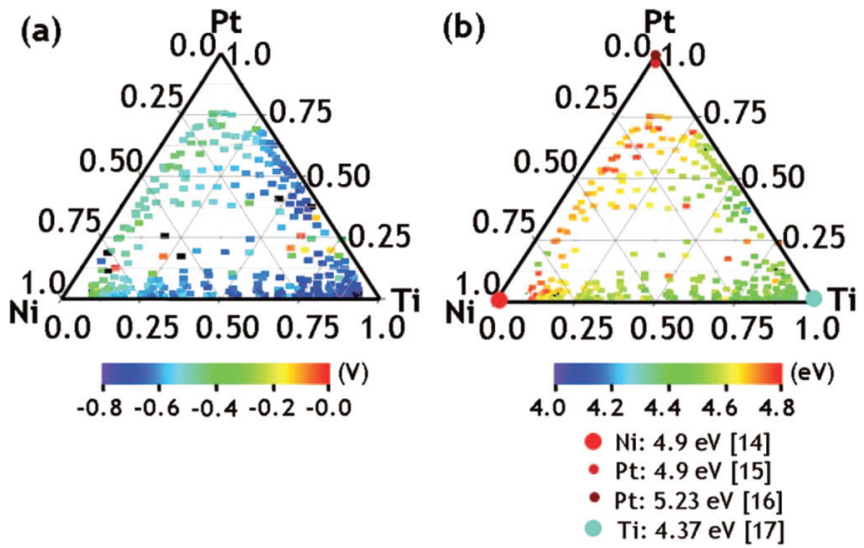

FIG. 13. (a) Map of the flatband voltage shift extracted from the C-V characteristics at $1 \mathrm{kHz}$ across the Ni-Ti-Pt library, and (b) map of the extracted work functions across the library. Round symbols in the three corners denote literature values. Reprinted with permission from K. S. Chang et al., Appl. Phys. Lett. 89, 142108 (2006). Copyright 2008 American Institute of Physics.

"one-composition-at-a-time" approach are extremely time consuming; thus very little data are available for this metal gate alloy system. ${ }^{72}$ Recently, a combinatorial approach was applied to $\Phi_{\mathrm{m}}$ extraction for the metal gate electrode system $\mathrm{Ta}_{1-\mathrm{x}} \mathrm{Al}_{\mathrm{x}} \mathrm{N}_{\mathrm{y}}$ deposited on $\mathrm{HfO}_{2} \cdot{ }^{49}$ Over two thousand capacitors on four identical metal gate libraries (albeit with differing capacitance due to $\mathrm{SiO}_{2}$ underlayers of different thicknesses) were automatically measured, from which $V_{\mathrm{fb}}$ shifts were extracted and $\Phi_{\mathrm{m}}$ determined. Figure 17 shows the array of test capacitors in the library sample; although a composition range of $0.05 \leq \mathrm{x} \leq 0.85$ was achieved on the library, electrical measurements were only performed on samples with $0.05 \leq \mathrm{x} \leq 0.50$, because higher $\mathrm{Al}$ concentrations were not suitably conductive. As can be seen from Fig. $18, \Phi_{\mathrm{m}}$ of the $\mathrm{Ta}_{1-\mathrm{x}} \mathrm{Al}_{\mathrm{x}} \mathrm{N}_{\mathrm{y}} / \mathrm{HfO}_{2} / \mathrm{SiO}_{2} / \mathrm{Si}$ gate stack structures can be tuned as a function of gate metal composition over a wide composition range, as well as by annealing. From these results, it was suggested that $\mathrm{Ta}_{0.9} \mathrm{Al}_{0.1} \mathrm{~N}_{1.24}$ gate metal electrodes may be useful for PMOS applications. Figure 18 is a noteworthy example of the power of the combinatorial technique. In this graph, $\Phi_{\mathrm{m}}$ is shown as a function of gate metal composition, with about thirty compositions represented, as
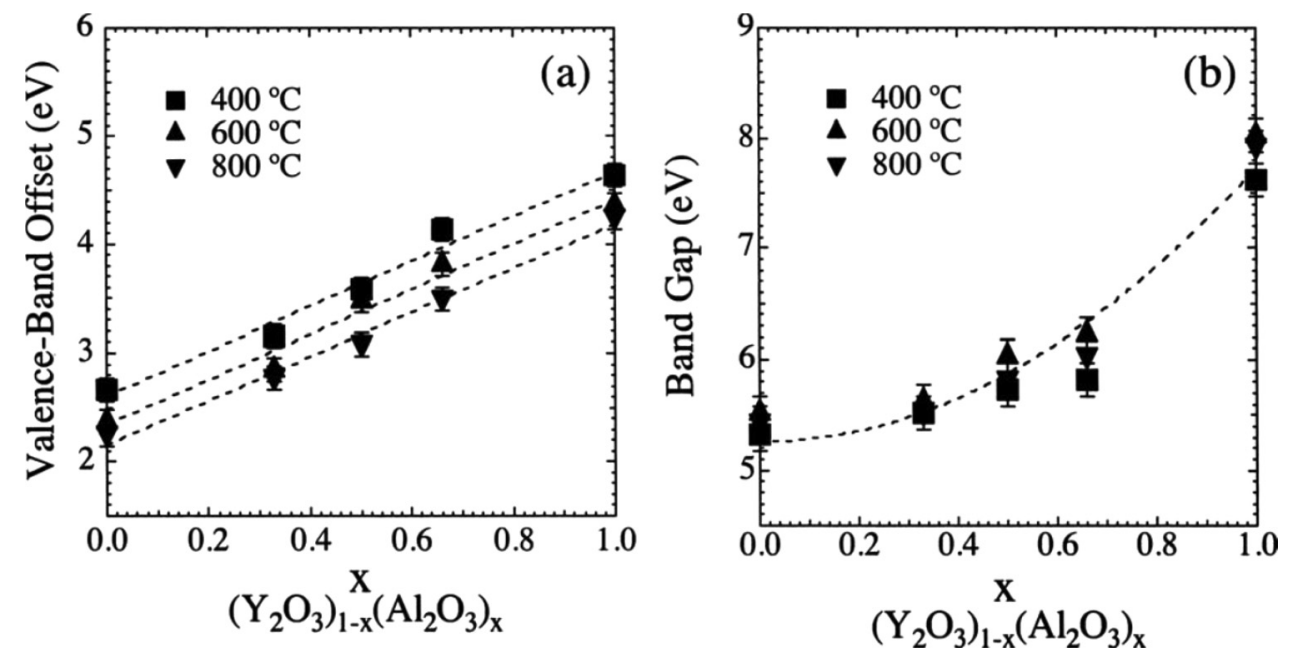

FIG. 12. Al concentration dependence of (a) valence-band offset and (b) band gap by annealing samples at 400,600 , and $800^{\circ} \mathrm{C}$. The dashed lines indicate (a) linear and (b) parabolic fitting results. Reprinted with permission from S. Toyoda et al., J. Vac. Sci. Technol. A 28(1), 16-19 (2010). Copyright 2010 American Institute of Physics. 


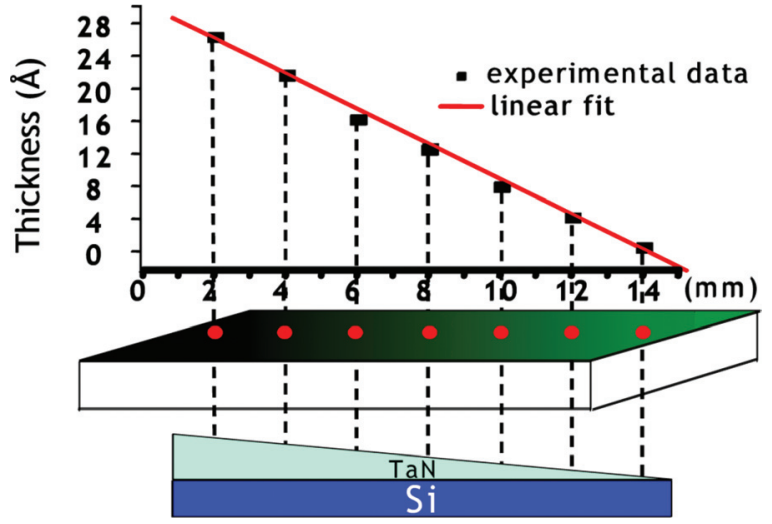

FIG. 14. Compositional calibration of a TaN-AlN library sample achieved by performing RBS analysis on a wedge profiled, moving shutter-deposited TaN (for example) film. Reproduced with permission from M. L. Green et al., Microelectron. Eng. 84, 2209 (2007). Copyright 2007 Elsevier BV.

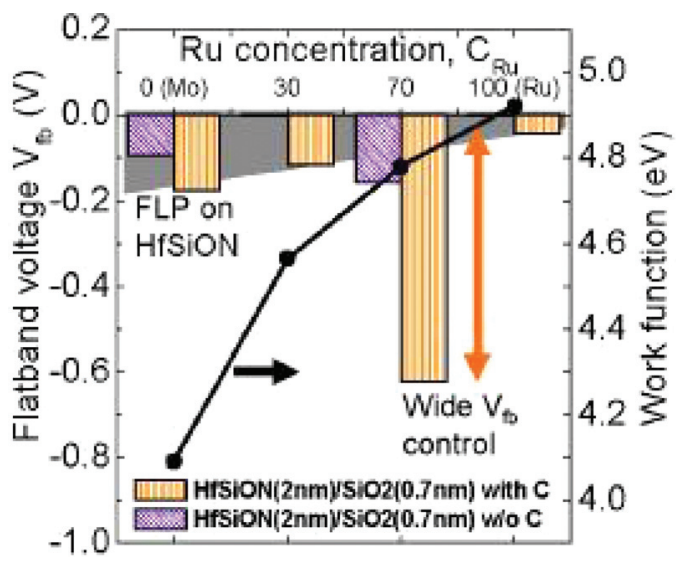

FIG. 15. At $\mathrm{C}_{\mathrm{Ru}}=0 \%, 30 \%$, and $100 \%$, the values in $\mathrm{V}_{\mathrm{fb}}$ are typical of metal/high- $\kappa$ interfaces, i.e., much narrower $\mathrm{V}_{\mathrm{fb}}$ controllability on high- $\kappa$ films than work function values. However, at $\mathrm{C}_{\mathrm{Ru}}=30 \%$, a wide controllability of $0.58 \mathrm{~V}$ has been achieved on $\mathrm{HfiSiON}$ after $500^{\circ} \mathrm{C}$ due to Mo segregation at the interface. Modification of the microstructure due to carbon incorporation plays a key role. Reproduced with permission from 2007 IEEE Electron Devices Meeting - IEDM 2007, 345. Copyright 2007 IEEE.

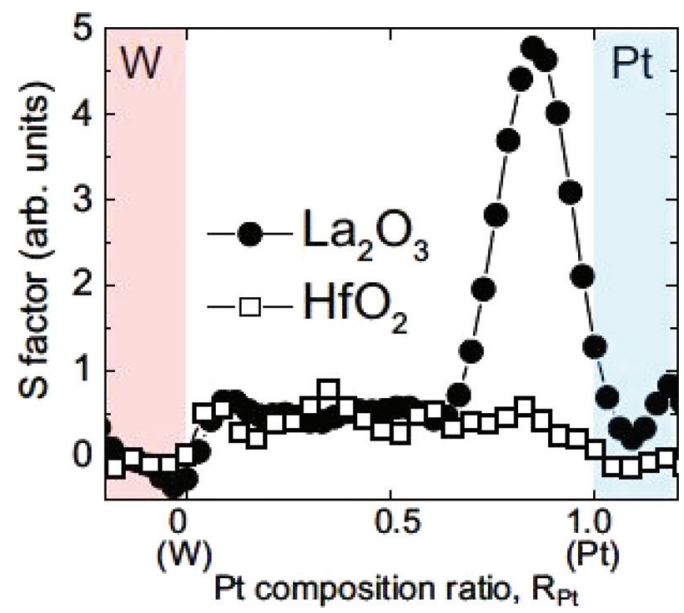

FIG. 16. Slope factor $\mathrm{S}$ (derivative of $\mathrm{V}_{\mathrm{fb}}$ as a function of work function), as a function of $\mathrm{Pt}-\mathrm{W}$ composition after a $450{ }^{\circ} \mathrm{C}$ forming gas anneal followed by a $350{ }^{\circ} \mathrm{C}$ oxidizing gas anneal. Note that the $\mathrm{S}$ factor of $\mathrm{La}_{2} \mathrm{O}_{3}$ overlaps that of $\mathrm{HfO}_{2}$ except for an anomalous increase in the range $0.7<\mathrm{Pt}<1$. Reproduced with permission from T. Chikyow et al., IEEE Cat. No. 06EX1294 (2006). Copyright 2006 IEEE. (a)

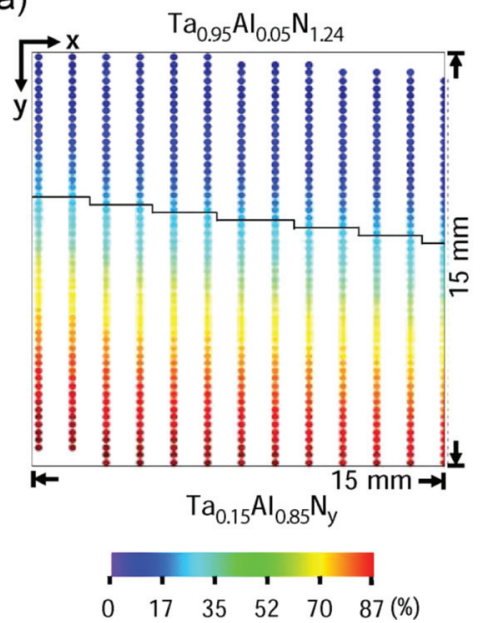

(b)

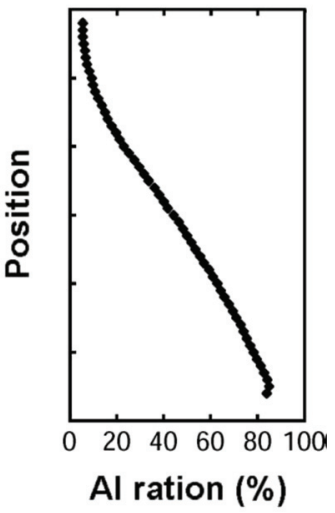

FIG. 17. (a) Composition map of the $\mathrm{Ta}_{1-x} \mathrm{Al}_{\mathrm{x}} \mathrm{N}_{\mathrm{y}}$ composition spread, as determined by WDS. The dimension of the library is $15 \mathrm{~mm} \times 15 \mathrm{~mm}$. Each dot represents a capacitor, and (b) plot of the composition variation across the sample. A wide composition range, $\mathrm{x}=0.05$ to 0.85 , was achieved. Reproduced with permission from K. S. Chang et al., IEEE Trans. Electron Devices 55, 2641 (2008). Copyright 2008 IEEE.

opposed to isolated data for only a few of those composition points found in the literature. Further, the data instantly reveal two other phenomena. First, one can see the consistent increase in $\Phi_{\mathrm{m}}$ after anneals at higher temperatures than the initial forming gas anneal $\left(500^{\circ} \mathrm{C}\right)$, and second, the thermal instability after the $1000{ }^{\circ} \mathrm{C}$ anneal of gate electrodes containing less than about $27 \% \mathrm{Al}$. Consistent and comprehensive sets of working data such in Fig. 18 are almost nonexistent in the semiconductor microelectronics literature.

Another metalloid system, Ta-C-N, has attracted attention as a potential metal gate electrode, also due to the possibility of $\Phi_{\mathrm{m}}$ tuning, high temperature, chemical stability, and good mechanical properties. Combinatorial Ta-C-N library films were deposited by magnetron sputtering on $\mathrm{HfO}_{2}{ }^{73}$ and WDS and fluorescence yield near edge spectroscopy (FYNES) were used to quantitatively determine the

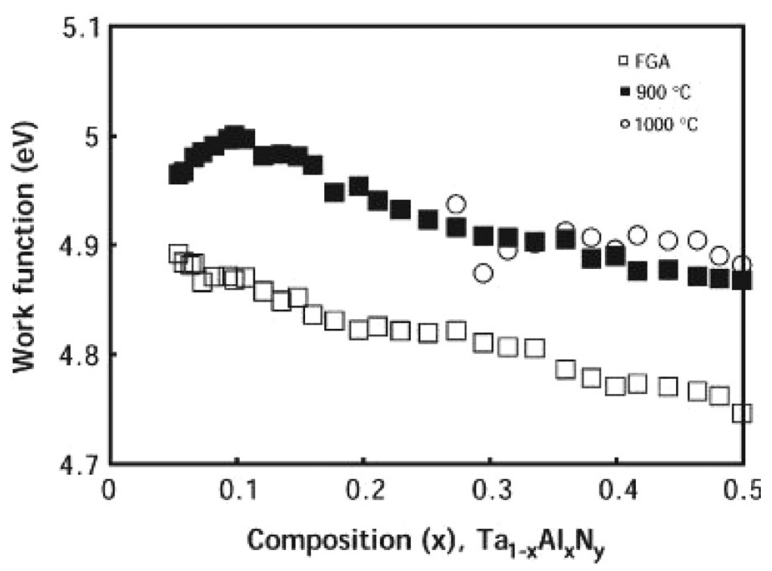

FIG. 18. Plot of the extracted work functions $(\Phi m)$ for the $\mathrm{Ta}_{1-\mathrm{x}} \mathrm{Al}_{\mathrm{x}} \mathrm{N}_{\mathrm{y}}$ composition spreads. $\Phi \mathrm{m}$ was extracted as a function of $\mathrm{Al}$ content after a forming gas anneal $\left(500^{\circ} \mathrm{C}\right)$, and $900^{\circ} \mathrm{C}$ and $1000^{\circ} \mathrm{C}$ rapid thermal anneals. After the $1000^{\circ} \mathrm{C}$ anneal, for $\mathrm{x}<0.27$, ,m could not be mapped due to degradation of the capacitors. Reproduced with permission from K. S. Chang et al., IEEE Trans. Electron Devices 55, 2641 (2008). Copyright 2008 IEEE. 
composition across these films. Interestingly, scanning micro-XRD determined that a solid solution of $\mathrm{Ta}(\mathrm{C}, \mathrm{N})_{\mathrm{x}}$ forms and extends to compositions that were previously unknown $(0.3 \mathrm{Ta} \leq 0.5$ and $0.57 \leq \mathrm{Ta} \leq 0.67)$, as is shown in Fig. 19. In addition, the thermal stability of the Ta-C-N/ $\mathrm{HfO}_{2}$ library film was studied using high resolution transmission electron microscopy (TEM), which showed that the $\mathrm{Ta}-\mathrm{C}-\mathrm{N} / \mathrm{HfO}_{2} / \mathrm{SiO}_{2} / \mathrm{Si}$ gate stack exhibited good thermal stability up to $950{ }^{\circ} \mathrm{C}$. ${ }^{74}$

\section{FERROELECTRIC, PIEZOELECTRIC, AND MULTIFERROIC MATERIALS}

Ferroelectrics, which exhibit a spontaneous electric polarization that can be reversed by the application of an external electric field, are an important class of functional materials, with a variety of applications. ${ }^{75,76}$ In thin film form, they are important for non-volatile memory devices. Recently, their applications for tunable microwave devices have been explored. Depending on the intended application, various properties such as dielectric constant and its tunability, dielectric loss, spontaneous polarization, coercive field, and breakdown voltage must be optimized. Many ferroelectric materials are also good piezoelectrics, which are ubiquitous in a wide range of sensor and actuator applications. ${ }^{77,78}$ One important research direction in the piezoelectrics community is the identification of lead-free, high piezoelectric coefficient replacements for the commonly used compound $\mathrm{Pb}(\mathrm{Zr}, \mathrm{Ti}) \mathrm{O}_{3}$ (PZT).

Ferroelectric materials often have a perovskite or related crystal structure. Thus, the emphasis of some of the first combinatorial studies was on synthesis of perovskite structures in thin film libraries with a large compositional variation. Chang et al. ${ }^{79}$ employed the precursor approach to fabricate $(\mathrm{Ba}, \mathrm{Sr}) \mathrm{TiO}_{3}$ thin films and systematically investigate the effect of transition metal (TM) dopants on their dielectric constants and losses. Different thicknesses of amorphous $\mathrm{BaF}_{2}, \mathrm{SrF}_{2}, \mathrm{TiO}_{2}$ layers were deposited to control the desired final A-site composition of the $(\mathrm{Ba}, \mathrm{Sr}) \mathrm{TiO}_{3}$ thin film at each site on the library. A thermal process involving a series of annealing steps to interdiffuse the precursor layers

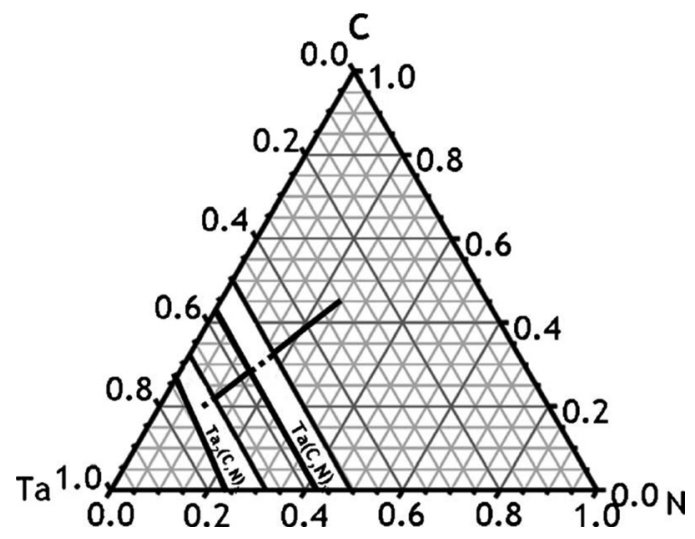

FIG. 19. Ta-C-N library film composition data plotted in ternary composition space (the solid and dashed black line). $\mathrm{Ta}(\mathrm{C}, \mathrm{N})_{\mathrm{x}}$ and $\mathrm{Ta}_{2}(\mathrm{C}, \mathrm{N})_{\mathrm{x}}$ equilibrium phase regions are also shown. Reprinted with permission from K. S. Chang et al., Appl. Phys. Lett. 96, 192114 (2010). Copyright 2010 American Institute of Physics. (and dopants), remove $\mathrm{F}_{2}$, and crystallize the titanate compounds was developed. Cross-sectional TEM revealed that quasi-epitaxial single crystalline thin films can be fabricated using the precursor approach on lattice matched substrates. A modified precursor approach has also been developed for fabricating compounds containing high vapor pressure elements such as $\mathrm{Pb}{ }^{80}$

Figure 20, from Chang et al., ${ }^{79}$ shows the composition distribution of a doped $(\mathrm{Ba}, \mathrm{Sr}) \mathrm{TiO}_{3}$ thin film library. This was also the first study where quantitative scanning microwave microscopy (SMM) ${ }^{81}$ was used as an effective tool to scan and screen dielectric properties of thin film libraries. An important finding from this study was that high valence TM dopants such as $\mathrm{W}$ are effective in reducing the dielectric loss by minimizing the generation of oxygen vacancies during the crystallization process. A similarly designed library was also reported in this paper to study the effect of dopants on the properties of $(\mathrm{Ba}, \mathrm{Sr}) \mathrm{TiO}_{3}$ high- $\kappa$ capacitors with electrodes. It was found that the same high valence TM ions are instrumental in reducing the leakage current and boosting the breakdown voltage of the capacitors. Further research on single composition samples showed that $\mathrm{W}$ doping of $(\mathrm{Ba}, \mathrm{Sr}) \mathrm{TiO}_{3}$ thin films indeed results in reduced dielectric loss. 82

A recent surge of research activity in tunable microwave devices for phased array antenna and other communication devices has brought much attention to the microwave properties of ferroelectric thin films in general. The $100 \mathrm{MHz}$ to $100 \mathrm{GHz}$ (approximately) frequency band is a crucial range for these materials, where domain wall motion and dielectric relaxation associated with the soft phonon mode are known to occur. ${ }^{83}$ Thus, it is important to understand the frequency dependence of their dielectric (real and imaginary) constants. In one study, multifrequency mode variable temperature SMM was used to map the frequency (1-10 GHz), composition, and temperature dependence of $\mathrm{Ba}_{1-\mathrm{x}} \mathrm{Sr}_{\mathrm{x}} \mathrm{TiO}_{3}$ near the Curie temperature. ${ }^{84}$ The epitaxial $\mathrm{Ba}_{1-\mathrm{x}} \mathrm{Sr}_{\mathrm{x}} \mathrm{TiO}_{3}$ composition spreads were fabricated using PLD and the layer-by-layer

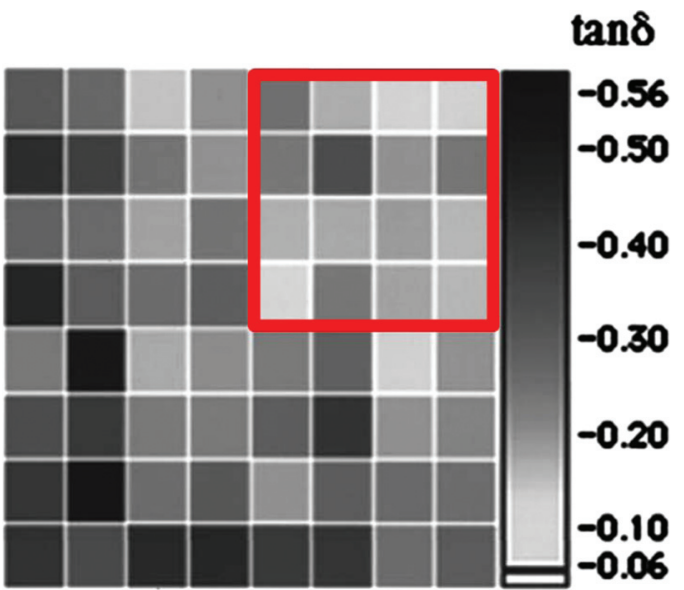

FIG. 20. Dielectric loss mapping of doped $\mathrm{BaTiO}_{3}$ films measured by scanning microwave microscopy. The region defined by the red box are compositions doped with W. The loss is low in general in this region. Reprinted with permission from H. Chang et al., Appl. Phys. Lett. 72, 2185 (1998). Copyright 1998 American Institute of Physics. 
gradient wedge approach similar to that depicted in Fig. 6. Figure 21, from Chang et al., ${ }^{85}$ shows the frequency dispersion of the dielectric constant, as measured by SMM. The fact that the broad peak is observed in the range $\mathrm{x}=0.2-0.4$, where the Curie temperature is closest to room temperature (RT), indicates that there is coupling between the microwave field and with the local soft mode mediated by the presence of structural defects such as threading dislocations.

Other combinatorial investigations of ferroelectric materials focused on chemical synthesis techniques for preparing libraries, ${ }^{86,87}$ reflecting the versatility of high throughput methodologies.

In searching for new materials with high piezoelectric coefficients, a particular emphasis is placed on identifying compositions at structural boundaries called morphotropic phase boundaries (MPBs). At MPBs, materials undergo structural phase transitions, and the MPB is vertical in a temperature-composition phase diagram, i.e., it is isocompositional. At such compositions, small perturbations in the material caused by electric field or stress can lead to an enhanced piezoelectric response. Thus, the most commonly used piezoelectric materials are at MPB compositions such as $\mathrm{PbZr}_{0.52} \mathrm{Ti}_{0.48} \mathrm{O}_{3}$ and $\left(\mathrm{PbMg}_{1 / 3} \mathrm{Nb}_{2 / 3} \mathrm{O}_{3}\right)_{0.7}-\left(\mathrm{PbTiO}_{3}\right)_{0.3}$. In a recent CCS library study, $\mathrm{BiFeO}_{3}$, an intrinsic multiferroic material that displays rich and complex physical properties was selected as a starting compound to screen for a novel lead free piezoelectric material, specifically at MPB compositions. ${ }^{88-90}$ A series of $\mathrm{Bi}_{1-\mathrm{x}}(\mathrm{RE})_{\mathrm{X}} \mathrm{FeO}_{3}$ pseudobinary CCS epitaxial libraries $(200 \mathrm{~nm}$ thick) were fabricated epitaxially at $600{ }^{\circ} \mathrm{C}$ on $\mathrm{SrTiO}_{3}$ (STO) substrates by layerby-layer PLD (see, e.g., Fig. 6).

Quantitative piezoresponse force microscopy (PFM) was performed to measure the out-of-plane piezoresponse. The PFM scan of a $\mathrm{Bi}_{1-\mathrm{x}} \mathrm{Sm}_{\mathrm{x}} \mathrm{FeO}_{3} \mathrm{CCS}$ library indicated substantial enhancement of the piezoresponse near the composition $\mathrm{Bi}_{\sim 0.8-0.85} \mathrm{Sm}_{\sim 0.2-0.15} \mathrm{FeO}_{3}$, where the structure was found by XRD to undergo significant change in the out of plane lattice constant. The effective piezoelectric coefficient values, $\mathrm{d}_{33}$, were then measured across the composition spread, ${ }^{89}$ Fig. 22. At $\mathrm{x}=0.13-0.15, \mathrm{~d}_{33}$ displays a rapid increase, peaking at $\mathrm{x}=0.14$; beyond this composition, $\mathrm{d}_{33}$

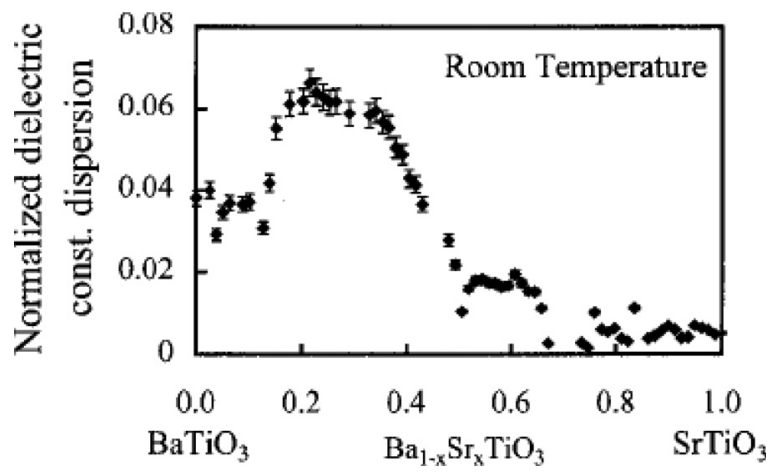

FIG. 21. Normalized dielectric constant dispersion vs. composition on a library sample, as measured by microwave microscope. The dispersion is defined as (dielectric constant at $0.95 \mathrm{GHz}$ - dielectric constant at $4.95 \mathrm{GHz}$ )/ dielectric constant at $0.95 \mathrm{GHz}$. The dispersion is largest in the range $\mathrm{x}=0.2-0.4$. Reprinted with permission from K. S. Chang et al., Appl. Phys. Lett. 79, 4411 (2001). Copyright 2001 American Institute of Physics.

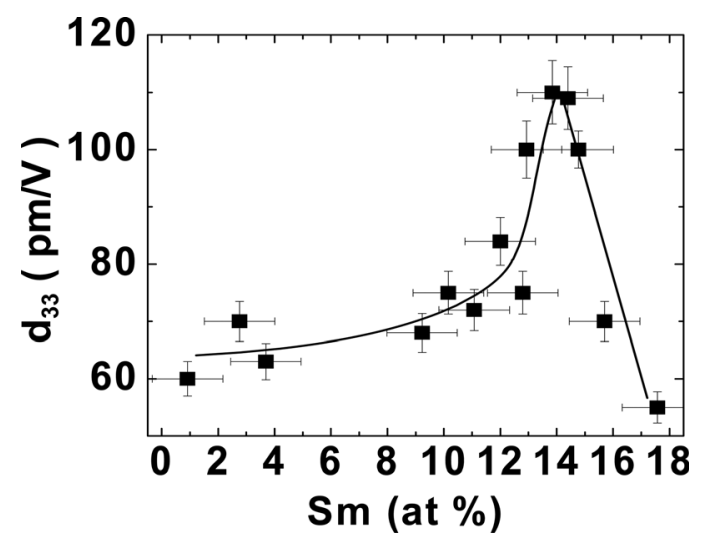

FIG. 22. Discovery of a lead free morphotropic phase boundary. The piezoelectric coefficient, $\mathrm{d}_{33}$, is mapped on $\mathrm{B}_{1-x} \mathrm{Sm}_{\mathrm{x}} \mathrm{FeO}_{3}$ composition spread. A strong peak is observed at $\mathrm{x}=0.14$. Reprinted with permission from $\mathrm{S}$. Fujino et al., Appl. Phys. Lett. 92, 202904 (2008). Copyright 2008 American Institute of Physics.

rapidly decreases. The measured remnant polarization, and high field $d_{33}$ in this case are similar to values previously reported for epitaxial thin films of the lead based MPB compounds mentioned above. Detailed microstructural studies have shown that the structural transition for this MPB is a rhombohedral to orthorhombic structural transition. Planar TEM of a single composition sample at $\mathrm{x}=0.14$ showed the presence of nanoscale twinned triclinic domains $20-50 \mathrm{~nm}$ in size. The occurrence of such nano twins and the stress accommodation associated with them is a signature of an adaptive ferroelectric phase at the MPB, which enables the high piezoelectric coefficient. ${ }^{91}$ Subsequent work has revealed that a chemical pressure effect, due to RE substitution into the Bi site (perovskite A-site), induces the structural transition and the concomitant enhancement in piezoelectric properties. $^{92}$

In another study, the B-site substitution effect on the ferroelectric properties in $(\mathrm{Bi}, \mathrm{Sm}) \mathrm{FeO}_{3}$ was investigated to systematically investigate the relative effects of A-site and B-site doping, using a pseudoternary composition spread. Sc was selected as the substitutional dopant for the B-site. ${ }^{93}$ The Sc ion has a $3^{+}$valence, which is not likely to cause an increase in leakage current. To make this library, the moving shutter technique (e.g., Fig. 6) was implemented in an epitaxial PLD process. On the triangularly shaped library, Fig. 23(a), the concentration of each dopant (A- or B-site dopant) varies along one side of the triangle. The three vertex compositions are $\mathrm{BiFeO}_{3}, \mathrm{Bi}\left(\mathrm{Fe}_{0.8} \mathrm{Sc}_{0.2}\right) \mathrm{O}_{3}$, and $\left(\mathrm{Bi}_{0.8} \mathrm{Sm}_{0.2}\right) \mathrm{FeO}_{3}$. In Fig. 23(a), all RT hysteresis loops taken across the spread are displayed. Ferroelectric properties exhibit clear changes in response to the substitution induced structural evolution. In the region near the $\mathrm{BiFeO}_{3}$ vertex, where the dopant composition is relatively low, unclosed loops are observed, indicative of high leakage current. As the substitution of Sm and Sc is increased, the square shaped ferroelectric hysteresis loop with a saturation polarization of about $70 \mu \mathrm{C} / \mathrm{cm}^{2}$ begins to appear, indicating that both substituted species lead to a reduction of the leakage current; an antiferroelectric phase may be playing a role in suppressing the leakage current. Comparison of Figs. 23(a) and 23(b) shows that the occurrence of the 


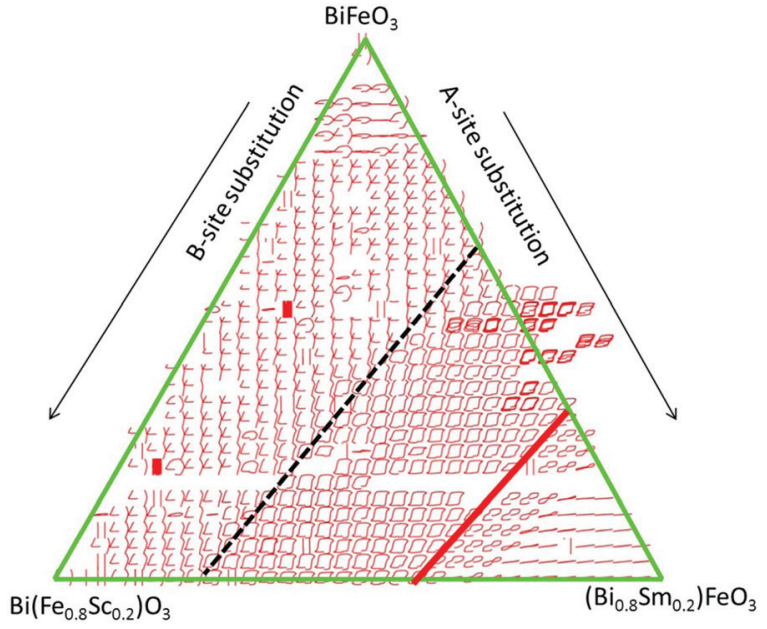

(a)

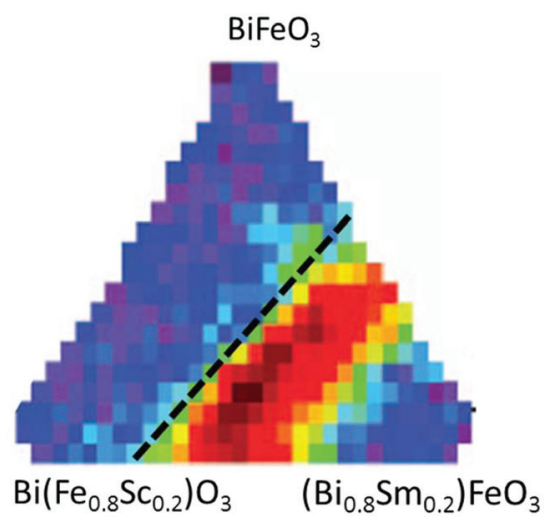

(b)

FIG. 23. (a) Ferroelectric and structural properties of a $\mathrm{BiFeO}_{3}$ $\mathrm{Bi}\left(\mathrm{Fe}_{0.8} \mathrm{Sc}_{0.2}\right) \mathrm{O}_{3}-\left(\mathrm{Bi}_{0.8} \mathrm{Sm}_{0.2}\right) \mathrm{FeO}_{3}$ pseudoternary composition spread. Ferroelectric hysteresis loops measured at each spot are plotted. The red line marks the MPB, where the square hysteresis transitions into double hysteresis loops. The dotted line is where the hysteresis loops become square shaped, indicating that the films are non-leaky, and (b) ferroelectric and structural properties of a $\mathrm{BiFeO}_{3}-\mathrm{Bi}\left(\mathrm{Fe}_{0.8} \mathrm{Sc}_{0.2}\right) \mathrm{O}_{3}-\left(\mathrm{Bi}_{0.8} \mathrm{Sm}_{0.2}\right) \mathrm{FeO}_{3}$ pseudoternary composition spread. Normalized intensity of the $1 / 4\{011\}$ superstructure spot, corresponding to the occurrence of an antiferroelectric secondary phase, is plotted as a function of position. The dotted line is the same composition line as the dotted line in Fig. 23(a), indicating that the presence of the antiferroelectric phase plays a role in controlling the leakage conduction in the films. Reproduced with permission from D. Kan et al., Integr. Ferroelectr. 111, 116 (2009). Copyright 2009 Taylor and Frances, Inc.

square-shaped hysteresis loops coincide with the composition region having the greatest superstructure spot intensity. Also, a closer look at the trend shows that in the composition region where only Sc is substituted, the hysteresis loops are characteristic of leaky ferroelectrics, indicating that $\mathrm{Sm}$ is more effective than $\mathrm{Sc}$ in reducing the leakage current. As the $\mathrm{Sm}$ concentration reaches $14 \%$, the ferroelectric hysteresis loops exhibit a transition to double hysteresis loops, clearly shown Fig. 23(a). The complexity of this ferroelectric system highlights the efficacy of the combinatorial approach for screening the entire composition space, and correlating structural and ferroelectric effects.

Bulk piezoelectric materials are required for many applications, and bulk composition spread techniques have been developed; ${ }^{94,95}$ such techniques can be used to investigate how microstructural properties change as a function of composition in bulk diffusion couple samples, where two end member compositions are hydrostatically compacted and sintered to induce diffusion and a resultant composition gradient. Fig. 24 shows the variation in the electric-field induced transition in a bulk ceramic composition spread, where a subtle substitution effect of a small amount of La and Fe into the A-site of $\mathrm{Pb}\left(\mathrm{Zr}_{0.6} \mathrm{Ti}_{0.4}\right) \mathrm{O}_{3}$ was systematically investigated. ${ }^{94}$ A bulk library has also been used to study the effect of processing temperature on the structural variation of piezoelectric materials. $^{96}$

The last decade has seen an explosion of research on multiferroic materials, which are defined as exhibiting two or more ferroic properties (ferroelectricity, ferromagnetism, and ferroelasticity) in a single material. Such materials can exhibit interesting coupling between the different ferroic properties, leading to intriguing novel device applications for sensors, actuators, tunable high frequency communication devices, and novel logic devices. ${ }^{97}$ Among different types of multiferroic materials, nanocomposite materials have been well studied, since they have the potential to realize RT device operation due to cross coupled ferroelectric/ ferromagnetic properties. To study the formation of nanocomposite multiferroic thin films, PLD based CCS libraries were designed to directly probe continuous "mixing" of a ferroelectric material and a ferromagnetic material on an MgO (100) substrate, ${ }^{98}$ Fig. 25. One end of the spread was ferroelectric $\mathrm{PbTiO}_{3}$, and the other end was the ferromagnetic insulator $\mathrm{CoFe}_{2} \mathrm{O}_{4}$. A modified version of the epitaxial layer-by-layer wedge deposition method was used to synthesize this library. ${ }^{99}$ SMM was used to map the dielectric constant, and RT superconducting quantum interference device (SQUID) microscopy the magnetization, as a function of
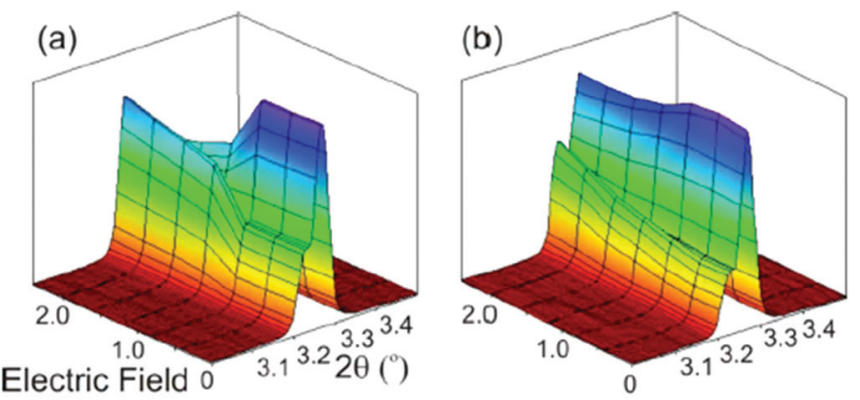

$(\mathrm{kV} / \mathrm{mm})$
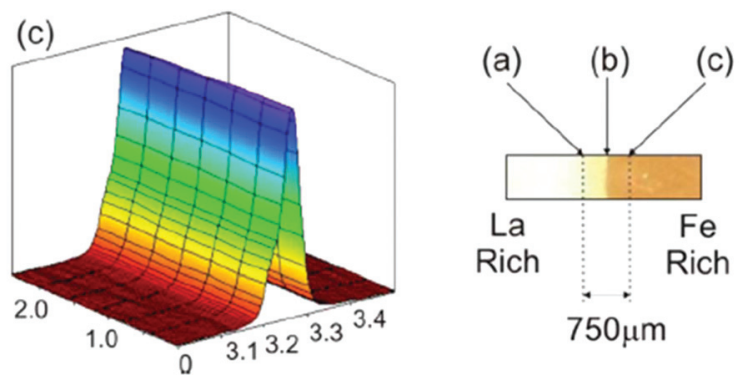

FIG. 24. Bulk composition spread. XRD patterns near the 111 reflections as a function of electric field amplitude. (a) La-rich region, (b) co-doped $\mathrm{La} / \mathrm{Fe}$ region, and (c) Fe-rich region of $\left(\mathrm{La}_{1-\mathrm{x}} \mathrm{Fe}_{\mathrm{x}}\right)_{0.02} \mathrm{~Pb}_{0.98} \mathrm{Zr}_{0.6} \mathrm{Ti}_{0.4} \mathrm{O}_{3}$. Vertical scale is linear intensity. Bottom right shows a photograph of the sample with the positions of the data points indicated. Reprinted with permission from J. L. Jones et al., Appl. Phys. Lett. 93, 152904 (2008). Copyright 2008 American Institute of Physics. 


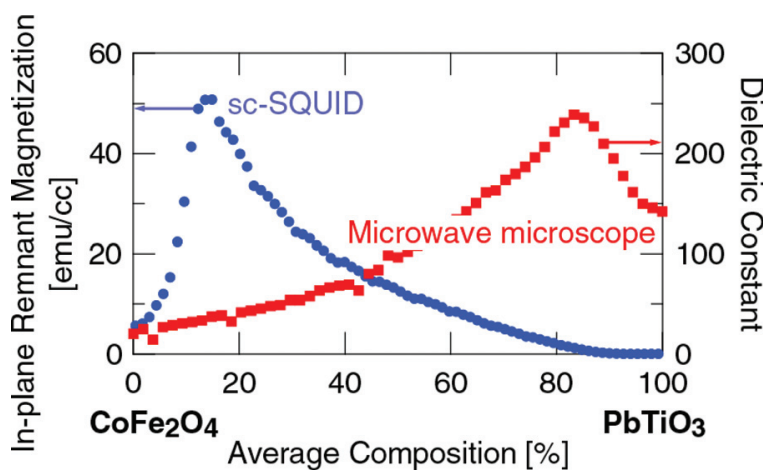

FIG. 25. Multiferroic properties of a $\left(\mathrm{CoFe}_{2} \mathrm{O}_{4}\right)_{1-\mathrm{x}}\left(\mathrm{PbTiO}_{3}\right)_{\mathrm{x}} \mathrm{CCS}$ library as a function of average percentage of $\mathrm{PbTiO}_{3}$. Remnant magnetization was measured by scanning SQUID microscopy and high dielectric constant region was mapped by microwave microscopy. Region in the middle was found to display coexisting magnetic-ferroelectric properties (replotted from data in Murakami et al ${ }^{98}$ ). Reprinted with permission from Murakami et al., Appl. Phys. Lett. 87, 112901 (2005). Copyright 2005 American Institute of Physics.

composition in the CCS library $\left(\mathrm{PbTiO}_{3}\right)_{1-\mathrm{x}}\left(\mathrm{CoFe}_{2} \mathrm{O}_{4}\right)_{\mathrm{x}}$. Figure 25, from Murukami et al., ${ }^{98}$ shows that there is a region in the middle of the spread, which displays coexisting magnetism and ferroelectricity (indicated by enhanced dielectric constant). A detailed microstructural investigation has revealed that different compositions displayed different nanocomposite nanostructures. In a follow-up study, SMM was used to measure the magnetoelectric coupling coefficient. ${ }^{100}$ The $\left(\mathrm{PbTiO}_{3}\right)_{1-\mathrm{x}}\left(\mathrm{CoFe}_{2} \mathrm{O}_{4}\right)_{\mathrm{x}}$ experiments demonstrated that one can study, using high throughput techniques, libraries of disparate crystal structures $\left(\mathrm{PbTiO}_{3}\right.$ is a perovskite, while $\mathrm{CoFe}_{2} \mathrm{O}_{4}$ is a spinel). This body of work also illustrates how one can explore the synthesis of multifunctional materials in general where the two end members may display a wide variety of functionalities beyond the three ferroic properties. Recently, a similar technique was used to systematically investigate synthesis of self-assembled vertical nanocomposites in the $\mathrm{BiFeO}_{3}-\mathrm{CoFe}_{2} \mathrm{O}_{4}$ system. ${ }^{101}$ Figure 26 shows the scanning electron microscopy (SEM) images taken at different average composition across the spread. Depending on the average composition, the strain in the film and the magnetization were found to continuously vary. This work showed that in such structures, magnetoelasticity dominates the magnetic properties, and illustrates how one can tune and design the microstructure and resulting properties of composites.

\section{MAGNETIC MATERIALS}

Magnetic phenomena have historically played profound roles in the advancement of human civilization; the technological foundation of modern society is built upon developments of magnetic materials. ${ }^{102}$ Today, magnetic materials and devices are ubiquitous in everyday life. For example, the steady rate at which the maximum energy product, $\left(\mathrm{BH}_{\max }\right)$, of permanent magnets has increased over the past decades led directly to continuous improvements in electric motor technology; further, magnetic memory, a key technology for information technology, recording and sensing devices is at the heart of a $\$ 100 \times 10^{9}$ industry.

Demand for higher performance magnetic materials in a wide range of technologies continues, and these applications represent ideal platforms for implementing combinatorial strategies. At the same time, fundamental scientific investigations into novel magnetic phenomena continue to uncover exotic effects such as multiferroicity and topological dielectricity. Exploration of such effects and their underlying physical mechanisms is directly tied to mapping of their phase diagrams. To this end, CCS experiments have been exploited to discover new compositions with improved properties, as well as to understand composition-structure-property relationships.

\section{A. High throughput magnetic characterization techniques}

A number of non-destructive scanning probe techniques have been used to map magnetic properties from combinatorial libraries. ${ }^{103-108} \mathrm{As}$ is the case for combinatorial measurements in general, a significant challenges lies in extracting accurate quantitative values of magnetic properties from a particular location on a library film, such that the value is in agreement with that obtained from a discrete
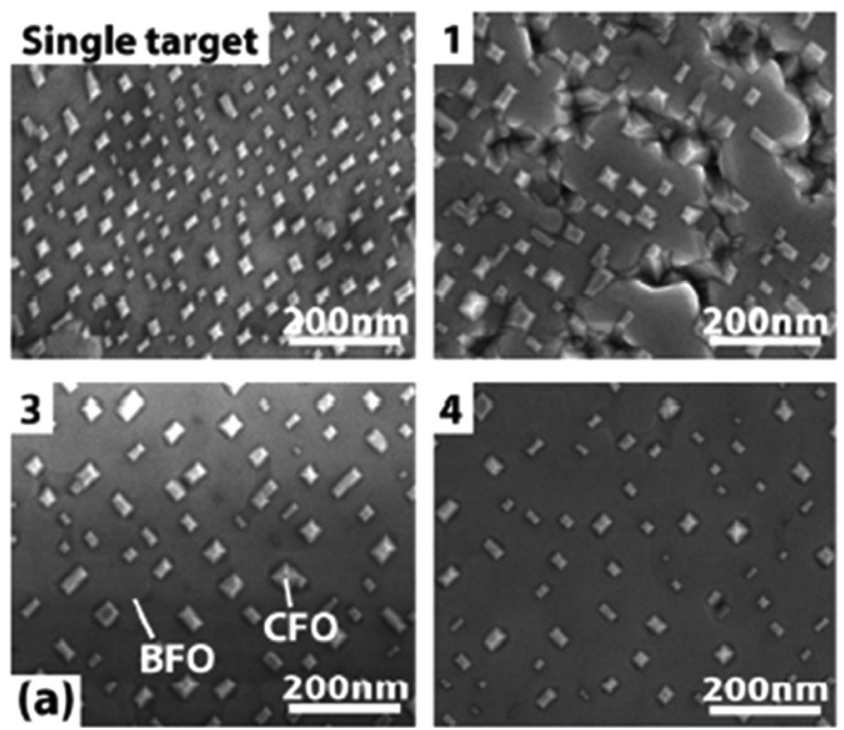
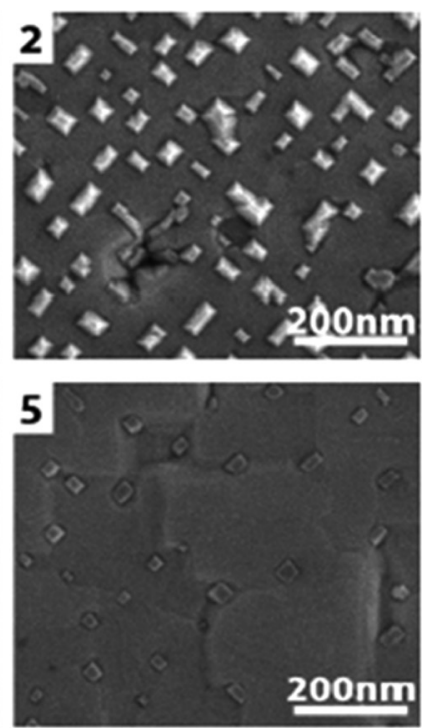

FIG. 26. SEM images of the top view of the film made from the single target and the composition spread samples of $\mathrm{BiFeO}_{3}$ (BFO)- $\mathrm{CoFe}_{2} \mathrm{O}_{4}$ (CFO) (1-5). As average composition ratio of $\mathrm{BFO}$ and $\mathrm{CFO}$ is varied, surface morphology is seen to change continuously. Reprinted with permission from N. M. Aimon et al., Appl. Phys. Lett. 100, 092901 (2012). Copyright 2012 American Institute of Physics. 
sample of the same composition, using a standard "bulk" measurement technique such as a SQUID magnetometer. Significant variations in some physical properties between bulk and thin film materials of the same composition are often the cause of the lack of agreement.

While there exist many important magnetic properties to be characterized, the net (i.e., remnant) magnetization is by far the most important property for screening of novel magnetic materials. Various techniques can be used to obtain magnetic field distributions emanating from thin film combinatorial samples. They include scanning SQUID microscopy $(\mathrm{SSM})^{104,107,108}$ and the scanning Hall probe technique. ${ }^{106}$ Even though magnetic field distribution itself does not yield the value of the magnetization, in principle, one can extract it using calibration samples. In the absence of an external applied field, remnant magnetization may be measured.

The magnetic-optical Kerr effect (MOKE) technique $\mathrm{e}^{103,105,106}$ is based on the fact that the polarization of light is rotated upon reflection from the surface of a magnetic material, and further that the rotation angle is directly proportional to the magnetization. MOKE is particularly useful since one can map the magnetic hysteresis loop from different positions on combinatorial samples by sweeping the applied magnetic field, from which one can obtain the saturation magnetization (through calibration) as well as the coercive field values. $^{103}$

The scanning Hall probe can also be used to map saturation magnetization values. ${ }^{106}$ In order to obtain absolute values of magnetization from the measured magnetic field without calibration, an inversion technique has been developed, which can be applied to any spatially resolved field distribution data. ${ }^{109}$ This technique uses a computational algorithm, which performs an inverse Fourier transform to calculate magnetic pole densities, which in turn is integrated to obtain magnetization values at any location on the library. This algorithm has been applied to analysis of RT SSM data from a variety of magnetic material libraries. ${ }^{107,108,110}$ In particular, it has been applied to thin film composition spreads of ternary metallic alloy systems containing ferromagnetic shape memory alloy (SMA) phases. The remnant magnetization values determined for the CCS library were demonstrated to be in good agreement with values measured by SQUID and vibrating sample magnetometers for single composition, large scale thin films. ${ }^{108}$

\section{B. Magnetic oxides}

Since the advent of high temperature superconductivity in layered perovskite cuprates, there has been an explosion of activity in all TM oxides. The rich variety of properties observed in metal oxides, such as colossal magnetoresistance, spin polarization, and long spin relaxation times, provides a versatile platform for exploring novel device applications.

Various sets of shadow mask designs have been designed for synthesis of pseudobinary and pseudoternary epitaxial CCS libraries, ${ }^{111,112}$ Fig. 27. This method was used $^{104}$ to fabricate epitaxial CCS libraries for metal oxide thin films on lattice matched substrates and enabled interrogation of the entire $\mathrm{La}_{1-\mathrm{x}} \mathrm{Sr}_{\mathrm{x}} \mathrm{MnO}_{3}$ composition space on a

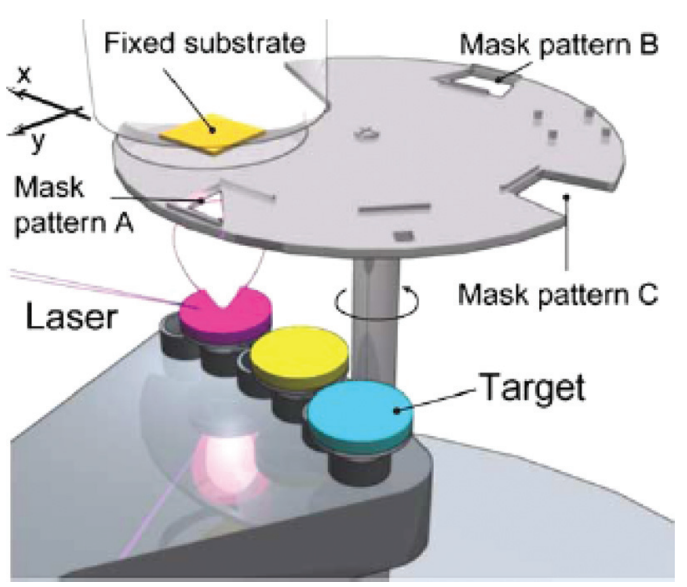

FIG. 27. Schematic for fabrication of ternary composition spreads using a set of shadow masks. The mechanism consists of three changeable targets, a fixed substrate, and a rotating mask system placed between them. The mask has three patterns, A, B, and C. The patterns A and B are used for ternary composition spread deposition, and the pattern $\mathrm{C}$ is for binary composition spread deposition. The $\mathrm{y}$ axis is along the radial direction of the mask rotation. Reproduced with permission from Y. Yamamoto et al., Appl. Surf. Sci. 223, 9 (2004). Copyright 2004 Elsevier BV.

single substrate, Fig. 28. The finding of infrared (IR) reflectivity at about $0.1 \mathrm{eV}$ is accompanied by a substantial increase in magnetization as $\mathrm{x}$ increases from 0.3 to a critical value of 0.5, as can be seen in Figs. 28(c)-28(e). This verifies the idea that a double exchange ferromagnetic interaction develops in a cooperative manner at a critical doping concentration range. From Figs. 28(a) and 28(b), the known magnetic-electronic phases can be identified in a straightforward manner. The phase diagram of Fig. 28(b) is in good agreement with the reported bulk phase diagram.

In a similar experiment, a precursor film synthesis technique for mapping the phase diagram of the $\mathrm{La}_{1-x} \mathrm{Ca}_{x} \mathrm{MnO}_{3}$ system on a single chip was employed. ${ }^{113}$ The CCS library chip was made by first depositing gradient thicknesses of the precursor layers $\mathrm{La}_{2} \mathrm{O}_{3}, \mathrm{Mn}_{3} \mathrm{O}_{4}$, and $\mathrm{CaMnO}_{3}$ at $\mathrm{RT}$ on a (100) $\mathrm{LaAlO}_{3}$ substrate, followed by annealing at $200{ }^{\circ} \mathrm{C}$ for several days, $400^{\circ} \mathrm{C}$ for $30 \mathrm{~h}$, and sintering at $1000^{\circ} \mathrm{C}$ for $2 \mathrm{~h}$ in flowing $\mathrm{O}_{2}$; a predominantly epitaxial library film resulted. To map the magnetic phases, low temperature SSM was carried out at $3 \mathrm{~K}$ to $7 \mathrm{~K}$, and to track the qualitative change in electrical properties of this CCS film, SMM was performed at $\mathrm{RT}^{81}$ The various property maps of the $\mathrm{La}_{1-x} \mathrm{Ca}_{x} \mathrm{MnO}_{3}$ library are shown in Fig. 29. The correlation between microwave, optical, and magnetic properties at low temperatures reveals "boundaries" between composition regions where properties change abruptly. The phase boundaries observed here were found to coincide with predicted boundaries that describe the spin order in the system as a function of composition using a generalized Hubbard model. ${ }^{114}$ At $x=0.5$, there is an abrupt change in the optical image, where $\Delta B_{z}$ exhibits a minimum. This composition is also believed to display half charge ordering, i.e., the $\mathrm{Mn}$ orbitals are ordered in the structure because they are evenly split between +3 and +4 valences. ${ }^{113}$ At $x=0.93$, where $\Delta B_{z}$ reaches another minimum, the optical image and the SMM data abruptly change in a concomitant manner. This is believed to be a transition to an electrically insulating 


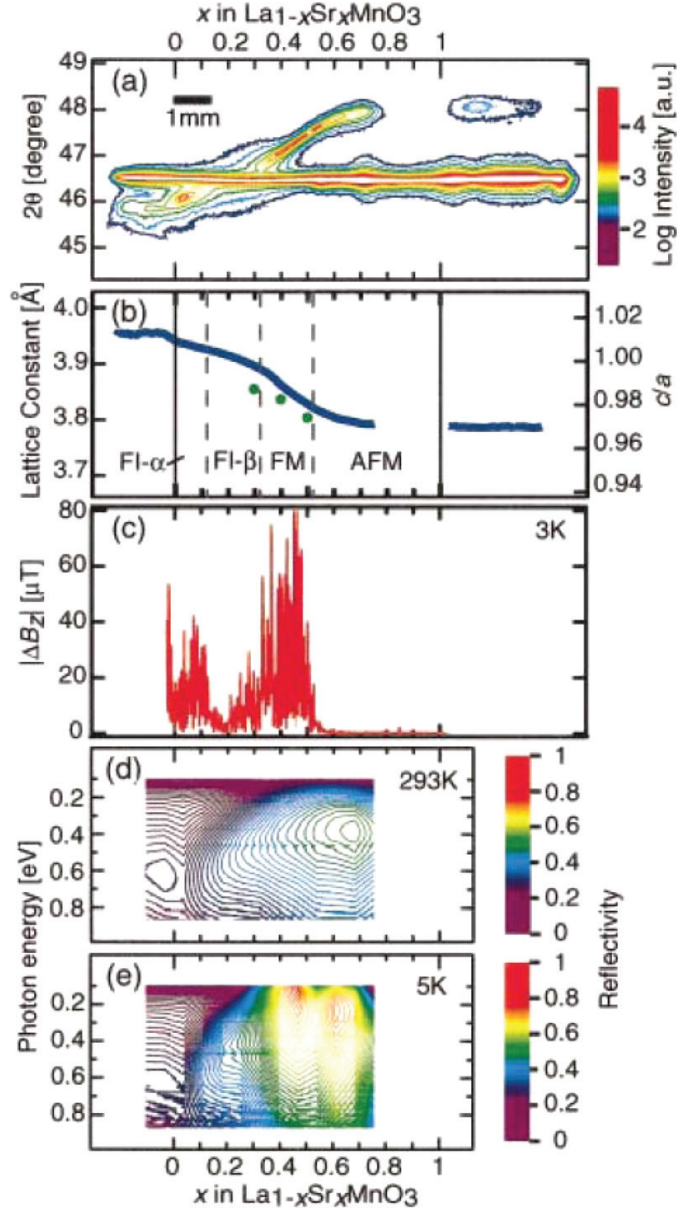

FIG. 28. Structural-magnetic-electronic properties of a $\mathrm{La}_{1-x} \mathrm{Sr}_{x} \mathrm{MnO}_{3} \mathrm{CCS}$ library as a function of $\mathrm{x}$. (a) A concurrent XRD spectra ( $\theta / 2 \theta$ scan) at RT. The perpendicular axis represents the $2 \theta$ angle. The colored contour lines denote logarithmic intensity of the XRD peak. The scale bar denotes length on the film along the $\mathrm{x}$ axis. (b) The out of plane lattice constants calculated from (a) (blue curve). (c) The strength of the out of plane magnetic field $|\triangle \mathrm{Bz}|$ measured with scanning SQUID microscope at $3 \mathrm{~K}$. (d) The contour map of infrared reflectivity at $293 \mathrm{~K}$ and (e) at $5 \mathrm{~K}$. Color bars denote magnitude of the reflectivity. Reprinted with permission from T. Fukumura et al., Appl. Phys. Lett. 77, 3426 (2000). Copyright 2000 American Institute of Physics.

antiferromagnetic state. Magnetic domain distributions measured at several spots on the library by low temperature scanning SQUID are also in agreement with the changing magnetic states across the phase diagram.

Tracking magnetic transitions as a function of composition is an extremely important aspect of mapping magnetic phase diagrams. Direct simultaneous visualization mapping of the magnetic transition across an entire composition spread sample was demonstrated using magneto-optic imaging with an indicator film. ${ }^{115}$ By taking an image of the library spread film with an indicator film over it, Fig. 30, one can readily identify the ferromagnetic region, which exhibits a dark edge due to stray field (in the case of an in-plane aligned film). By tracking the changing width and position of the dark edge as a function of temperature, one can easily "see" the Curie temperature of the entire phase diagram, schematically shown in Fig. 31 for the case of a $\mathrm{La}_{1-x} \mathrm{Ca}_{x} \mathrm{MnO}_{3}$ library film. ${ }^{115}$ The Curie temperature mapping across this sample (diamonds) is superimposed over a previously published bulk phase

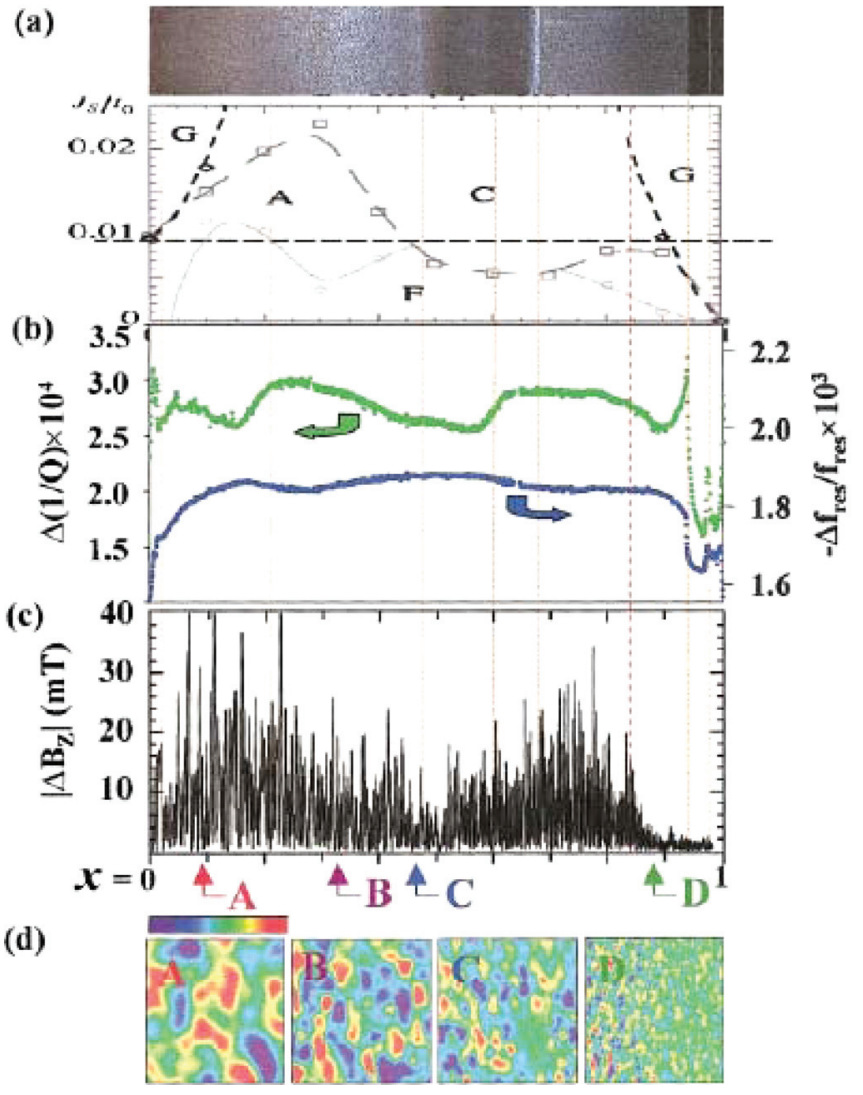

FIG. 29. Electronic and magnetic transitions in the $\mathrm{La}_{1-\mathrm{x}} \mathrm{Ca}_{\mathrm{x}} \mathrm{MnO}_{3}$ pseudobinary system studied using SEMP and scanning SQUID microscope: (a) a RT charge-coupled device (CCD) photograph of the continuous $\mathrm{La}_{1-\mathrm{x}} \mathrm{Ca}_{\mathrm{x}} \mathrm{MnO}_{3}$ thin film taken under white light and a magnetic phase diagram of the system determined from bulk single crystals (the various states are: paramagnetic insulator (PI), ferromagnetic insulator (FI), ferromagnetic metal (FM), charge-ordered insulator (COI), and antiferromagnetic insulator (AFI)); (b) SEMP line-scan profiles of microwave loss $\Delta 1 / \mathrm{Q}$ (related to conductivity) and relative frequency shift $\Delta \mathrm{f} / \mathrm{f}$ (measurement performed at RT); (c) scanning SQUID line profile obtained at $3 \mathrm{~K}$ (the oscillation indicates magnetic domains); and (d) scanning SQUID images taken at $7 \mathrm{~K}$ at four different composition regions showing the magnetic domains and the transition from strong to weak magnetization. Reproduced with permission from Y. K. Yoo et al., Phys. Rev. B 63(22), 224421 (2001). Copyright 2001 American Physical Society.

diagram, where various acronyms indicate different magnetic states. Although shifted compared to the bulk curve, one can clearly discern the transition curve from the paramagnetic to the ferromagnetic state, as well as the sharp composition boundary at $x=0.5$ between the ferromagnetic and antiferromagnetic states. Because thin film samples are known to exhibit property deviations compared to bulk samples due to strains and presence of defects, etc., the observed shift is not unreasonable. This experiment clearly illustrates that one can use a simple technique to map Curie temperature across composition spread libraries. In another example, the above mentioned magneto-optical technique has been used to successfully map ultraviolet (UV)/visible light properties in the spinel system $(\mathrm{Zn}, \mathrm{Co}) \mathrm{Fe}_{2} \mathrm{O}_{4}{ }^{116}$

One of the most powerful examples of the efficacy of combinatorial methodologies is the discovery of magnetic semiconducting oxides in $\mathrm{Co}-\mathrm{TiO}_{2} \cdot{ }^{117}$ Ferromagnetic semiconductors, formed by doping magnetic impurities into host semiconductors, are key materials for spintronics, where the 

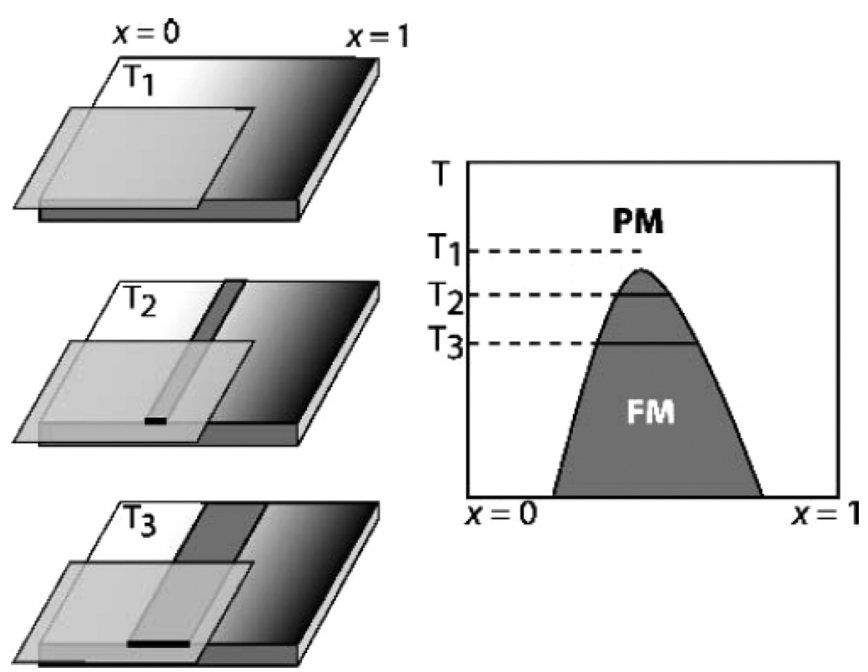

FIG. 30. Schematic showing how a magnetic phase diagram is constructed using magneto-optic imaging using an indicator film technique). The length and location of dark lines at different temperatures indicate a magnetic phase region on a composition-temperature diagram. Reproduced with permission from M. J. Turchinskaya et al., J. Mater. Res. 19, 2546 (2004). Copyright 2004 Materials Research Society.

correlation between the spin and the charge of electrons leads to spin dependent functionalities. Previously, most known magnetic semiconductors had been non-oxides. Matsumoto et al. ${ }^{118}$ fabricated thin film libraries of $3 \mathrm{~d}$ TM doped $\mathrm{TiO}_{2}$ thin films. When they were screened with an SSM, Co doped $\mathrm{TiO}_{2}$ was found to exhibit ferromagnetism at RT while maintaining optical transparency, in a single phase microstructure, up to $8 \% \mathrm{Co}$. This high throughput discovery gave birth to the field of magnetic semiconducting oxides; since this discovery, exploration for novel magnetic semiconducting oxides in a variety of materials systems has become an international research endeavor. High-throughput experimentation continues to play an important role in deciphering the exact mechanism by which doped magnetic species brings about ferromagnetism. ${ }^{119,120}$

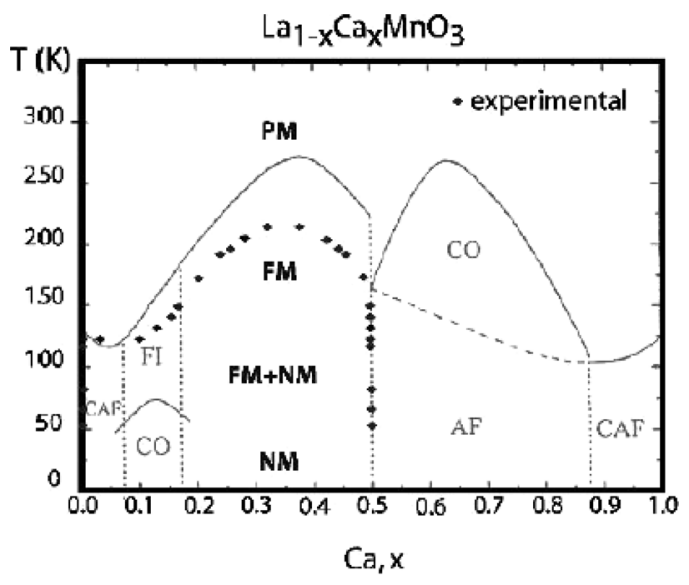

FIG. 31. Constructed magnetic phase diagram of the $\mathrm{La}_{1-x} \mathrm{Ca}_{x} \mathrm{MnO}_{3}$ composition spread on a $\mathrm{SrTiO}_{3}$ substrate). The experimental points (diamonds) are superimposed on the bulk phase diagram. PM, FM, NM, AF, CAF, CO, and FI refer to paramagnetic, ferromagnetic, non-magnetic, antiferromagnetic, canted antiferromagnetic, charge ordered, and ferromagnetic insulating states, respectively.

\section{Magnetic metallic alloys and magnetic phase diagrams}

The search for novel magnetic materials lead to some of the original high throughput experiments and techniques. ${ }^{13,121}$ Magnetic alloys were screened for several different functional properties, targeting specific applications such as recording heads and inductor cores, using co-sputtering techniques to rapidly perform phase mapping. In one example, ${ }^{121}$ the $\left(\mathrm{Ni}_{x} \mathrm{Fe}_{y}\right)_{1-x}\left(\mathrm{SiO}_{2}\right)_{x}$ pseudoternary system was co-sputtered using the segmented target approach. The schematic in Fig. 32 shows the three disk segments $\left(\mathrm{Ni}, \mathrm{Fe}\right.$, and $\left.\mathrm{SiO}_{2}\right)$ placed on one radio frequency (RF) sputtering target electrode. Analysis of the deposition profile resulting from the co-sputtering of the three segments indicated that a large fraction of the phase diagram of this pseudoternary system could be achieved in the library film. Resistance, magnetoresistance, permeability, and magnetization were measured across the CCS library; the latter was measured by MOKE. At least, one other example of magnetic properties mapped as a function of composition for a codeposited CCS library exists. ${ }^{122}$ A different approach for fabricating CCS films has been reported by Priyadarshini et al., ${ }^{123}$ by using a combination of evaporation filaments to co-deposit alloy libraries containing as many as four elements. Because the filaments do not take up much space, the library can be deposited in an area less than $0.5 \mathrm{in}$. in diameter, as compared to typical CCS libraries fabricated by co-sputtering, which are 3 in. in diameter or larger.

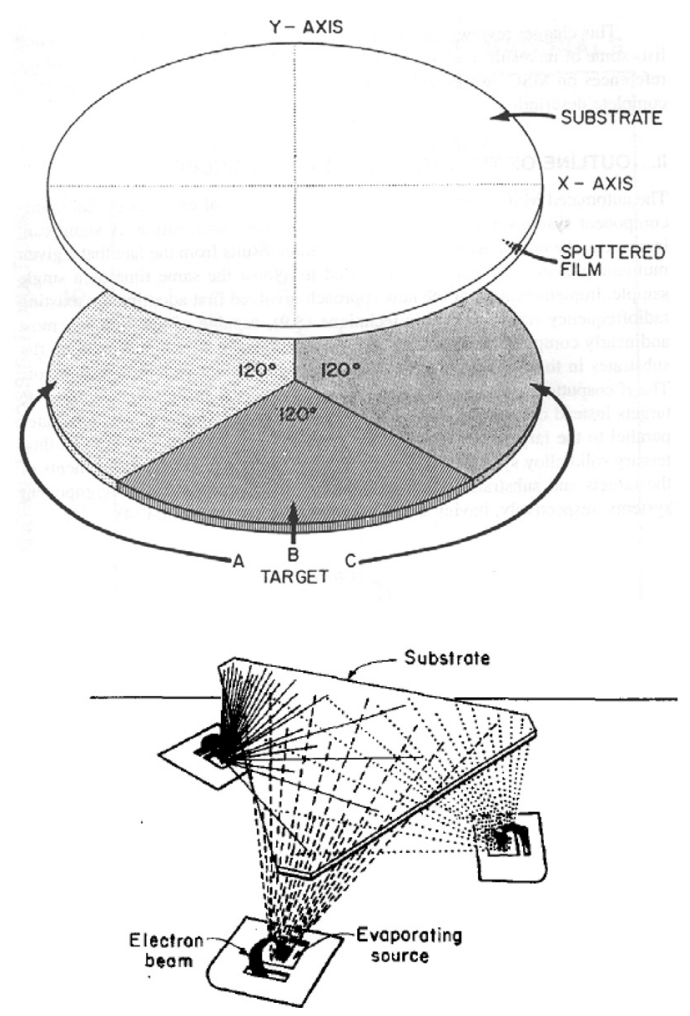

FIG. 32. Schematic illustration of two methods for fabricating combinatorial thin films: (a) co-sputtering from a three component sputtering target to map phase diagrams. Reproduced with permission from J. J. Hanak, J. Mater. Sci. 5, 964 (1970). Copyright 1970 Springer; and (b) co-evaporation system using electron beam vapor deposition for mapping a ternary phase diagram. Reprinted with permission from K. Kennedy et al., J. Appl. Phys. 36, 3808 (1965). Copyright 1965 American Institute of Physics. 
To date, less than $5 \%$ of all possible ternary metal phase diagrams have been investigated; even fewer systems exist for which magnetic properties have been mapped. Phase diagrams mapped using thin CCS film libraries must be regarded as "thin film phase diagrams." Composition-structure-property relationships deduced from thin film libraries can vary from those obtained using bulk samples. Importantly, most high technology devices comprise highly integrated multilayer thin film structures, and thin film phase diagrams are often more relevant than bulk phase diagrams in understanding and modeling these devices. While mapping of magnetic properties alone can provide great insight, correlating these properties with phase structure across composition is much more valuable. Such correlations have only been recently carried out; the key facilitating factors for such an integrated approach are the advent of computer technology and the use of sophisticated measurement instrumentation. A good example of comprehensive mapping of the structural and magnetic properties of a phase diagram using the CCS spread method is the experiment of Yoo et al. ${ }^{124}$ The Fe-Ni-Co system was deposited onto a triangular sapphire substrate by sequential ion beam deposition of the elemental metals at RT, followed by extensive annealing in vacuum $\left(600^{\circ} \mathrm{C}\right.$ at $10-8$ Torr $)$ to allow complete interdiffusion of the layers and appropriate phase crystallization. XRD was used to obtain the structural phase distribution. A $4^{\circ} 2 \theta$ range $\left(42^{\circ}-46^{\circ}\right)$ contained all of the major diffraction features in the phase distribution. For a given structural phase, the X-ray peak intensity allows one to track the compositional range. Regions in which phases coexist could be identified by looking for diffraction peaks arising from multiple phases. It was observed that near the phase boundaries, the full width half maximum of the peaks tended to increase. Figure 33(a) shows the distribution of phases deduced from the library sample; fcc $\mathrm{Ni}$, bcc Fe, and fcc and bcc Ni-Fe alloys are present. This result is consistent with the known bulk structural phases in this alloy system. The magnetic properties of the library film were investigated by MOKE, Fig. 33(b). A qualitative saturation magnetic moment of each point of the ternary phase diagram was mapped at applied magnetic fields of $+/-50$ Oe. Comparison of the two figures shows a clear correlation between the phase distribution and the magnetization; the bcc Fe has the highest magnetization. Further, the observed magnetization variation is consistent with the known distribution of saturation induction of the Fe-Ni-Co system deduced using the traditional bulk method, Fig. 33(c). ${ }^{125}$ Although MOKE analysis does not allow direct quantitative measurement of magnetization, by comparing the measured Kerr rotation angle with values obtained on calibration samples using standard methods such as SQUID magnetometry, one can convert the Kerr angle map to a magnetization map. In this particular ternary experiment, a mechanical hardness map was also obtained using nanoindentation, which revealed that the Fe-Co region, consisting of fcc and bcc (two phase) or pure bcc (single phase) areas, is harder than the higher Ni concentration fcc region.

Recently, ternary systems containing Heusler alloys such as $\mathrm{Ni}_{2} \mathrm{MnGa}$ and $\mathrm{Co}_{2} \mathrm{MnGe}$ have attracted much attention due to their unique functional properties, such as the ferromagnetic shape memory effect and high spin polarization. ${ }^{108,126}$ In such alloy systems, it is of interest to understand how the compositional variation and structural properties affect the magnetic properties. The ferromagnetic shape memory effect can occur in ferromagnetic alloys that also display a reversible martensitic transition. In one study,
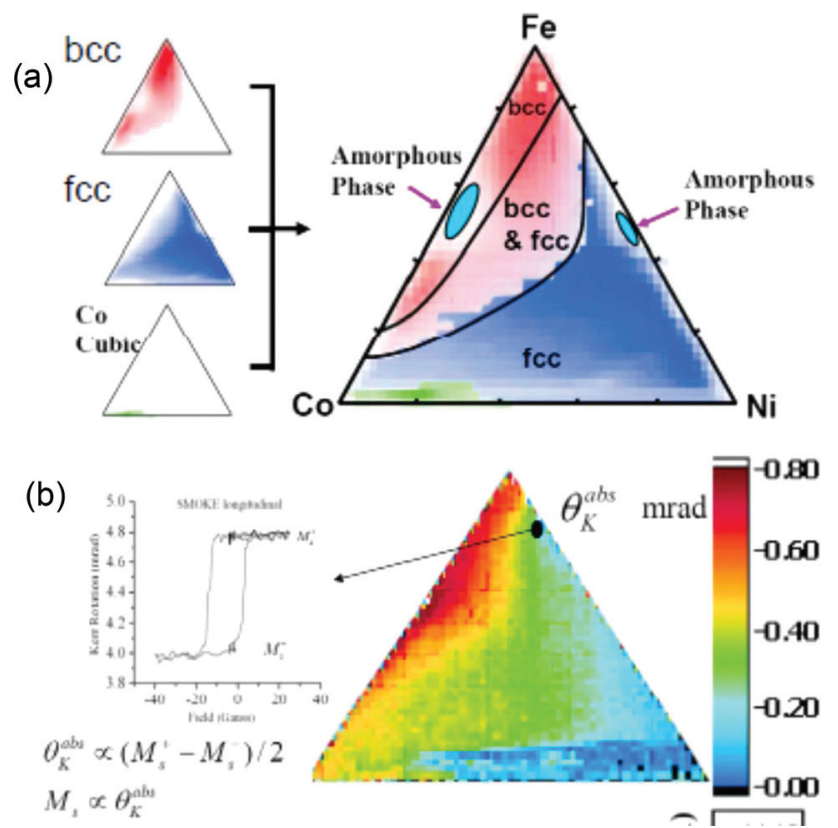

(c)

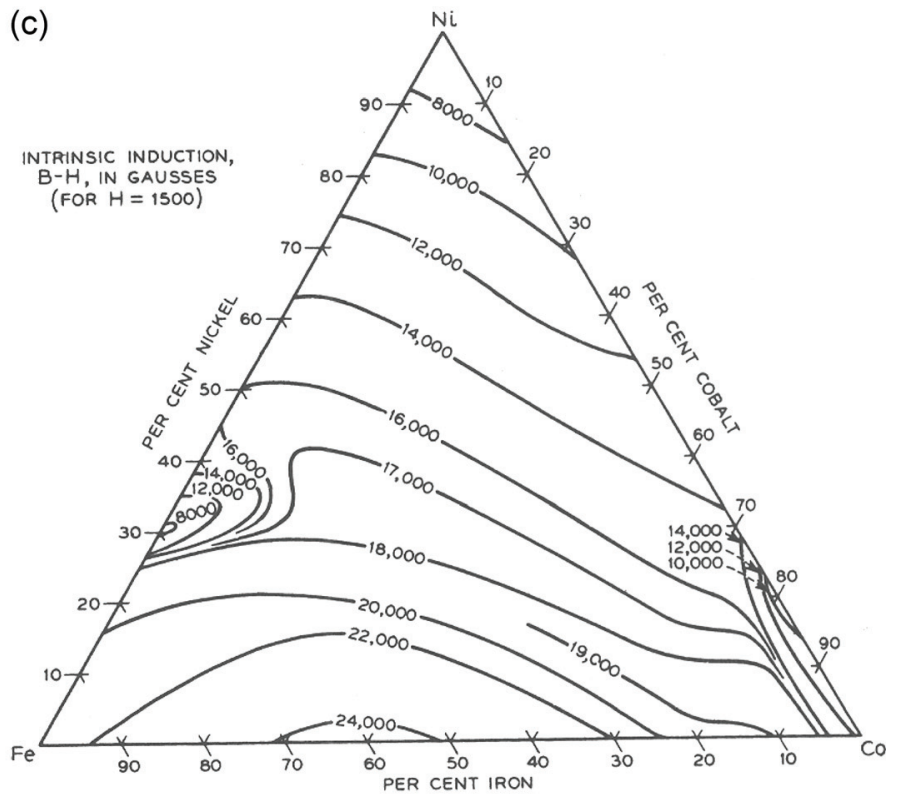

FIG. 33. Mapping of properties of a Fe-Ni-Co combinatorial thin film library: (a) the Fe-Ni-Co phase diagram obtained from distribution of structural phases deduced from X-ray microdiffraction peaks of fcc, bcc, and "Co cubic" phases, and (b) mapping of magnetic property using scanning magneto-optical Kerr effect measurement. The Kerr rotation angle measured at $+/-50$ Oe applied field is plotted. The figure at left shows a typical hysteresis loop. Reproduced with permission from Y. K. Yoo et al., Intermetallics 14, 241 (2006). Copyright 2006 Elsevier BV. (c) Saturation induction mapped for the Fe-Ni-Co ternary phase diagram using the traditional individual alloys for comparison. Reproduced with permission from R. M. Bozorth, Ferromagnetism (Wiley-IEEE Press, New Jersey, 1993). Copyright 1993 Wiley-IEEE Press. 
structural properties of the Ni-Mn-Ga system were measured in order to map the ferromagnetic SMA composition regions, to understand the origin of the coexistence of these two properties, ${ }^{108}$ Fig. 34. CCS libraries were deposited in an ultrahigh vacuum (base pressure $\sim 10-9$ Torr) magnetron cosputtering system. $\mathrm{Ni}, \mathrm{Mn}$, and $\mathrm{Ni}_{2} \mathrm{Ga}_{3}$ were used as the three targets. XRD of the fabricated films revealed that they were textured, with the (110) orientation normal to the substrate. WDS was used to accurately map the composition variation of every wafer. It was confirmed that by adjusting the power applied to each cathode, and the gun-substrate distance (typically $12 \mathrm{~cm}$ ), different regions of the ternary phase diagram (as well as the entire ternary diagram) could be mapped reproducibly. ${ }^{127}$ A RT SSM was used for rapid identification of ferromagnetic regions, with conversion of the field distribution information into quantitative magnetization values. ${ }^{109}$ The result of this calculation was plotted to obtain an RT map of remnant magnetization across the phase diagram, Fig. 34(a). In the figure, the solid triangle-shaped curve marks the compositional region mapped on this particular library film. The most strongly magnetic region stretches from near the middle of the phase diagram toward the slightly Ni-rich region. The circle indicates the region surrounding the Heusler composition, $\mathrm{Ni}_{2} \mathrm{MnGa}$, which had previously been extensively studied. ${ }^{128-130}$ Thus, it was shown that this composition is only one part of a large region exhibiting a high magnetization. As one moves away from this general region, the remnant magnetization decreases. The magnetization peak is observed near the center of the phase diagram, around the half Heusler composition NiMnGa. The RT magnetization
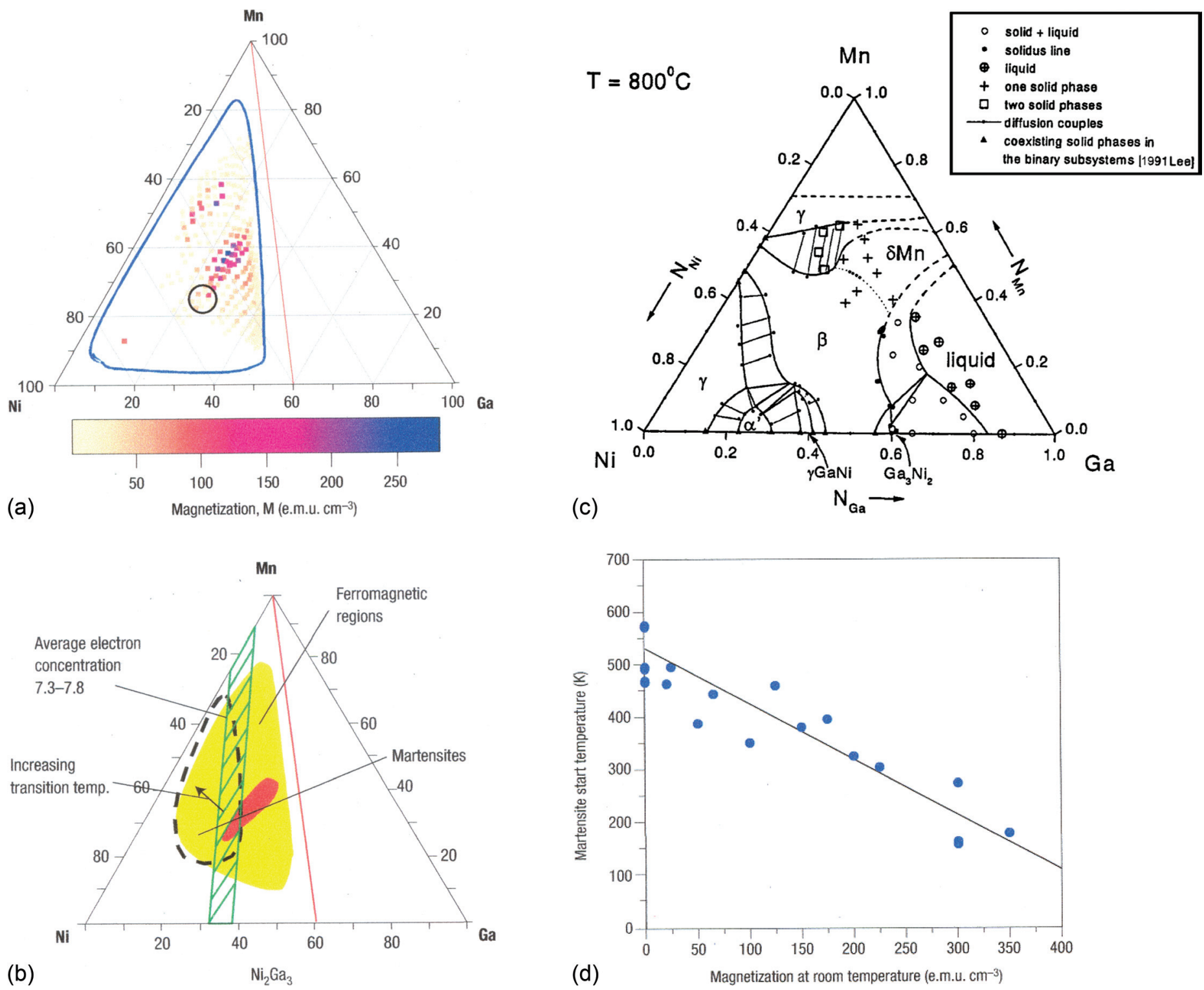

FIG. 34. Exploration of magnetic SMAs in the Ni-Mn-Ga ternary system. (a) RT mapping of remnant magnetization of the Ni-Mn-Ga system. (The region inside the triangular shaped curve is the compositional region mapped on a CCS library film; the circle marks the compositions near the $\mathrm{Ni}_{2} \mathrm{MnGa}_{\mathrm{Heusler}}$ composition); (b) superimposed functional phase diagram deduced from magnetic mapping and SMA mapping (the shaded region has the compositions with average electron/atom ratio 7.3-7.8; the dotted line surrounds the region of SMAs; in the ferromagnetic region, the red area has the highest magnetization); (c) $800^{\circ} \mathrm{C}$ isothermal section of the Ni-Mn-Ga ternary system; (d) Martensitic transformation temperature plotted against RT saturation magnetization (Each data point correspond to a composition on the spread wafer. The line is a linear fit to the data). Reprinted (Figs. 34(a), 34(b), and 34(d)) with permission from I. Takeuchi et al., Nature Mater. 2, 180 (2003). Copyright 2003 Macmillan Publishers Ltd. 
values extracted here, $\sim 200-300 \mathrm{emu} / \mathrm{cm}^{3}$, are consistent with saturation magnetizations obtained using a vibrating sample magnetometer on single composition bulk samples. In the same experiment, CCS libraries were deposited on wafers containing micromachined arrays of cantilevers to map the SMA transition temperature regions. Figure 34(b) summarizes the magnetic and the martensite phase diagrams deduced from the combined data. It is clear that there is a large region outside of the near Heusler composition that exhibits both ferromagnetic and reversible martensite behaviors. It has been shown that this class of material exhibits a martensitic instability for stoichiometries where the $(\mathrm{s}+\mathrm{d}$ electron)/atom ratio is $\sim 7.4 .^{128,129}$ The shaded area in Fig. 34(b) covers the composition region where the $(\mathrm{s}+\mathrm{d}$ electron)/atom ratio is 7.3-7.8, and a large overlap between the shaded area and the observed reversible martensite region can be observed. The RT magnetization is plotted against the martensitic start temperature in Fig. 34(d) for each composition in the large composition range studied. A clear trend between the two parameters emerges: the higher the magnetization, the lower the transformation temperature. From separate measurements, it has been confirmed that magnetization at RT is roughly proportional to the Curie temperature. The plot indicates that there is a strong thermodynamic magnetostructural coupling in this system. Such a coupling had previously been observed in only a limited compositional range near $\mathrm{Ni}_{2} \mathrm{MnGa}$ (inside the circle in Fig. 34(a). ${ }^{130}$ This experiment revealed that the relationship holds in general for magnetic alloys with a martensitic instability. A comparison of Fig. 34(b) with the $800^{\circ} \mathrm{C}$ isotherm of the Ni-Mn-Ga system Fig. 34(c) (Ref. 131)) shows that it is the $\beta$ phase, which largely transforms martensitically at low temperatures. Micro XRD of the spread indicated that much of the studied regions have the L2 $2_{1}$ Heusler structure, or a distorted version of it.

Another Heusler alloy, $\mathrm{Co}_{2} \mathrm{MnGe}$, was predicted to be fully spin polarized at the Fermi level. Although to date the spin polarized behavior has not been confirmed, the possibility that deviations from stoichiometry could cause a reduction in spin polarization has motivated ternary mapping of the Co-Mn-Ge system. ${ }^{105,132,133}$ The MOKE imaging technique was used to rapidly characterize the magnetic properties of the entire ternary system. Figure 35 shows the transverse differential MOKE intensity image of a $25 \mathrm{~nm}$ thick continuous ternary phase diagram chip measured using a charge couple display (CCD) camera while applying a magnetic field of $+/-5$ KOe. In this figure, areas of high intensity correspond to magnetic regions at RT with strong magneto-optic coupling. Triangularly cut $\mathrm{Ge}$ substrates of different orientations, Fig. 36, were used to map the ternary phase diagram using molecular beam epitaxy to deposit library films. Sub-monolayers of Co, Mn, and Ge were deposited sequentially using a combination of automated moving shadow masks, sample rotation, and source shutters. ${ }^{134}$ Three regions in the phase diagram in Fig. 35 were determined to be strongly ferromagnetic: (1) near pure Co, (2) near the Co-rich region centered around $\mathrm{Co}_{0.7} \mathrm{Mn}_{0.1} \mathrm{Ge}_{0.2}$, and (3) in the middle of the phase diagram, approximately at $\left(\mathrm{Co}_{0.5} \mathrm{Mn}_{0.5}\right)_{1-\mathrm{x}} \mathrm{Ge}_{\mathrm{x}}$, for $\mathrm{x}$ between 0.2 and 0.6. The area showing strong signals along the $\mathrm{Mn}_{\mathrm{x}} \mathrm{Ge}_{1-\mathrm{x}}$ binary edge, for

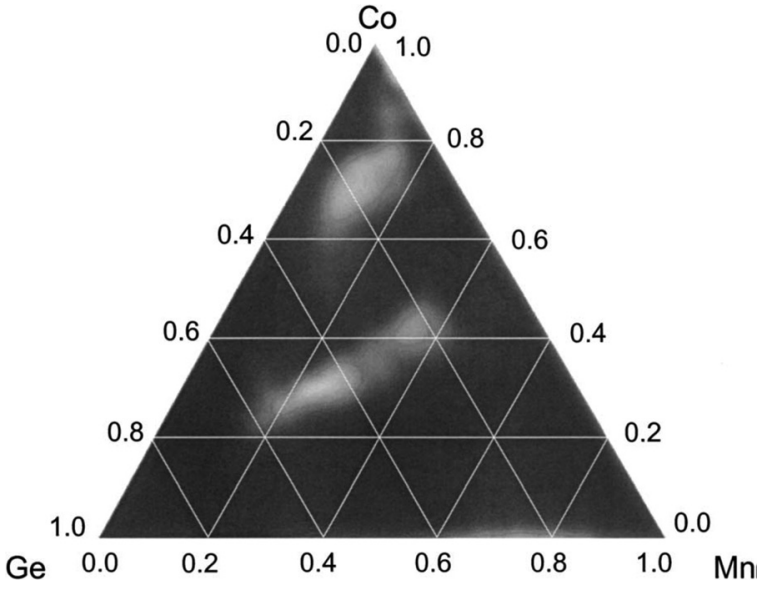

FIG. 35. RT magnetic phase diagram of a $25 \mathrm{~nm}$ thick Co-Mn-Ge phase diagram sample grown by molecular beam epitaxy (MBE). Image of differential MOKE intensity measured at $+/-5 \mathrm{Oe}$, which corresponds to the saturated states of the system. Reproduced with permission from F. Tsui and P. A. Ryan, Appl. Surf. Sci. 189, 333 (2002). Copyright 2002 Elsevier BV.

$\mathrm{x}$ between 0.6 and 0.9 , corresponds to a known antiferromagnetic region. Results are in good agreement with earlier reports on limited composition ranges inside the ternary system. In order to zoom in to study the details of magnetism in the $\left(\mathrm{Co}_{1-\mathrm{x}} \mathrm{Mn}_{\mathrm{x}}\right)_{0.8} \mathrm{Ge}_{0.2}$ region, a separate CCS library with a smaller composition range was synthesized. Reflection high (a)

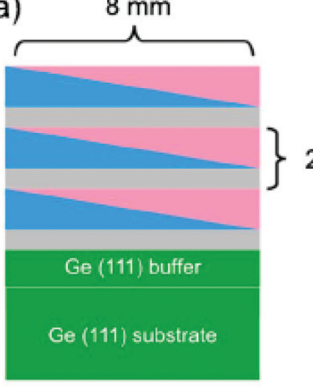

(c)

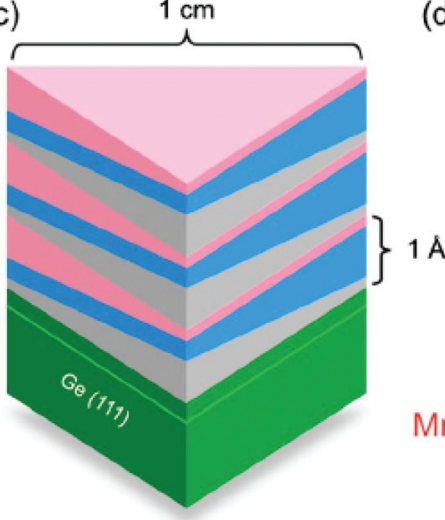

(b)

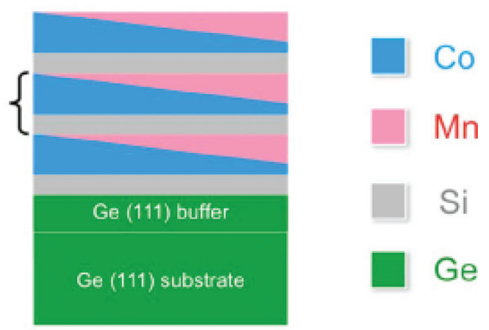

(d)

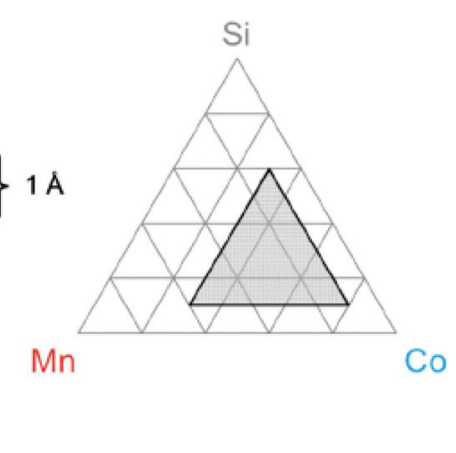

FIG. 36. Synthesis schemes of CCS libraries. Cross-sectional schematic views of (a) full binary sample $\left(\mathrm{Co}_{\mathrm{x}} \mathrm{Mn}_{1-\mathrm{x}}\right)_{\mathrm{a}} \mathrm{Si}_{1-\mathrm{a}}$ with $0 \leq \mathrm{x} \leq 1$ and (b) partial binary sample with $0.3 \leq \mathrm{x} \leq 1$. (c) Partial ternary sample and (d) composition range of the partial ternary sample (shaded region) within the full ternary phase diagram. The trilayers of Si-Co-Mn submonolayer wedges and "partial" wedges are typically repeated 200 times. Reprinted with permission from L. He et al., J. Vac. Sci. Technol. B 29, 03 C124 (2011). Copyright 2011 American Institute of Physics. 
energy electron diffraction (RHEED) and MOKE measurements were taken across the library, and it was found that the robust ferromagnetism observed with high saturation magnetization (and high Curie temperature) at $\mathrm{Co}_{0.4} \mathrm{Mn}_{0.4} \mathrm{Ge}_{0.2}$ has strong correlation with the onset of a structural orderdisorder transition, revealed in an abrupt change in the RHEED intensity. ${ }^{132}$ Thus, as has been demonstrated in this paper through several examples, high throughput mapping of compositional phase diagrams using various characterization techniques can go beyond simply mapping phase boundaries and allows one to develop important insights concerning the physics of the underlying magnetic phenomena.

Bulk diffusion couples ("multiples") ${ }^{135}$ can also be used to study magnetic properties as a function of composition. Curie temperature-composition relationships can be obtained from bulk diffusion multiples using magnetic force microscopy (MFM), as shown in Fig. 37 for the case of the Co-Cr-Mo ternary system. ${ }^{136}$ Fig. 37(b) shows a SEM backscattered electron image of the Co-Cr-Mo tri-junction area.
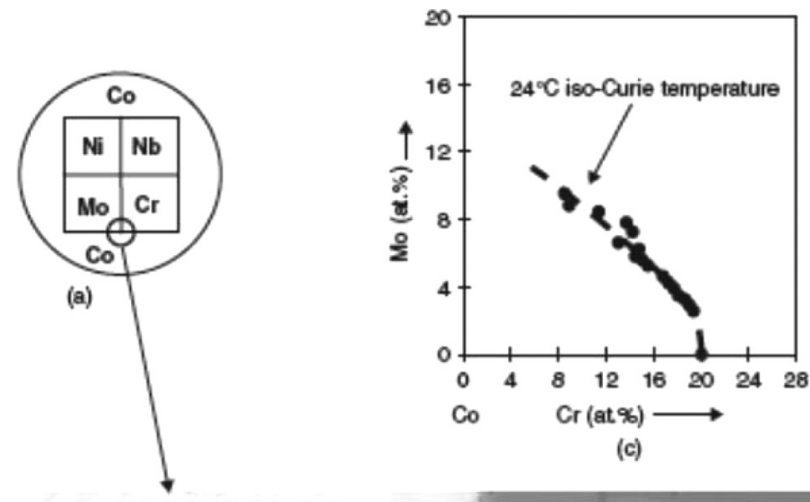

(c)

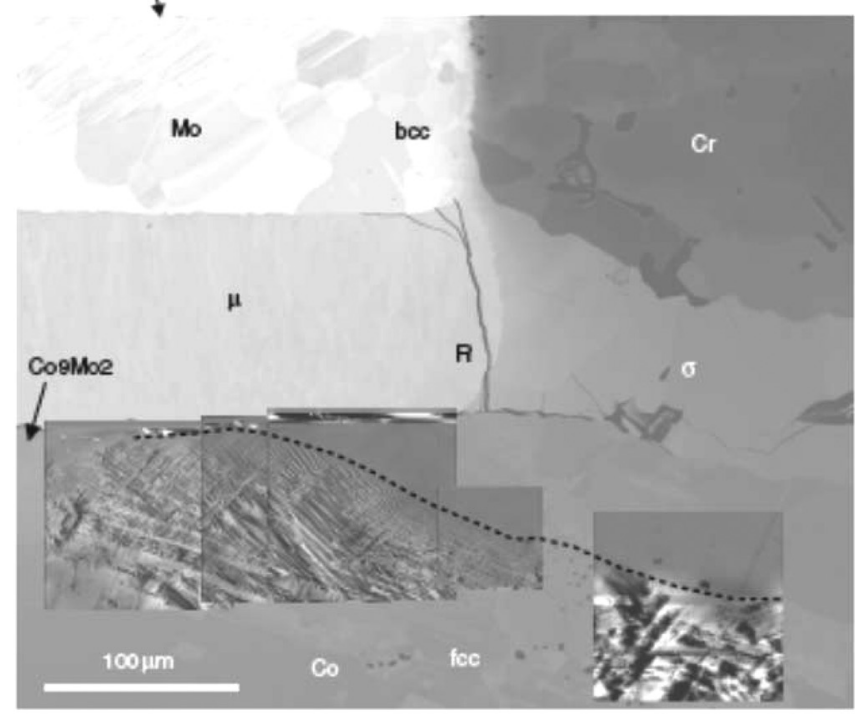

(b)

FIG. 37. Application of MFM to map magnetic domains to obtain Curie temperature-composition relationship for the Co-Cr-Mo ternary system: (a) schematic of a Co-Cr-Mo-Nb-Ni diffusion multiple with the $\mathrm{Co}-\mathrm{Cr}-\mathrm{Mo}$ tri-junction region highlighted; (b) MFM images superimposed on a backscattered electron image of the Co-Cr-Mo tri-junction; and (c) the $24{ }^{\circ} \mathrm{C}$ iso-Curie temperature compositions of the fcc phase in the $\mathrm{Co}-\mathrm{Cr}-\mathrm{Mo}$ ternary system obtained by tracing the boundary (dotted line in (b)) between the domain/non-domain region with EPMA. Reproduced with permission from J. C. Zhao, Prog. Mater. Sci. 51, 557 (2006). Copyright 2006 Pergamon.
Superimposed on the image are MFM maps (taken at $\approx 24^{\circ} \mathrm{C}$ ) displaying magnetic domains in Co rich areas of the fcc phase; topographic features such as polishing scratches and voids allowed the exact positions of the MFM maps to be superimposed on the SEM image. The ferromagnetic region of the fcc phase could be separated from the paramagnetic region, as shown by the dotted line in Fig. 37(b). EPMA along this line revealed the compositions at which the magnetic transition takes place at $24^{\circ} \mathrm{C}$, i.e., an iso-Curie temperature line at $24^{\circ} \mathrm{C}$, Fig. 37(c). By performing MFM mapping at different temperatures, it would have been possible to obtain the constant Curie temperature surface for the fcc phase.

While this review article is mostly focused on thin film based combinatorial techniques, it is important to point out the significance of the diffusion couple (or "multiple") sample techniques. In Sec. III, the utility of a bulk based approach to mapping the properties of piezoelectric ceramics was discussed. The necessity to map the properties of materials in bulk form is particularly acute in a variety of metallic alloy systems, where subtle change in composition at different length scales can dramatically influence the mechanical properties. In addition to magnetic materials, the bulk mapping technique is effective in investigation of structural materials such as superalloys. Diffusion multiple samples can be used to determine phase diagrams and design functional alloys. ${ }^{137-141}$ However, one disadvantage of the bulk diffusion multiple techniques is that property measurements must be carried out with very high spatial resolution of the order of 1 to 5 micrometers, due to the limited scale of diffusion in bulk samples.

The magnetic properties of thin films and multilayers can change dramatically as a function of thickness, because the length scale that determines the exchange interaction of magnetic layers of the order of several nanometers is small. In fact, in the magnetic recording head industry, subnanometer dimensional control of the multilayer thicknesses is necessary for state-of-the-art technology. Because it is relatively straightforward to prepare a thickness gradient film, the socalled "wedge" structure has long been used to investigate the effect of thickness (typically using a single component film) on magnetic properties. This approach was successfully implemented in a $\mathrm{Fe}(1 \mathrm{~nm}) / \mathrm{Cr}(0-15 \mathrm{~nm}) / \mathrm{Fe}$ sandwich/ wedge structure to systematically study the interlayer thickness dependence on exchange coupling between thin films. ${ }^{142,143}$ Figure 38 is a scanning electron microscopy polarization analysis (SEMPA) image of the $\mathrm{Fe}-\mathrm{Cr}-\mathrm{Fe}$ trilayer with varying $\mathrm{Cr}$ thickness, showing the periodic reversal of the sign of the exchange coupling due to the Ruderman-Kittel-Kasuya-Yosida (RKKY) interaction. ${ }^{144,145}$

Given the urgent need for new permanent magnets with higher energy products for next generation motor vehicle and wind power generator applications, high throughput approaches are expected to play an important role in the discovery of new magnetic materials; the need is particularly acute for RE-free permanent magnets with high coercive fields. ${ }^{146,147}$ One proposed approach to synthesizing high energy product permanent magnets is to combine a high coercive field hard magnet with a high magnetization soft 


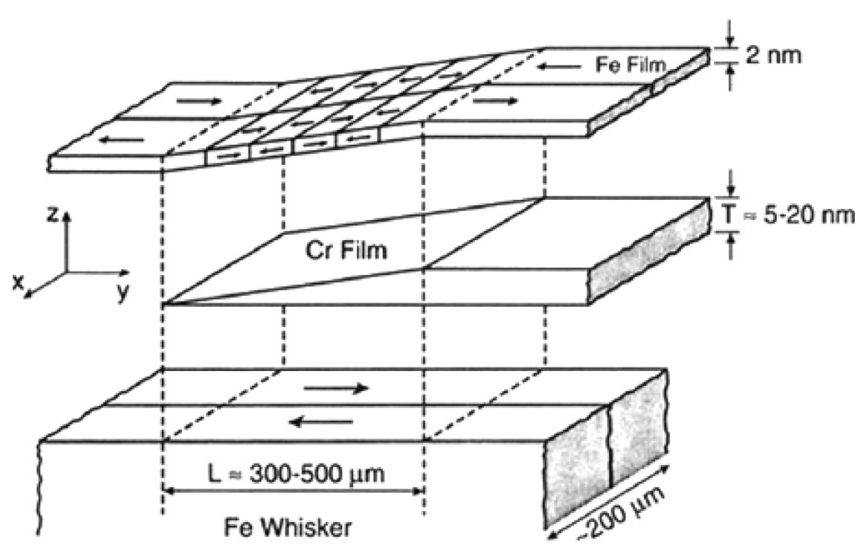

FIG. 38. SEMPA (scanning electron microscopy with polarization analysis) image of a thickness wedge sample, showing the quasiperiodic oscillations of the exchange coupling between two Fe films separated by a $\mathrm{Cr}$ film of $0-15 \mathrm{~nm}$. The light and dark contrast represents regions in which the magnetization of the top Fe layer is aligned or antialigned with that of the bottom layer. Reproduced with permission from J. Unguris et al., Phys. Rev. Lett. 67, 140 (1991). Copyright 1991 American Physical Society.

magnet in a tightly coupled nanocomposite configuration, so that the overall material displays the combination of high coercive field and high magnetization. ${ }^{148}$ Since exchange pinning of the soft layer by the hard magnet is the key to achieving this effect, it is critical to understand the mechanisms and parameters that enable optimum coupling. To this end, thin film multilayers have been used as a model system to understand the micromagnetic parameters that determine the coupling behavior. ${ }^{149,150}$ The gradient thickness approach has also been used to investigate the thickness of a high magnetization Fe layer coupled to a high coercivity
SmCo layer using X-ray magnetic circular dichroism (XMCD) to determine the critical soft layer thickness pinned by $\mathrm{SmCo} .{ }^{103}$ Such research has further been extended to investigate the relationship between soft phase micromagnetic constants and thickness in an $\mathrm{Fe}-\mathrm{Co} / \mathrm{CoPt}$ bilayer square library deposited by e-beam evaporation. Using automated shadow masks, the thickness of the soft phase was varied from 1 to $10 \mathrm{~nm}$ in one direction, and the composition of the soft phase, $\mathrm{Fe}_{\mathrm{x}} \mathrm{Co}_{1-\mathrm{x}}$, in the other, ${ }^{151}$ Fig. 39(a). Figure 39(b) shows the MOKE hysteresis loops taken at each position on the library. This plot can be used to visually determine the effect of the soft phase composition on the exchange coupling length, Fig. 39(c), i.e., the length beyond which a region of the soft phase is decoupled from the hard layer. This study revealed that the most important parameters determining the exchange coupling length are the hard phase coercive field and the soft phase magnetization.

Both ferromagnetic SMA and magnetostrictive materials possess magnetoelastic properties, i.e., in response to an applied magnetic field, a strain is induced in the material, and vice versa. Magnetoelastic materials are a technologically important class of materials with applications in sensors and actuators. Among magnetoelastic materials which display Joule magnetostriction, Tb-Dy-Fe (terfenol) exhibits the largest field induced strain, with saturation magnetostriction of more than $1000 \mathrm{ppm}(0.1 \%$ strain). With the advent of novel remote sensing technologies and the ubiquity of smart microactuators in microsystems, there is a need to find inexpensive, non-brittle, RE-free substitutes for terfenol. Recently, $\mathrm{Fe}_{1-\mathrm{x}} \mathrm{Ga}_{\mathrm{x}}$ was found to display a significant magnetostriction of $3 / 2\left(\lambda_{100}\right)>400 \mathrm{ppm}$. Subsequently, it was

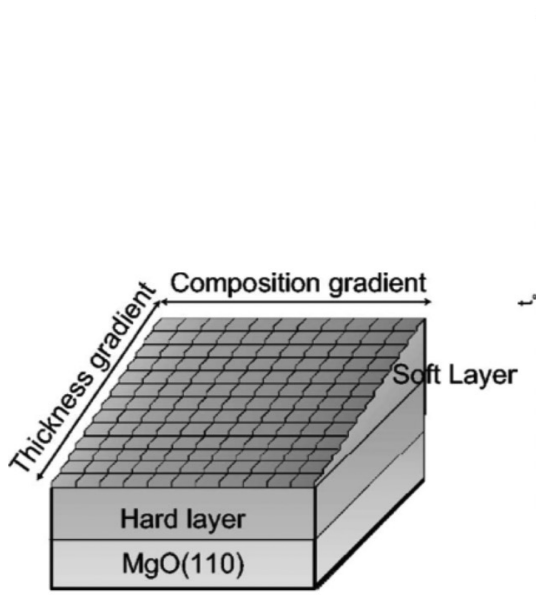

(a)

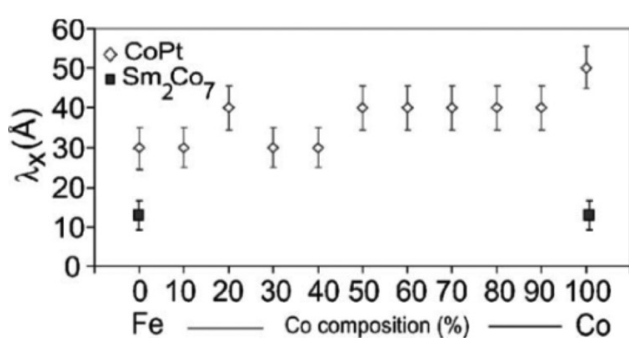

(c)

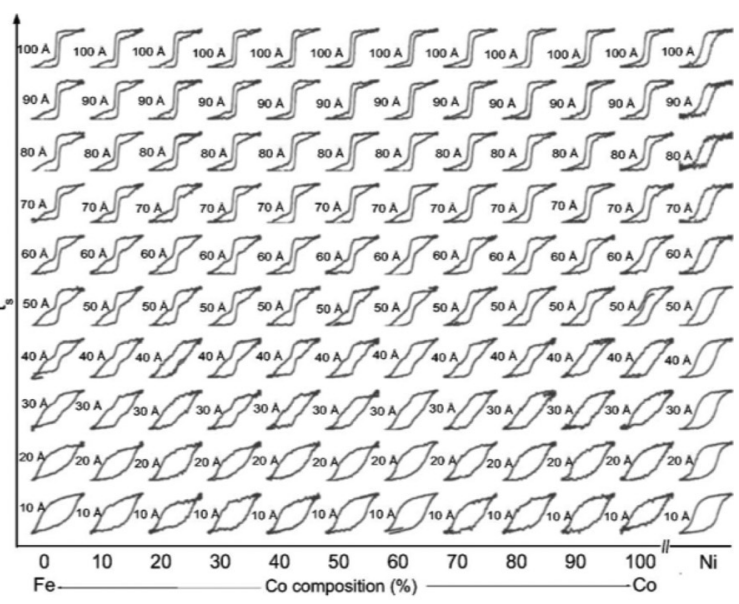

(b)

FIG. 39. (a) Schematic of the library for investigating the coupling behavior of hard/soft bilayers (left), (b) magnetic hysteresis loops (a.u.) for different soft layer thicknesses $\left(\mathrm{t}_{\mathrm{s}}\right)$ and compositions on a $\mathrm{CoPt}\left(\mathrm{H}_{\mathrm{c}}=0.64 \mathrm{~T}\right)$ hard layer measured on a single library, and (c) the coupling length $\lambda_{\mathrm{x}}$, experimentally determined from the soft layer thickness at which the transition between one phase-like behavior and two-phase-like behavior takes place. Reproduced with permission from A. J. Zambano et al., Phys. Rev. B 75, 144429 (2007). Copyright 2007 American Physical Society. 
found that structural heterogeneity at a compositional phase boundary can lead to formation of a phase, which exhibits substantial magnetostriction. ${ }^{152}$ A thin film cantilever method to map the magnetostrictive properties of cosputtered $\mathrm{Fe}-\mathrm{Ga}$ and $\mathrm{Fe}-\mathrm{Ga}-\mathrm{Al} \mathrm{CCS}$ libraries was devised ${ }^{153}$ in which a laser beam reflected from the tip of the cantilever was used to determine its deflection as a function of applied magnetic field. The measured deflection was then used to determine the magnetostrictive coefficient. This work showed that the observed bulk magnetostriction trend with composition could be reproduced in thin films, Fig. 40. In another Fe-Ga-Al CCS study, ${ }^{117}$ Mossbauer spectroscopy was used to understand the effects of $\mathrm{Ga}$ and $\mathrm{Al}$ additions to the magnetic environment of bcc $\mathrm{Fe}$; $\mathrm{Ga}$ atom pairing is believed to be the reason for magnetostriction enhancement, and this is directly affected by Al substitution. In a related magnetostriction experiment, ${ }^{154}$ it was found that upon annealing and quenching Co-Fe films, a large magnetostriction was observed at compositions at the $\mathrm{bcc} /(\mathrm{fcc}+\mathrm{bcc})$ phase boundary. This study underscores the role structural heterogeneity plays in magnetostrictive properties and the importance of properly controlling the thermal treatment process of thin film libraries to obtain accurate property trends as a function of composition. ${ }^{155}$

\section{Superconductors}

Superconductivity remains one of the most intensively studied fields, and superconducting materials are a constant target for materials exploration. Superconducting materials are used in a wide variety of advanced technology applications ranging from ultrasensitive magnetometers and ultrafast logic circuits to superconducting power cables and magnetic levitation. In the mid-1980s, the discovery ${ }^{156}$ of high temperature superconductivity in cuprates led to a revolution in the field.

A chemical precursor based thin film synthesis method to fabricate libraries of cuprate superconductors has been developed. ${ }^{8}$ This work was the first demonstration of thin

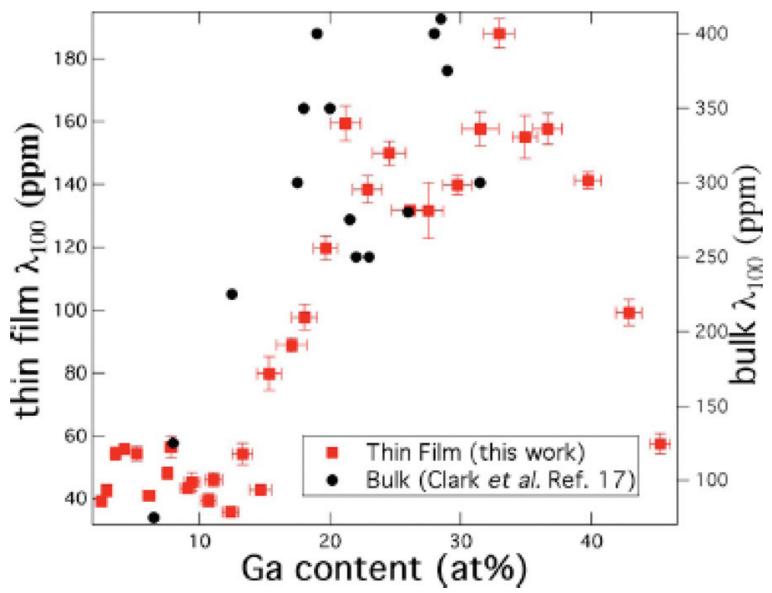

FIG. 40. Magnetostrictive coefficient measured from Fe-Ga thin-film composition spread plotted against values from bulk studies as a function of $\mathrm{Ga}$ content. The compositional trend agrees well with bulk values. Reprinted with permission from J. R. Hattrick-Simpers et al., Appl. Phys. Lett. 93(10), 102507 (2008). Copyright 2008 American Institute of Physics.

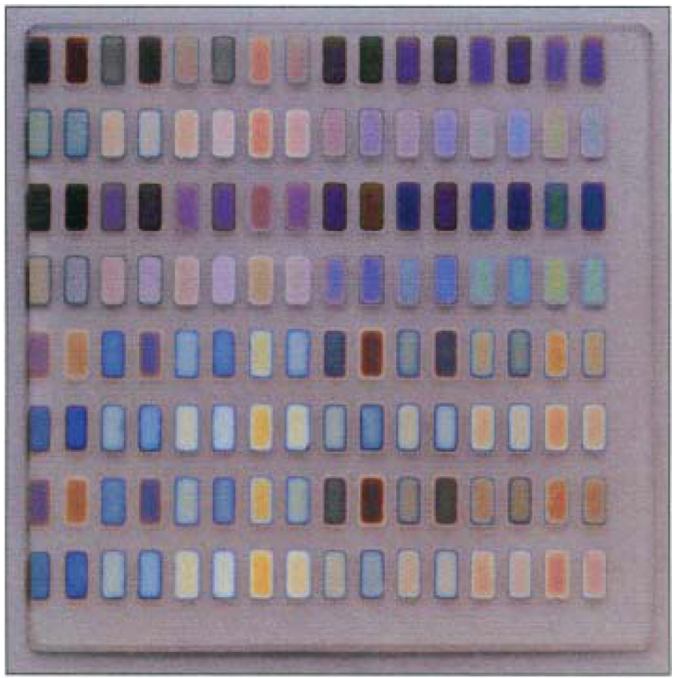

FIG. 41. A 128-member matrix array cuprate superconductor library, prior to sintering. Each site is $1 \mathrm{~mm}$ by $2 \mathrm{~mm}$; the color of each is the natural color of reflected light from a white light source. The amorphous precursor layers are deposited using a series of shadow masks. Reproduced with permission from X. D. Xiang et al., Science 268, 1738 (1995). Copyright 1995 American Association for the Advancement of Science.

film libraries of inorganic functional materials in a discrete composition matrix array, Fig. 41. Different combinations of amorphous precursor layers were deposited using a series of shadow masks. Screening of this array was performed using a multichannel four terminal resistance measurement system, and many of the matrix elements (consisting of up to 7 elements) were correctly identified as exhibiting superconducting transitions from resistance-temperature measurements. This work not only demonstrated the utility of the combinatorial synthesis of libraries of functional inorganic materials but also showed that the thin film amorphous precursor technique could be used to synthesize multi-element compositions with complex crystal structures.

Several groups have adopted the parallel four-terminal resistance-temperature measurement method for rapid screening of new superconductors. ${ }^{157,158}$ Figure 42 shows a 196 pin probe array, which allows simultaneous resistance

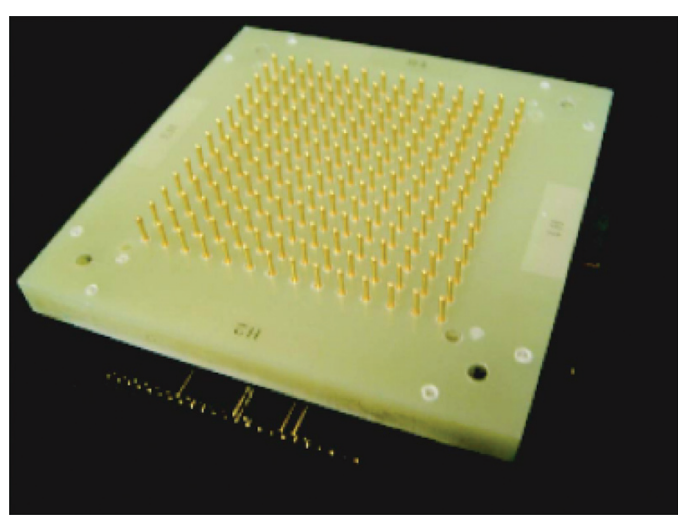

FIG. 42. The 196 pin layout that makes electrical contact to a 49 sample thin film library for simultaneous four terminal resistivity measurements. The shortest distance between pins is $4.64 \mathrm{~mm}$. Reprinted with permission from K. C. Hewitt et al., Rev. Sci. Instrum. 76, 093906 (2005). Copyright 2005 American Institute of Physics. 
measurements of up to 49 samples in a liquid He cryostat. Another detection technique that has been applied for mapping superconducting materials is magnetic field modulated microwave absorption, ${ }^{159,160}$ which takes advantage of the fact that in the presence of a modulating magnetic field, superconducting regions of a material absorb microwave power due to flux penetration. A common screening approach is to apply this probe to a thin film library. ${ }^{159-163}$

Despite decades of international research, the mechanisms of superconductivity in many compounds are not fully understood. The recent discovery of high temperature superconductivity in Fe pnictides ${ }^{164}$ has reignited the search for new superconductors, and high throughput methodologies will continue to play an important role. Mapping of phase diagrams can be an important part of deciphering the physics of superconducting mechanisms. As an example, the use of combinatorial techniques to map phase space in the Ba-Y$\mathrm{Cu}-\mathrm{O}$ system can be found in the work of Wong-Ng et al. ${ }^{165}$

\section{OPTICAL MATERIALS}

\section{A. Thermochromics and electrochromics}

It has been estimated that $20 \%-30 \%$ of the energy used to heat and cool buildings in the US is lost through windows, due to undesired solar heating and thermal conduction. ${ }^{166}$ "Smart" windows that have either thermochromic coatings (i.e., films whose solar acceptance varies as a function of temperature), or electrochromic coatings (i.e., films whose solar acceptance varies as a function of applied bias) have great potential to minimize building energy losses. These coatings are competing technologies for the "smart" component of windows; electrochromic windows require a power supply, but are easier to adjust than thermochromic windows, which are simply sensitive to temperature.

A novel screening methodology for high throughput studies of electro-optically active materials has been designed using an optical screen containing an electrochromic film of $\mathrm{WO}_{3}{ }^{167}$ However, electrochromic materials for window applications have not been studied combinatorially. Thermochromic materials that undergo a reversible phase transformation close to RT are attractive options for smart window coatings; the low temperature phase of lightly doped $\mathrm{VO}_{2}$ is transparent to IR radiation, thus allowing natural heating, while the higher temperature phase reflects the IR, thus limiting excess heating of the building when the external temperature is high. Both phases are transparent to the visible spectrum; thus, the window's clarity and esthetic appeal are maintained. Ideally, phase transition temperatures $\left(\mathrm{T}_{\mathrm{c}}\right)$ for thermochromic window coatings would be in the ambient range, i.e., $-20^{\circ} \mathrm{C}$ to $35^{\circ} \mathrm{C}$. At $68^{\circ} \mathrm{C}, \mathrm{VO}_{2}$ has the closest $\mathrm{T}_{\mathrm{c}}$ of the unary metal oxides. $\mathrm{W}, \mathrm{Mo}, \mathrm{Ti}, \mathrm{Nb}$, and $\mathrm{Fe}$ dopants can depress $\mathrm{T}_{\mathrm{c}}$ at rates of $10^{\circ} \mathrm{C}-25^{\circ} \mathrm{C} / \mathrm{at} . \%{ }^{168-170}$ and should be studied combinatorially.

Oxygen partial pressure has been varied combinatorially to study its effect on $\mathrm{VO}_{\mathrm{x}}$ stoichiometry in PLD films. ${ }^{171}$ The processing window for preparing a $\mathrm{VO}_{2}$ film from either a $\mathrm{V}_{2} \mathrm{O}_{3}$ or $\mathrm{V}_{2} \mathrm{O}_{5}$ target is shown in Fig. 43. Thin $\mathrm{VO}_{2}$ thermochromic films have also been deposited by an electrostatic atomization technique. ${ }^{172}$ Although the paper reports the (a)
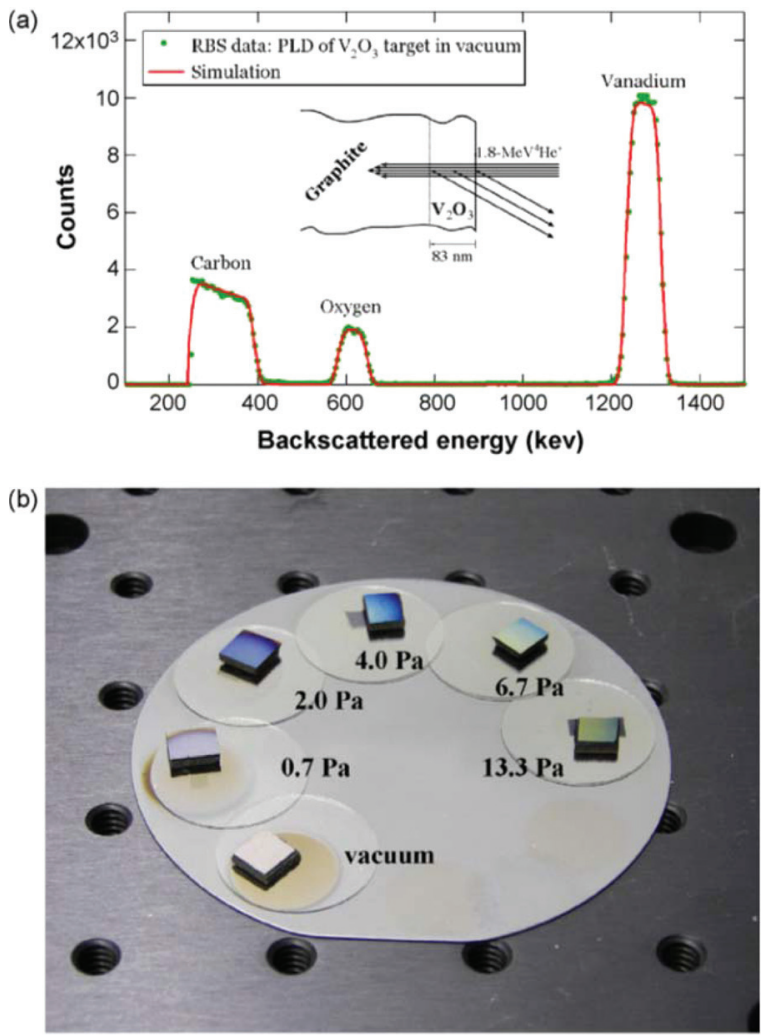

(c)

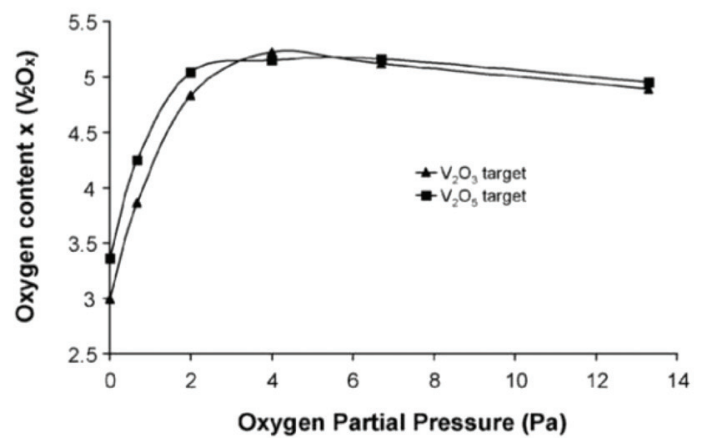

FIG. 43. (a) Experimental and simulated RBS spectra for a $\mathrm{V}_{2} \mathrm{O}_{\mathrm{x}}$ film (from $\mathrm{V}_{2} \mathrm{O}_{3}$ target in vacuum): $\mathrm{x}=2.99$, thickness $=83 \mathrm{~nm}$; (inset) RBS schematic, (b) photograph of $\mathrm{V}_{2} \mathrm{O}_{\mathrm{x}}$ library with indicated values of oxygen partial pressure during deposition, and (c) oxygen stoichiometry in the library as a function of background pressure of oxygen. Reproduced with permission from N. D. Bassim et al., Appl. Surf. Sci. 254, 785 (2007). Copyright 2007 Elsevier BV.

deposition of film "libraries," it is not clear what parameter was varied in the preparation of these libraries.

\section{B. Luminescents and phosphorescents}

Luminescent materials emit visible radiation by a variety of mechanisms, such as electrical stimulation, absorption of photons, or a chemical reaction, but not by heat (emission due to heat is called incandescence). A subset of luminescent materials, phosphorescents, slowly (scale of minutes to hours) emits visible radiation after absorption of photons. Many early combinatorial thin film experiments ${ }^{173-178}$ were designed to explore novel luminescent materials, because such libraries served as excellent demonstrations of the efficacy of the combinatorial approach; initial high-throughput screening is very simple, as one only had to view optically 


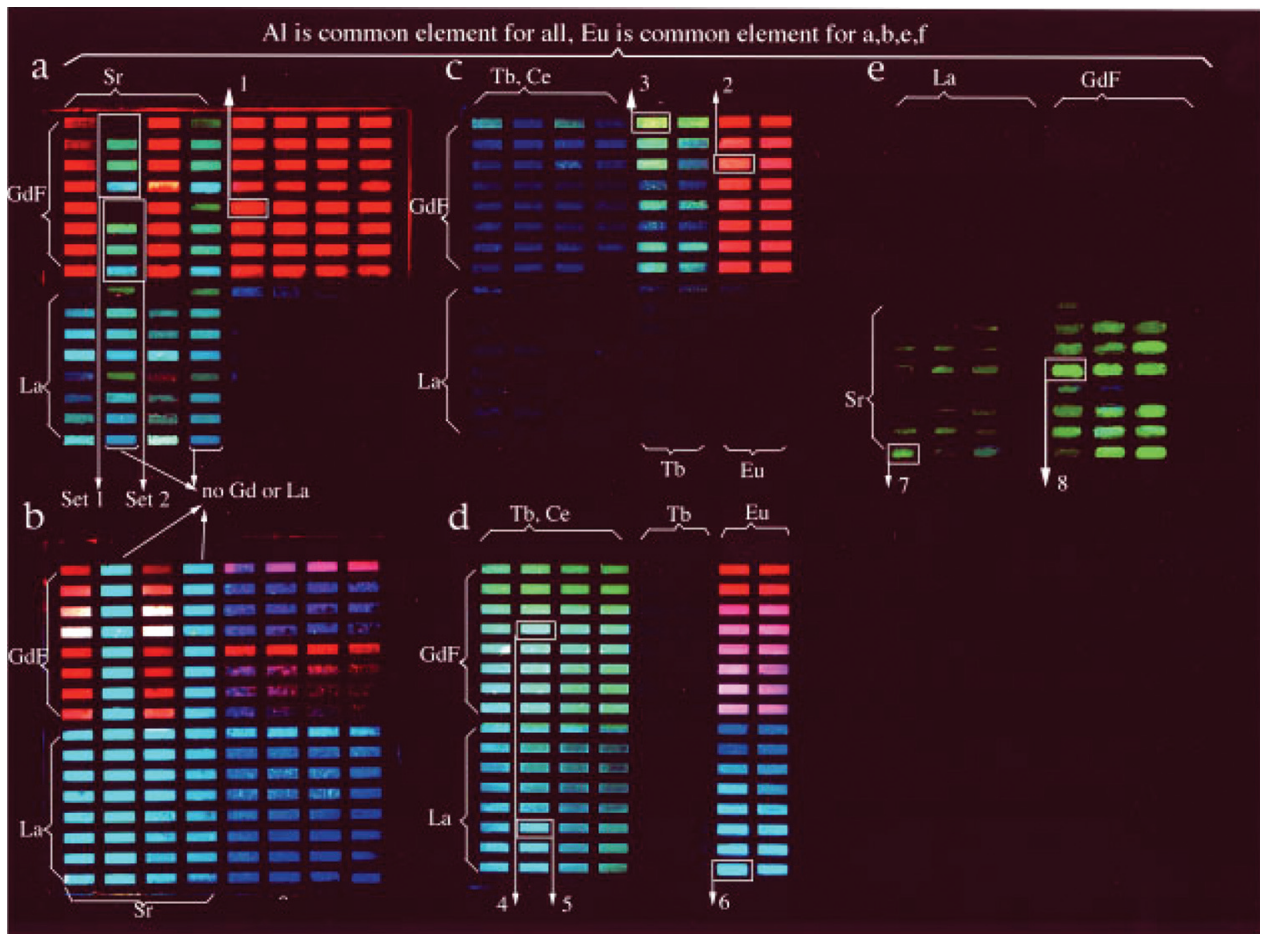

FIG. 44. Photoluminescence images of a series of phosphor libraries processed under different conditions with nominal compositions indicated. (a) $\mathrm{La}\left(\text { or } \mathrm{GdF}_{3}\right)_{\mathrm{m}}$ $(\mathrm{Sr})_{\mathrm{n}} \mathrm{AlO}_{\mathrm{x}}: \mathrm{Eu}_{\mathrm{y}}{ }^{2+/ 3+}$ where $0.375 \leq \mathrm{m} \leq 1$, $0.25 \leq \mathrm{n} \leq 0.4,1.88 \leq \mathrm{y} \leq 12 \%$ in atomic ratio, annealed at $1150^{\circ} \mathrm{C}$; (b) same as (a) but annealed at $1400^{\circ} \mathrm{C}$; (c) $\mathrm{La}$ (or $\left.\mathrm{GdF}_{3}\right)_{\mathrm{m}} \mathrm{AlO}_{\mathrm{x}}: \mathrm{Tb}_{\mathrm{y}}{ }^{3+}\left(\mathrm{Ce}_{\mathrm{z}}{ }^{3+}\right) ; \quad \mathrm{Eu}_{\mathrm{h}}{ }^{2+/ 3+}$ where $\quad 0.32 \leq \mathrm{m} \leq 1, \quad 1.29 \leq \mathrm{y} \leq 6$, $0.65 \leq \mathrm{z} \leq 4 \%, 1.29 \leq \mathrm{h} \leq 8 \%$ annealed at $1150^{\circ} \mathrm{C}$; (d) same as (c), but annealed at $1400^{\circ} \mathrm{C} ; \quad$ (e) $\mathrm{La}\left(\text { or } \quad \mathrm{GdF}_{3}\right)_{\mathrm{m}}(\mathrm{Sr})_{\mathrm{n}}$ $\mathrm{AlO}_{\mathrm{x}}: \mathrm{Eu}_{\mathrm{y}}{ }^{2+/ 3+}$ where $0.178 \leq \mathrm{m} \leq 0.714$, $0.17 \leq \mathrm{n} \leq 0.4,0.75 \leq \mathrm{y} \leq 16.7 \%$ annealed at $1150^{\circ} \mathrm{C}$. Each substrate is $1 \mathrm{in}$. square. The images are taken with UV lamp excitation. Reprinted with permission from X. D. Sun et al., Appl. Phys. Lett. 70, 3353 (1997). Copyright 1997 American Institute of Physics.

excited libraries and visually detect, which composition sites were emitting light.

Phosphorescent materials are essential for lighting, imaging, and display applications including plasma, field emission, electroluminescent, and cathode ray tube (CRT) projection displays. Although the photophysical processes leading to luminescence are relatively well understood, their specific spectral properties, luminescence efficiencies, and operational lifetimes depend on a complex interplay between the excitation source, host crystal lattice, sensitizer, and luminescent centers. Identification of phosphors that satisfy the set of requirements of a given display application can be highly empirical, and therefore combinatorial methodologies can play a significant role in optimizing their compositions. Beyond initial screening, it is important to carry out quantitative characterization of luminescent materials libraries using specific metrics, such as emission spectrum, chromaticity (a measure of the quality of a color based on hue and dominant wavelength), and quantum efficiency.

Sun et al. ${ }^{179}$ were the first to show the utility of the combinatorial approach for luminescent materials. Using precursor layers deposited by RF sputtering through a set of physical masks, they fabricated thin film libraries to investigate $\mathrm{RE}$ activated refractory metal oxides, $\mathrm{Gd}(\mathrm{La}, \mathrm{Sr}) \mathrm{AlO}_{\mathrm{x}}$. Their findings are summarized in Fig. 44; from this study, a green emitting composition, $\mathrm{Gd}_{0.46} \mathrm{Sr}_{0.31} \mathrm{Al}_{1.23} \mathrm{O}_{\mathrm{x}} \mathrm{F}_{1.38}: \mathrm{Eu}_{0.06}$ was identified that had better chromaticity (as well as a high quantum efficiency, $>94 \%$ ), than that of the standard $\mathrm{Y}_{3}(\mathrm{Al}, \mathrm{Ga})_{5} \mathrm{O}_{12}: \mathrm{Tb}$. The same library has been characterized by synchrotron micro-x-ray beam experiments, where structural properties, composition distribution, and dopant ionization states were mapped in a single experiment. ${ }^{180}$ In a related study, a red phosphor with a quantum efficiency of $86 \%$ was found in the $\left(\mathrm{Gd}_{2-\mathrm{x}} \mathrm{Zn}_{\mathrm{x}}\right) \mathrm{O}_{3-\delta}: \mathrm{Eu}^{3+}$ system. ${ }^{173}$ This phosphor has a better chromaticity than the state of the art red phosphor, $\mathrm{Y}_{2} \mathrm{O}_{3}: \mathrm{Eu}^{3+}$, and it exhibits enhanced color saturation.

A segmented masking scheme, combined with the use of precursor layers, has proven to be particularly effective for designing phosphor composition libraries. In one study, a quaternary scheme was implemented using photolithography for investigation of a large number of different host materials, combined with dopant activation elements. ${ }^{174}$ Figure 45 shows the set of quaternary masks used to fabricate a 1024 element quaternary library. In this scheme, each mask is used four times, each time rotated by $90^{\circ}$, to segment particular compositional variations into four distinct groups. Thus, using the five masks, compositions of the type $\mathrm{ABCDE}$ can be mapped, where each letter A, B, C, D, or E represents a four-fold doping element variation, leading to 1024 distinct combinations. ${ }^{174}$ In this doping experiment, the mask pattern

\section{Quaternary \\ Masks}

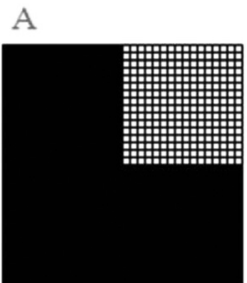

D

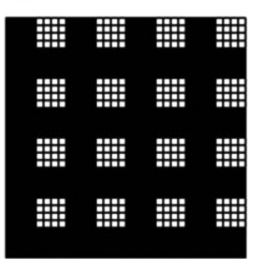

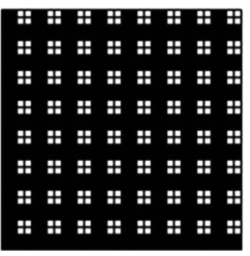

B

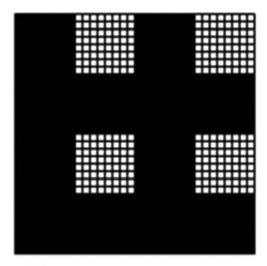

E

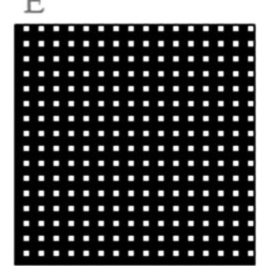

FIG. 45. Masks for generating a quaternary library. Reproduced with permission from J. S. Wang et al., Science 279, 1712 (1998). Copyright 1998 American Association for the Advancement of Science. 
was created using a photolithographic lift-off technique, and deposition of the precursor materials was by PLD, followed by a series of thermal treatments to interdiffuse the precursor layers and crystallize the compounds. A scanning spectrometer was used for quantitative screening of the library; a well-characterized phosphor, $\mathrm{Zn}_{2} \mathrm{SiO}_{4}$ : Mn, was used as a reference standard. In this study, a blue luminescent material always sought by the display manufacturing community, $\mathrm{Gd}_{3} \mathrm{Ga}_{5} \mathrm{O}_{12} / \mathrm{SiO}_{2}$, was discovered combinatorially.

A variety of other techniques have been implemented for synthesizing libraries of luminescent materials, including cosputtered $\operatorname{CCS}^{181,182}$ and a solution-based technique using a multihead scanning ink-jet delivery system, ${ }^{175}$ Fig. 46. Such inkjet delivery systems are broadly applicable for combinatorial synthesis of other materials systems. A key advantage of the inkjet delivery system is that precursors can be mixed at the molecular level in the nozzle prior to controlled delivery at each site, thus avoiding the need for thermal interdiffusion treatment of the library. ${ }^{183}$ In another study, ${ }^{176}$ electron beam deposition in a moving shutter system was used to create a large library containing composition gradients of multiple phosphor hosts as well as different RE dopants, Fig. 47. This work led to the discovery of a new RE phosphor, $\mathrm{Sr}_{2} \mathrm{CeO}_{4}$, containing one-dimensional chains of $\mathrm{CeO}_{6}$ octahedra in their crystal structure. A more recent combinatorial experiment on $\mathrm{ZnO}$-based phosphors ${ }^{184}$ involved lattice engineering via precise, unit cell deposition enabled by automated mask motion. The unit cell engineered deposition method results in an extremely well controlled library, and as a result, luminescent behavior can be attributed to

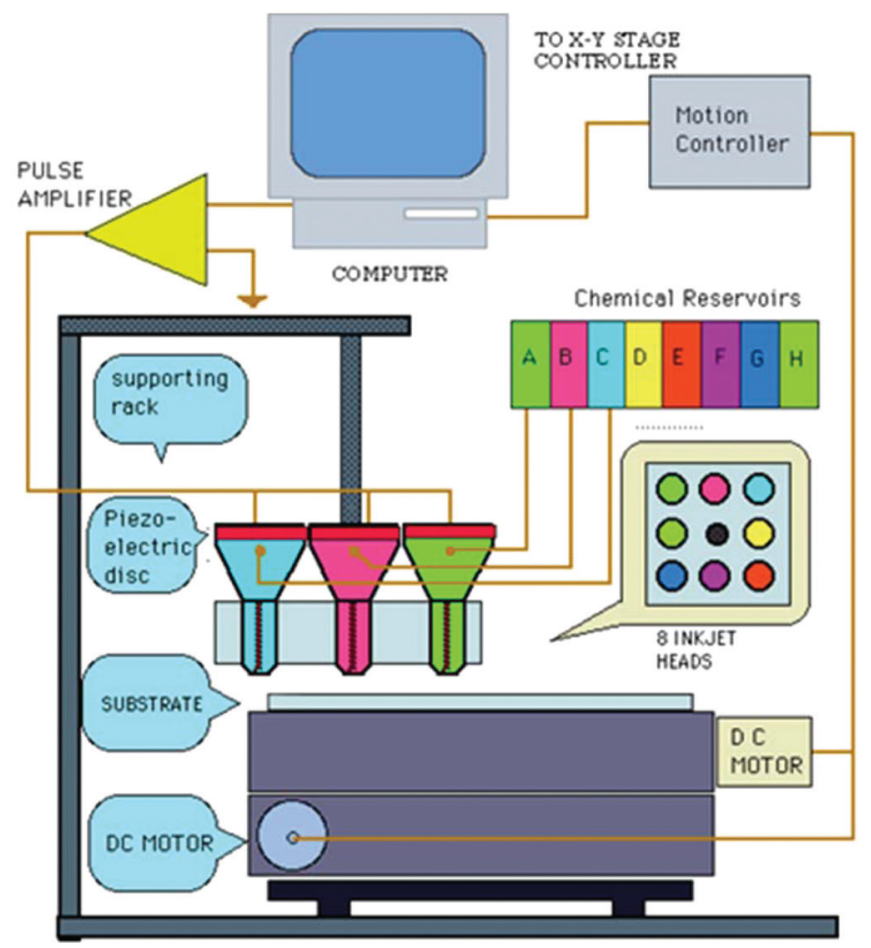

FIG. 46. Schematic of a scanning multihead inkjet delivery system for solution deposition of optical material libraries. The system is integrated from drop-on-demand single nozzle piezoelectric inkjets (see Ref. 435). Reproduced with permission from T. X. Sun, in Combinatorial Materials Synthesis, edited by X. D. Xiang and I. Takeuchi (Marcel Dekker, New York, 2003), pp. 141-176.

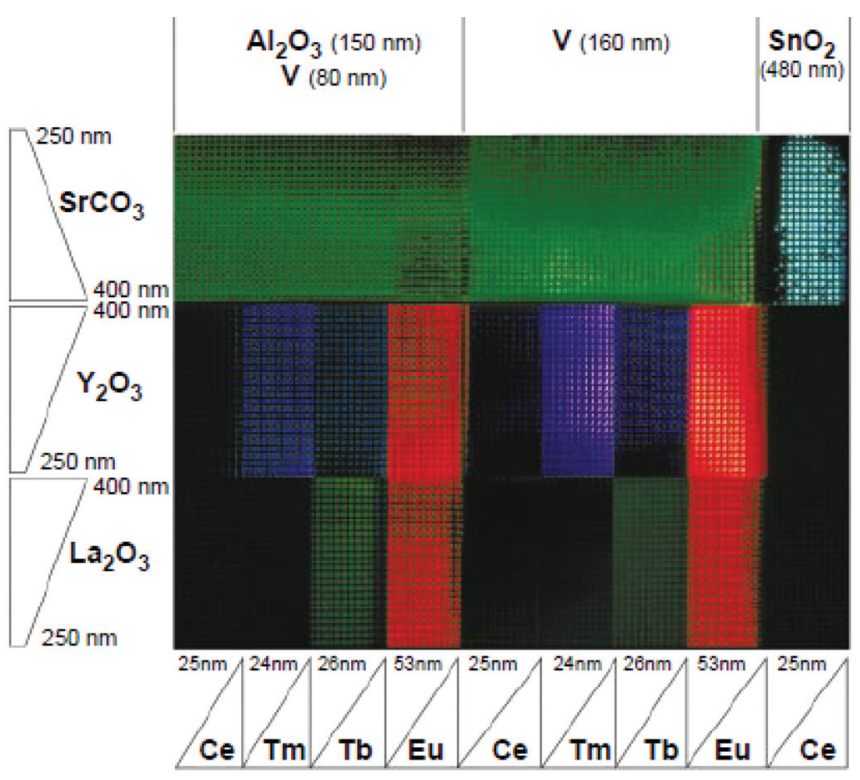

FIG. 47. Image of UV excited $(254 \mathrm{~nm})$ photoluminescence from a library. The blue-white emission in the upper right corner is from $\mathrm{Sr}_{2} \mathrm{CeO}_{4}$. Reproduced with permission from E. Danielson et al., Science 279, 837 (1998). Copyright 1998 American Association for the Advancement of Science.

precise positions on the library. A series of new phosphors including blue ( $\mathrm{ZnO}: \mathrm{W}, \mathrm{Mg}$, and $\mathrm{ZnO}: \mathrm{W})$, red $(\mathrm{ZnO}: \mathrm{Y}, \mathrm{Eu})$, and yellow $(\mathrm{ZnO}: \mathrm{V})$ have been identified from this study. Ink-jet printing has also been successfully used to screen and optimize polymeric materials for organic light-emitting diodes and other optoelectronic device applications. ${ }^{185-187}$

$\mathrm{ZnO}$ is a versatile wide bandgap semiconductor material; to date, a variety of devices including light emitting pnjunctions, lasers, gas sensors, and photodetectors have been demonstrated using $\mathrm{ZnO}$ and related compounds. ${ }^{188}$ The combinatorial approach has proven to be an effective way to help expand the utility of this material. By controlling the number of pulses used to ablate $\mathrm{ZnO}$ and $\mathrm{Zn}_{0.9} \mathrm{Mg}_{0.1} \mathrm{O}$ targets, bandgap engineering has been demonstrated in an epitaxial library of $\mathrm{Zn}_{1-\mathrm{x}} \mathrm{Mg}_{\mathrm{x}} \mathrm{O}$ thin films. ${ }^{189}$ Fig. 48 shows the deposition cycle scheme, which was used to create a discrete library consisting of nine different members with different $\mathrm{Mg}$ substitution concentrations. In another study, a CCS library spanning the entire $\mathrm{Zn}_{1-\mathrm{x}} \mathrm{Mg}_{\mathrm{x}} \mathrm{O}$ binary system was fabricated using a layer-by-layer epitaxial deposition approach. ${ }^{190,191}$ Figure 49 shows the continuous $\theta-2 \theta$ XRD plot taken across the entire composition spread, showing the evolution of structural phases. From this study, complete bandgap mapping of the $\mathrm{Zn}_{1-\mathrm{x}} \mathrm{Mg}_{\mathrm{x}} \mathrm{O}$ was carried out, and the presence of a mixed phase region in the middle of the composition region was revealed. The same CCS library was used to demonstrate ultraviolet photodetector arrays, where each device in the array has a slightly different peak response wavelength due to the continuously changing bandgap.

\section{Transparent conducting oxides (for photovoltaics)}

Transparent conducting oxides (TCOs) enable a variety of important applications including photovoltaic (PV) cells, touch screens, flat panel displays, and light emitting diodes. 


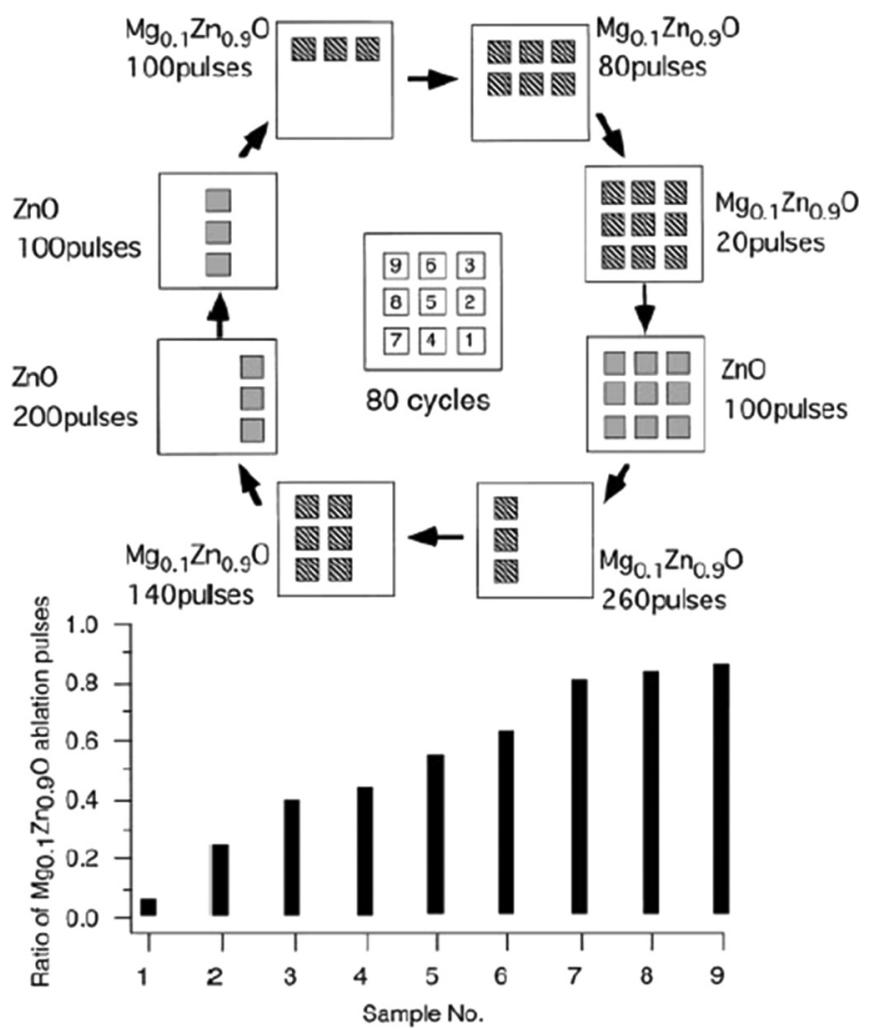

FIG. 48. Schematic diagram of a combinatorial laser molecular beam epitaxy deposition system. Reproduced with permission from Y. Matsumoto et al., Jpn. J. Appl. Phys., Part 2 38, L603 (1999). Copyright 1999 The Japan Society for Applied Physics.

Their primary function in photovoltaics is as a transparent electrode, either at the front or back of the cell, for collection of photogenerated charge carriers. Therefore, the most important properties of a TCO are low resistivity $\left(<5 \times 10^{-4}\right.$ $\Omega$-cm, or high conductivity, $\left.2000(\Omega-\mathrm{cm})^{-1}\right)$ and high transparency in the solar spectrum range $(>80 \%$ from $300 \mathrm{~nm}$ to $1200 \mathrm{~nm}$ wavelengths). Secondary desired properties include thermal, chemical and interfacial stability, high carrier

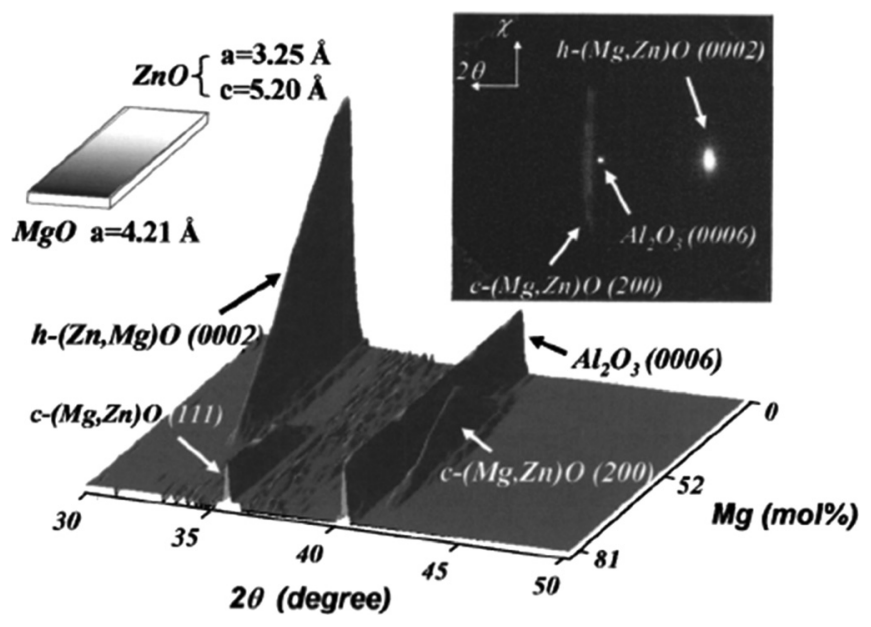

FIG. 49. X-ray diffraction of a $\mathrm{Mg}_{\mathrm{x}} \mathrm{Zn}_{1-\mathrm{x}} \mathrm{O}$ composition spread in the $2 \theta$ range of $30^{\circ}$ to $50^{\circ}$. The vertical scale is arbitrary, but shows the relative intensity of the peak at each $2 \theta$ value at a fixed composition. The upper left shows the schematic of the spread with the lattice constants of the end compositions. Reprinted with permission from I. Takeuchi et al., J. Appl. Phys. 95, 3840 (2004). Copyright 2004 American Institute of Physics. mobility, work function matching with desired photovoltaic materials, and the ability to grow textured films; ${ }^{192}$ further TCO selection criteria are based on price, availability, and material toxicity. Given the large number of elements that may form transparent oxides, and the sensitivity of the final material properties to the presence of dopants as well as processing, the search for optimal TCOs encompasses a large multidimensional parameter space, ideal for high throughput experimentation. Since the first combinatorial experiments on TCOs, ${ }^{193}$ much subsequent research has been devoted to the search for new or improved materials. The majority of the work to date has been focused on the $\mathrm{In}_{2} \mathrm{O}_{3}$ (TIO), $\mathrm{ZnO}$, and $\mathrm{SnO}$ systems. Although this review focuses primarily on TCOs for photovoltaic applications, there has been recent interest in searching for new absorber materials using highthroughput density functional theory (DFT) methods ${ }^{194}$ and combined high-throughput DFT and experimental determinations of novel hole transport layers. ${ }^{195,196}$

\section{1. $\ln _{2} \mathrm{O}_{3}$-based TCOs}

$\mathrm{In}_{2} \mathrm{O}_{3}$-based TCOs are used widely in display applications and as heterojunctions with intrinsic layer photovoltaic cells. Their widespread usage is a result of their high transparency and high conductivity $\left(3-10 \times 10^{3}(\Omega-\mathrm{cm})^{-1}\right)$ when in the crystalline state. However, indium (In) is a costly raw material, and is difficult to dope; a systematic method for doping $\mathrm{In}_{2} \mathrm{O}_{3}$ that preserves or enhances its properties has been pursued for more than $10 \mathrm{yr}$. Tin (Sn) doped TIO has been explored extensively by traditional materials science approaches. Recently, using libraries prepared by liquid titration followed by high temperature annealing, Sn doping of $\mathrm{In}_{2} \mathrm{O}_{3}$ to form highly crystalline films was reported. ${ }^{197}$ The highest transmission reported in this study was $56.4 \%$ at $550 \mathrm{~nm}$, substantially lower than values reported elsewhere.

Extensive research has been performed in alloying $\mathrm{In}_{2} \mathrm{O}_{3}$ with zinc ( $\mathrm{Zn})$ and $\mathrm{Sn}$ to form zinc indium oxide (ZIO) and TIO, which are amorphous TCO materials. Although they typically exhibit lower conductivities than crystalline materials, amorphous films are of interest because they do not require annealing at high temperature. In a comprehensive body of work, ${ }^{193,198-200}$ CCS libraries were deposited in a specially designed reactive sputtering chamber, using an off axis sputtering configuration. Multiple depositions were necessary to fully cover the compositional phase diagram. Library characterization was accomplished with a suite of high throughput tools including EMPA, UV/visible (VIS)/ near infrared (NIR) and Fourier transform infrared spectroscopy (FTIR) measurements, Hall probe, and micro-XRD. In the ZIO system, a broad maximum in conductivity was found to exist at $\mathrm{Zn}$ concentrations between 45 and 80 at. $\% .{ }^{201}$ The conductivity maximum was found by Hall measurements to be $\sim 3000(\Omega-\mathrm{cm})^{-1}$ for In concentrations of $\sim 67 \%$, Fig. 50 . The region of enhanced film conductivity roughly correlates with the amorphous region. All films exhibited optical transmissivities of $>80 \%$ in the visible region. It was also found that a simple Drude approximation could be used to correlate measurements of the plasma wavelength to the electron carrier concentration variations. 


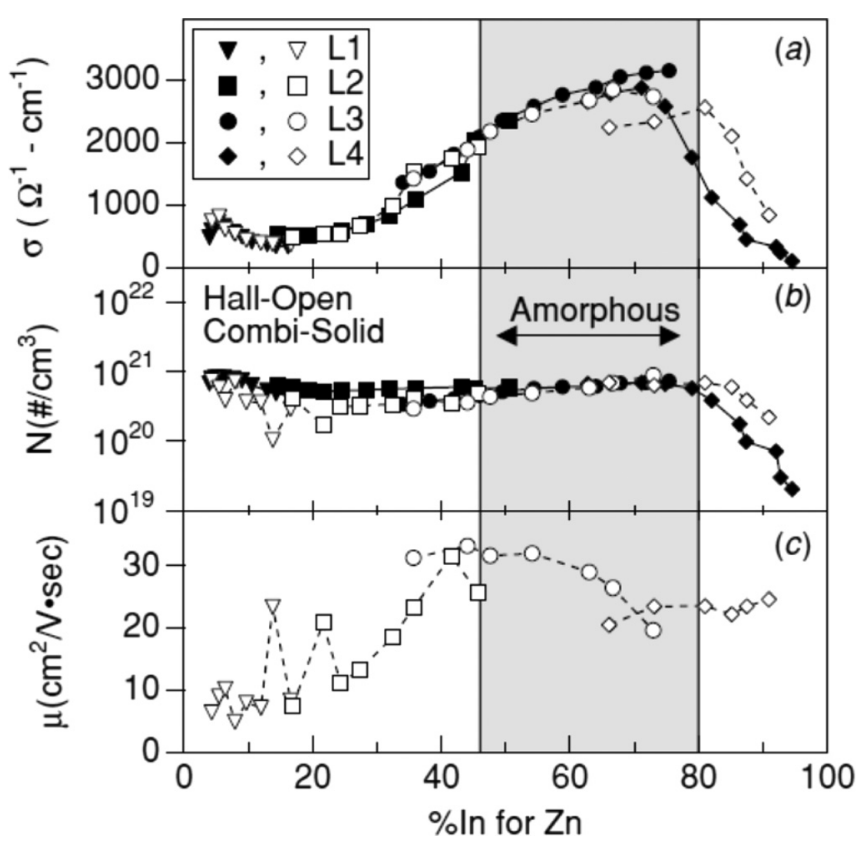

FIG. 50. Comparison of conductivity (a), carrier concentration (b), and mobility as measured by combinatorial tools (closed symbols) and discrete Hall measurements (open symbols). A broad maximum in conductivity and mobility is observed in the region determined to be amorphous by XRD. Reproduced with permission from M. P. Taylor et al., Meas. Sci. Technol. 16, 90 (2005). Copyright 2005 Institute of Physics.

There has also been interest in doping $\mathrm{In}_{2} \mathrm{O}_{3}$ with TM such as $\mathrm{Ti}$ and Mo to further increase conductivity. In In-Mo-O libraries, it was found that for films deposited at $350{ }^{\circ} \mathrm{C}$, the maximum conductivity was only $1000(\Omega-\mathrm{cm})^{-1}$ for low concentrations $\left(\sim 6\right.$ at $\%$ ) of $\mathrm{Mo} ;{ }^{198}$ this maximum is attributable to the opposing compositional trends of carrier concentration and electron mobility. Samples annealed at $550{ }^{\circ} \mathrm{C}$ showed a six-fold increase in conductivity to $6000(\Omega$ $\mathrm{cm})^{-1}$, although such thermal treatment makes the material incompatible with organic photovoltaics and raises some questions about stability when deposited on $\mathrm{Si}$ - or CdTebased photovoltaics. In-Ti-O films were also reported in the same study; in this case, samples annealed at $500{ }^{\circ} \mathrm{C}$ exhibited a maximum conductivity of $6000(\Omega \mathrm{cm})^{-1}$ for Ti concentrations between 3 and 4 at. $\%{ }^{200}$ Between 1.5 and 3 at. $\%$ Ti doping, a linear relationship between $\mathrm{Ti}$ concentration and carrier density was observed, expressed as 1.06 electrons/Ti atom, Fig. 51. This is about a factor of five larger than the trend seen for Mo, and suggests that $\mathrm{Ti}$ is a promising dopant for $\mathrm{In}_{2} \mathrm{O}_{3}$.

Cosputtering through a mask was used to create a library of discrete zinc indium tin oxide (ZITO) CCS films on polymeric substrates. ${ }^{202}$ The samples were deposited from a pure $\mathrm{ZnO}$ target and an $\mathrm{In}_{2} \mathrm{O}_{3}$ target doped with $10 \mathrm{wt}$. \% Sn. The Van der Pauw method was used to measure the resistivity, carrier concentration, and mobility of each discrete film. XRD revealed samples that were amorphous across the entire composition region investigated. A maximum conductivity of $2.4 \times 10^{3}(\Omega-\mathrm{cm})^{-1}$ was observed at a $\mathrm{Zn}$ content of 8.9 at. \%; this value is comparable to values for crystalline $\mathrm{In}_{2} \mathrm{O}_{3}$. A negligible compositional dependence on carrier concentration was observed, decreasing only slightly for

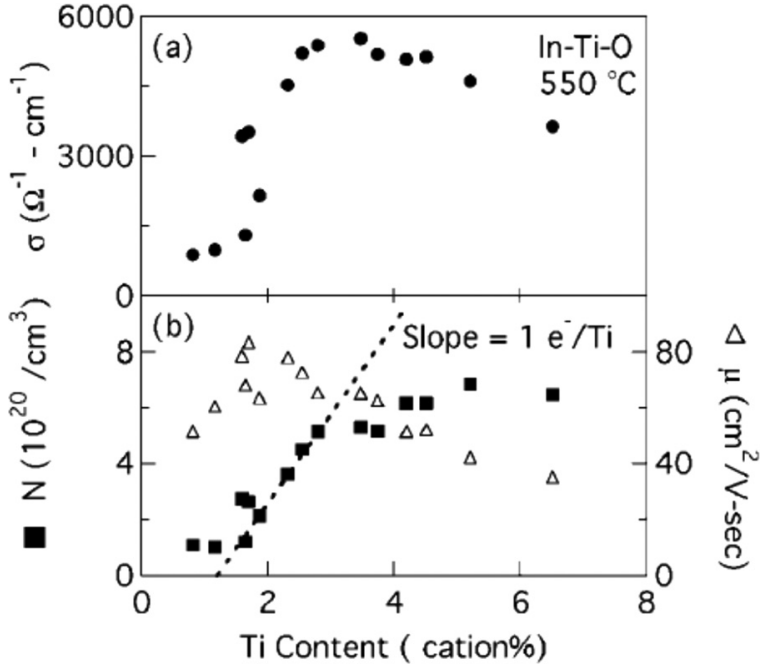

FIG. 51. Conductivity, carrier concentration, and mobility measured as a function of Ti content in $\mathrm{In}_{2} \mathrm{O}_{3}$ films. The carrier concentration varies linearly over a range of 2 to 4 at. $\% \mathrm{Ti}$, with the conductivity peaking in this region. Reprinted with permission from M. van Hest et al., Appl. Phys. Lett. 87, 032111 (2005). Copyright 2005 American Institute of Physics.

increased $\mathrm{Zn}$ content. In contrast, the carrier mobility was observed to show a strong dependence on the overall $\mathrm{Zn}$ content. For all compositions investigated, the optical transmission of ZIO in the wavelength range $300 \mathrm{~nm}$ to $2400 \mathrm{~nm}$ was observed to be larger than the for conventionally grown ITO films. Finally, PLD was used to synthesize libraries in the $\mathrm{In}_{2-2 \mathrm{x}} \mathrm{Me}_{2 \mathrm{x}} \mathrm{O}_{3}$ system, where $\mathrm{Me}=\mathrm{Ti}, \mathrm{Zr}$, and $\mathrm{Sn}^{203}$ It was found that $\mathrm{Ti}$ and $\mathrm{Zr}$ additions to $\mathrm{In}_{2} \mathrm{O}_{3}$ yielded superior optical properties compared to those incorporating $\mathrm{Sn}$, without compromising resistivity.

\section{2. $\mathrm{SnO}_{2}$-based TCOs}

$\mathrm{SnO}_{2}$ is the most commonly utilized TCO film material, due to its large band gap $(4.1 \mathrm{eV})$ and the high natural abundance of $\mathrm{Sn} . \mathrm{SnO}_{2}$ is generally doped with either fluorine $(\mathrm{F})$ or antimony ( $\mathrm{Sb})$ to increase its conductivity to about $1.7 \times 10^{3}(\Omega-\mathrm{cm}),{ }^{-1}$ although this is still more than a factor of two smaller than for ITO. Despite its greater resistance, the low cost of Sn has led to a large effort in identifying optimal doping and processing conditions for $\mathrm{SnO}_{2}$. Optimization of F-doped $\mathrm{SnO}_{2}$ has been reported using atmospheric pressure chemical vapor deposition (APCVD), with $\mathrm{SnCl}_{4}$ and aqueous hydrogen fluoride as precursors. ${ }^{204}$ The goal of this study was to optimize the growth of the $\mathrm{SnO}_{2}: \mathrm{F}$ using high throughput techniques, followed by implementation of the optimized films in photovoltaic cells. The combinatorial APCVD experiment was realized through the use of a movable susceptor, which heated local areas of a large wafer; this enabled serial growth of films at different temperatures and precursor mixtures. Two different types of samples were grown in this study. In the first set, the TCOs were grown on glass substrates for measurement of resistivity, transparency, surface roughness, and optical absorption of the $\mathrm{SnO}_{2}$ processed under different conditions. It was found that under appropriate conditions, the sheet resistance could be lowered to acceptable levels, with optical 
absorption losses of $1 \%$ at $500 \mathrm{~nm}$ wavelength. ${ }^{205}$ Additionally, it was found that optimal film morphologies could be obtained through the controlled addition of methanol and t-butanol. ${ }^{206}$ In the second set of samples, the deposition of the TCO layer was followed by the growth of a standard a-Si:H single junction p-i-n solar cell, ${ }^{207}$ Fig. 52. Then, the full quantum efficiency of a library of solar cells incorporating $\mathrm{SnO}_{2}$ grown at different conditions was measured using a calibrated light source (i.e., the light source tests the solar cell response vs. the solar spectrum when the direct optical path length through the Earth's atmosphere is 1.5 times as long as the path length at the zenith). Energy conversion efficiencies of up to $9.3 \%$ with high short-circuit currents $\left(14.9 \mathrm{~mA} / \mathrm{cm}^{2}\right)$ and external quantum efficiency (i.e., the proportion of photons that produce collected charge carriers) values of $86 \%$ at $540 \mathrm{~nm}$ wavelength were achieved.

\section{ZnO-based TCOs}

Other commonly used TCOs are based on the $\mathrm{ZnO}$ system. With appropriate doping $\mathrm{ZnO}$ has been reported to have conductivities as high as $5 \times 10^{3}(\Omega-\mathrm{cm})^{-1}$, comparable to ITO, and $\mathrm{Zn}$, like $\mathrm{Sn}$, is much less expensive than In. An additional benefit is that $\mathrm{ZnO}$ exhibits high thermal and chemical stability. To date, there have been combinatorial studies for various dopants (gallium $(\mathrm{Ga})$, aluminum $(\mathrm{Al})$, nitrogen $(\mathrm{N})$, and cobalt (Co)) in $\mathrm{ZnO}$, and the alloying of $\mathrm{ZnO}$ with $\mathrm{SnO}$. Experiments were carried out on $\mathrm{ZnO}$ doped with $\mathrm{N}$ and $\mathrm{Al}$ to demonstrate in situ materials processing monitoring of TCOs during annealing. ${ }^{193}$ It was found that high spatial resolution (10 $\mu \mathrm{m}$ spot size) fiber optic reflectance measurements of individual spots on a library during annealing could be obtained in less than $15 \mathrm{~ms}$, Fig. 53. This measurement system was then applied ${ }^{208}$ to investigate the properties of $\mathrm{ZnO}$ alloyed with $\mathrm{SnO}_{2}$, in compositions along the $\mathrm{ZnO}-\mathrm{SnO}_{2}$ tie line. Two local maxima in conductivity were reported, for $\mathrm{Zn} / \mathrm{Sn}$ ratios of $2: 1$ and $1: 1$, corresponding to the formation of the amorphous compositions $\mathrm{Zn}_{2} \mathrm{SnO}_{4}$ and $\mathrm{ZnSnO}_{3}$; both phases showed good transmission $(\sim 80 \%)$. Other researchers ${ }^{197}$ used sol-gel methods to dope $\mathrm{ZnO}$ with $\mathrm{Al}$. After annealing at $1073 \mathrm{~K}, \mathrm{XRD}$ peaks attributed to $\mathrm{ZnAl}_{2} \mathrm{O}_{4}$ were identified for $\mathrm{Al}$ contents as low as 2 at. \%, and UV/VIS measurements demonstrated transmissions as high as $80 \%$ at $550 \mathrm{~nm}$. Using

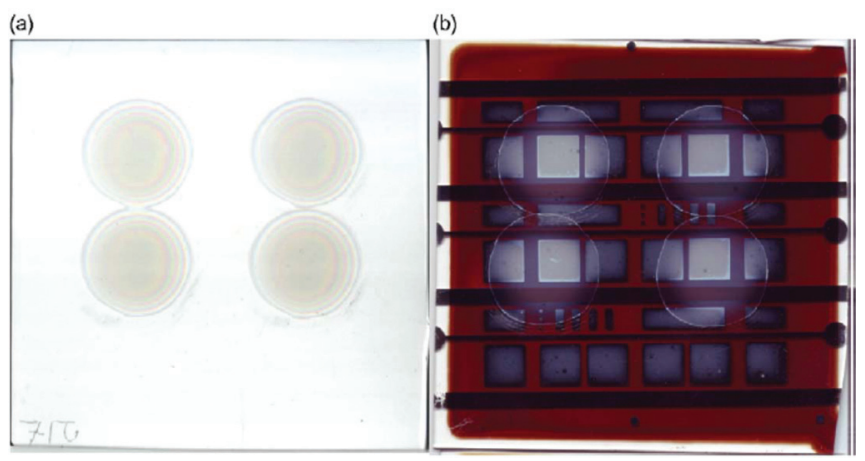

FIG. 52. Images of $\mathrm{F}$ doped $\mathrm{SnO}_{2}$ samples deposited by APCVD on (a) a glass substrate and (b) as a series of solar cells. Reproduced with permission from U. Dagkaldiran et al., Mater. Sci. Eng., B. 159-160, 6 (2009). Copyright 2009 Elsevier S.A.

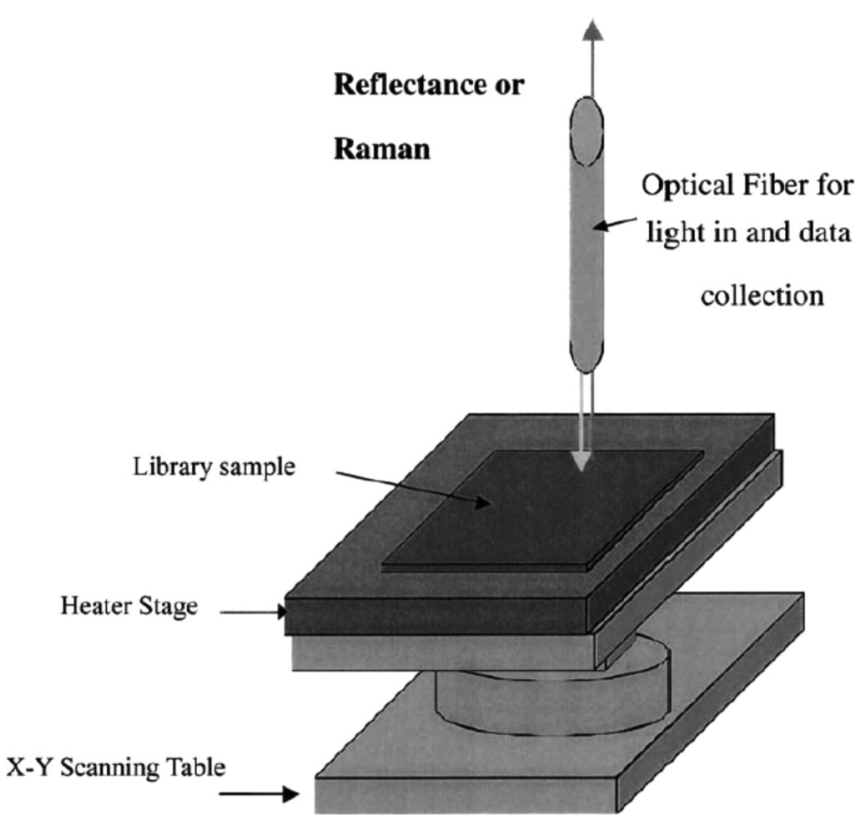

FIG. 53. Schematic of high spatial resolution in situ reflectance measurement system. Reproduced with permission from Q. Wang et al., Appl. Surf. Sci. 189, 271 (2002). Copyright 2002 Elsevier BV.

robot assisted sol-gel methods, a series of mixtures of $\mathrm{Zn}-\mathrm{Sn}$ $\mathrm{O}$ along the same tie line were prepared in further work. ${ }^{209}$ Their results showed that in contrast to PVD and CVD methods, sol-gel samples showed unacceptably low conductivities.

Substitutional doping of $\mathrm{ZnO}$ with $\mathrm{Ga}$ via combinatorial sputtering was also studied, ${ }^{210}$ with maximum Ga contents up to 8 at. $\%$. The position of the conductivity peak was found to shift to lower Ga concentrations with increasing annealing temperature, with a maximum value of $1.4 \times 10^{-3}$ $(\Omega-\mathrm{cm})^{-1}$ for samples containing 7.5 at. $\%$ Ga annealed at $250{ }^{\circ} \mathrm{C}$, Fig. 54. Further, transmissivities were greater than $80 \%$ for the entire library. Jung et al. ${ }^{211}$ reported doping of highly oriented (002) $\mathrm{ZnO}$ films doped with $\mathrm{Ga}_{2} \mathrm{O}_{3}$. The libraries were grown on glass substrates at RT, and focused on compositions with less than 13.6 wt. $\% \mathrm{Ga}_{2} \mathrm{O}_{3}$. They

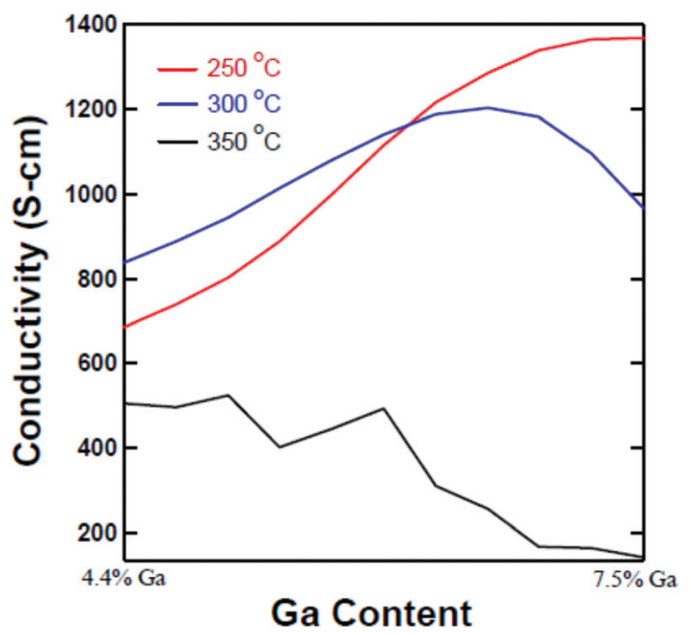

FIG. 54. Compositional variation of conductivity in $\mathrm{ZnO}$ samples doped with $\mathrm{Ga}$ at different deposition temperatures. Reproduced with permission from C. W. Gorrie et al., 33rd IEEE Photovoltaic Specialists Conference (2008), Vol. 1-4, p. 635. Copyright 2008 IEEE. 
report an order of magnitude decrease in the conductivity, from $\sim 10^{3}(\Omega \mathrm{cm})^{-1}$ for the lowest $\mathrm{Ga}_{2} \mathrm{O}_{3}$ concentrations, to $\sim 10^{2}(\Omega \mathrm{cm})^{-1}$ in samples with $7.0 \mathrm{wt}$. \%, Fig. 55 . The best sample contained 0.8 wt. $\% \mathrm{Ga}_{2} \mathrm{O}_{3}$ and had a conductivity $6.8 \times 10^{2}(\Omega \mathrm{cm})^{-1}$ with an optical transmittance at $550 \mathrm{~nm}$ of $90 \%$. A direct comparison of the maximum conductivities $^{210,211}$ of materials with similar compositions shows a factor of two differences in the values reported, while the values and trends for optical transmission compare well at RT.

In an effort to explore p-type doping of $\mathrm{ZnO}$, combinatorial studies of $\mathrm{Co}$ doping of $\mathrm{ZnO}$ have been conducted. Co has a similar ionic radius to $\mathrm{Zn}$ and thus has a large solubility. Further, there is the potential that the $\mathrm{d}^{6}$ electron configuration of $\mathrm{Co}^{3+}$ in an octahedral crystal environment could aid in providing a conduction path for hole carriers at the top of the valence band. Suh et al. produced a series of combinatorial libraries at different oxygen partial pressures and correlated structural information gathered by XRD with composition through the use of principle components analysis. ${ }^{212}$ From this, it was determined that the presence of oxygen vacancies in the samples was related not only to the oxygen incorporation rate during deposition but also to the overall Co content in the film. The highest conductivity samples were those of the structure type $\mathrm{ZnCo}_{2} \mathrm{O}_{4}$.

\section{Other TCO systems}

Combinatorial libraries of the IR transparent, TCO $\mathrm{NiO}-\mathrm{Mn}_{2} \mathrm{O}_{3}-\mathrm{Co}_{3} \mathrm{O}_{4}$ ternary system were screened for their conducting properties as a function of temperature. ${ }^{213}$ Conductivity measurements were performed with $80 \mu \mathrm{m}$ resolution by using a carefully selected sharp graphite rod, which was kept in constant contact with the film during scanning. By controlling the hardness of the graphite, electrical contact could be maintained without scratching the film surface or damaging the probe tip. They found that the conductivities of a series of ternary alloys along the line $\mathrm{Mn}_{0.45} \mathrm{Ni}_{0.63}$ $\mathrm{Co}_{1.92} \mathrm{O}_{4}-\mathrm{Mn}_{0.60} \mathrm{Ni}_{0.72} \mathrm{Co}_{1.68} \mathrm{O}_{4}-\mathrm{Mn}_{0.69} \mathrm{Ni}_{0.81} \mathrm{Co}_{1.5} \mathrm{O}_{4}$, while comparable to $\mathrm{NiCo}_{2} \mathrm{O}_{4}$, exhibited much lower temperature sensitivity (about $1.5 \% / \mathrm{K}$ ).

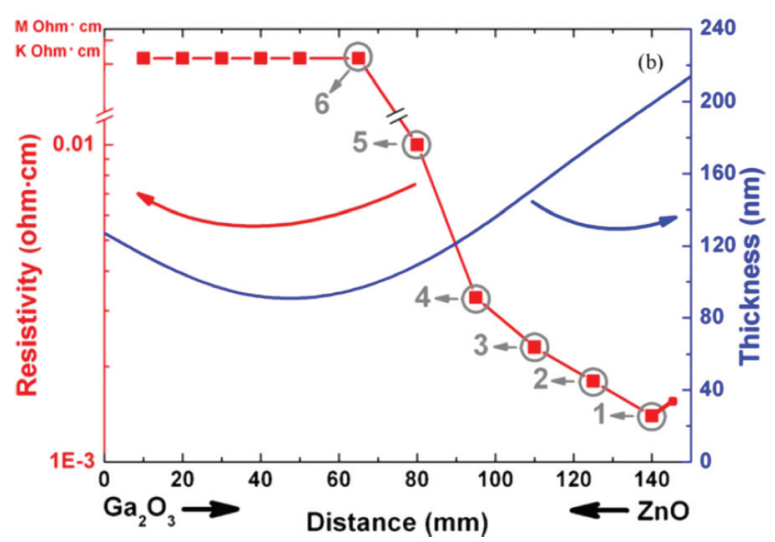

FIG. 55. Resistivity and film thickness as a function of position on a $\mathrm{ZnO}-\mathrm{Ga}_{2} \mathrm{O}_{3} \mathrm{CCS}$ library. Reproduced with permission from K. Jung et al., Appl. Surf. Sci. 256, 6219 (2010). Copyright 2010 Elsevier BV.
Further research used PLD to deposit CCS libraries of Nb-Sn-Ti-O. ${ }^{214}$ Their results showed that films with Sn levels less than 20 at\% maintained low resistivity and high transparency, while reducing the refractivity of the coating (a reduction in the refractivity reduces the effective path length of light through a material, thus reducing the probability of absorption). Using metal-organic chemical vapor deposition (MOCVD), linearly varying CCS libraries of Co-Sn-O were deposited. $^{215}$ In this work, transmission greater than $80 \%$ from $500 \mathrm{~nm}$ to $1000 \mathrm{~nm}$ wavelengths across the entire library was reported. Using standard Hall measurement techniques, high carrier concentrations $\left(10^{20} \mathrm{~cm}^{-3}\right)$ and mobilities $\left(60 \mathrm{~cm}^{2} \mathrm{~V}^{-1} \mathrm{~s}^{-1}\right)$ were found for all compositions.

\section{Phase change materials}

Phase-change materials (PCMs), most often semiconducting or semi-metallic alloys (also known as chalcogenide compounds) containing $\mathrm{S}, \mathrm{Se}$, or $\mathrm{Te}$, have both optical and electrical storage applications. ${ }^{216}$ Upon heating, PCMs undergo a reversible phase transformation from a supercooled, metastable amorphous phase to a crystalline phase. The amorphous phase has significantly lower reflectivity than the crystalline phase, making them the material of choice for rewriteable optical storage media (e.g., compact discs). ${ }^{217,218}$ PCMs also have a low electrical resistance in the crystalline phase, which may increase by many orders of magnitude in the amorphous phase, making them very useful for storage applications as non-volatile flash memories in integrated circuits. ${ }^{219}$ In fact, the original intended application for PCMs was for integrated circuit memory storage. $^{220,221}$

To achieve high write and erase rates for memory storage, the kinetics of the phase transformation between the amorphous and crystalline phases (in both directions) must be very fast. Non-combinatorial crystallization kinetics studies led to the selection of Ge-Sb-Te ternary alloys for optical storage applications. ${ }^{222,223}$ Alloys for nonvolatile memory applications also require fast kinetics, but other factors such as low power consumption and cycling endurance are also important. $\mathrm{Ge}_{2} \mathrm{Sb}_{2} \mathrm{Te}_{5}$ is the alloy of choice for this application at this time. However, since a large range of composition space is being explored for this application, it is interesting to note that there is a dearth of combinatorial experimentation in this area. It is likely that the data required for this application are not readily obtained by combinatorial methods, or that much of this work is proprietary and has not been published. One group performed combinatorial investigations $^{224}$ on sputter deposited Ge-Sb-Te films. The CCS film library had concentration gradients centered about the $\mathrm{Ge}_{2} \mathrm{Sb}_{2} \mathrm{Te}_{5}$ phase. Crystallization times that varied between 220 and $500 \mathrm{~ns}$, and write times that varied between 20 and $120 \mathrm{~ns}$, both as a function of composition, were measured. Figure 56 illustrates a map of amorphization (writing) time as a function of composition. Further work by this group ${ }^{225}$ expanded the materials optimization to CCS libraries based around three other compounds: $\mathrm{GeSb}_{4} \mathrm{Te}_{7}, \mathrm{GeSb}_{2} \mathrm{Te}_{4}$, and $\mathrm{Ge}_{4} \mathrm{SbTe}_{5}$. It was determined that ternary compositions at or 


\section{amorphization}

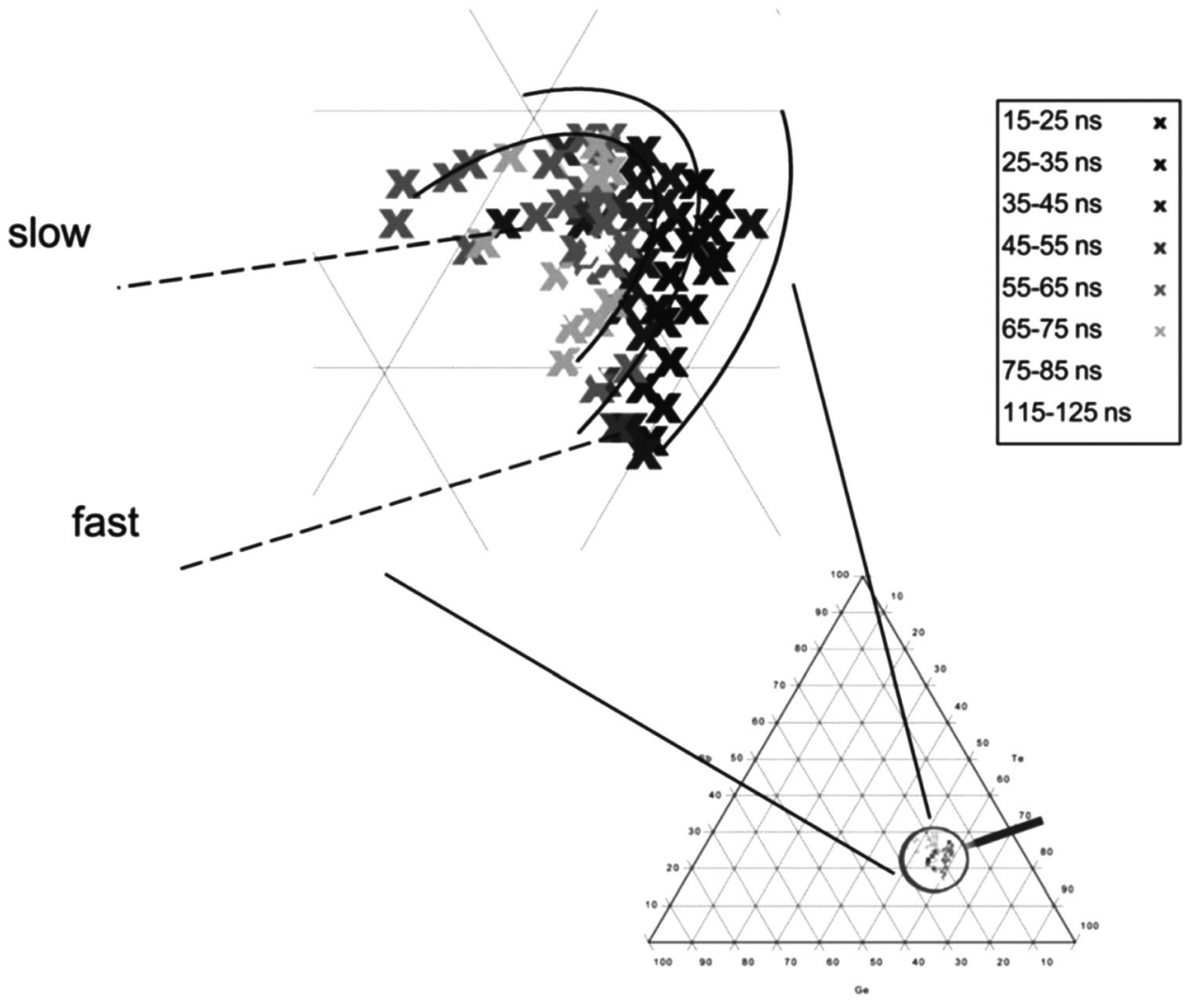

FIG. 56. Map of amorphization (writing) time on a Ge-Sb-Te CCS film library. Reproduced with permission from S. Kyrsta et al., Thin Solid Films 398, 379 (2001). Copyright 2001 Elsevier S.A.

near the GeTe- $\mathrm{Sb}_{2} \mathrm{Te}_{3}$ pseudobinary tie line exhibit fast (70-150 ns) crystallization behavior.

\section{ENERGY-RELATED MATERIALS}

\section{A. Thermoelectrics}

Thermoelectric materials, which enable the direct solid state conversion of thermal to electrical energy, have important applications for utilization of waste heat energy (particularly vehicular engine heat) via the Seebeck effect, as well as solid state refrigeration via the Peltier effect. ${ }^{226,227}$ However, at present, the best thermoelectric conversion materials and devices are not efficient enough for widespread commercial use. The availability of higher conversion efficiency thermoelectric materials will play a significant role in their implementation. High conversion efficiency thermoelectric devices require materials that possess large figures of merit, $\mathrm{ZT}$, which is equal to $\mathrm{S}^{2} \sigma \mathrm{T} / \kappa$, where $\mathrm{S}$ the Seebeck coefficient, $\sigma$ the electrical conductivity, $\kappa$ the thermal conductivity, and $\mathrm{T}$ the absolute temperature. Combinatorial approaches have been used to optimize and discover thermoelectric materials through measurement of $\mathrm{S}, \sigma$, and therefore $S^{2} \sigma$ (another thermoelectric figure of merit, known as the power factor). To obtain ZT, the thermal conductivity of the film must also be measured. This is a notoriously difficult measurement to perform, especially on a combinatorial thin film library, because the mass of the substrate is far greater than that of the film; however, high throughput measurements of thermal conductivity have also been performed, as will be discussed below.
Using PLD, a CCS library film of $\left(\mathrm{Ca}_{1-\mathrm{x}} \mathrm{Ba}_{\mathrm{x}}\right)_{3} \mathrm{Co}_{4} \mathrm{O}_{9}$ was prepared, where $\mathrm{x}$ varied between 0 and $0.2 .^{228}$ The Seebeck coefficient and resistivity of the striped and patterned films in the library were then measured using a multichannel four point probe array, Fig. 57, where one side of the film stripe was heated to create the required $\Delta \mathrm{T}$ for measurement, and the other side was maintained at RT. Barium substitution was found to significantly decrease

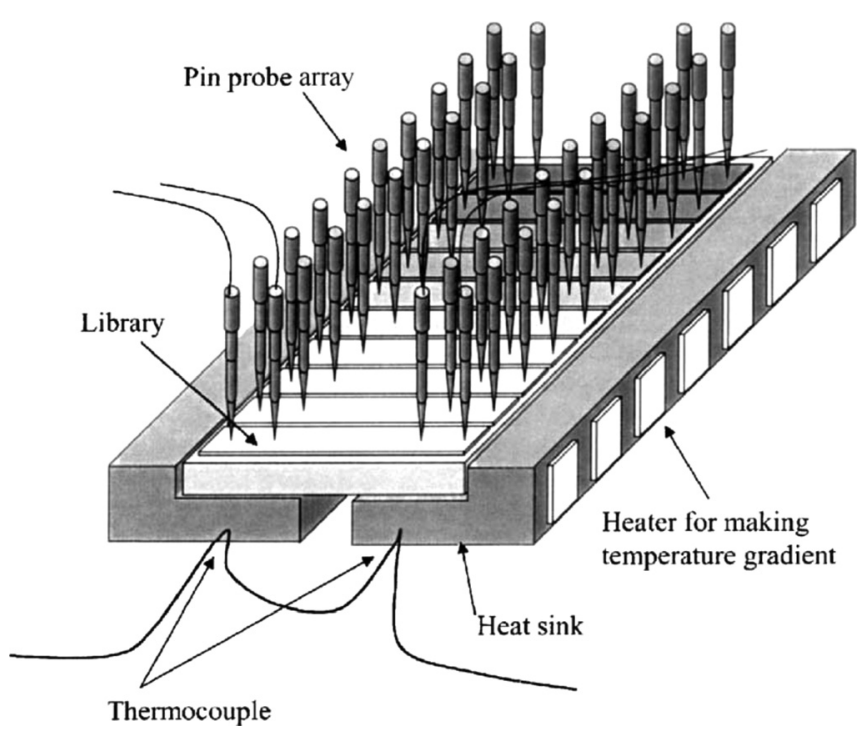

FIG. 57. Schematic diagram of a multichannel power factor measurement system for thermoelectric materials. Reproduced with permission from $\mathrm{H}$. Minami et al., Appl. Surf. Sci. 197, 442 (2002). Copyright 2002 Elsevier BV. 


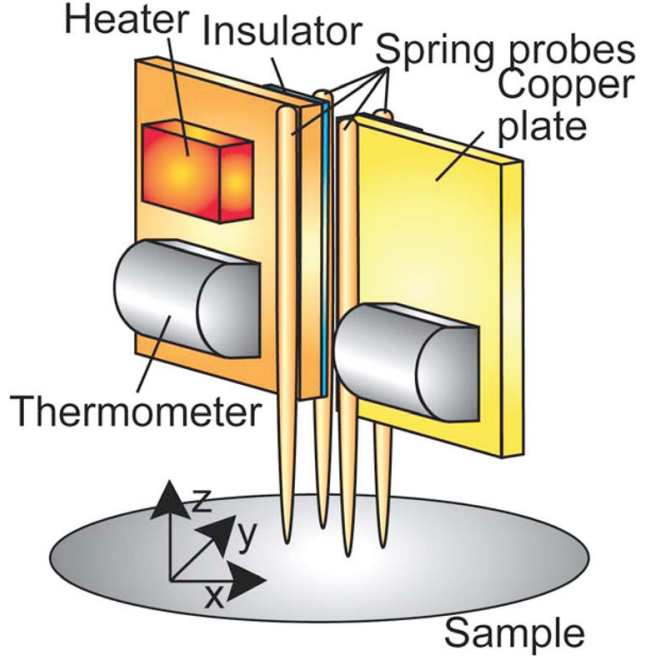

FIG. 58. Schematic diagram of measurement probe to measure electrical conductivity and Seebeck coefficient. Reprinted with permission from M. Otani et al., Appl. Phys. Lett. 91, 132102 (2007). Copyright 2007 American Institute of Physics.

the conductivity, but had no effect on the Seebeck coefficient. The $\mathrm{Sr}$ and $\mathrm{La}$ substituted $\mathrm{Ca}_{3} \mathrm{Co}_{4} \mathrm{O}_{9}$ system, $\left(\mathrm{Ca}_{1-\mathrm{x}-\mathrm{y}} \mathrm{Sr}_{\mathrm{x}} \mathrm{La}_{\mathrm{y}}\right)_{3} \mathrm{Co}_{4} \mathrm{O}_{9}$, where $\mathrm{x}$ varied continuously between 0 and approximately 0.35 , has also been studied combinatorially. ${ }^{229,230}$ PLD was used to deposit the ternary film library, and a four point screening probe, with one heated leg, Fig. 58, was used to measure both the Seebeck coefficient and the electrical resistivity. The probe spatial resolution was about $1 \mathrm{~mm}$; over one thousand points in the library were measured in about $6 \mathrm{~h}$. Seebeck coefficient calibration films measured with this probe were found to be in good agreement with those measured using a commercial thermoelectric measurement apparatus. The rapid construction of a thermoelectric property diagram is illustrated in Fig. 59 for the $\mathrm{Ca}_{3-\mathrm{x}-\mathrm{y}} \mathrm{Sr}_{\mathrm{x}} \mathrm{La}_{\mathrm{y}} \mathrm{Co}_{4} \mathrm{O}_{9}$ system. One can see that the power factor "sweet spot" is easily identified at about $\mathrm{x}=0.25$ and $\mathrm{y}=0.10$. Such high throughput thermoelectric materials analysis will likely facilitate commercialization of this class of materials.

Another high throughput technique for thermoelectric oxides has been reported ${ }^{231,232}$ in which thick film discrete libraries were synthesized by depositing aqueous solutions of $\mathrm{M}_{1}-\mathrm{M}_{2}-\mathrm{Co}-\mathrm{O}(\mathrm{M}=$ metal $)$ on an alumina plate, followed by

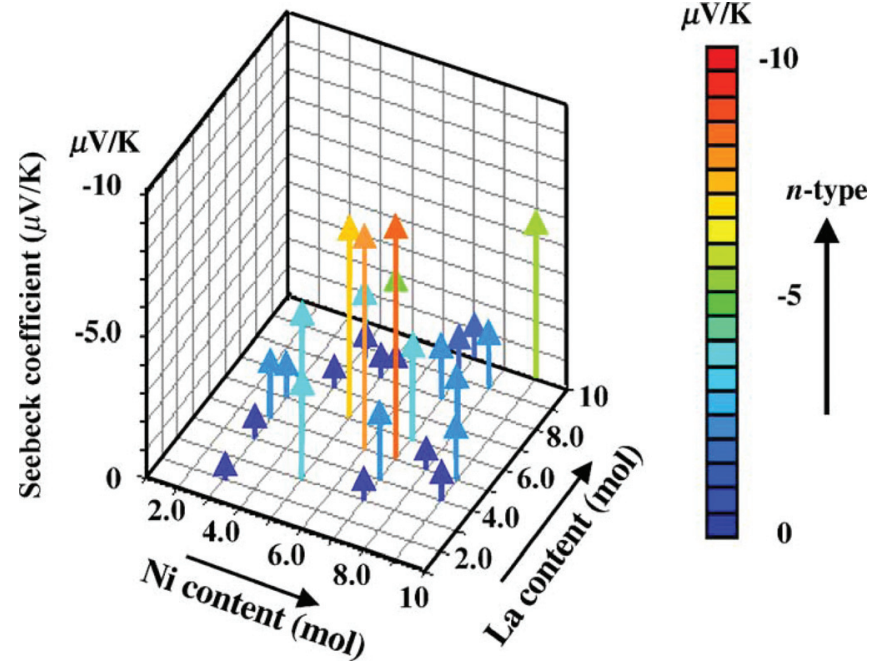

FIG. 60. Graph of Seebeck coefficients from the La-Ni-O library heated at $1073 \mathrm{~K}$ for $10 \mathrm{~h}$ in air. Reproduced with permission from R. Funahashi et al., Appl. Surf. Sci. 223, 44 (2004). Copyright 2004 Elsevier BV.

annealing. The macroscopic films, approximately one centimeter in length, were then probed with a handheld two terminal Seebeck probe. This technique enabled the screening of up to about 1000 samples per day. An effective example of its use for the La-Ni-O system is shown in Fig. 60.

Another class of thermoelectric materials that was studied is the $\mathrm{Mg}_{\mathrm{x}} \mathrm{Si}_{\mathrm{y}} \mathrm{Ge}_{1-\mathrm{y}}$ ternary system, where $\mathrm{x}$ was varied between 2.3 and 4.5, and y between 0.45 and 1, on a discrete PLD film library sample. ${ }^{233}$ The library was deposited onto a prefabricated alumina substrate into which the local heater and contact leads had been integrated. Temperature gradients across the sample, as well as the base temperature of the samples (which varied between RT and $673 \mathrm{~K}$ ), were measured with an IR camera. The measurements were first calibrated using a constantan film, whose Seebeck coefficient and resistivity were found to be very close to the bulk values. Seebeck coefficient and resistivity results for this ternary library film are depicted in Fig. 61.

More traditional thermoelectric metal alloys have also been studied using high throughput methods. ${ }^{234} \mathrm{Ni}_{1-\mathrm{x}} \mathrm{Cu}_{\mathrm{x}}$ library samples, where $\mathrm{x}$ spanned the entire range from 0 to 1 , were prepared in two different ways. The first set was prepared by powder metallurgy techniques involving ball milling of eleven different compositions, followed by spark

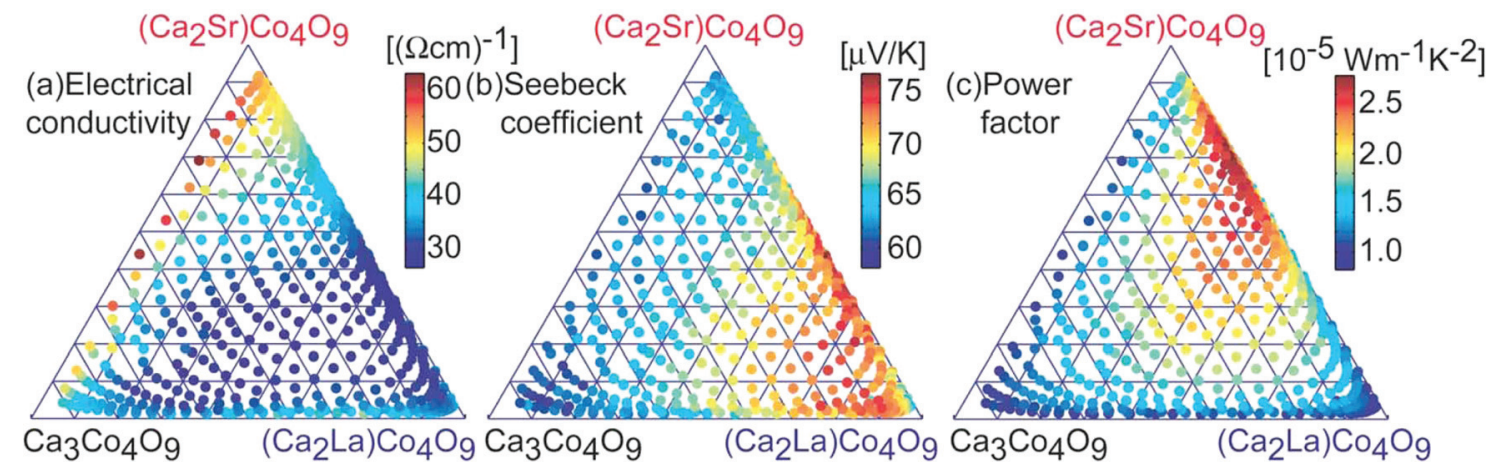

FIG. 59. (a) Electrical conductivity, (b) Seebeck coefficient, and (c) power factor of the library film $\left(\mathrm{Ca}_{1-x-y} \mathrm{Sr}_{x} \mathrm{La}_{y}\right)_{3} \mathrm{Co}_{4} \mathrm{O}_{9}$, where $\mathrm{x}$ and $\mathrm{y}$ vary between 0 and approximately 0.35. Reprinted with permission from M. Otani et al., Appl. Phys. Lett. 91, 132102 (2007). Copyright 2007 American Institute of Physics. 


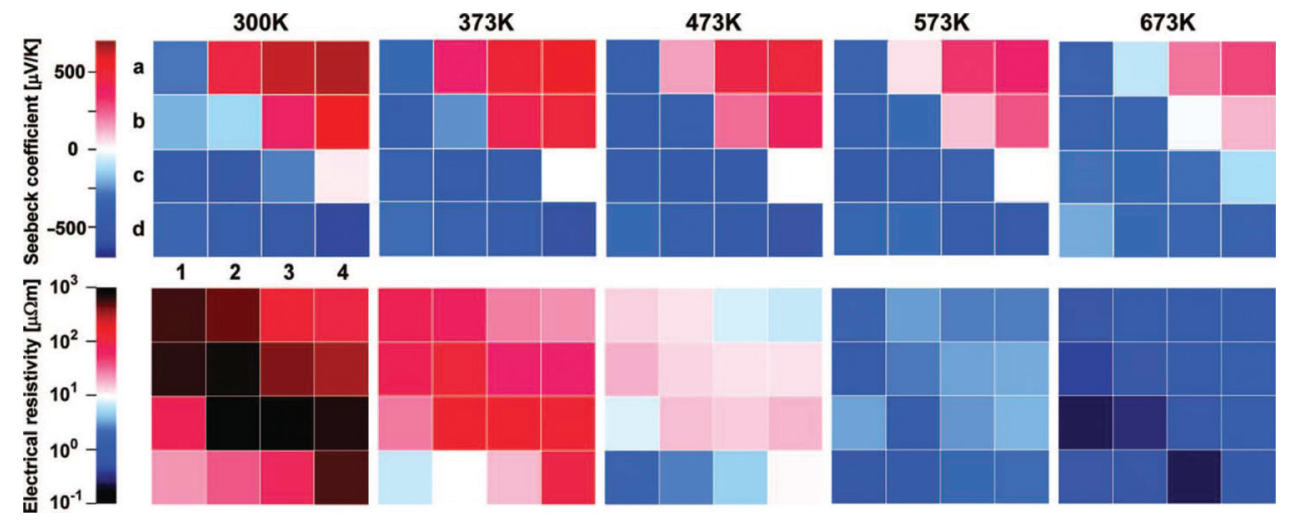

FIG. 61. Compositional dependence of the Seebeck coefficient and electrical resistivity at different temperatures for the Mg-Si-Ge library. (a-d) and (1-4) refer to various compositional points on the library film. Reproduced with permission from M. Watanabe et al., J. Comb. Chem. 10, 175 (2008). Copyright 2008 American Chemical Society.

sintering of powder layers of different compositions to form a bulk sample with a compositional gradient. This bulk library sample was then sliced into thin wafers to expose the compositional gradient. In the second method, discrete compositions were formed in microwells in a graphite sample holder by melting and slicing the pellets. The thermoelectric properties of the $\mathrm{Ni}_{1-\mathrm{x}} \mathrm{Cu}_{\mathrm{x}}$ alloys were measured from $323 \mathrm{~K}$ to $950 \mathrm{~K}$, and the results are shown in Fig. 62. In particular, $\mathrm{Ni}_{0.7} \mathrm{Cu}_{0.3}$ is found to possess a large power factor, $0.012 \mathrm{Wm}^{-1} \mathrm{~K}^{-2}$ at around $950 \mathrm{~K}$ (calculable from Figs. 62(a) and 62(b).

In addition to the power factor figure of merit, $S^{2} \sigma$, whose components are reasonably easy to measure (even combinatorially), the more complete thermoelectric power factor, ZT (equal to $\mathrm{S}^{2} \sigma \mathrm{T} / \kappa$ ) is often desired. Thus, the thermal conductivity, $\kappa$, must be determined. Using Ni-based bulk diffusion multiple sample libraries, Fig. 63, thermal conductivity of $\mathrm{Ni}$ alloys were determined combinatorially by time domain thermoreflectance (TDTR) measurements, with a spatial resolution of about four micrometers, and a sampling rate of 3 points/s. ${ }^{235}$ The authors concluded that their measured thermal and electrical conductivities for the $\mathrm{Ni}$ alloys could not be well described by theory, as their measured and calculated values deviated by as much as $11 \%$ to $29 \%$, depending upon the alloy. Thus, their combinatorial experiments were absolutely necessary as a consistency check on theoretical values. It is also important to note that their data, Fig. 64, show excellent consistency with noncombinatorially determined values for certain alloys.

High throughput TDTR measurements have also been extended to oxide thin films. ${ }^{230}$ In this case, thermal effusivity, $b$, equal to $(\kappa c \rho)^{1 / 2}$ (where $\kappa$ is the thermal conductivity, $\mathrm{c}$ is the specific heat, and $\rho$ is the density), was measured on a metal coated oxide film, with a ten micrometer spot size. The authors were able to obtain a thermal effusivity calibration curve using various bulk samples, i.e., $\mathrm{SiO}_{2}, \mathrm{SrTiO}_{3}, \mathrm{LaAlO}_{3}, \mathrm{Al}_{2} \mathrm{O}_{3}$, and $\mathrm{Si}$. Using this calibration, the thermal conductivity of an yttria $\left(\mathrm{Y}_{2} \mathrm{O}_{3}\right)$ containing ternary oxide film was then determined. The measured value, $11.96 \mathrm{~J}(\mathrm{Kms})^{-1}$, was within about $10 \%$ of the reported value of $12.87 \mathrm{~J}(\mathrm{Kms})^{-1}$. This tool will clearly be a very useful thermoelectric (as well as other materials) screening tool.

\section{B. Lithium battery materials}

Battery powered high technology products are ubiquitous. Since the advent of rechargeable nickel metal hydride $(\mathrm{NiMH})$ batteries enabled environmentally friendly portable electronics, the market for rechargeable batteries has dramatically expanded. ${ }^{236,237} \mathrm{Li}$ ion batteries have emerged as the preferred power source for portable electronics, laptops, cell phones, and high performance electric vehicles due to their superior gravimetric and volumetric energy densities, reduced self-discharge, improved cycling capabilities, and thermal stability. State of the art Li ion batteries exhibit remarkable characteristics, with the following properties (maxima) reported: energy storage capacities of $2400 \mathrm{~mA}-\mathrm{h}$; specific energies of $250 \mathrm{~W}-\mathrm{h} / \mathrm{kg}$; energy densities of $620 \mathrm{~W}-\mathrm{h} / \mathrm{l}$; and specific powers of $340 \mathrm{~W} / \mathrm{kg}$. Further, they can be cycled between four and twelve hundred times. Typical cells are composed of a graphite anode that intercalates $\mathrm{Li}$ ions given off by a $\mathrm{LiCoO}_{2}$ cathode during discharge.
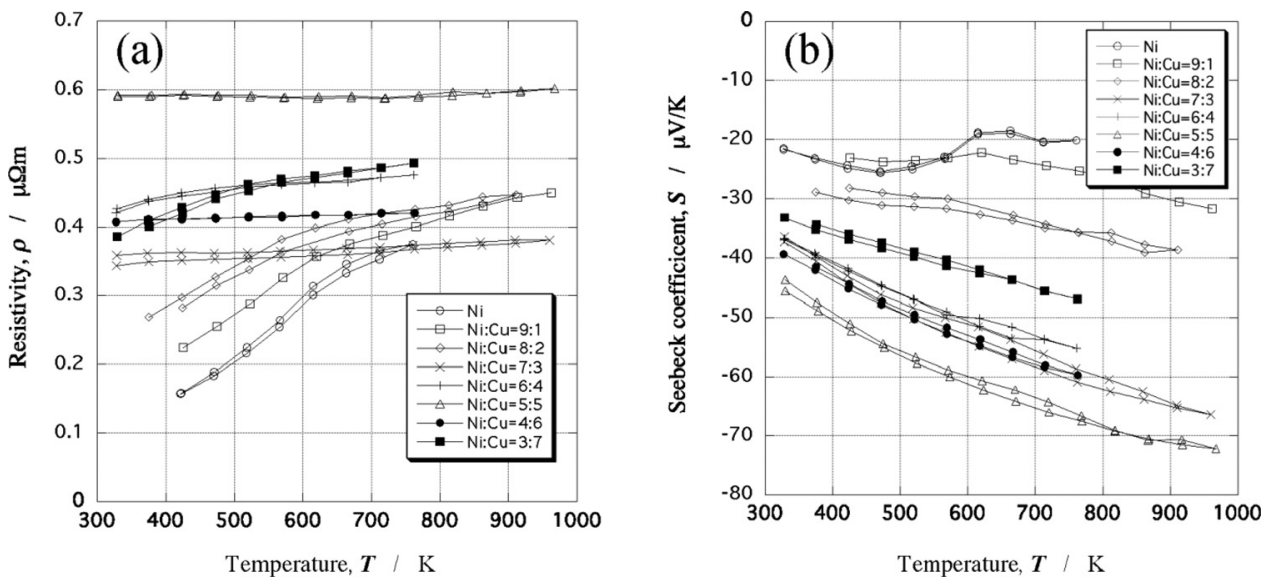

FIG. 62. The temperature dependence of resistivity (a) and Seebeck coefficient (b) of Ni-Cu alloys. Reproduced with permission from A. Yamamoto et al., Thermoelectric Power Generation, 273-278 (2008). Copyright 2008 Cambridge University Press. 


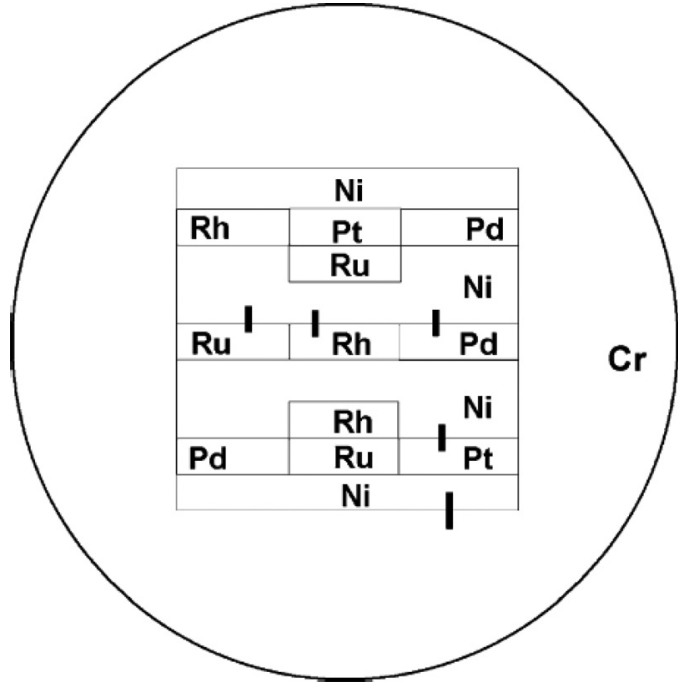

FIG. 63. Schematic diagram of the Ni-Cr-Pd-Pt-Rh-Ru diffusion multiple. The five thick bars mark locations where the composition and thermal conductivity profiles were acquired. Reproduced with permission from X. Zheng et al., Acta Mater. 55, 5177 (2007). Copyright 2007 Pergamon.

The continued demand for enhanced battery performance has led to the pursuit of improved anode and cathode materials. The primary reasons for modifying the cathode are to increase cathode capacity, address the $\mathrm{LiCoO}_{2}$ toxicity issue, and replace Co with a less expensive metal. Much of the combinatorial work to date has focused on materials such as $\mathrm{LiMnO}_{3}$ and Li-Ni-Co-Ti oxides. For the anode, the primary reason is the desire to intercalate more $\mathrm{Li}$ atoms per $\mathrm{C}$ (graphite) atom, thus increasing the total capacity of the battery, while reducing the degradation of battery performance as a function of charge/discharge cycles ("cycle fade"). Whereas $6 \mathrm{C}$ atoms are required to intercalate one $\mathrm{Li}$ atom, the same can be accomplished with only $4 \mathrm{Sn}$ atoms. ${ }^{238}$ The primary source of cycle fade for Li ion battery anodes is the large volume change that must be accommodated during $\mathrm{Li}$ intercalation, which introduces large stresses and therefore fracture of the anode, with a resultant capacity reduction. Thus, two major strategies have been pursued to preserve the capacity while mitigating cycle fade, i.e., placing the active metals in a passive matrix, and the use of amorphous materials.

The large compositional phase space represented by anodic and cathodic materials for $\mathrm{Li}$ ion batteries has motivated a number of academic and industrial groups to utilize high throughput methods to identify useful materials. Typical cathode libraries are prepared by UHV sputtering $^{238,239}$ and ink jet deposition. ${ }^{240}$ Other studies employed more traditional bulk synthesis techniques such as liquid phase precursor deposition, where libraries are created utilizing automated robots. ${ }^{241}$ Anode libraries tend to be deposited via sputtering to take advantage of the high quenching rates inherent to that technique. Screening of both anode and cathode libraries is typically done either through parallel techniques such as imaging, ${ }^{240}$ Fig. 65(a), the use of automated serial arrays, ${ }^{242-245}$ Fig. 65(b), or the use of serial electrochemical tests via automated scanning probe measurements. ${ }^{246,247}$
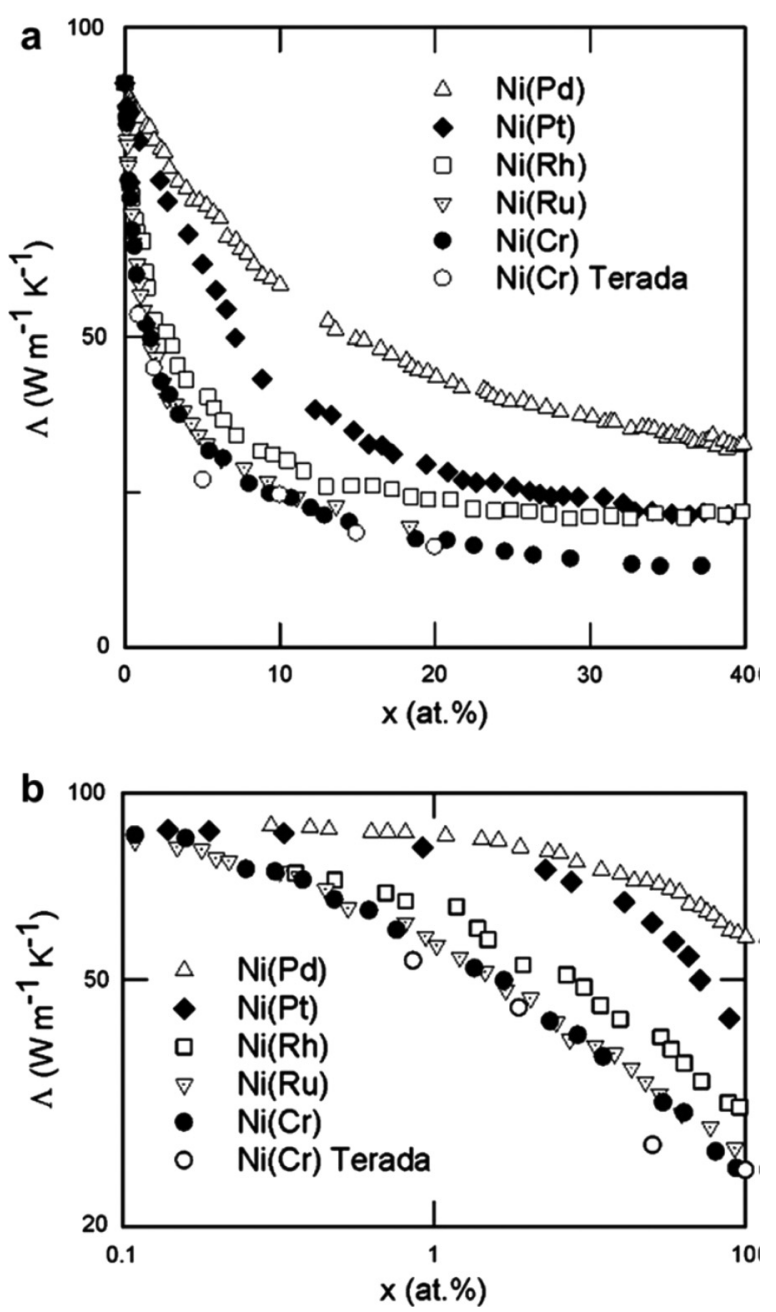

FIG. 64. (a) Composition dependence of the thermal conductivity at room temperature for $\mathrm{Ni}(\mathrm{Cr}), \mathrm{Ni}(\mathrm{Pd}), \mathrm{Ni}(\mathrm{Pt}), \mathrm{Ni}(\mathrm{Rh})$, and $\mathrm{Ni}(\mathrm{Ru})$ solid solutions. (b) Composition dependence of the thermal conductivity for solute concentrations between 0.1 and 10 at. \% plotted on a log-log scale. Open triangles, solid diamonds, open squares, dotted triangles, and solid circles are data for solutes of $\mathrm{Pd}, \mathrm{Pt}, \mathrm{Rh}, \mathrm{Ru}$, and $\mathrm{Cr}$, respectively. Open circles are for earlier non-combinatorial data reported for $\mathrm{Ni}(\mathrm{Cr})$ alloys (see Ref. 436). Reproduced with permission from X. Zheng et al., Acta Mater. 55, 5177 (2007). Copyright 2007 Pergamon.

\section{Cathode materials}

$\mathrm{LiNiO}_{2}$ is considered a potential replacement material for $\mathrm{LiCoO}_{2}$ due to its increased capacity and the lower cost of Ni. However, during cycling, $\mathrm{LiNiO}_{2}$ undergoes structural rearrangements, which eventually lead to cycle fade. It has been proposed that the cycling capabilities could be improved by the substitution of other elements for $\mathrm{Ni}$. Combinatorial computational chemistry calculations were used to investigate the structural properties of $\mathrm{LiCoO}_{2}$, $\mathrm{LiNiO}_{2}$, and $\mathrm{Li}_{0.66} \mathrm{Ni}_{0.5} \mathrm{Me}_{0.5} \mathrm{O}_{2}$, where $\mathrm{Me}=\mathrm{Cr}, \mathrm{Al}$, and Co. ${ }^{248}$ To investigate the de-intercalation of $\mathrm{Li}$ from the starting materials, structures of the form $\mathrm{Li}_{\mathrm{x}} \mathrm{MO}_{2}$ with $\mathrm{x}=1$, $0.66,0.33$, and 0 were created by removing $\mathrm{Li}$ ions and relaxing the structure. For the case of $\mathrm{LiNiO}_{2}$, as the $\mathrm{Li}$ was de-intercalated, the $\mathrm{Ni}$ ions went from a trivalent to a tetravalent state, with a substantial change in the $\mathrm{Ni}-\mathrm{O}$ bond length. It was found that with a $1: 1$ ratio of $\mathrm{Ni}$ and $\mathrm{Co}$, the $\mathrm{Co}-\mathrm{O}$ 


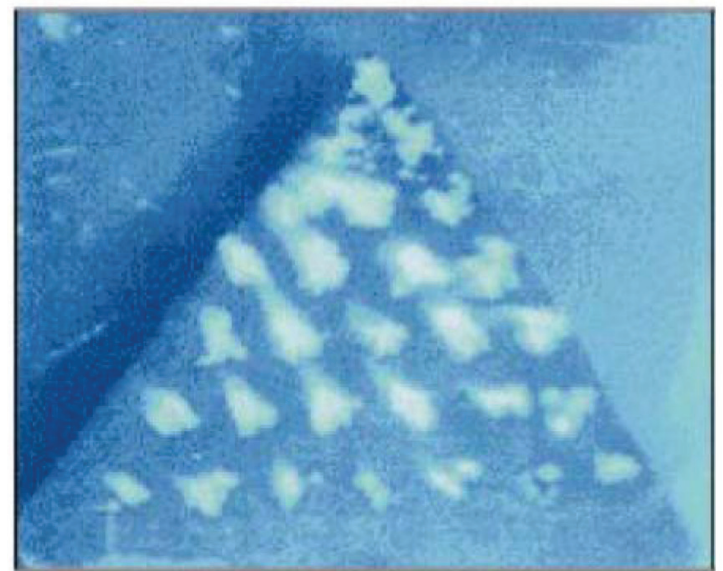

(a)

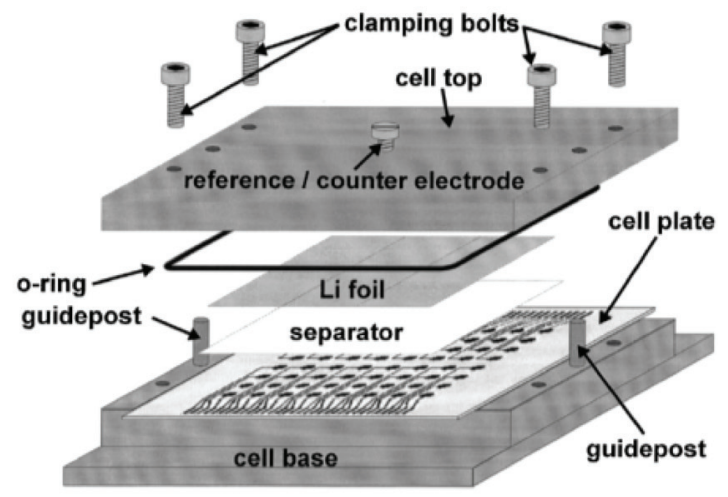

(b)

FIG. 65. Example of use of (a) fluorescence libraries. Reproduced with permission from E. Reddington et al., Science 280, 1735 (1998). Copyright 1998 American Association for the Advancement of Science. (b) Apparatus for parallel electrochemical measurements to screen battery materials. Reproduced with permission from M. D. Fleischauer et al., J. Electrochem. Soc. 150, A1465 (2003). Copyright 2003 The Electrochemical Society, Inc. bond lengths were between those of the two types of $\mathrm{Ni}$ bonds, and it was thus postulated that this could mitigate the distortion of the $\mathrm{LiNiO}_{2}$ structure during charging/ discharging.

The experimental synthesis of Li-O based cathode libraries was realized through the use of an automated solution atomization process, called the "M-ist" approach. Using an array of syringe pumps, liquid precursors were dosed into and mixed in a manifold, delivered to a robotically positioned stainless steel nozzle, and then atomized onto a substrate biased with a high voltage. With this approach hundreds of samples with diameters on the order of $3 \mathrm{~mm}$ were deposited; the total amount of material in each sample was about $0.1 \mathrm{mg} .{ }^{241}$ It has been proposed that layered oxides, or oxides with the ramsdellite structure would have superior electrode properties; therefore, initial screening of the mixed oxide materials libraries focused on scanning XRD studies. In the Li-Ni-Co ternary oxide system, a large region in the center of the phase diagram was found to consist of single phase layered oxides. In the pseudo four component system Li-Ni-Co-Ti-O, a large region of potential cathode materials with layered structures of the form $\mathrm{Li}_{\mathrm{x}}\left(\mathrm{Ni}_{\mathrm{y}} \mathrm{Co}_{\mathrm{z}} \mathrm{Ti}_{\mathrm{w}}\right) \mathrm{O}_{2}$, covering the range of compositions $(\mathrm{x} \sim 1,0<\mathrm{y}<1,0<\mathrm{z}<1$, $0<\mathrm{w}<0.2$ ) was found. ${ }^{249}$ A large region containing the ramsdellite structure was also found by substituting $\mathrm{CrO}_{1.5}$ for $\mathrm{Ni}$ in the $\mathrm{LiO}_{0.5}-\mathrm{X}-\mathrm{TiO}_{2}$ system. ${ }^{250}$

More than 25000 potential cathode material compounds were explored by a combination of high throughput modeling and data mining. ${ }^{251}$ As a result, it was suggested that oxide materials such as $\mathrm{LiZnO}_{2}$ and $\mathrm{LiAlO}_{2}$ (having the hypothetical $\alpha-\mathrm{NaFeO}_{2}$ structure) could exhibit higher intercalation voltages than $\mathrm{LiCoO}_{2}{ }^{252}$ It was also found in this work that phosphate materials generally exhibit larger $\mathrm{Li}$ insertion voltages and comparable capacities compared to their oxide counterparts, Fig. 66. Through further computational and experimental methodologies, it was shown that exchanging Co with $\mathrm{Al}$ results in a cathode material with an increased cell voltage, while reducing its overall density and

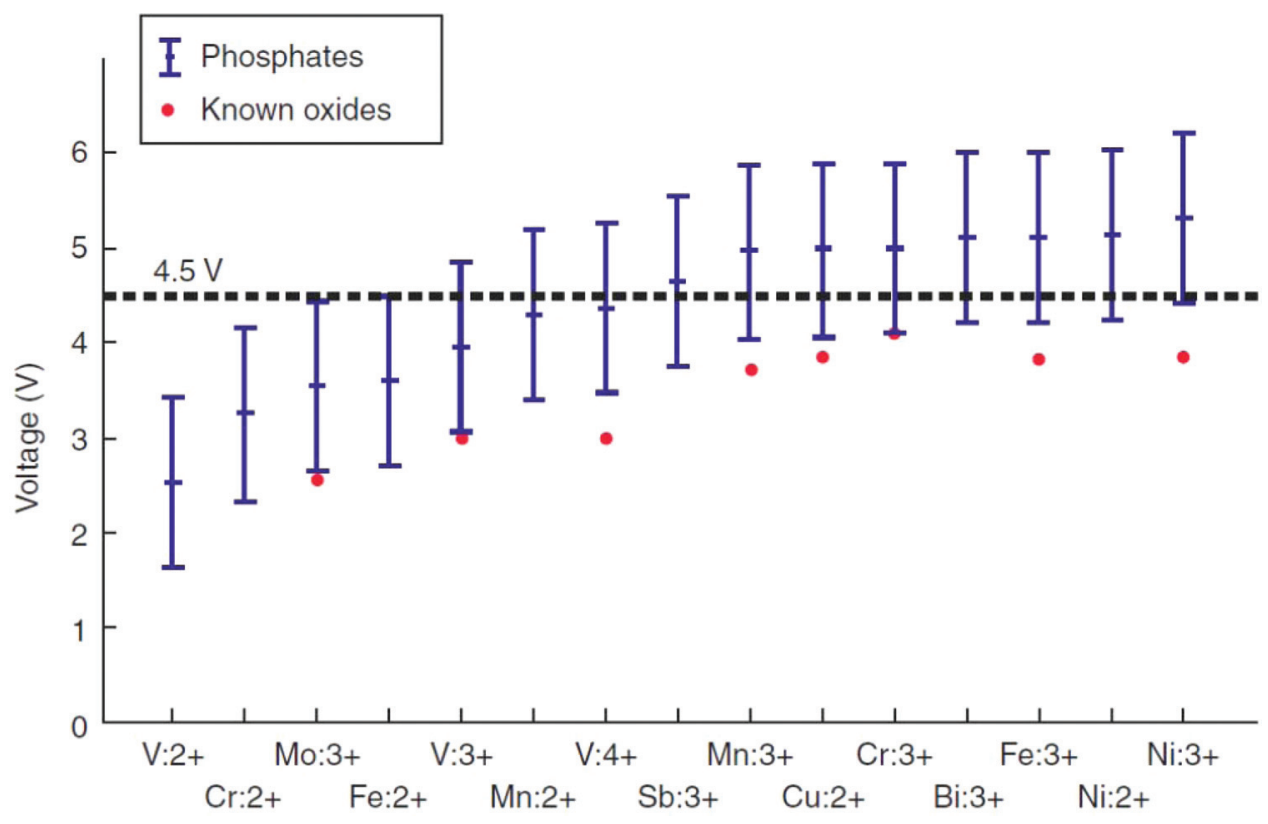

FIG. 66. Comparison of calculated range over which different cations can be oxidized by $\mathrm{Li}$ removal to voltages of known oxides. Reproduced with permission from G. Ceder, MRS Bull. 35, 693 (2010). Copyright 2010 Materials Research Society. 
cost. $^{253}$ The key computational discovery that led to this breakthrough was the finding that the oxygen atoms, not the metals, were responsible for accepting the electrons during Li intercalation. A similar approach was used to theoretically predict and experimentally verify that $\mathrm{Li}\left(\mathrm{Ni}_{0.5} \mathrm{Mn}_{0.5}\right) \mathrm{O}_{2}$ cathodes had higher rate capability than $\mathrm{LiCoO}_{2}{ }^{254}$ Further, using liquid phase reactions and post synthesis gradient annealing of arrays of cathode materials, it was demonstrated that $\mathrm{LiCo}_{33} \mathrm{Ni}_{33} \mathrm{Mn}_{.33} \mathrm{O}_{2}$ could provide competitive electrochemical performance with standard cathode materials. ${ }^{255,256}$

\section{Anode materials}

In order to increase the resistance of anodes to cycle fade, a great deal of research has been devoted to amorphous alloys containing $\mathrm{Si}$ and $\mathrm{Sn}$. The quest to identify amorphous regions of candidate phase diagrams has led to the almost exclusive use of sputtering to deposit libraries, since extremely high quench rates can be achieved. However, one difficulty of the sputtering process is subtle run-to-run compositional variations in the libraries, which can add complexity to trends observed when correlating multiple measurements taken on a series of library samples. A novel orbiting sputtering system that allows for the simultaneous deposition of up to five different samples in a single deposition, ${ }^{242,244}$ Fig. 67 has been developed to circumvent this issue. Thus, in a single deposition, libraries for XRD, SEM, gravimetric analysis (accomplished by depositing the materials directly onto an array of 25 pre-weighed Cu disks), and electrochemical cell analysis could be realized. This system was used to demonstrate the presence of a large range of amorphous compositions in the $\mathrm{Si}-\mathrm{Al}-\mathrm{Sn}$ system, for compositions containing more than $\sim 66$ at. $\% \mathrm{Si}^{257}$

A large amorphous phase region was observed in Si-AlMn libraries ${ }^{244}$ as had been observed before in rapidly cooled bulk alloys. ${ }^{258,259}$ Within this region, $\mathrm{Si}_{0.38} \mathrm{Al}_{0.52} \mathrm{Mn}_{0.10}$ was identified as exhibiting the best resistance to cycle fade, a

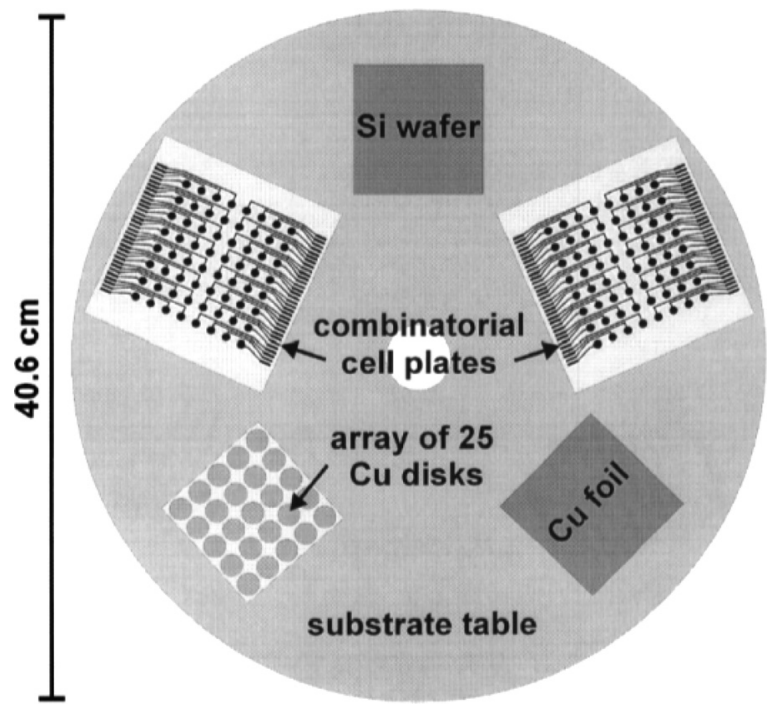

FIG. 67. Schematic of orbital sputtering system. Reproduced with permission from M. D. Fleischauer and J. R. Dahn, J. Electrochem. Soc. 151, A1216 (2004). Copyright 2004 The Electrochemical Society. gravimetric capacity superior to that of graphite, and a reversible capacity (based on standard Li battery cell type 18650) $40 \%$ higher than for graphite based cells. The predicted resistance to cycle fade was confirmed by melt spun ribbon (bulk material) experiments ${ }^{260}$ and was attributed to the formation of a supersaturated solid solution of $\mathrm{Si}$ and $\mathrm{Mn}$ in $\alpha$-Al.

A design of experiment approach was developed to facilitate discovery and optimization of useful TM additives to $\mathrm{Sn}-\mathrm{C}$ anodes, which led to the selection of the Sn-Co-C system, ${ }^{261,262}$ Fig. 68 . The experimental design first involved only selecting TMs that were known to form amorphous compounds with Sn. A second criterion was the lack of formation of TM carbides. XRD showed, for instance, that although $\mathrm{Sn}-\mathrm{Co}, \mathrm{Sn}-\mathrm{Ti}$, and $\mathrm{Sn}-\mathrm{V}$ are all amorphous for $\mathrm{Sn} / \mathrm{TM}$ ratios of 1 , the addition of small amounts of Carbon results in precipitation of the TM carbide. It was inferred that the presence of nanoparticle carbides in the $\mathrm{Ti}$ and $\mathrm{V}$ libraries facilitated the precipitation of Sn out of the amorphous matrix; while in the case of Co additives, no Sn precipitation was observed; further, for the Co case, minimal cycle fade was observed up to 27 cycles. At least two groups, working with bulk materials, have confirmed the high cycling stability of the Sn-Co-C alloys. ${ }^{263,264}$ This work has recently been extended to include the $\mathrm{Sn}-\mathrm{Si}-\mathrm{C}$ system, where the $\mathrm{C}$ serves to destabilize the two-phase regions, thus reducing the effect of volume expansion while improving cycleability. ${ }^{265}$

Sn-Cu compounds, particularly $\mathrm{Cu}_{6} \mathrm{Sn}_{5}$, have been proposed as potential crystalline anodes, but they exhibit significant cycle fade within the first 50 cycles, ${ }^{266}$ thought to be due to the large stress associated with the volume during $\mathrm{Li}$ intercalation. It has been proposed that nano-sized materials would be better suited to accommodating large volume changes. ${ }^{267}$ In a separate experiment, $\mathrm{C}$ particles that could mitigate grain growth were added, and a capacity retention in excess of $90 \%$ was observed. ${ }^{268}$ The explanation for this behavior is the formation of the $\mathrm{Cu}_{6} \mathrm{Sn}_{5}$ phase, which becomes the principal $\mathrm{Li}$ intercalation material within a $\mathrm{C}$ matrix, and prevents grain aggregation.

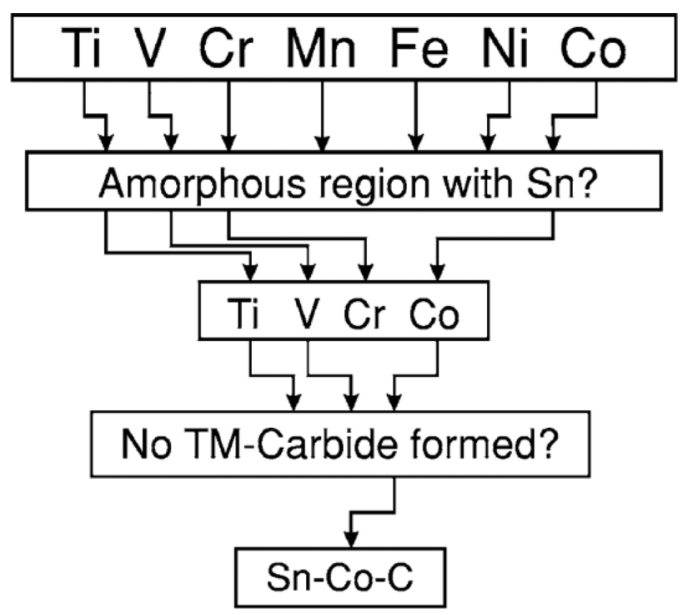

FIG. 68. Roadmap to the selection of the $\mathrm{Sn}-\mathrm{Co}-\mathrm{C}$ system as the best choice among Sn-M-C systems for negative electrodes in $\mathrm{Li}$-ion batteries. Reproduced with permission from A. D. W. Todd et al., J. Electrochem. Soc. 154, A597 (2007). Copyright 2007 The Electrochemical Society. 


\section{Hydrogen storage materials}

The penetration of fuel cell powered vehicles into the marketplace is contingent upon the ability to store substantial amounts of hydrogen within existing chassis designs. ${ }^{269,270}$ Although hydrogen may be stored either under high pressure as a gas, cryogenically as a liquid, or in the solid state as a hydride, the latter option offers the best theoretical combination of gravimetric and volumetric capacity, stability, and safety. Due to its low density, hydrogen gas must be stored at pressures as high as $70 \mathrm{MPa}$ for a three hundred mile range vehicle. Cryo-compressed hydrogen systems can provide sufficiently large gravimetric and volumetric densities of hydrogen, although substantial losses of the liquid fuel due to imperfect insulation will occur. On the other hand, hydrogen can be stored as the hydride of light elements, such as $\mathrm{Li}, \mathrm{B}, \mathrm{Mg}, \mathrm{N}, \mathrm{Al}$, and $\mathrm{Na}$, and hydrogen concentrations as high as 18 wt. $\%$ may be achieved in some hydrides. ${ }^{271}$ The volumetric density of gaseous hydrogen at RT and 1 bar is $8.2 \times 10^{-5} \mathrm{~kg} / \mathrm{l}$, while metal hydrides such as $\mathrm{LiBH}_{4}$ can store as much as $1.2 \times 10^{-1} \mathrm{~kg} / \mathrm{l}$. Even at $70 \mathrm{MPa}$, the volumetric density of gaseous hydrogen is $5.7 \times 10^{-2} \mathrm{~kg} / \mathrm{l}$, approximately half that of $\mathrm{LiBH}_{4}$. The efficient renewable production of sufficiently pure hydrogen represents a significant challenge that must be addressed in conjunction with the search for suitable hydride materials. High-throughput efforts in the field of solar energy splitting of water have been developed by several groups, ${ }^{272-274}$ and a recent critical review on this subject has been published. ${ }^{275}$

Despite these advantages, the implementation of metal and chemical hydrides has been hindered by the dehydrogenation thermodynamics and kinetics of typical materials. A large portion of the initial effort of the US Department of Energy (DOE) sponsored metal hydride work focused on studying known hydrides, with or without the addition of TM based oxides and chlorides, to expedite kinetics. ${ }^{276}$ First, the addition of $\mathrm{TiCl}_{3}$ to $\mathrm{NaAlH}_{4}$ had been shown to substantially change its thermodynamics and kinetics and thus an entire community formed around the deliberate doping of known hydrides. ${ }^{277,278}$ Second, the dehydrogenation mechanisms of the existing materials had not been well studied and extensive work was needed to gain a basic understanding of the chemical mechanisms involved. Finally, it was thought that mixtures of thermodynamically stable complex hydrides would not form single phases, but rather multiphase mixtures.

The landscape of the hydrogen storage community was significantly altered by the reassessment of some early research $^{279}$ that demonstrated the enthalpy of formation for $\mathrm{MgH}_{2}$ could be lowered from $76 \mathrm{~kJ} / \mathrm{mol} \mathrm{H}$ to $63 \mathrm{~kJ} / \mathrm{mol} \mathrm{H}$ by alloying with $\mathrm{Ni}$. Thus, a niche for combinatorial materials science was formed to discover rapid methods of screening additives to metal hydrides based on their efficacy in promoting hydrogenation/dehydrogenation kinetics. More recently, it was shown that a 1:1 mixture of $\mathrm{LiBH}_{4}$ and $\mathrm{MgH}_{2}$ resulted in a substantial reduction in the enthalpy of formation of pure $\mathrm{LiBH}_{4}$, from $90 \mathrm{~kJ} / \mathrm{mol} \mathrm{H}$ to $50 \mathrm{~kJ} / \mathrm{mol}$ $\mathrm{H}_{2}{ }^{280,281}$ Experiments involving mixing of other complex hydrides, such as $\mathrm{LiNH}_{2}$ with $\mathrm{MgH}_{2}$ or $\mathrm{LiH}$, or $\mathrm{LiBH}_{4}$ and $\mathrm{LiNH}_{2}$ soon followed. ${ }^{282-286}$ Surprisingly, in 1:1 mixtures of
$\mathrm{LiNH}_{2}$ and $\mathrm{MgH}_{2}$, it has since been demonstrated that after only one dehydrogenation/hydrogenation cycle, no $\mathrm{LiNH}_{2}$ remains in the powder; subsequent hydrogen cycling involves only the compounds $\mathrm{Mg}\left(\mathrm{NH}_{2}\right)_{2}, \mathrm{LiH}$, and $\mathrm{MgH}_{2}{ }^{287}$ Further, it has been shown experimentally that the initial ratio of $\mathrm{LiNH}_{2}$ to $\mathrm{MgH}_{2}$ can affect the overall reaction mechanism. ${ }^{288,289}$ For the case of $\mathrm{LiBH}_{4}$ and $\mathrm{LiNH}_{2}$ mixtures, it was surprisingly discovered that almost complete solubility between the two phases could be achieved, with free interchange of the $\mathrm{NH}_{2}$ and $\mathrm{BH}_{4}$ anions. ${ }^{290}$ Investigations into ternary, quaternary, and higher order mixtures were found to yield still more interesting materials with previously uninvestigated chemistries. ${ }^{291-293}$

The multidimensional compositional space of importance to the hydrogen storage community, encompassing cation ( $\mathrm{Li}$, $\mathrm{Mg}, \mathrm{Na}, \mathrm{Ti}, \mathrm{K}$, etc. $)$ and anion substitutions $\left(\mathrm{AlH}_{4}, \mathrm{BH}_{4}, \mathrm{NH}_{2}\right.$, etc.), catalyst composition $\left(\mathrm{MgH}_{2}, \mathrm{TiCl}_{3}\right.$, etc.), nanostructure, thermodynamics, and kinetics is inaccessible, from a practical standpoint, using traditional materials science techniques. Therefore, the hydrogen storage community has embraced high throughput methodologies to enhance the probability of discovering breakthrough materials. Their efforts have spanned the spectrum from database searches and first principles DFT calculations, to the demonstration of high throughput thin film measurement techniques; the latter are especially useful for experimental verification of predictive modeling results.

What follows in this section is divided into three subsections: data mining and modeling, used to rapidly screen millions of possible reactions for the relatively small number that are theoretically possible; thin film combinatorial experiments, aimed at developing a comprehensive high throughput scheme that enable working with nanogram to microgram quantities of material; and combinatorial powder studies, which mimic the bulk materials that would actually be used in a hydrogen storage system.

\section{Data mining and modeling}

The advent and general availability of DFT software packages such as Vienna Ab Initio Simulation (VASP), ${ }^{294}$ as well as large scale tabulations of experimentally obtained crystal structure databases, ${ }^{295-297}$ have enabled computational screening of millions of possible hydrogenation/dehydrogenation reactions. These methods provide a powerful means of searching an enormous phase space in a short (days) time span. For example, more than one hundred dehydrogenation reactions involving 49 compounds were computationally screened, ${ }^{298}$ Fig. 69. From these reactions, five were identified with reaction enthalpies that would allow the release of hydrogen at pressures and temperatures relevant to vehicular applications. Through detailed comparison of their calculated reaction enthalpies and values available through the literature, the authors determined that their error bar was on the order of $\pm 10 \mathrm{~kJ} /$ mole $\mathrm{H}_{2},{ }^{299}$ however, depending upon the system chosen, this error can correspond to a variation in predicted equilibrium pressure of two orders of magnitude. An extremely comprehensive screening of $16 \times 10^{6}$ reactions ${ }^{300,301}$ was also carried out; it is worth noting that in this case, only 44 candidate reactions were identified. 


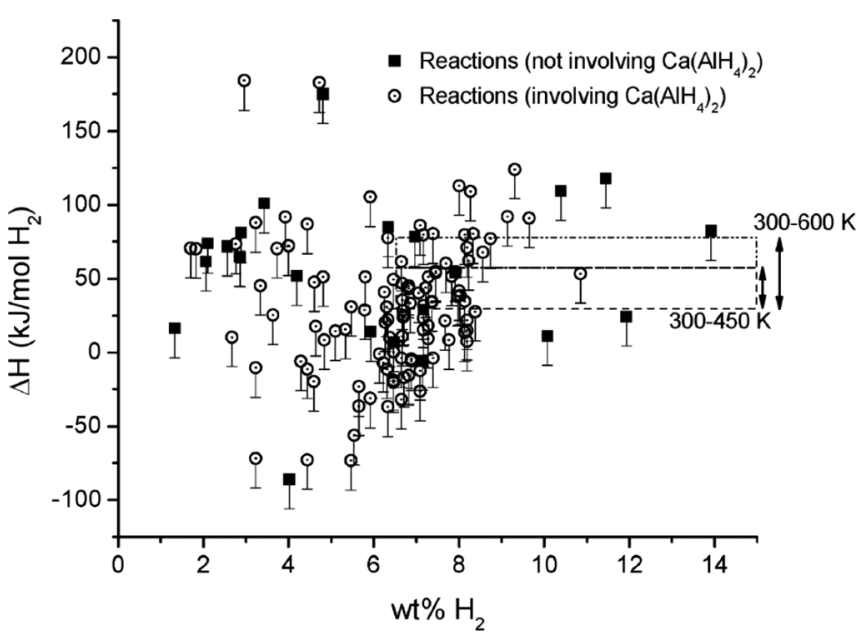

FIG. 69. DFT calculated enthalpies of formation for a large number of possible one step hydrogen release reactions. The reactions that fall within the bounds of the dotted lines were identified as being promising enough to merit experimental investigation. Reproduced with permission from S. V. Alapati et al., J. Phys. Chem. C 111, 1584 (2007). Copyright 2007 American Chemical Society.

A similar methodology was used to investigate destabilization of $\mathrm{LiBH}_{4}$ and $\mathrm{Ca}\left(\mathrm{BH}_{4}\right)_{2}$ by $\mathrm{TMs}$ and simple metal hydrides. ${ }^{280}$ Twenty candidate reactions were investigated, and it was found that additives such as $\mathrm{Cr}$ and $\mathrm{ScH}_{2}$ could sufficiently destabilize $\mathrm{Ca}\left(\mathrm{BH}_{4}\right)_{2}$ to the temperature and pressure requirements for vehicular applications. Further, prior to this work, it was assumed that borohydrides such as $\mathrm{LiBH}_{4}$ decomposed into a mixture of $\mathrm{Li}, \mathrm{B}$, and $\mathrm{H}_{2}$. Subsequent work demonstrated that an entire series of intermediate compounds, such as $\mathrm{Li}_{2} \mathrm{~B}_{12} \mathrm{H}_{12}$ and $\mathrm{CaB}_{12} \mathrm{H}_{12}$ are formed during hydrogen cycling of many borohydrides. ${ }^{302,303}$ The effect of destabilizers on the reaction paths of these newly discovered reaction products were also studied, ${ }^{304}$ where it was found that the intermediate phases were very stable, with enthalpies greater than $70 \mathrm{~kJ} / \mathrm{mol} \mathrm{H}_{2}$, but could be substantially destabilized in the presence of basic metal hydrides such as $\mathrm{MgH}_{2}$. In order to perform DFT calculations for $\mathrm{M}\left(\mathrm{B}_{12} \mathrm{H}_{12}\right)$ materials, their structure must be calculated. $^{305}$

Further work in the simulation and modeling area was directed towards reaction pathways, enthalpies, and hydrogen release in the $\mathrm{MgH}_{2}-\mathrm{LiH}-\mathrm{NH}_{3}$ ternary system, Fig. $70 .^{306}$ An outcome of another simulation was the first report of some phase structures in the $\mathrm{Li}-\mathrm{Mg}-\mathrm{Al}-\mathrm{H}$ system, e.g., the unstable $\mathrm{CaGaF}_{5}$-type structure of $\operatorname{LiMg}\left(\mathrm{AlH}_{4}\right)_{3}$; here, it was determined that $\operatorname{LiMg}\left(\mathrm{AlH}_{4}\right)_{3}$ decomposes to $\mathrm{LiMgAlH}_{6}$ at $\mathrm{RT}^{307}$ Recent interest in ammonia borane $\left(\mathrm{NH}_{3} \mathrm{BH}_{3}\right)$ and its intermediate decomposition products spurred calculations of the structure and reaction enthalpies of $\left(\mathrm{NH}_{4}\right)_{2} \mathrm{~B}_{12} \mathrm{H}_{12}$. ${ }^{308}$

\section{Thin film combinatorial work}

The goal of any hydrogen storage program is to manufacture materials on the kilogram scale. However, a number of groups have utilized high throughput thin film methodologies to screen hydrogen storage materials on the microgram scale $^{308-311}$ and have been able to determine the phase diagram of a hydride system on a single library sample. Also, thin films can mitigate the role of kinetics in screening hydride samples; the films can be grown with arbitrarily small grain sizes, allowing for rapid cycling of materials, or the effective grain size can be deliberately modulated to determine the presence of rate limiting steps during hydrogenation. In thin film experiments, the compositional resolution is generally limited by the size of the sample and the
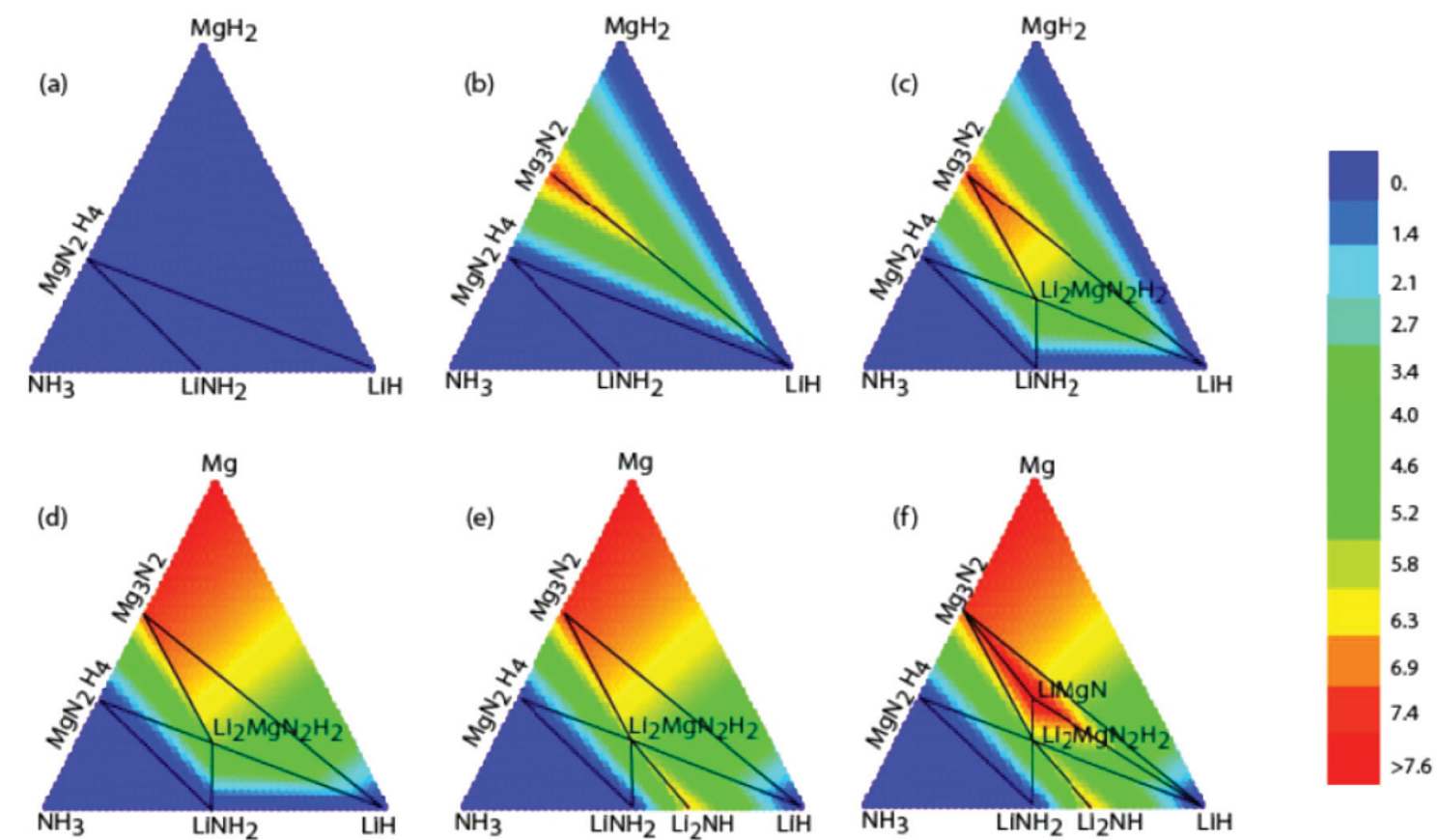

FIG. 70. Calculated hydrogen release (in wt. \%) across the $\mathrm{Li}-\mathrm{Mg}-\mathrm{N}-\mathrm{H}$ system as a function of temperature. Each figure corresponds to a specific temperature range (a) $\mathrm{T}<30 \mathrm{~K}$, (b) $130 \mathrm{~K}<\mathrm{T}$, $426 \mathrm{~K}$, (c) $426 \mathrm{~K}<\mathrm{T}<458 \mathrm{~K}$, (d) $458 \mathrm{~K}<\mathrm{T}<606 \mathrm{~K}$, (e) $606 \mathrm{~K}<\mathrm{T}<734 \mathrm{~K}$, and (f) $743 \mathrm{~K}<\mathrm{T}<780 \mathrm{~K}$. Reproduced with permission from C. Wolverton et al., J. Phys. Condens. Matter 20, 064228 (2008). Copyright 2008 Institute of Physics. 
spatial resolution/sampling volume requirements of the measurement system. The number of samples to be characterized from a given library can vary from 100 to $1000 .^{311-313}$

However, there are significant challenges to the thin film approach. For example, a phase that can be synthesized through thin film deposition techniques may not be capable of being produced in kilogram quantities using traditional bulk synthesis techniques. Also, in thin film form, the influences of crystallographic texture and film stress may alter the thermodynamics of the materials being investigated. Finally, in thin films, measurements of hydrogen storage properties cannot be obtained using traditional volumetric and gravimetric measurement techniques. To circumvent this issue, the combinatorial community has utilized a series of indirect measurement techniques, such as film transparency, IR emissivity, XRD, and cantilever deflection to characterize thin film libraries. ${ }^{310,314-316}$ Using these tools, combinatorial thin film studies can provide an important bridge between theoretical and bulk powder work. Thus, thin film demonstration of a predicted reaction pathway, with an enthalpy close to the expected value, can provide evidence that such a reaction is not kinetically limited. In the bulk powder case, demonstrating that a given reaction does not occur, but rather decomposition proceeds through a different reaction path can help theorists refine their models and add new compounds to their databases for subsequent studies.

Many materials, such as Mg and $\mathrm{Y}$, undergo a metallic to insulator transition upon exposure to hydrogen. ${ }^{317}$ The consequent increase in resistivity results in an increase in the amount of IR radiation by the part of the library sample that has absorbed hydrogen. IR emissivity, Fig. 71, was used to screen for hydrogen absorption temperatures in thin film libraries of $\mathrm{Mg}-\mathrm{Ni}-\mathrm{Fe},{ }^{318} \mathrm{Fig}$. 72. In a subsequent study, IR emissivity mapping was used in tandem with TEM to study hydrogenation in Mg-Ni libraries. ${ }^{319}$ They found that over a broad range of $\mathrm{Ni}$ concentrations, a $\mathrm{Ni}$ stabilized fcc $\mathrm{Mg}$

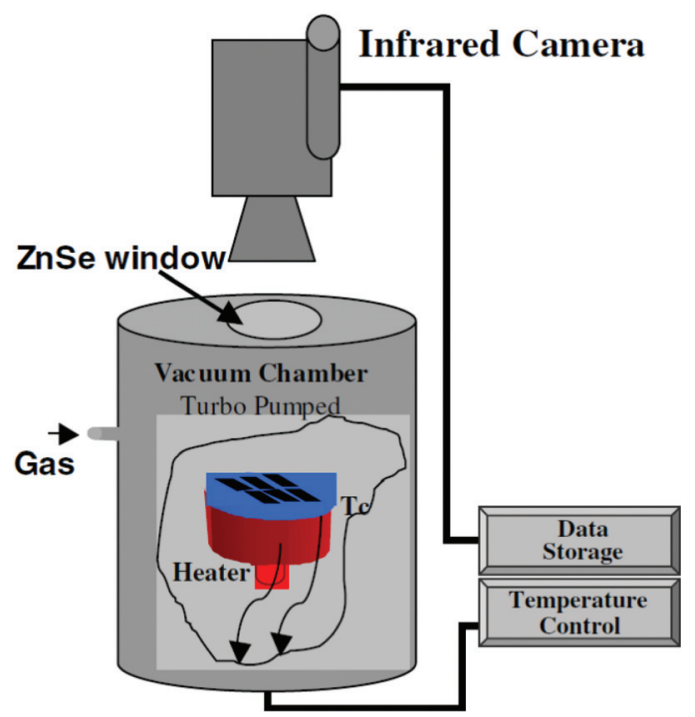

FIG. 71. Cartoon of a typical IR emissivity measurement system for thin film hydride characterization. Reprinted with permission from C. H. Olk et al., J. Appl. Phys. 94, 720 (2003). Copyright 2003 American Institute of Physics. phase coexisted with precipitates of $\mathrm{Mg}_{2} \mathrm{Ni}$ and $\mathrm{MgNi}_{2}$, which resulted in an increased hydrogenation pressure. It was further demonstrated that this technique could be used to monitor metal to semiconductor, as well as metal to metal transformations; this finding is important since many hydrogen storage materials can exhibit multiple decomposition steps, and not all of the products are insulating. ${ }^{313,320}$

CCS libraries of hydrogen storage materials enable measurement of the thermodynamic properties, but to determine reaction kinetics, it is useful to employ thickness wedge libraries, where the importance of grain size on the reaction rates can be studied. Materials such as $\mathrm{MgH}_{2}$ strongly inhibit the diffusion of hydrogen, and thus present a substantial barrier to efficient hydrogenation. The wedge library technique was used to great advantage ${ }^{321}$ to demonstrate that for a $\mathrm{Mg}_{98.4} \mathrm{Ti}_{1.6}$ film the $\mathrm{MgH}_{2}$ layer grows quickly $(0.3 \mathrm{~nm} / \mathrm{s})$, until it is about $150 \mathrm{~nm}$ thick. At this stage, the hydride growth rate drops by an order of magnitude; thus, the optimal particle size for the bulk powder alloy would be in the range of $300 \mathrm{~nm}$, since hydride formation in the bulk would progress by radial hydrogen diffusion. Thus, the information obtained from thickness gradient libraries can provide the manufacturers of the bulk powder alloys with important particle size information.

The measurement of the change in optical transmissivity in thin film libraries due to hydrogen reaction has been coined "hydrogenography" and has proved to be a robust screening technique. ${ }^{316,322}$ In a two phase mixture of a metal and its hydride, the concentration of hydrogen is proportional to the log of the optical transmission. ${ }^{323}$ Thus, by measuring the change in transmission as a function of pressure, the enthalpy of hydride formation for a thin film can be directly measured. Much of the hydrogenography work has been reviewed. ${ }^{324}$ Similar to IR emissivity, hydrogenography can also detect metal to metal transitions during hydrogenation of films; ${ }^{325}$ this feature makes hydrogenography useful for studying other classes of materials, such as hydrogen separation membranes. ${ }^{326}$ Hydrogenography has also been utilized as a switchable mirror technology, taking advantage of the change in transmissivity that results from hydrogenation, as well as other applications. ${ }^{327-330}$

Hydrogenography has been applied for compositional mapping of a number of $\mathrm{Mg}$ based systems such as $\mathrm{Mg}-\mathrm{Ni},{ }^{309,331} \mathrm{Mg}-\mathrm{Ni}-\mathrm{Ti},{ }^{332} \mathrm{Mg}-\mathrm{Al},{ }^{333}$ and $\mathrm{Mg}-\mathrm{Al}-\mathrm{Ti}^{334} \mathrm{~A}$ notable result from this body of work was the discovery ${ }^{316}$ of the alloy $\mathrm{Mg}_{69} \mathrm{Ni}_{26} \mathrm{Ti}_{5}$, Fig. 73, which has an hydrogenation enthalpy of $-39.2 \mathrm{~kJ} / \mathrm{mol} \mathrm{H}_{2}$, quite close to the ideal value of $-40 \mathrm{~kJ} / \mathrm{mol} \mathrm{H}_{2}$ for on board vehicular hydrogen storage. A subsequent DFT study validated the experimentally measured enthalpy and demonstrated that in the hydrogenated state the alloy existed as a Ti-doped $\mathrm{Mg}_{2} \mathrm{NiH}_{4}$ alloy, which upon dehydrogenation decomposes to a mixture of $\mathrm{Mg}_{2} \mathrm{Ni}$ and a $\mathrm{Ti}-\mathrm{Ni}$ intermetallic phase. In a related study, it was found that using a Ti buffer layer in $\mathrm{Mg}-\mathrm{Al}$ films facilitated the hydrogenation of $\mathrm{Mg}\left(\mathrm{AlH}_{4}\right)_{2}$ below 1 bar. Unfortunately, knowledge of such catalytic behavior has not been able to inform the practice of using milling to dope bulk powder lots.

Other approaches for investigating thin film hydrogen storage materials libraries include XRD, prompt gamma 


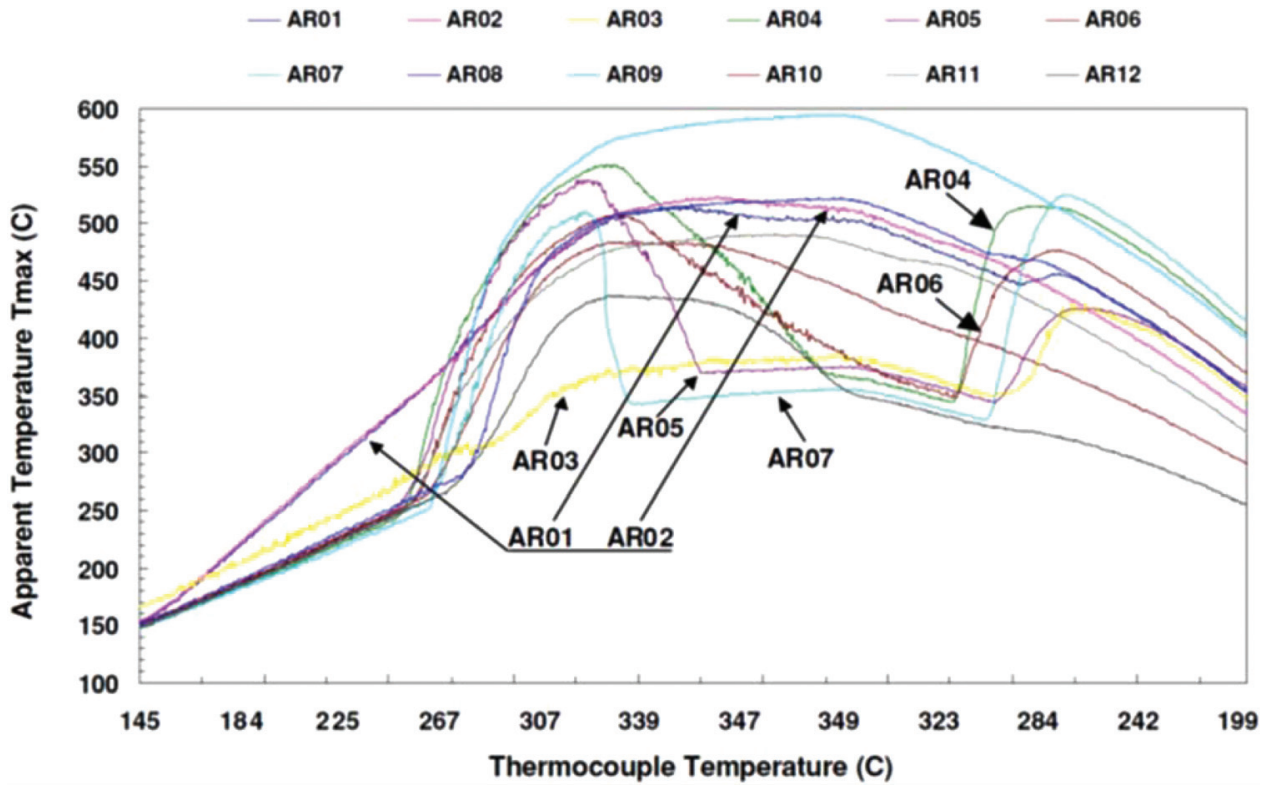

FIG. 72. Representative IR emissivity data from a $\mathrm{Mg}-\mathrm{Ni}$ composition spread. Reprinted with permission from C. $\mathrm{H}$. Olk et al., J. Appl. Phys. 94, 720 (2003). Copyright 2003 American Institute of Physics.

activation analysis (PGAA), and RBS. ${ }^{314,335,336}$ Although these methods are useful, there are disadvantages to each. In the case of PGAA and RBS large, expensive and dedicated equipment are required. XRD has limited usefulness because many hydrogen storage materials, e.g., borohydrides, tend to decompose into amorphous phases. Another intriguing approach to combinatorial screening of hydrides is the measurement of microelectromechanical (MEMS) cantilever displacement, which results from the expansion/contraction of the material as it is cycled. ${ }^{310,337}$ Thus, far this technique has only been applied to simple Mg hydrides.

\section{Combinatorial studies of hydride powders}

The goal of high throughput experimentation on powder samples is to demonstrate their properties on the gram to
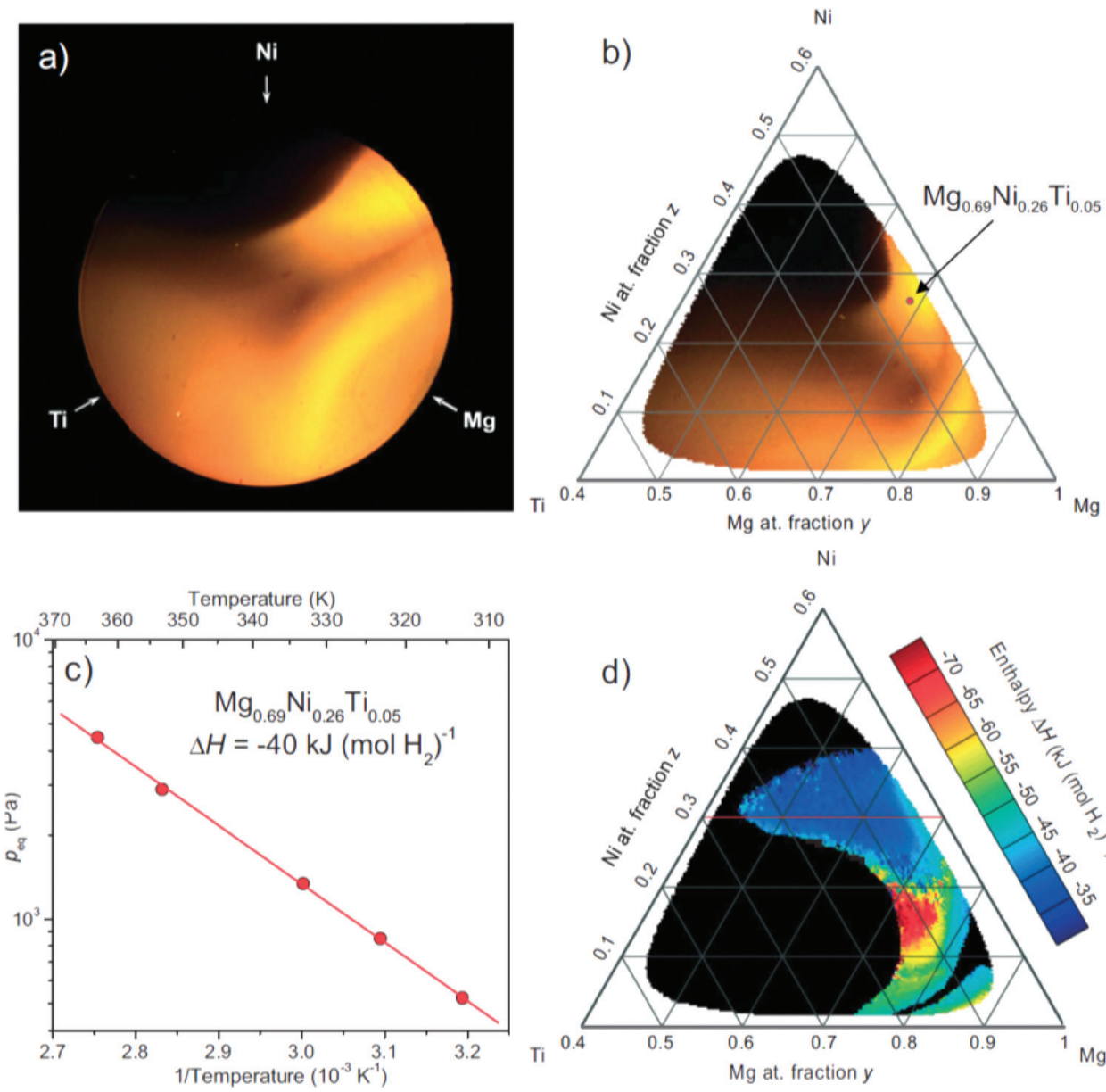

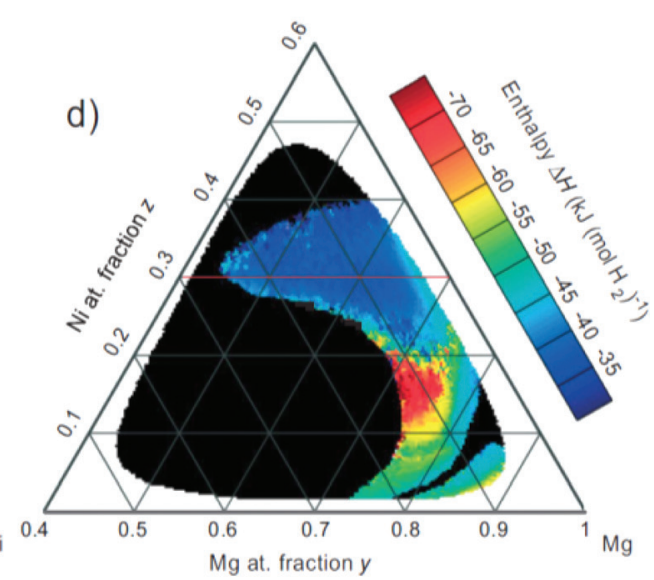

FIG. 73. Representative data taken via hydrogenography (a) an image of a backlit combinatorial sample is obtained at a set temperature and pressure, (b) the transmission of each point is mapped to the ternary phase diagram, (c) the plateau pressure of each composition is evaluated as a function of temperature, and (d) the enthalpy is plotted on the phase diagram. Reproduced with permission from R. Gremaud et al., Adv. Mater. 19, 2813 (2007). Copyright 2007 Wiley-VCH Verlag Gmbh \& Co. 
kilogram scale. In high throughput ball milling and characterization experiments, the number of different material samples is limited to the number of milling jars. The price of such systems rapidly increases with the number of samples to be investigated per experiment. Typical bulk powder synthesis and processing involves physical mixing, via ball milling, of base materials (e.g., $\mathrm{LiBH}_{4}$ ), with a destabilizing material to alter its thermodynamics (e.g., $\mathrm{MgH}_{2}$ ), and finally a catalyst to promote kinetics (e.g., $\mathrm{TiCl}_{3}$ ). The ball to powder ratio, milling speed (energy), ball composition, and order in which the components are milled can dramatically affect their hydrogen storage properties. For example, in the ternary $\mathrm{LiBH}_{4}-\mathrm{LiNH}_{2}-\mathrm{MgH}_{2}$ system, the overall hydrogen reaction pathways can be altered depending upon whether all components are milled simultaneously in a single jar, or are first milled individually to achieve smaller starting grain sizes and then milled together. ${ }^{291,293}$

Several industrial scale high throughput efforts have been implemented to screen for powder materials, using parallel ball milling apparatus. ${ }^{338,339}$ These systems can synthesize and measure the pressure-composition-isotherm characteristics of up to forty-eight individual powder samples at a time. In a typical experiment, a sample of known mass is held in a vessel of known volume at a constant temperature. As the pressure is increased in discrete increments, the change in the system pressure due to hydrogen absorption/desorption is measured. Using this methodology, a self-catalyzing hydride system, $\mathrm{LiNH}_{2}-\mathrm{LiBH}_{4}-\mathrm{MgH}_{2}$, ${ }^{338}$ was discovered, Fig. 74. The optimal combination of low temperature hydrogen release $\left(<180^{\circ} \mathrm{C}\right)$, weight percent hydrogen released (less than $4 \mathrm{wt}$. \%), and reversibility were observed at ratios of 2:1:1 and 2:0.5:1 in this system. ${ }^{340}$ An interesting aspect of work on this ternary system was the comprehensive use of both experiment and theory to first identify promising systems, followed by larger scale powder experiments.

For most hydrogen containing compounds, vibrational spectroscopy can provide important structural and local binding site information about the material; such information is complementary to gravimetric or volumetric measurements. In-situ high throughput spectroscopy has increasingly become a part of the array of tools used by the hydrogen storage community. ${ }^{312,341,342}$ It has been applied to discrete powder arrays as well as to diffusion couple samples. Micro-Raman spectroscopy was used to measure the diffusion of atomic and molecular hydrogen in the borohydride cluster, ${ }^{341,342}$ and it was demonstrated, by radioisotopic substitution, that hydrogen was transported almost exclusively by the diffusion of $\mathrm{BH}_{4}{ }^{-}$, the exchange of single hydrogen ions occurring at a rate ten orders of magnitude slower.

\section{Catalysts for fuel cell anodes}

Fuel cells, which convert chemical energy into electrical energy, have long been considered a potential device for both mobile and stationary energy generation applications. ${ }^{343}$ In the most basic case, hydrogen and oxygen react to form water, and electrical energy is generated. However, fuel cells can run on fuels other than hydrogen, as is the case illustrated in Fig. 75, in which a mixture of methanol and water impinge on the anode of the cell, and are electrochemically reformed and reduced, respectively, into $\mathrm{H}_{2}$ gas and $\mathrm{CO}_{2}$. The proton is conducted through an electrically insulating layer, while the electron is conducted through an electrical circuit, creating a current. Finally, at the cathode, molecular oxygen is reduced and combined with the proton and electron to form water. Carbonaceous fuels such as methanol $\left(\mathrm{CH}_{3} \mathrm{OH}\right)$ can serve as alternatives to molecular hydrogen; these fuels will result in the formation of $\mathrm{H}_{2}$ and $\mathrm{CO}_{2}$. The advantage of alternate fuels is that the transportation infrastructure has a century of experience in handling liquid fuels, and virtually none with hydrogen. Additionally, many sources of hydrogen are available, from methane $\left(\mathrm{CH}_{4}\right)$ to more complex hydrocarbon fuels. Much effort has been directed to finding appropriate catalysts for solid oxide fuel cells (SOFCs) and direct methanol fuel cells (DMFCs). ${ }^{24,344-347}$ However, oxidation of carbonaceous fuels takes place in multiple steps, generally including $\mathrm{C}-\mathrm{H}$ bond activation, C-O oxidation, and water adsorption, and no single element catalyst has thus far shown ideal activity and selectivity. The focus of a large portion of the combinatorial work done in this field has been on the identification of novel cathodes and anodes for SOFC and DMFC applications; however, concerns over the oxidation of carbon supports in polymer electrolyte membrane (PEM) fuel cells has led to some combinatorial work in that field. ${ }^{348}$

Several high throughput approaches to fuel cell anode catalyst discovery and optimization have been pursued. One method uses sputtering to create CCS libraries. ${ }^{349-352}$ Ink jet based solution synthesis techniques, in which metal salts are solution deposited onto an electrode, have also been employed. $^{353-355}$ The arrays are then reduced to the metallic state, frequently by borohydrides such as $\mathrm{NaBH}_{4}$. One concern with the uniform usage of $\mathrm{NaBH}_{4}$ as a reducing agent is its inability to provide the high surface area catalysts eventually required for implementation. ${ }^{355}$ Another technique is
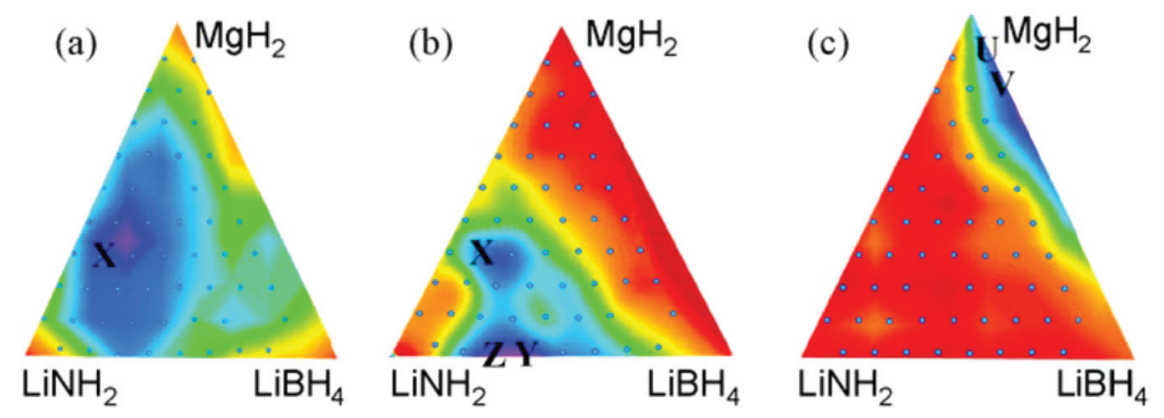

FIG. 74. Weight $\%$ hydrogen desorbed from different mixtures of $\mathrm{LiNH}_{2}-\mathrm{LiBH}_{4}-\mathrm{MgH}_{2}$ as a function of cycle and temperature. (a) First cycle at $220^{\circ} \mathrm{C}$, (b) second cycle at $285^{\circ} \mathrm{C}$, and (c) fourth cycle $350^{\circ} \mathrm{C}$. Reproduced with permission from G. J. Lewis et al., J. Alloys Compd. 446, 355 (2007). Copyright 2007 Elsevier BV. 


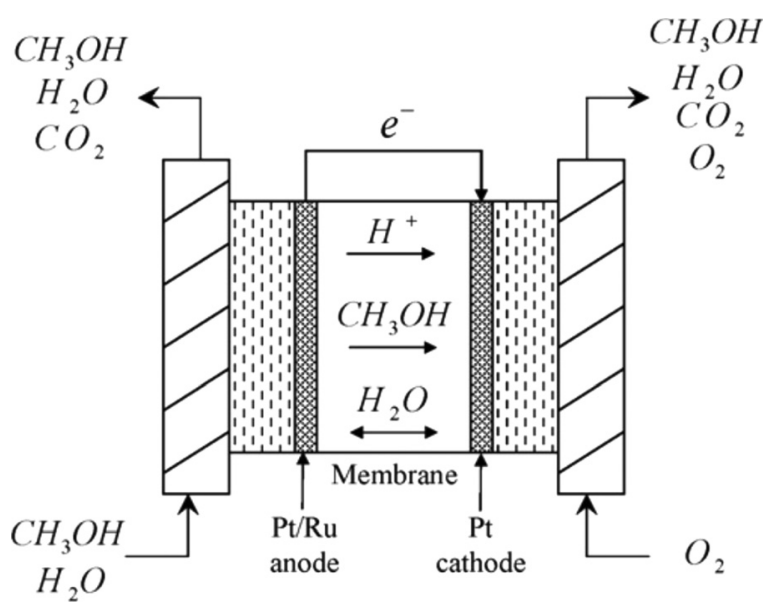

FIG. 75. Schematic of a single fuel cell (see Ref. 437). Reproduced with permission from S. Eccarius et al., J. Power Sources 179, 723 (2008). Copyright 2008 Elsevier S.A.

wet impregnation, followed by calcination at high temperatures to promote oxidation and mixing. ${ }^{356,357}$ Other groups employ electrodeposition, in which a fluid containing the catalyst precursors is pipetted onto an array of electrodes, followed by the electrodeposion of the catalyst on the electrode surface using a potentionstat, ${ }^{358}$ Fig. 76. In subsequent work, gel transfer technology was used in combination with electrodeposition to design high density CCS libraries. ${ }^{359} \mathrm{In}$ this method, a gel containing an uniform concentration of a single metal salt is impregnated at a single point with a second metal salt; the diffusion of the point source into the semi-infinite gel then creates the desired composition gradient. Samples are then electrodeposited using normal methods and the gel is removed, yielding samples with continuously varying composition gradients.

Electrochemical interrogation of the catalyst libraries is accomplished by either qualitative screening for the C-O oxidation potential or quantitative measurements of catalytic activity by monitoring the current as a function of voltage. A popular characterization method for DMFC anodes is fluorescence. ${ }^{240}$ In this technique, the library is deposited onto a conductive substrate, which acts as a common electrode for each sample. The entire sample is then immersed in a solution of quinine and methanol, and the voltage is slowly raised. When the methanol is oxidized, it releases up to 4 protons, which are absorbed by the quinine, causing it to fluoresce. This technique has been widely used as a primary screen for anodes, although it suffers from some disadvantages; it is qualitative, and offers poor spatial resolution due to diffusion of the activated quinine through the liquid. ${ }^{167,353,354,360-362}$ To mitigate diffusion issues, some groups have created semi-discrete arrays by micromachining. ${ }^{349}$ Another method of circumventing the limited spatial resolution of fluorescence is to raster a small counter electrode, which acts as a scanning electrochemical microscope ${ }^{359,363}$ because areas of the library with increased activity will generate more hydrogen and thus a larger current. Similar electrochemical screening techniques have been employed to characterize the effect of corrosion inhibiting additives to the electrolyte on different metallic wires, to great effect. ${ }^{364-366}$
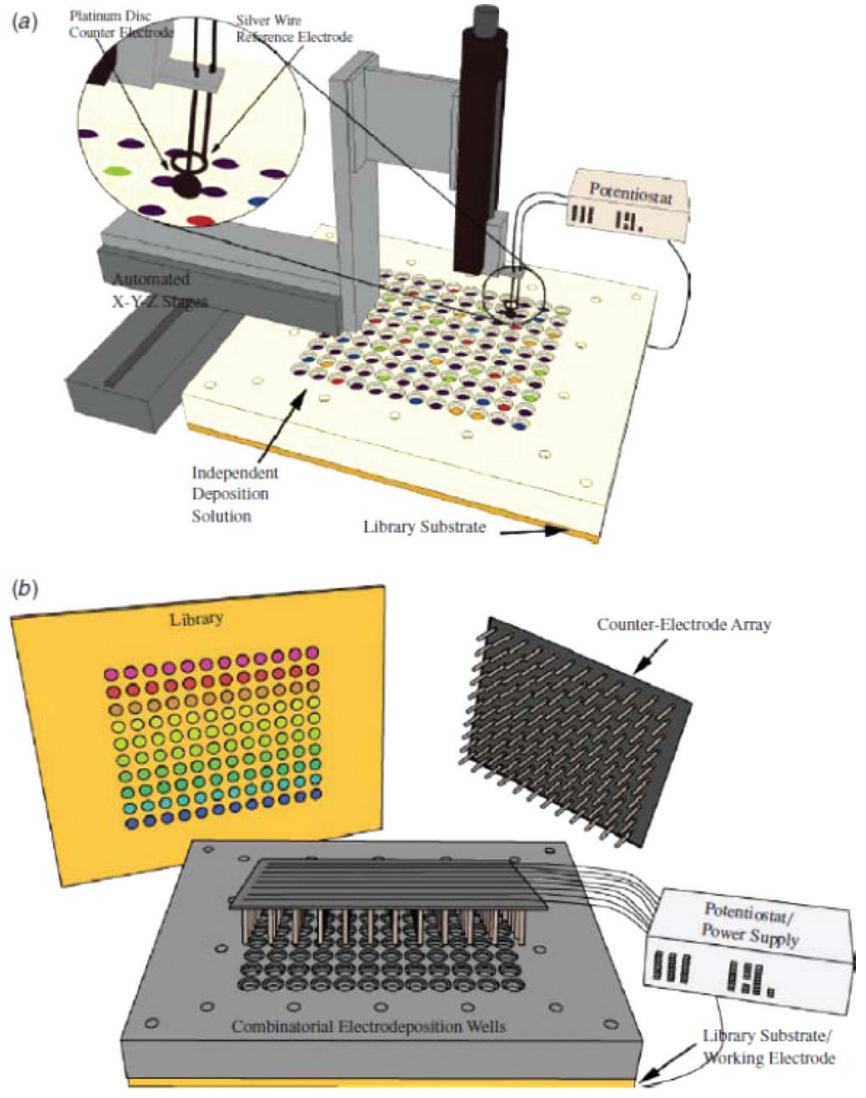

FIG. 76. Automated system for electrosynthesis. (a) Rapid serial deposition system; here, a moving arm is used to sequentially address each well, (b) parallel deposition system; here an array of counter electrodes are used to simultaneously electrodeposit each well. Reproduced with permission from B. Sung-Hyeon et al., Meas. Sci. Technol. 16, 54 (2005). Copyright 2005 Institute of Physics.

A more quantitative approach employs multielectrode fuel cell stacks to enable the direct measurement of $\mathrm{C}-\mathrm{V}$ characteristics of the catalyst. ${ }^{367-371}$ Statistical analysis of electrochemical experiments run on a 40 cell stack showed results that were consistent with single cell experiments. ${ }^{370}$ Further, in this research, the cell stack was optimized for catalyst loading and the concentration of methanol in the feed..$^{369,370}$ Another study used a well characterized array membrane assembly fuel cell, capable of characterizing up to twenty-five separate electrodes, in combination with a statistical experimental design to benchmark several potential synthesis techniques for Pt-Ru DMFC anode catalysts against commercially available catalysts, ${ }^{367,368,372,373}$ Fig. 77; the commercially available Pt-Ru catalysts prepared by Johnson Matthey outperforming the other synthesis techniques

Of all the single element catalysts, Pt shows the highest activity for methanol oxidation at temperatures compatible with fuel cells with polymer membranes. However, CO, which forms during the oxidation of methanol, strongly adsorbs to Pt surfaces, inhibiting usage of catalytically active sites. The combination of $\mathrm{Pt}$ with an oxophilic material, such as $\mathrm{Ru}$, can alleviate this problem. ${ }^{355}$ When $\mathrm{Ru}$ is substituted into Pt, it has a tendency to be leached from the surface of the catalyst during electrochemical cycling. To circumvent this issue, the ordered intermetallic phases $\mathrm{PtBi}$ and $\mathrm{PtPb}$ have recently come to attention. Using fluorescence imaging 


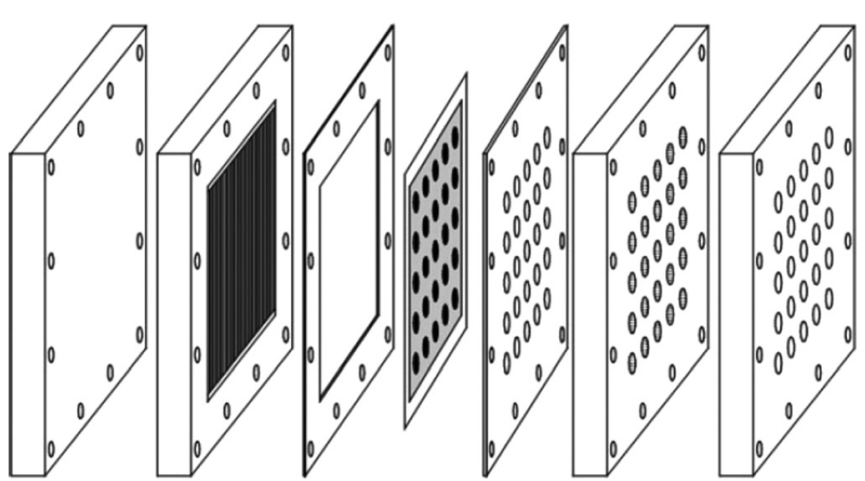

FIG. 77. Schematic of an array fuel cell with common counter electrode and array working electrode. Reproduced with permission from E. S. Smotkin and R. R. Diaz-Morales, Ann. Rev. Mater. Res. 33, 557 (2003). Copyright 2003 Annual Reviews.

and SEM to screen Pt-Bi-Pb ternary and Pt-Ti-Co-Pb quaternary combinatorial thin film libraries for their activity to methanol decomposition, ${ }^{349,360,374}$ it was found that by lightly doping a $\mathrm{PtPb}_{0.53}$ alloy with $\mathrm{Bi}$ the onset potential of methanol reduction could be decreased by $50 \mathrm{mV}$. XRD and XPS analyses showed the material to have a structure similar to $\mathrm{PtPb}$. Although the full oxidation of methanol produces only $\mathrm{CO}_{2}$ and $\mathrm{H}_{2}$, several other partial oxidation products can be generated, such as formic acid and formaldehyde. An ideal catalyst would have maximum selectivity towards full oxidation, but the characterization techniques discussed above can only determine overall activity. A scanning differential electrochemical mass spectrometer, which enables spatially resolved (on the order of the diameter of the capillary) investigations of the product phases during electrochemical catalysis, was devised. ${ }^{375}$ In this technique, the rastering electrode was a hollow capillary, which fed directly into a mass spectrometer, and a hydrophobic membrane was used to isolate the electrolyte from the high vacuum of the MS. High sensitivity measurements were demonstrated by detection of the oxidation of a monolayer of $\mathrm{CO}$ on a $\mathrm{Pt}$ surface. $^{351}$

The effects of physically mixing different compositions of Pt-Ru catalysts with various proportions of Nafion (a sulfonated tetrafluoroethylene based fluoropolymer with high protonic conductivity), through a multi-tiered screen were investigated. ${ }^{376}$ The best catalysts, as identified by cyclic voltammetry and impedance spectroscopy were evaluated in a DMFC application. It was found that a Pt:Ru ratio of $1: 1$ mixed with 36.4 wt. \% Nafion exhibited the highest activity and the lowest initial activation and $\mathrm{CO}$ desorption potentials; a synergistic effect between the Nafion and the active metal was proposed that involves Nafion providing protonic sites to promote $\mathrm{CO}$ oxidation.

Attempts to further increase current densities, lower the onset potential, and stabilize the anodes to the acidic environment of the DMFC have resulted in several investigations of ternary and quaternary additions to Pt-Ru. An array of 64 individually addressable electrodes was implemented to measure structure-composition-activity-stability maps of the Pt-Ru-Co ternary system. ${ }^{352}$ The results indicated that thin film catalysts with the nominal composition of $\mathrm{Pt}_{18} \mathrm{Co}_{62} \mathrm{Ru}_{20}$ exhibited a 10 to 30 times improvement in activity over pure $\mathrm{Pt}$, with negligible evidence of chemical attack. A thin film catalyst with similar composition $\left(\mathrm{Pt}_{15} \mathrm{Co}_{50} \mathrm{Ru}_{38}\right)$ was also reported. ${ }^{350}$ The proposed catalyst exhibited current densities 2.5 times those of conventional $\mathrm{Pt}_{90} \mathrm{Ru}_{10}$, in addition to a favorable onset potential of $0.22 \mathrm{~V}$, compared to a saturated calomel electrode. The discovery of a $\mathrm{Pt}_{44} \mathrm{Ru}_{12} \mathrm{~W}_{44}$ catalyst formulation $^{350}$ which exhibited current densities 1.5 times those of the best binary $\mathrm{Pt}-\mathrm{Ru}$ catalysts was also reported; however, the high $\mathrm{W}$ content in the alloy tended to make it unstable in an acidic environment. Further, a scanning electrochemical microscope was used to analyze activity of Pt-Ru-Mo catalysts for electro-oxidation of hydrogen and CO. ${ }^{377}$ Fluorescence imaging was used to determine the activity of $\mathrm{Pt}_{77} \mathrm{Ru}_{17} \mathrm{Mo}_{4} \mathrm{~W}_{2}$, a promising quaternary catalyst alloy for DMFC applications. This catalyst was then benchmarked against a standard $\mathrm{Pt}_{50} \mathrm{Ru}_{50}$ catalyst formulation, ${ }^{353}$ and showed roughly twice the current density after one hour of exposure to methanol. The Pt-Ru-Ni-Ti quaternary system was also investigated, with particular attention to mitigating the effects of surface morphology and segregation effects. ${ }^{378}$ A low Pt content catalyst $\left(\mathrm{Ni}_{31} \mathrm{Zr}_{13} \mathrm{Pt}_{33} \mathrm{Ru}_{23}\right)$ was discovered $^{378}$ which exhibited similar current densities to $\mathrm{Pt}_{84} \mathrm{Ru}_{16}$ catalysts, with an onset voltage that was $150 \mathrm{mV}$ lower, Fig. 78. XPS analysis revealed a substantially shifted Pt binding energy, possibly reflecting a change in the electron orbitals directly responsible for the catalytic behavior typically observed in Pt.

Arrays of different noble and TMs on a variety of supports ${ }^{379}$ were studied to investigate the effect of metal/oxide combinations on methanol decomposition and the water gas shift reaction (i.e., the reaction of $\mathrm{CO}$ and $\mathrm{H}_{2} \mathrm{O}$ to form $\mathrm{CO}_{2}$ and $\mathrm{H}_{2}$ ). It was found that $\mathrm{Pd} / \mathrm{MnO}_{2}$ performed the best for both reactions. Further ranking of the catalyst/support combinations revealed discrepancies between reactions, suggesting incomplete understanding of the underlying mechanism. A 64 element half-cell array was used to investigate the effect of Pt loading and particle size on carbon supports. ${ }^{380}$ It was found that smaller particle sizes resulted in fine structure in the voltammetry data that were not present in samples with larger particle sizes, most likely associated with an increase in edge sites on the small particles. Physical mixtures of $\mathrm{Au} / \mathrm{TiO}_{2}$, a high activity $\mathrm{CO}$ oxidation catalyst, with $\mathrm{PtRu} / \mathrm{C}$ were investigated to increase methanol reduction activity. ${ }^{381}$ A reduced oxidation potential as compared to pure $\mathrm{PtRu} / \mathrm{C}$ was observed for proportions of $\mathrm{PtRu}: \mathrm{Au} / \mathrm{TiO}_{2}$ of 9:1, 8:2, and 7:3. Further, electrochemically synthesized mixtures of $\mathrm{Pt}-\mathrm{WO}_{3}$ were studied in a combined synthesis and characterization high throughput system. ${ }^{382} \mathrm{Pt}-\mathrm{Zn}$ alloys were characterized, and it was found that $\mathrm{Zn}$ in $\mathrm{Zn}$-rich alloys leached until a PtZn phase with a presumed Pt rich skin was formed. ${ }^{383}$ There has only been sparse work devoted to the cathode (oxygen reducing) side of the polymer fuel cell membrane. ${ }^{384}$ One study was devoted to screening the Pt-Ni-Zr ternary system for oxygen reduction reaction properties. ${ }^{385}$ In a related work, 5 Pt group metals $(\mathrm{Pt}, \mathrm{Ru}, \mathrm{Os}, \mathrm{Ir}$, and $\mathrm{Rh}$ ) systems were screened for their bifunctional oxygen reduction and water oxidation properties; it was demonstrated that replacing most of the Ir in PtIr 

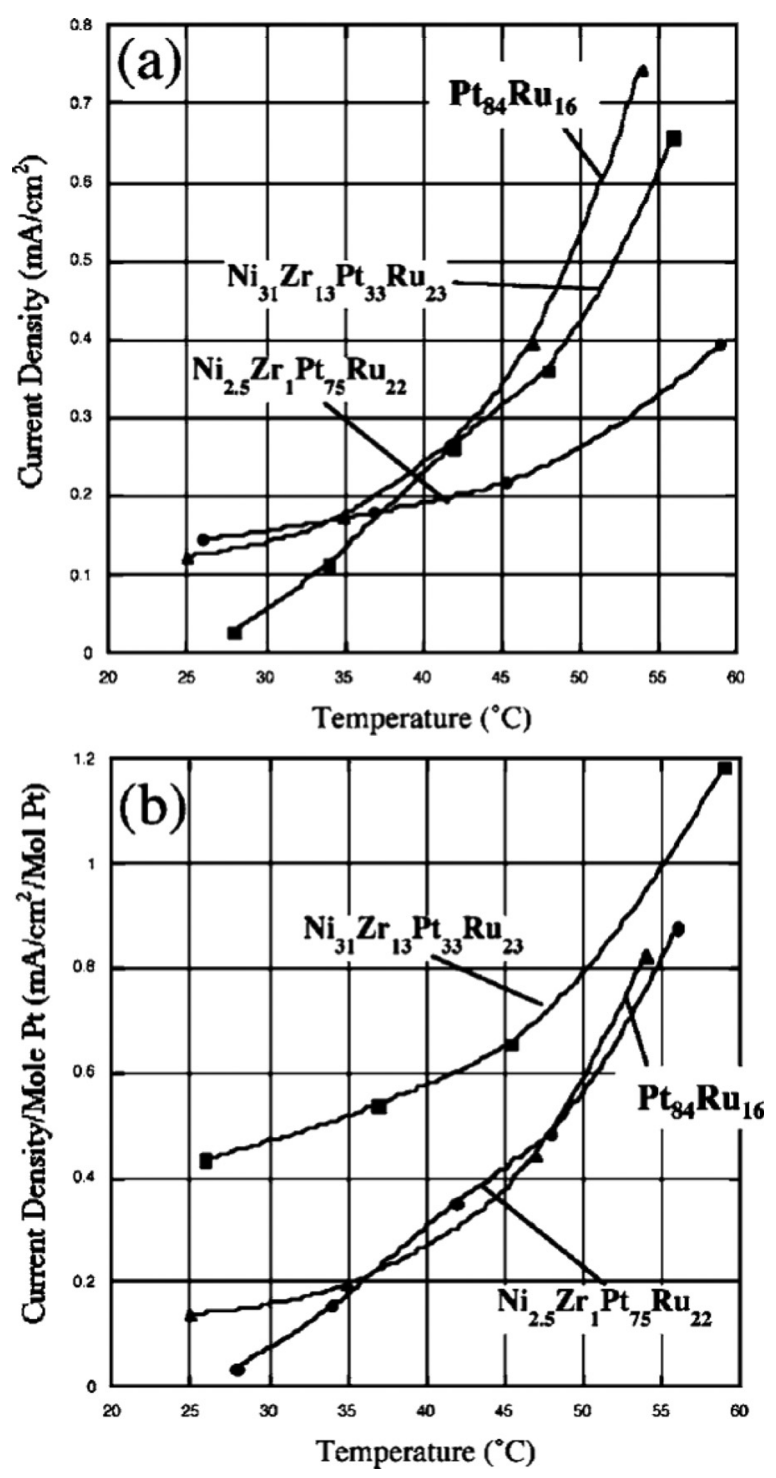

FIG. 78. (a) Plot comparing the current density of a Ni-Zr-Pt-Ru catalyst as a function of temperature for a catalyst composition discovered by Whitacre. (b) Same data normalized to mole fraction Pt per unit area. Reproduced with permission from J. F. Whitacre et al., J. Electrochem. Soc. 152, A1780 (2005). Copyright 2005 The Electrochemical Society, Inc.

alloys with $\mathrm{Ru}$ improved catalyst activity. ${ }^{386} \mathrm{~A}$ coupled theoretical-experimental approach was recently demonstrated by Lee et al. in discovering novel binary oxygen reduction reaction catalysts. ${ }^{387}$ By calculating the oxygen adsorption energy for a series of compounds and comparing them to the value for $\mathrm{Pt}$, a new $\mathrm{Pd}-\mathrm{Co}$ alloy was predicted and subsequently experimentally verified.

Finally, ceria $\left(\mathrm{Ce}_{2} \mathrm{O}_{3}\right)$ based mixed oxide conductors have been under extensive investigation over the past $10 \mathrm{yr}$ as potential combined catalysts/anodes for SOFC's. ${ }^{388}$ The goal is to replace $\mathrm{Ni} / \mathrm{ZrO}_{2}$ anodes, which suffer from coke formation during hydrocarbon reforming, which plugs the SOFC and causes power loss. Metal doping of $\mathrm{Ce}_{2} \mathrm{O}_{3}$ based catalysts and its effect on methane reforming has been studied. ${ }^{356,389}$ Ink jet printing of oxide/carbonate suspensions was used to create libraries in the La-Sr-Fe-Mn-O system, with particular emphasis on identifying new conducting perovskite phases. ${ }^{390}$ Subsequent ionic conduction measurements were attempted, but significant contamination of the perovskite surfaces prevented definitive correlations between composition and ionic conductivity from being established. ${ }^{357,391}$ Although it is well-known that several companies developed highthroughput techniques to screen potential fuel cell materials, there have been few reports in the literature regarding their efforts. One published example involved the development of a high-throughput method for synthesizing, evaluating, and characterizing new low temperature SOFC materials. ${ }^{392}$

\section{HIGH-THROUGHPUT MAPPING OF PHASE DIAGRAMS}

As discussed in Sec. IV C, mapping of compositional phase diagrams is an integral part of any materials exploration effort. Not surprisingly, combining information regarding distribution of structural phases across phase diagrams with mapping of functional properties represents a particularly powerful way to rapidly identify new compositions and compounds with enhanced physical properties. In the fields of metallurgy and ceramics, mapping binary and higherorder phase diagrams through bulk compound synthesis is a traditional and critical task. However, despite concerted global efforts by materials scientists, a large fraction of all possible ternary and multinary alloys remain experimentally unexplored. In fact, the history of materials science is paved with efforts to understand and map phase diagrams. The high-throughput thin film library approach, combined with scanning XRD experiments represents a tremendous opportunity, as had been recognized as long as about 50 years ago. $^{12,393}$ Bulk high-throughput efforts such as diffusion multiples ${ }^{136}$ and directed laser deposition ${ }^{394}$ have also been successfully implemented to map structural phases in composition gradient samples.

There have been many demonstrations of rapid mapping of structural phases using synchrotron-based XRD. Chu et al $^{395}$ studied epitaxially grown CCS library films in the Co-Mn-Ge system. It has also been used to map structural phases across a Ni-Fe-Cr composition spread; ${ }^{396}$ comparison of the measured phase distribution from a CCS library sample annealed at $800^{\circ} \mathrm{C}$ corresponds well to the equilibrium diagram calculated for the same temperature, as shown in Fig. 79. The synchrotron XRD mapping exercise is particularly useful when combined with x-ray fluorescence for composition determination. ${ }^{180,397,398}$ The same combination of techniques (although not using a synchrotron) has been effectively used to map composition regions of metallic glasses and amorphous metal systems. ${ }^{399}$

Given the speed with which structural data can be acquired in, for example, the aforementioned synchrotron experiments, the rapid analysis of large amounts of raw XRD data becomes a challenge. There have been a number of efforts directed at developing algorithms that allow rapid identification of key trends and features in diffraction data taken across multiphase CCS libraries, and subsequent attribution of such data to individual phases. ${ }^{400-407}$ By performing cluster analysis, similar diffraction patterns, even among hundreds of spectra, can be rapidly identified. Similar XRD patterns usually indicate the presence of similar phases in 


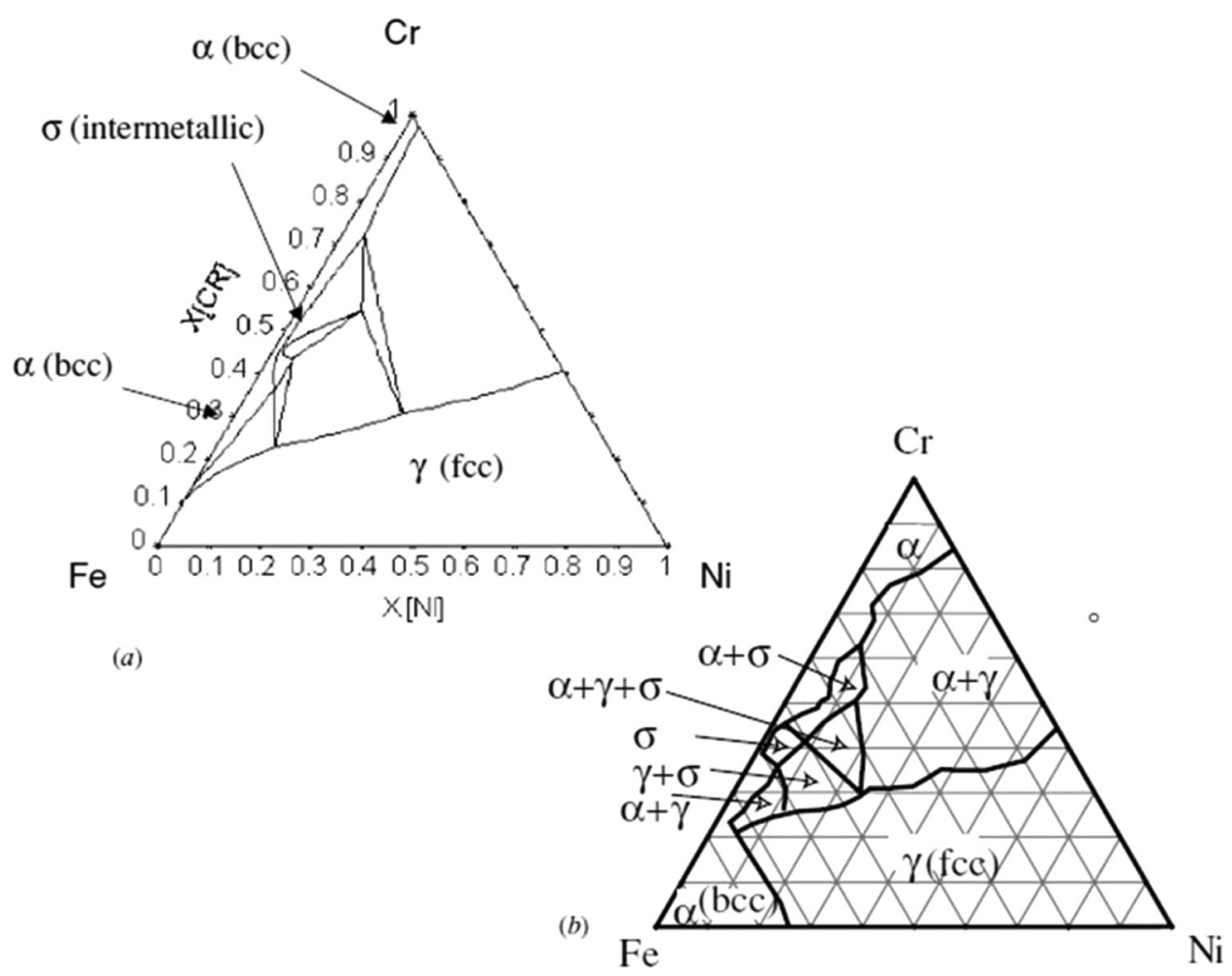

FIG. 79. (a) Calculated ternary diagram of $\mathrm{Ni}-\mathrm{Fe}-\mathrm{Cr}$ system at $875^{\circ} \mathrm{C}$ and (b) measured ternary diagram from a CCS library film. Reproduced with permission from A. Rar et al., Meas. Sci. Technol. 16, 46 (2005). Copyright 2005 Institute of Physics.

a particular composition region. Thus, one goal of these algorithms is to automatically identify mixtures of crystallographic phases, even when significant diffraction peak shifting, for example due solid solution substitutional effects, is present. These analysis techniques are extremely useful in directly and visually delineating the composition-structureproperty relationships of materials systems. Many of the algorithms developed for handling large amount of XRD data are also applicable to rapid analysis of other spectra such as Raman, XPS, and FTIR data. ${ }^{408}$

As a general comment, computational combinatorial approaches have recently received much attention with the arrival of the Materials Genome Initiative (MGI). ${ }^{409}$ To take advantage of the power of high-throughput methodologies, an integrated strategy combining computational methods with rapid experimental verification work is required. Thus, going forward, the challenge for combinatorial methodology is the effective coupling of synthesis, characterization, and theory. ${ }^{410-414}$ This will almost certainly require an entirely new type of flexible management system for "big data," which can rapidly handle large amount of data in a variety of formats.

Accurate structural phase mapping can directly facilitate rapid discovery of functional materials, whose interesting properties are derived from phase transformations. Morphotropic phase boundary piezoelectrics, discussed in Sec. III, are a good example. Further, SMAs undergo structural transitions as a result of martensitic phase transformations, going from high temperature, higher symmetry parent phases to low temperature, lower symmetry martensites. The technological applications of many SMAs depends on operating the alloys in the temperature range of their phase transformations; for example, superelasticity, a property that derives from stress-induced transformations, can be used to accommodate large stains in mechanical framework for a variety of applications including dental braces, peripheral vascular stents, and eyeglass frames. ${ }^{415}$ In one study, a combinatorial investigation was carried out to identify those SMA compositions with minimal martensitic transformation thermal hysteresis. ${ }^{416}$ It is known that minimizing the hysteresis would increase the fatigue life of SMAs, and thus, significantly improve the long-term performance of many SMA applications. Guided by the non-linear theory of martensite developed by Ball and James, ${ }^{417}$ combinatorial mapping of the lattice constants of Ni-Ti-Cu SMAs was

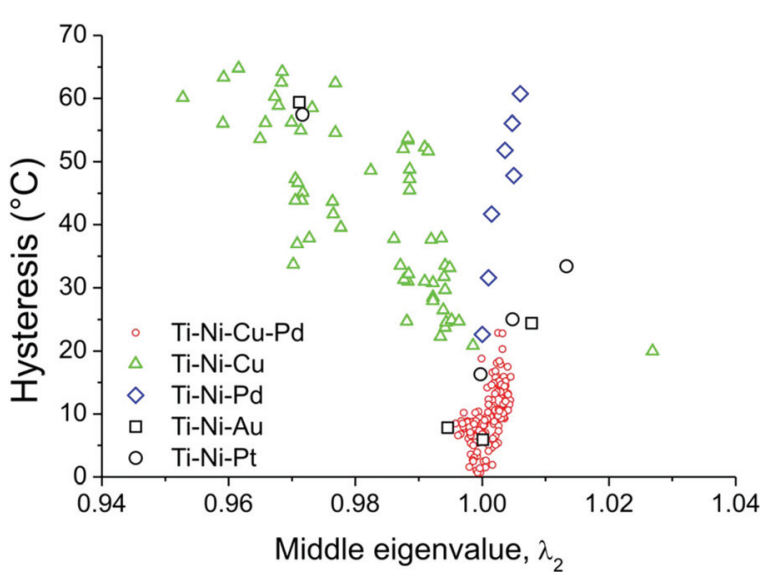

FIG. 80. Thermal hysteresis (measured by resistive transitions) vs. middle eigenvalues (determined from lattice constants) for different Ni-Ti-X-Y systems shows that making the middle eigenvalue go to 1 (by satisfying lattice constant conditions set by the non-linear theory of martensite) reduces the thermal hysteresis of martensitic transformation. Each data point corresponds to a different composition. Reproduced with permission from R. Zarnetta et al., Adv. Funct. Mater. 20, 1917 (2010). Copyright 2010 Wiley_VCH Verlag Gmbh \& Co. 

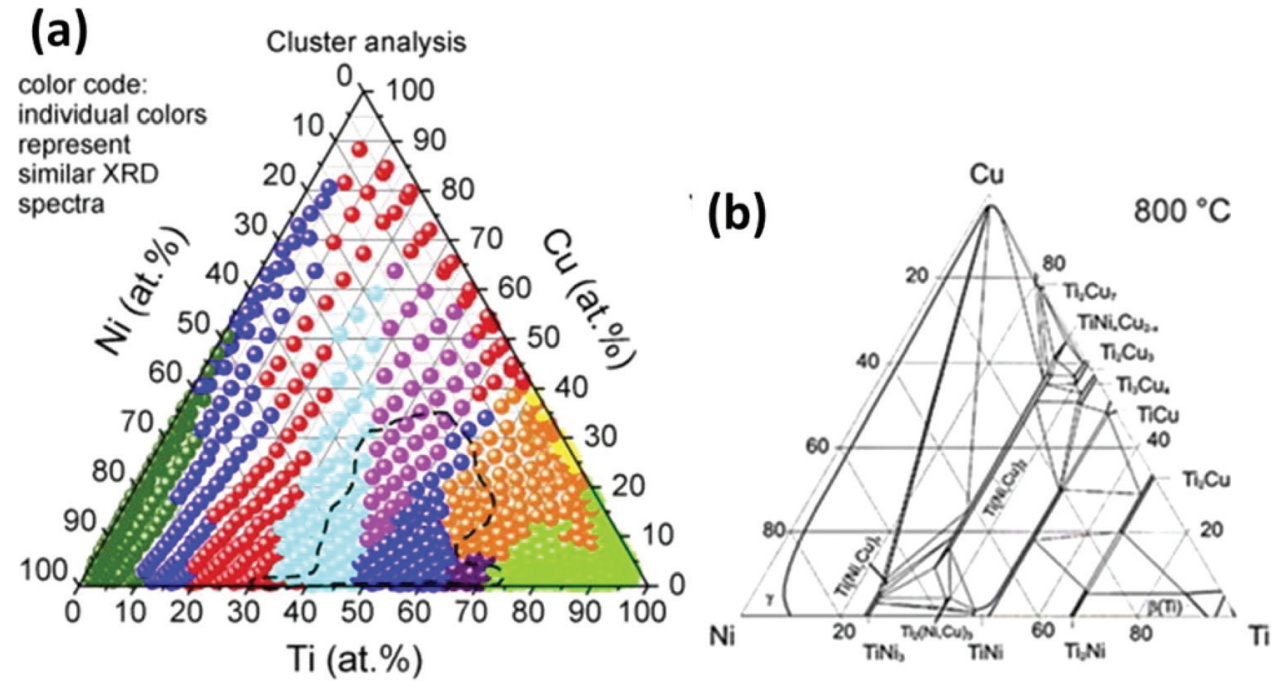

FIG. 81. (a) Rapid cluster analysis of diffraction spectra taken from a Ni-Ti-Cu composition spread. Different colors indicate spectra with similar patterns reflecting the different structural regions. Reproduced with permission from R. Zarnetta et al., Intermetallics 26, 98 (2012). Copyright 2012 Elsevier BV. (b) High-temperature phase diagram of Ni-Ti-Cu obtained using bulk samples. The clear resemblance between (a) and (b) attest to the validity of the composition spread and the cluster analysis technique for quick delineation of structural phase distributions across large compositional ranges (see Ref. 438). Reproduced with permission from F. J. J. Vanloo et al., J. Less-Common Met. 57, 111 (1978). Copyright 1978 Elsevier Publishing Co.

carried out through synchrotron XRD. This study revealed that satisfying the lattice constant conditions set forth by the theory indeed leads to SMAs with minimal hysteresis. In a follow-on study, the quaternary system Ni-Ti-Cu-Pd was investigated to further improve the composition, and focus on "zero-hysteresis" compounds, as determined by thin film measurements. ${ }^{418}$ Figure 80 summarizes this research; it can be seen that minimization of the thermal hysteresis was achieved through tailoring the lattice constants of the alloys. As part

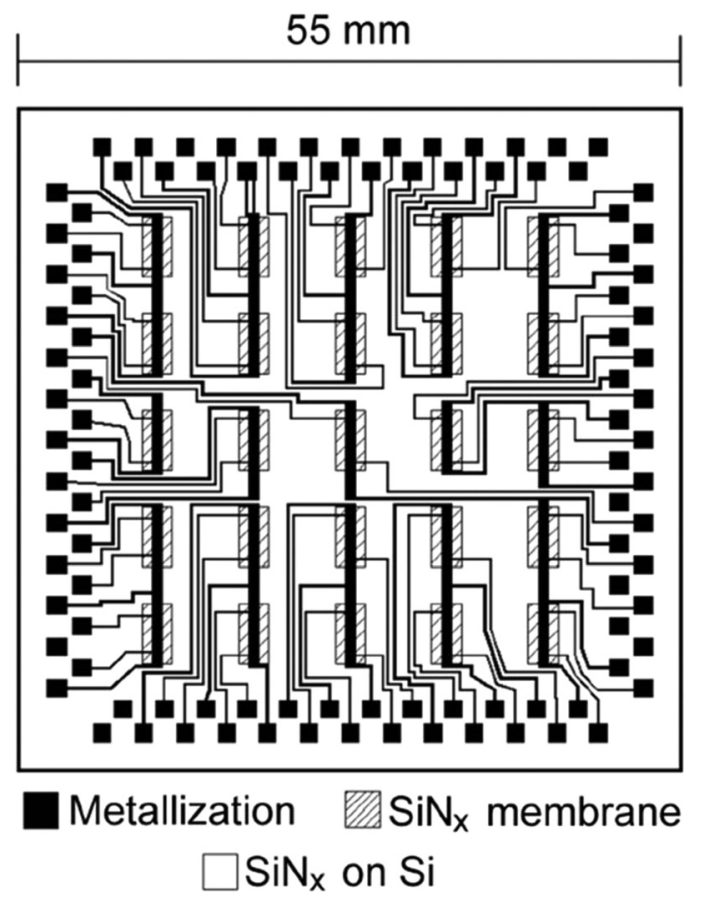

FIG. 82. Schematic of the parallel nano-scanning calorimeter (see Ref. 439). Reproduced with permission from P. J. McCluskey et al., Acta Mater. 59, 5116 (2011). Copyright 2011 Pergamon. of the same study, it was shown that the "zero-hysteresis" compositions identified from the CCS experiments, when processed as bulk samples, indeed showed substantially enhanced functional stability due to minimized fatigue behavior. Zarnetta et al. ${ }^{419,420}$ have also investigated the details of phase transformation behavior across a variety of related SMA systems. Figure 81 shows one such study where data cluster analysis was performed on XRD spectra from a Ni-Ti-Cu CCS library. The clear resemblance to the known bulk high-temperature phase diagram (Fig. 81(b)) speaks to the effectiveness of the combinatorial approach. ${ }^{421}$

Other methods of mapping phase diagrams of SMAs involve their mechanical properties. Since in their martensitic state, they are mechanically soft, nanoindentation mapping of elastic modulus has been effectively used to identify martensite composition regions. ${ }^{422}$ Another method for detection of the martensitic transition is through implementation of arrays of cantilevers, since the change in the elastic modulus of the film deposited atop the cantilever as a result of the martensitic transition results in an abrupt deflection. ${ }^{108}$ Indeed, cantilever arrays have served as a versatile combinatorial platform for mapping a variety of mechanical properties such as film stresses, glass transitions, and fatigue. ${ }^{311,423-426}$ Finally, other dense MEMS sensor arrays, such as microhotplates for nanocalorimetry and gas sensors, have been very useful in high throughput measurements. ${ }^{427-429}$ Figure 82 illustrates a nanocalorimeter array device.

Another class of materials that has advanced as a result of high throughput mapping of properties are ternary compounds with a composition of $M_{n+1} A X_{n}$ ("MAX" phases) where $\mathrm{n}=1,2$, or $3, \mathrm{M}$ is a TM, A is a III-A or IV-A Group element, and $\mathrm{X}$ is carbon or nitrogen. Currently, more than 50 distinct MAX phases have been synthesized in bulk. ${ }^{430}$ There is much interest in this class of refractory materials due to their machinability and extreme hardness. ${ }^{431-433}$ Further, since they exhibit low friction and oxidation resistance, ${ }^{430}$ 
these materials are good candidates for protective coatings. However, much remains unknown about MAX phases, especially with regard to the properties of solid solutions. In recent research, nanoindentation mapping was carried out on CCS films of $\left(\mathrm{Ti}_{1-\mathrm{x}} \mathrm{Nb}_{\mathrm{x}}\right)_{2} \mathrm{AlC}$ and $\left(\mathrm{Cr}_{1-\mathrm{x}} \mathrm{V}_{\mathrm{x}}\right)_{2} \mathrm{GeC}$, and it was discovered that some solid solutions have larger elastic modulus compared to their end members. ${ }^{434,435}$

\section{ACKNOWLEDGMENTS}

One of the authors (IT) is supported by the following grants: NSF-MRSEC (DMR 0520471), ARO W911NF-07-10410, AFOSR MURI FA95500910603, and Beyond Rare-Earth Permanent Magnets (DOE EERE).

\section{NOMENCLATURE}

$\mathrm{ALD}=$ atomic layer deposition

$\mathrm{APCVD}=$ atmospheric pressure chemical vapor deposition

$\mathrm{BH}_{\max }=$ maximum energy product

$\mathrm{CCD}=$ charge coupled display

$\mathrm{CCS}=$ continuous composition spread

$\mathrm{CMOS}=$ complementary metal oxide semiconductor

$\mathrm{CRT}=$ cathode ray tube

$\mathrm{C}-\mathrm{V}=$ capacitance-voltage

$\mathrm{CVD}=$ chemical vapor deposition

$\mathrm{DFT}=$ density function theory

$\mathrm{DMFC}=$ direct methanol fuel cell

$\mathrm{EPMA}=$ electron probe microanalysis

FTIR $=$ Fourier transform infrared spectroscopy

FYNES = fluorescence yield near edge spectroscopy

ITO $=$ indium tin oxide

ITRS $=$ International Technology Roadmap for Semiconductors

$\mathrm{I}-\mathrm{V}=$ current-voltage

$\mathrm{IR}=$ infrared

MEMS $=$ microelectromechanical systems

$\mathrm{MFM}=$ magnetic force microscopy

MOCVD = metal-organic chemical vapor deposition

MOKE $=$ magnetic-optical Kerr effect

$\mathrm{MPB}=$ morphotropic phase boundary

$\mathrm{NIR}=$ near infrared

$\mathrm{NMOS}=\mathrm{n}$-channel metal oxide semiconductor

$\mathrm{PCM}=$ phase change material

$\mathrm{PEM}=$ polymer electrolyte membrane

$\mathrm{PFM}=$ piezoresponse force microscopy

PGAA $=$ prompt gamma activation analysis

PLD $=$ pulsed laser deposition

PMOS $=$ p-channel metal oxide semiconductor

$\mathrm{PV}=$ photovoltaic

$\mathrm{PZT}=$ Lead zirconium titanate

RBS $=$ Rutherford backscattering

$\mathrm{RE}=$ rare earth

$\mathrm{RF}=$ radio frequency

RHEED $=$ reflection high energy electron diffraction

$\mathrm{RT}=$ Room temperature

SEM = scanning electron microscopy

SEMPA = scanning electron microscopy polarization analysis
$\mathrm{SMA}=$ shape memory alloy

$\mathrm{SMM}=$ scanning microwave microscopy

$\mathrm{SOFC}=$ solid oxide fuel cell

SQUID $=$ superconducting quantum interference device

SSM = scanning SQUID microscopy

$\mathrm{STO}=$ strontium titanate

$\mathrm{TCO}=$ transparent conducting oxide

TDTR $=$ time domain thermoreflectance

$\mathrm{TEM}=$ transmission electron microscopy

$\mathrm{TIO}=$ tin indium oxide

$\mathrm{TM}=$ transition metal

$\mathrm{UV}=$ ultraviolet light

$\mathrm{VASP}=$ Vienna $A b$ Initio Simulation Program

$\mathrm{VIS}=$ visible light

WDS = wavelength dispersive spectroscopy

$\mathrm{XMCD}=\mathrm{X}$-ray magnetic circular dichroism

$\mathrm{XPS}=\mathrm{x}$-ray photoemission spectroscopy

$\mathrm{XRD}=\mathrm{X}$-ray diffraction

$\mathrm{ZITO}=$ zinc indium tin oxide

${ }^{1}$ N. K. Terrett, M. Gardner, D. W. Gordon, R. J. Kobylecki, and J. Steele, Tetrahedron 51(30), 8135-8173 (1995).

${ }^{2}$ P. P. Pescarmona, J. C. van der Waal, I. E. Maxwell, and T. Maschmeyer, Catal. Lett. 63(1-2), 1-11 (1999).

${ }^{3}$ L. A. Thompson and J. A. Ellman, Chem. Rev. 96(1), 555-600 (1996).

${ }^{4}$ R. B. Merrifield and J. M. Stewart, Nature 207(4996), 522 (1965).

${ }^{5}$ B. J. Chisholm and D. C. Webster, J. Coat. Technol. Res. 4(1), 1-12 (2007).

${ }^{6}$ R. A. Potyrailo and V. M. Mirsky, Chem. Rev. 108(2), 770-813 (2008).

${ }^{7}$ H. Koinuma and I. Takeuchi, Nature Mater. 3, 429-438 (2004).

${ }^{8}$ X. D. Xiang, X. D. Sun, G. Briceno, Y. L. Lou, K. A. Wang, H. Y. Chang, W. G. Wallace-Freedman, S. W. Chen, and P. G. Schultz, Science 268(5218), 1738-1740 (1995).

${ }^{9}$ X. D. Xiang and P. G. Schultz, Physica C 282, 428-430 (1997).

${ }^{10}$ I. Takeuchi, R. B. van Dover and H. Koinuma, MRS Bull. 27(4), 301-308 (2002).

${ }^{11}$ A. Boettcher, G. Haase, and R. Thun, Z. Metallkd. 46(5), 386-400 (1955).

${ }^{12}$ K. Kennedy, T. Stefansky, G. Davy, V. F. Zackay, and E. R. Parker, J. Appl. Phys. 36(12), 3808 (1965).

${ }^{13}$ J. J. Hanak, J. Mater. Sci. 5(11), 964 (1970).

${ }^{14}$ A. Boettcher and R. Thun, Z. Anorg. Allg. Chem. 283(1-6), 26-48 (1956).

${ }^{15}$ R. W. Cahn, The Coming of Materials Science (Pergamon Press, Oxford, 2001).

${ }^{16}$ D. D. L. Chung, Functional Materials (World Scientific Publishing Company, Singapore, 2010).

${ }^{17}$ N. Boehmer, T. Roussiere, M. Kuba, and S. A. Schunk, Comb. Chem. High Throughput Screening 15(2), 123-135 (2012).

${ }^{18}$ S. C. Fung and T. C. Ho, USA Patent No. 7390664 (2008).

${ }^{19}$ X. D. Xiang, H. Yang, G. Wang, and J. Melman, USA Patent No. 7704923 (2010).

${ }^{20}$ C. G. Lugmair, A. Hagemayer, L. Van Erden, A. F. Volpe, D. M. Lowe, and Y. Liu, USA Patent No. 7503515 (2009).

${ }^{21}$ B. E. Hayden, C. E. Lee, C. Mormiche, and D. Thompsett, USA Patent No. 7901835 (2011).

${ }^{22}$ J. C. Dellamorte, M. A. Barteau, and J. Lauterbach, Surf. Sci. 603(10-12), 1770-1775 (2009).

${ }^{23}$ G. Kirsten and W. F. Maier, Appl. Surf. Sci. 223(1-3), 87-101 (2004).

${ }^{24}$ W. F. Maier, K. Stoewe, and S. Sieg, Angew. Chem., Int. Ed. 46(32), 6016-6067 (2007).

${ }^{25}$ J. M. Newsam and F. Schuth, Biotechnol. Bioeng. 61(4), 203-216 (1999).

${ }^{26}$ D. C. Webster, Macromol. Chem. Phys. 209(3), 237-246 (2008).

${ }^{27}$ S. I. Woo, K. W. Kim, H. Y. Cho, K. S. Oh, M. K. Jeon, N. H. Tarte, T. S. Kim, and A. Mahmood, QSAR Comb. Sci. 24(1), 138-154 (2005).

${ }^{28}$ C. G. Simon and S. Lin-Gibson, Adv. Mater. 23(3), 369-387 (2011).

${ }^{29}$ J. Genzer, Soft Matter Gradient Surfaces: Methods and Applications (John Wiley and Sons, 2012). 
${ }^{30} \mathrm{X}$. D. Xiang and I. Takeuchi, Combinatorial Materials Synthesis (Marcel Dekker, Inc., New York, 2003).

${ }^{31}$ Z. H. Barber and M. G. Blamire, Mater. Sci. Technol. 24(7), 757-770 (2008).

${ }^{32}$ J. N. Cawse, Experimental Design for Combinatorial and High Throughput Materials Development (John Wiley and Sons, New York, 2003).

${ }^{33}$ R. A. Potyrailo and E. J. Amis, High throughput analysis : a tool for combinatorial materials science (Kluwer Academic/Plenum Publishers, New York, 2003).

${ }^{34}$ See http://www.itrs.net/Links/2011ITRS/Home2011.htm for the International Technology Roadmap for Semiconductors (ITRS), which discusses future materials needs for the microelectronics industry (as well as many other needs) (2011).

${ }^{35}$ Z. Fresco, A. Karamcheti, N. Kalyankar, P. Zhang, and D. Srinivas, Solid State Technol. 50(10), 39-43 (2007).

${ }^{36}$ M. L. Green, E. P. Gusev, R. Degraeve, and E. L. Garfunkel, J. Appl. Phys. 90(5), 2057-2121 (2001).

${ }^{37}$ D. A. Muller, T. Sorsch, S. Moccio, F. H. Baumann, K. Evans-Lutterodt, and G. Timp, Nature 399(6738), 758-761 (1999).

${ }^{38}$ G. D. Wilk, R. M. Wallace, and J. M. Anthony, J. Appl. Phys. 89(10), 5243-5275 (2001)

${ }^{39}$ R. B. van Dover, L. D. Schneemeyer, and R. M. Fleming, Nature 392(6672), 162-164 (1998).

${ }^{40}$ T. Chikyow, K. Hasegawa, T. Tamori, K. Ohmori, N. Umezawa, K. Nakajima, K. Yamada, and H. Koinuma, in 2006 8th International Conference on Solid-State and Integrated Circuit Technology (IEEE Cat. No. 06EX1294) CD-ROM (2006), p. 6.

${ }^{41}$ K. Hasegawa, P. Ahmet, N. Okazaki, T. Hasegawa, K. Fujimoto, M. Watanabe, T. Chikyow, and H. Koinuma, Appl. Surf. Sci. 223(1-3), 229-232 (2004).

${ }^{42}$ T. Chikyow, K. Ohmori, T. Nagata, N. Umezawa, M. Haemori, M. Yoshitake, T. Hasegawa, H. Koinuma, K. Yamada, and IEEE, in 2008 International Symposium on Vlsi Technology, Systems and Applications (Vlsi-Tsa), Proceedings of Technical Program (2008), pp. 66-67.

${ }^{43}$ N. Okazaki, P. Ahmet, T. Chikyow, H. Odagawa, Y. Cho, T. Fukumura, M. Kawasaki, M. Ohtani, H. Koinuma, and T. Hasegawa, in Combinatorial and Artificial Intelligence Methods in Materials Science Symposium (Materials Research Society Symposium Proceedings) (2002), Vol. 700, p. 119.

${ }^{44}$ N. Okazaki, S. Okazaki, H. Higuma, S. Miyashita, Y. Cho, J. Nishimura, T. Fukumura, M. Kawasaki, M. Murakami, Y. Yamamoto, Y. Matsumoto, H. Koinuma, and T. Hasegawa, Appl. Surf. Sci. 223(1-3), 196-199 (2004).

${ }^{45}$ M.-Y. Ho, H. Gong, G. D. Wilk, B. W. Busch, M. L. Green, P. M. Voyles, D. A. Muller, M. Bude, W. H. Lin, A. See, M. E. Loomans, S. K. Lahiri, and P. I. Raisanen, J. Appl. Phys. 93, 1477 (2003).

${ }^{46}$ M. Y. Ho, H. Gong, G. D. Wilk, B. W. Busch, M. L. Green, W. H. Lin, A. See, S. K. Lahiri, M. E. Loomans, P. I. Raisanen, and T. Gustafsson, Appl. Phys. Lett. 81(22), 4218-4220 (2002).

${ }^{47}$ D. Kukuruznyak, H. Reichert, K. Ohmori, P. Ahmet, and T. Chikyow, Adv. Mater. 20(20), 3827-3831 (2008).

${ }^{48}$ D. A. Kukuruznyak, H. Reichert, J. Okasinski, H. Dosch, T. Chikyow, J. Daniels, and V. Honkimaki, Appl. Phys. Lett. 91, 071916 (2007).

${ }^{49}$ K. S. Chang, M. L. Green, J. R. Hattrick-Simpers, I. Takeuchi, J. S. Suehle, O. Celik, and S. De Gendt, IEEE Trans. Electron Devices 55(10), 2641-2647 (2008)

${ }^{50}$ N. D. Bassim, P. K. Schenck, M. Otani, and H. Oguchi, Rev. Sci. Instrum. 78(7), 072203 (2007).

${ }^{51}$ P. K. Schenck, N. D. Bassim, M. Otani, H. Oguchi, and M. L. Green, Appl. Surf. Sci. 254(3), 781-784 (2007).

${ }^{52}$ J. L. Klamo, P. K. Schenck, P. G. Burke, K. S. Chang, and M. L. Green, J. Appl. Phys. 107(5), 054101 (2010).

${ }^{53}$ K. J. Hubbard and D. G. Schlom, J. Mater. Res. 11(11), 2757-2776 (1996).

${ }^{54}$ K. S. Chang, M. L. Green, P. K. Schenck, I. Levin, and E. Venkatasubramanian, IEEE Trans. Electron Devices 59(12), 3212-3216 (2012).

${ }^{55}$ S. Toyoda, J. Okabayashi, M. Komatsu, M. Oshima, D. I. Lee, S. Y. Sun, Y. Sun, P. A. Pianetta, D. Kukuruznyak, and T. Chikyow, J. Vac. Sci. Technol. A 28(1), 16-19 (2010).

${ }^{56}$ J. O. Choo, R. A. Adomaitis, L. Henn-Lecordier, Y. Cai, and G. W. Rubloff, Rev. Sci. Instrum. 76(6), 062217 (2005).

${ }^{57}$ G. Hyett, M. A. Green, and I. P. Parkin, J. Am. Chem. Soc. 129(50), 15541-15548 (2007).
${ }^{58}$ B. Xia, F. Chen, S. A. Campbell, J. T. Roberts, and W. L. Gladfelter, Chem. Vap. Deposition 10(4), 195-200 (2004).

${ }^{59}$ M. Ritala and M. Leskela, in Handbook of Thin Film Materials: Deposition and Processing of Thin Films, edited by H. S. Nalwa (Academic Press, San Diego, 2002), Vol. 1.

${ }^{60}$ J. O. Choo, R. A. Adomaitis, G. W. Rubloff, L. Henn-Lecordier, and Y. J. Liu, AIChE J. 51(2), 572-584 (2005).

${ }^{61}$ Y. Cai, L. Henn-Lecordier, G. W. Rubloff, R. Sreenivasan, J.-O. Choo, and R. A. Adomaitis, J. Vac. Sci. Technol. B 25(4), 1288-1297 (2007).

${ }^{62}$ K. S. Chang, M. L. Green, J. Suehle, E. M. Vogel, H. Xiong, J. HattrickSimpers, I. Takeuchi, O. Famodu, K. Ohmori, P. Ahmet, T. Chikyow, P. Majhi, B. H. Lee, and M. Gardner, Appl. Phys. Lett. 89(14), 142108 (2006).

${ }^{63}$ P. Ahmet, Y. Z. Yoo, K. Hasegawa, H. Koinuma, and T. Chikyow, Appl. Phys. A: Mater. Sci. Process. 79(4-6), 837-839 (2004).

${ }^{64}$ P. Ahmet, T. Nagata, D. Kukuruznyak, S. Yagyu, Y. Wakayama, M. Yoshitake, and T. Chikyow, Appl. Surf. Sci. 252(7), 2472-2476 (2006).

${ }^{65}$ M. L. Green, K. S. Chang, S. DeGendt, T. Schram, and J. HattrickSimpers, Microelectron. Eng. 84(9-10), 2209-2212 (2007).

${ }^{66} \mathrm{~K}$. Ohmori, T. Chikyow, T. Hosoi, H. Watanabe, K. Nakajima, T. Adachi, A. Ishikawa, Y. Sugita, Y. Nara, Y. Ohji, K. Shiraishi, K. Yamabe, and K. Yamada, 2007 IEEE International Electron Devices Meeting - IEDM 2007, 345-348.

${ }^{67}$ K. Ohmori, P. Ahmet, K. Shiraishi, K. Yamabe, H. Watanabe, Y. Akasaka, N. Umezawa, K. Nakajima, M. Yoshitake, T. Nakayama, K. S. Chang, K. Kakushima, Y. Nara, M. L. Green, H. Iwai, K. Yamada, and T. Chikyow, in 2006 8th International Conference on Solid-State and Integrated Circuit Technology (IEEE Cat. No. O6EX1294) CD-ROM (2006), p. 4.

${ }^{68}$ W. P. Bai, S. H. Bae, H. C. Wen, S. Mathew, L. K. Bera, N. Balasubramanian, N. Yamada, M. F. Li, and D. L. Kwong, IEEE Electron Device Lett. 26(4), 231-233 (2005).

${ }^{69}$ K. Choi, H. N. Alshareef, H. C. Wen, H. Harris, H. Luan, Y. Senzaki, P. Lysaght, P. Majhi, and B. H. Lee, Appl. Phys. Lett. 89(3), 032113 (2006).

${ }^{70}$ C. S. Park, B. J. Cho, and D. L. Kwong, IEEE Electron Device Lett. 24(5), 298-300 (2003).

${ }^{71}$ Y. S. Suh, G. P. Heuss, J. H. Lee, and V. Misra, IEEE Electron Device Lett. 24(7), 439-441 (2003).

${ }^{72}$ H. N. Alshareef, K. Choi, H. C. Wen, H. Luan, H. Harris, Y. Senzaki, P. Majhi, B. H. Lee, R. Jammy, S. Aguirre-Tostado, B. E. Gnade, and R. M. Wallace, Appl. Phys. Lett. 88(7), 072108 (2006).

${ }^{73}$ K. S. Chang, M. L. Green, I. Levin, J. R. Hattrick-Simpers, C. Jaye, D. A. Fischer, I. Takeuchi, and S. De Gendt, Appl. Phys. Lett. 96(19), 192114 (2010).

${ }^{74}$ K. S. Chang, M. L. Green, I. Levin, and S. De Gendt, Scripta Materialia 68(5), 333-336 (2013).

${ }^{75} \mathrm{Y}$. Xu, Ferro-electric Materials and Their Applications (Elsevier, North Holland, 1991).

${ }^{76}$ N. Setter, D. Damjanovic, L. Eng, G. Fox, S. Gevorgian, S. Hong, A. Kingon, H. Kohlstedt, N. Y. Park, G. B. Stephenson, I. Stolitchnov, A. K. Taganstev, D. V. Taylor, T. Yamada, and S. Streiffer, J. Appl. Phys. 100(5), 051606 (2006).

${ }^{77}$ S. A. Wilson, R. P. J. Jourdain, Q. Zhang, R. A. Dorey, C. R. Bowen, M. Willander, Q. U. Wahab, M. A.-h. Safaa, O. Nur, E. Quandt, C. Johansson, E. Pagounis, M. Kohl, J. Matovic, B. Samel, W. van der Wijngaart, E. W. H. Jager, D. Carlsson, Z. Djinovic, M. Wegener, C. Moldovan, R. Iosub, E. Abad, M. Wendlandt, C. Rusu, and K. Persson, Mater. Sci. Eng., R 56(1-6), 1-129 (2007).

${ }^{78}$ G. Srinivasan, in Annual Review of Materials Research, edited by D. R. R. M. Z. F. Clarke (2010), Vol. 40, pp. 153-178.

${ }^{79}$ H. Chang, C. Gao, I. Takeuchi, Y. Yoo, J. Wang, P. G. Schultz, X. D. Xiang, R. P. Sharma, M. Downes, and T. Venkatesan, Appl. Phys. Lett. 72(17), 2185-2187 (1998).

${ }^{80}$ H. Y. Chang, K. M. Yu, Y. Dong, and X. D. Xiang, Appl. Phys. Lett. 81(11), 2062-2064 (2002).

${ }^{81}$ C. Gao, B. Hu, I. Takeuchi, K. S. Chang, X. D. Xiang, and G. Wang, Meas. Sci. Technol. 16(1), 248-260 (2005).

${ }^{82}$ W. Chang, J. S. Horwitz, W.-J. Kim, J. M. Pond, S. W. Kirchoeffer, and D. B. Chrisey, in Materials Research Society Meeting, edited by R. E. Jones, R. W. Schwartz, S. R. Summerfelt, and I. K. Yoo (MRS, Warrendale, PA, 1999), Vol. 541, pp. 699.

${ }^{83}$ J. C. Booth, I. Takeuchi, and K. S. Chang, Appl. Phys. Lett. 87(8), 082908 (2005) 
${ }^{84}$ H. Minami, K. Itaka, P. Ahmet, D. Komiyama, T. Chikyow, M. Lippmaa, and H. Koinuma, Jpn. J. Appl. Phys., Part 2 41(2A), L149-L151 (2002).

${ }^{85}$ K. S. Chang, M. Aronova, O. Famodu, I. Takeuchi, S. E. Lofland, J. Hattrick-Simpers, and H. Chang, Appl. Phys. Lett. 79(26), 4411-4413 (2001).

${ }^{86}$ G. He, T. Iijima, and H. Funakubo, J. Ceram. Soc. Jpn. 117(1365), 698-702 (2009).

${ }^{87}$ K. W. Kim, M. K. Jeon, K. S. Oh, T. S. Kim, Y. S. Kim, and S. I. Woo, Proc. Natl. Acad. Sci. U.S.A. 104(4), 1134-1139 (2007).

${ }^{88}$ M. Murakami, S. Fujino, S. H. Lim, L. G. Salamanca-Riba, M. Wuttig, I. Takeuchi, B. Varughese, H. Sugaya, T. Hasegawa, and S. E. Lofland, Appl. Phys. Lett. 88(11), 112505 (2006).

${ }^{89}$ S. Fujino, M. Murakami, V. Anbusathaiah, S. H. Lim, V. Nagarajan, C. J. Fennie, M. Wuttig, L. Salamanca-Riba, and I. Takeuchi, Appl. Phys. Lett. 92(20), 202904 (2008).

${ }^{90}$ G. Catalan and J. F. Scott, Adv. Mater. 21(24), 2463-2485 (2009).

${ }^{91}$ Y. M. Jin, Y. U. Wang, A. G. Khachaturyan, J. F. Li, and D. Viehland, Phys. Rev. Lett. 91(19), 197601-1-197601-4 (2003).

${ }^{92}$ D. Kan, L. Palova, V. Anbusathaiah, C. J. Cheng, S. Fujino, V. Nagarajan, K. M. Rabe, and I. Takeuchi, Adv. Funct. Mater. 20(7), 1108-1115 (2010).

${ }^{93}$ D. Kan, R. Suchoski, S. Fujino, and I. Takeuchi, Integr. Ferroelectr. 111, 116-124 (2009).

${ }^{94}$ J. L. Jones, A. Pramanick, and J. E. Daniels, Appl. Phys. Lett. 93(15), 152904 (2008)

${ }^{95}$ J. E. Daniels, W. Jo, J. Roedel, V. Honkimaeki, and J. L. Jones, Acta Mater. 58(6), 2103-2111 (2010).

${ }^{96}$ W. Hu, X. Tan, and K. Rajan, Appl. Phys. A: Mater. Sci. Process. 99(2), 427-431 (2010).

${ }^{97}$ C. A. F. Vaz, J. Hoffman, C. H. Anh, and R. Ramesh, Adv. Mater. 22(26-27), 2900-2918 (2010).

${ }^{98}$ M. Murakami, K. S. Chang, M. A. Aronova, C. L. Lin, M. H. Yu, J. H. Simpers, M. Wuttig, I. Takeuchi, C. Gao, B. Hu, S. E. Lofland, L. A. Knauss, and L. A. Bendersky, Appl. Phys. Lett. 87(11), 112901 (2005).

${ }^{99}$ K. S. Chang, M. A. Aronova, C. L. Lin, M. Murakami, M. H. Yu, J. Hattrick-Simpers, O. O. Famodu, S. Y. Lee, R. Ramesh, M. Wuttig, I. Takeuchi, C. Gao, and L. A. Bendersky, Appl. Phys. Lett. 84(16), 3091-3093 (2004)

${ }^{100}$ C. Gao, B. Hu, X. F. Li, C. H. Liu, M. Murakami, K. S. Chang, C. J. Long, M. Wuttig, and I. Takeuchi, Appl. Phys. Lett. 87(15), 153505 (2005).

${ }^{101}$ N. M. Aimon, D. H. Kim, H. K. Choi, and C. A. Ross, Appl. Phys. Lett. 100(9), 092901 (2012).

${ }^{102}$ J. M. D. Coey, Magnetism and Magnetic Materials (Cambridge University Press, Cambridge, UK, 2010).

${ }^{103}$ M. H. Yu, J. Hattrick-Simpers, I. Takeuchi, J. Li, Z. L. Wang, J. P. Liu, S. E. Lofland, S. Tyagi, J. W. Freeland, D. Giubertoni, M. Bersani, and M. Anderle, J. Appl. Phys. 98(6), 063908 (2005).

${ }^{104}$ T. Fukumura, M. Ohtani, M. Kawasaki, Y. Okimoto, T. Kageyama, T. Koida, T. Hasegawa, Y. Tokura, and H. Koinuma, Appl. Phys. Lett. 77(21), 3426-3428 (2000).

${ }^{105}$ Y. K. Yoo and F. Tsui, MRS Bull. 27(4), 316-323 (2002).

${ }^{106}$ Y. K. Yoo, T. Ohnishi, G. Wang, F. Duewer, X. D. Xiang, Y. S. Chu, D. C. Mancini, Y. Q. Li, and R. C. O'Handley, Intermetallics 9(7), 541-545 (2001).

${ }^{107}$ O. O. Famodu, J. Hattrick-Simpers, M. Aronova, K. S. Chang, M. Murakami, M. Wuttig, T. Okazaki, Y. Furuya, L. A. Knauss, L. A. Bendersky, F. S. Biancaniello, and I. Takeuchi, Mater. Trans. 45(2), 173-177 (2004).

${ }^{108}$ I. Takeuchi, O. O. Famodu, J. C. Read, M. A. Aronova, K. S. Chang, C. Craciunescu, S. E. Lofland, M. Wuttig, F. C. Wellstood, L. Knauss, and A. Orozco, Nature Mater. 2(3), 180-184 (2003).

${ }^{109} \mathrm{M}$. Aronova, University of Maryland, 2000.

${ }^{110}$ Y. Matsumoto, H. Koinuma, T. Hasegawa, I. Takeuchi, F. Tsui, and Y. K. Yoo, MRS Bull. 28(10), 734-739 (2003).

${ }^{111}$ R. Takahashi, H. Kubota, M. Murakami, Y. Yamamoto, Y. Matsumoto, and H. Koinuma, J. Comb. Chem. 6(1), 50-53 (2004).

${ }^{112}$ Y. Yamamoto, R. Takahashi, Y. Matsumoto, T. Chikyow, and H. Koinuma, Appl. Surf. Sci. 223(1-3), 9-13 (2004).

${ }^{113}$ Y. K. Yoo, F. Duewer, T. Fukumura, H. T. Yang, D. Yi, S. Liu, H. Y. Chang, T. Hasegawa, M. Kawasaki, H. Koinuma, and X. Xiang, Phys. Rev. B 63(22), 224421 (2001).

${ }^{114}$ R. Maezono, S. Ishihara, and N. Nagaosa, Phys. Rev. B 58(17), 11583-11596 (1998)
${ }^{115}$ M. J. Turchinskaya, L. A. Bendersky, A. J. Shapiro, K. S. Chang, I. Takeuchi, and A. L. Roytburd, J. Mater. Res. 19(9), 2546-2548 (2004).

${ }^{116}$ Y. Iwasaki, T. Fukumura, H. Kimura, A. Ohkubo, T. Hasegawa, Y. Hirose, T. Makino, K. Ueno, and M. Kawasaki, Appl. Phys. Express 3(10), 103001 (2010)

${ }^{117}$ R. A. Dunlap, J. M. Gaudet, and T. D. Hatchard, J. Magn. Magn. Mater. 320(21), 2730-2736 (2008).

${ }^{118}$ Y. Matsumoto, M. Murakami, T. Shono, T. Hasegawa, T. Fukumura, M. Kawasaki, P. Ahmet, T. Chikyow, S. Koshihara, and H. Koinuma, Science 291(5505), 854-856 (2001).

${ }^{119} \mathrm{P}$. Murugan, R. V. Belosludov, H. Mizuseki, T. Nishimatsu, T. Fukumura, M. Kawasaki, and Y. Kawazoe, Meas. Sci. Technol. 16(1), 242-247 (2005).

${ }^{120}$ Y. Yamada, T. Fukumura, M. Ikeda, M. Ohtani, H. Toyosaki, A. Ohtomo, F. Matsukura, H. Ohno, and M. Kawasaki, J. Supercond. 18(1), $109-113$ (2005).

${ }^{121}$ J. J. Hanak and J. I. Gittleman, AIP Conf. Proc. 10, 961-965 (1972).

${ }^{122}$ R. B. Vandover, M. Hong, E. M. Gyorgy, J. F. Dillon, and S. D. Albiston, J. Appl. Phys. 57(8), 3897-3899 (1985).

${ }^{123}$ D. Priyadarshini, P. Kondratyuk, J. B. Miller, and A. J. Gellman, J. Vac. Sci. Technol. A 30(1), 011503 (2012).

${ }^{124}$ Y. K. Yoo, Q. Z. Xue, Y. S. Chu, S. F. Xu, U. Hangen, H. C. Lee, W. Stein, and X. D. Xiang, Intermetallics 14(3), 241-247 (2006).

${ }^{125}$ R. M. Bozorth, Ferromagnetism (Wiley-IEEE Press, New Jersey, 1993).

${ }^{126}$ R. A. Degroot, F. M. Mueller, P. G. Vanengen, and K. H. J. Buschow, Phys. Rev. Lett. 50(25), 2024-2027 (1983).

${ }^{127}$ I. Takeuchi, O. O. Famodu, M. Aronova, and J. Hattrick-Simpers, in Proc. of the Eighth Intl. Symp. on Sputtering \& Plasma Proc. (Japan, 2005), p. 201.

${ }^{128}$ V. A. Chernenko, E. Cesari, V. V. Kokorin, and I. N. Vitenko, Scr. Metall. Mater. 33(8), 1239-1244 (1995).

${ }^{129}$ S. J. Murray, M. Marioni, P. G. Tello, S. M. Allen, and R. C. O'Handley, J. Magn. Magn. Mater. 226, 945-947 (2001).

${ }^{130}$ M. Wuttig, L. H. Liu, K. Tsuchiya, and R. D. James, J. Appl. Phys. 87(9), 4707-4711 (2000).

${ }^{131}$ C. Wedel and K. Itagaki, J. Phase Equilib. 22(3), 324-330 (2001).

${ }^{132}$ F. Tsui and P. A. Ryan, Appl. Surf. Sci. 189(3-4), 333-338 (2002).

${ }^{133}$ P. K. Muduli, W. C. Rice, L. He, B. A. Collins, Y. S. Chu, and F. Tsui, J. Phys. Condens. Matter 21(29), 296005 (2009).

${ }^{134}$ L. He, B. A. Collins, F. Tsui, and Y. S. Chu, J. Vac. Sci. Technol. B 29(3), 03 C124 (2011).

${ }^{135}$ J.-C. Zhao, M. R. Jackson, L. A. Peluso, and L. N. Brewer, MRS Bull. 27(4), 324-329 (2002)

${ }^{136}$ J. C. Zhao, Prog. Mater. Sci. 51(5), 557-631 (2006).

${ }^{137}$ J. C. Zhao, J. Mater. Sci. 39(12), 3913-3925 (2004).

${ }^{138}$ S. Huxtable, D. G. Cahill, V. Fauconnier, J. O. White, and J. C. Zhao, Nature Mater. 3(5), 298-301 (2004).

${ }^{139}$ X. Zheng, D. G. Cahill, and J.-C. Zhao, Acta Mater. 58(4), 1236-1241 (2010).

${ }^{140}$ J.-C. Zhao, X. Zheng, and D. G. Cahill, JOM 63(3), 40-44 (2011).

${ }^{141}$ J.-C. Zhao, in Methods for Phase Diagram Determination (Elsevier, Amsterdam, 2007), pp. 246-272.

${ }^{142}$ J. Unguris, R. J. Celotta, and D. T. Pierce, Phys. Rev. Lett. 67(1), 140-143 (1991).

${ }^{143}$ R. J. Celotta, D. T. Pierce, and J. Unguris, MRS Bull. 20(10), 30-33 (1995).

${ }^{144}$ K. Yosida, Phys. Rev. 106(5), 893-898 (1957).

${ }^{145}$ M. A. Ruderman and C. Kittel, Phys. Rev. 96(1), 99-102 (1954).

${ }^{146}$ M. J. Kramer, R. W. McCallum, A. Anderson, and S. Constantinides, J. Met. 64(7), 752-763 (2012).

${ }^{147}$ T. R. Gao, Y. Q. Wu, S. Fackler, I. Kierzewski, Y. Zhang, A. Mehta, M. J. Kramer, and I. Takeuchi, Appl. Phys. Lett. 102(2), 022419 (2013).

${ }^{148}$ E. F. Kneller and R. Hawig, IEEE Trans. Magn. 27(4), 3588-3600 (1991).

${ }^{149}$ E. E. Fullerton, J. S. Jiang, and S. D. Bader, J. Magn. Magn. Mater. 200(1-3), 392-404 (1999).

${ }^{150}$ E. E. Fullerton, J. S. Jiang, M. Grimsditch, C. H. Sowers, and S. D. Bader, Phys. Rev. B 58(18), 12193-12200 (1998).

${ }^{151}$ A. J. Zambano, H. Oguchi, I. Takeuchi, Y. Choi, J. S. Jiang, J. P. Liu, S. E. Lofland, D. Josell, and L. A. Bendersky, Phys. Rev. B 75(14), 144429 (2007).

${ }^{152}$ A. G. Khachaturyan and D. Viehland, Metall. Mater. Trans. A 38(13), 2317-2328 (2007). 
${ }^{153}$ J. R. Hattrick-Simpers, D. Hunter, C. M. Craciunescu, K. S. Jang, M. Murakami, J. Cullen, M. Wuttig, I. Takeuchi, S. E. Lofland, L. Benderksy, N. Woo, R. B. Van Dover, T. Takahashi, and Y. Furuya, Appl. Phys. Lett. 93(10), 102507 (2008).

${ }^{154}$ D. Hunter, W. Osborn, K. Wang, N. Kazantseva, J. Hattrick-Simpers, R. Suchoski, R. Takahashi, M. L. Young, A. Mehta, L. A. Bendersky, S. E. Lofland, M. Wuttig, and I. Takeuchi, Nat. Commun. 2, 2-7 (2011).

${ }^{155}$ J. R. Hattrick-Simpers, C. Jun, M. Murakami, A. Orozco, L. Knauss, R. J. Booth, E. W. Greve, S. E. Lofland, M. Wuttig, and I. Takeuchi, Appl. Surf. Sci. 254(3), 734-737 (2007).

${ }^{156}$ J. G. Bednorz and K. A. Muller, Z. Phys. B 64(2), 189-193 (1986).

${ }^{157}$ J. A. Clayhold, B. M. Kerns, M. D. Schroer, D. W. Rench, G. Logvenov, A. T. Bollinger, and I. Bozovic, Rev. Sci. Instrum. 79(3), 033908 (2008).

${ }^{158}$ K. C. Hewitt, P. A. Casey, R. J. Sanderson, M. A. White, and R. Sun, Rev. Sci. Instrum. 76(9), 093906 (2005).

${ }^{159}$ B. Knigge, A. Hoffmann, D. Lederman, D. C. Vier, S. Schultz, and I. K. Schuller, J. Appl. Phys. 81(5), 2291-2295 (1997).

${ }^{160}$ D. Lederman, D. C. Vier, D. Mendoza, J. Santamaria, S. Schultz, and I. K. Schuller, Appl. Phys. Lett. 66(26), 3677-3679 (1995).

${ }^{161}$ G. Logvenov, I. Sveklo, and I. Bozovic, Phys. C 460, 416-419 (2007).

${ }^{162}$ M. Saadat, A. E. George, and K. C. Hewitt, Phys. C 470, S59-S61 (2010).

${ }^{163}$ S. Pessaud, F. Gervais, C. Champeaux, P. Marchet, A. Catherinot, M. Licheron, J. L. Longuet, and F. Ravel, Mater. Sci. Eng., B 60(3), 205-211 (1999).

${ }^{164}$ J. Paglione and R. L. Greene, Nat. Phys. 6(9), 645-658 (2010).

${ }^{165}$ W. Wong-Ng, M. Otani, I. Levin, P. Schenck, Z. Yang, G. Liu, L. P. Cook, R. Feenstra, W. Zhang, and M. W. Rupich, Appl. Phys. Lett. 94(17), 171910 (2009).

${ }^{166} \mathrm{~J}$. Apte and Arasteh, D., 2011.

${ }^{167}$ K. M. Brace, B. E. Hayden, A. E. Russell, and J. R. Owen, Adv. Mater. 18(24), 3253 (2006).

${ }^{168}$ M. Soltani, M. Chaker, E. Haddad, R. V. Kruzelecky and J. Margot, Appl. Phys. Lett. 85(11), 1958-1960 (2004).

${ }^{169}$ P. Jin and S. Tanemura, Jpn. J. Appl. Phys., Part 1 34(5A), 2459-2460 (1995).

${ }^{170}$ W. Burkhardt, T. Christmann, S. Franke, W. Kriegseis, D. Meister, B. K. Meyer, W. Niessner, D. Schalch, and A. Scharmann, Thin Solid Films 402(1-2), 226-231 (2002).

${ }^{171}$ N. D. Bassim, P. K. Schenck, E. U. Donev, E. J. Heilweil, E. Cockayne, M. L. Green, and L. C. Feldman, Appl. Surf. Sci. 254(3), 785-788 (2007)

${ }^{172}$ K. Fujimoto, K. Onoda, M. Sato, H. Matsuo, T. Yamaguchi, and S. Ito, Mater. Sci. Eng., A 475(1-2), 52-56 (2008).

${ }^{173}$ X. D. Sun and X. D. Xiang, Appl. Phys. Lett. 72(5), 525-527 (1998).

${ }^{174}$ J. S. Wang, Y. Yoo, C. Gao, I. Takeuchi, X. D. Sun, H. Y. Chang, X. D. Xiang, and P. G. Schultz, Science 279(5357), 1712-1714 (1998).

${ }^{175}$ X. D. Sun, K. A. Wang, Y. Yoo, W. G. Wallace-Freedman, C. Gao, X. D. Xiang, and P. G. Schultz, Adv. Mater. 9(13), 1046 (1997).

${ }^{176}$ E. Danielson, M. Devenney, D. M. Giaquinta, J. H. Golden, R. C. Haushalter, E. W. McFarland, D. M. Poojary, C. M. Reaves, W. H. Weinberg, and X. D. Wu, Science 279(5352), 837-839 (1998).

${ }^{177}$ X. D. Sun, C. Gao, J. S. Wang, and X. D. Xiang, Appl. Phys. Lett. 70(25), 3353-3355 (1997).

${ }^{178}$ E. Danielson, J. H. Golden, E. W. McFarland, C. M. Reaves, W. H. Weinberg, and X. D. Wu, Nature 389(6654), 944-948 (1997).

${ }^{179}$ X.-D. Sun, P. G. Schultz, C. Gao, J. Wang, and X.-D. Xiang, Appl. Phys. Lett. 70(25), 3353-3355 (1997).

${ }^{180}$ E. D. Isaacs, M. Marcus, G. Aeppli, X. D. Xiang, X. D. Sun, P. Schultz, H. K. Kao, G. S. Cargill, and R. Haushalter, Appl. Phys. Lett. 73(13), 1820-1822 (1998).

${ }^{181}$ Y. Deng, J. D. Fowlkes, P. D. Rack, and J. M. Fitz-Gerald, Opt. Mater. 29(2-3), 183-191 (2006).

${ }^{182}$ Y. Deng, Y. F. Guan, and P. D. Rack, Thin Solid Films 515(4), $1721-1726$ (2006)

${ }^{183}$ A. Stein, S. W. Keller, and T. E. Mallouk, Science 259(5101), 1558-1564 (1993).

${ }^{184}$ V. Z. Mordkovich, H. Hayashi, M. Haemori, T. Fukumura, and M. Kawasaki, Adv. Funct. Mater. 13(7), 519-524 (2003).

${ }^{185}$ T. X. Sun and G. E. Jabbour, MRS Bull. 27(4), 309-315 (2002).

${ }^{186}$ Y. Yoshioka and G. E. Jabbour, Abstr. Pap. -Am. Chem. Soc. 227, U561-U562 (2004).

${ }^{187}$ Y. Yoshioka, P. D. Calvert, and G. E. Jabbour, Macromol. Rapid Commun. 26(4), 238-246 (2005).
${ }^{188}$ J. Perrière, E. Millon, and V. Craciun, in Pulsed Laser Deposition of Thin Films: Applications-Led Growth of Functional Materials, edited by R. Eason (John Wiley \& Sons, Inc., 2007).

${ }^{189}$ Y. Matsumoto, M. Murakami, Z. W. Jin, A. Ohtomo, M. Lippmaa, M. Kawasaki, and H. Koinuma, Jpn. J. Appl. Phys., Part 2 38(6AB), L603-L605 (1999).

${ }^{190}$ I. Takeuchi, W. Yang, K. S. Chang, M. A. Aronova, T. Venkatesan, R. D. Vispute, and L. A. Bendersky, J. Appl. Phys. 95(7), 3840-3840 (2004).

${ }^{191}$ I. Takeuchi, W. Yang, K. S. Chang, M. A. Aronova, T. Venkatesan, R. D. Vispute, and L. A. Bendersky, J. Appl. Phys. 94(11), 7336-7340 (2003).

${ }^{192}$ E. Fortunato, D. Ginley, H. Hosono, and D. C. Paine, MRS Bull. 32(3), 242-247 (2007).

${ }^{193}$ Q. Wang, J. Perkins, H. M. Branz, J. Alleman, C. Duncan, and D. Ginley, Appl. Surf. Sci. 189(3-4), 271-276 (2002).

${ }^{194}$ L. P. Yu and A. Zunger, Phys. Rev. Lett. 108(6), 068701 (2012).

${ }^{195}$ T. R. Paudel, A. Zakutayev, S. Lany, M. d'Avezac, and A. Zunger, Adv. Funct. Mater. 21(23), 4493-4501 (2011).

${ }^{196}$ A. Zakutayev, T. R. Paudel, P. F. Ndione, J. D. Perkins, S. Lany, A. Zunger, and D. S. Ginley, Phys. Rev. B 85(8), 085204 (2012).

${ }^{197}$ S. J. Henderson, J. A. Armstrong, A. L. Hector, and M. T. Weller, J. Mater. Chem. 15(15), 1528-1536 (2005)

${ }^{198}$ J. D. Perkins, M. P. Taylor, M. van Hest, C. W. Teplin, J. L. Alleman, M. S. Dabney, L. M. Gedvilas, B. M. Keyes, B. To, D. W. Readey, A. E. Delahoy, S. Guo, D. S. Ginley, and IEEE, in Conference Record of the Thirty-First IEEE Photovoltaic Specialists Conference-2005 (IEEE, New York, 2005), pp. 145-147.

${ }^{199}$ M. P. Taylor, D. W. Readey, C. W. Teplin, M. van Hest, J. L. Alleman, M. S. Dabney, L. M. Gedvilas, B. M. Keyes, B. To, P. A. Parilla, J. D. Perkins, and D. S. Ginley, Macromol. Rapid Commun. 25(1), 344-347 (2004).

${ }^{200}$ M. van Hest, M. S. Dabney, J. D. Perkins, D. S. Ginley, and M. P. Taylor, Appl. Phys. Lett. 87(3), 032111 (2005).

${ }^{201}$ M. P. Taylor, D. W. Readey, C. W. Teplin, M. van Hest, J. L. Alleman, M. S. Dabney, L. M. Gedvilas, B. M. Keyes, B. To, J. D. Perkins, and D. S. Ginley, Meas. Sci. Technol. 16(1), 90-94 (2005).

${ }^{202}$ G. S. Heo, Y. Matsumoto, I. G. Gim, J. W. Park, G. Y. Kim, and T. W. Kim, Jpn. J. Appl. Phys. 49(3), 035801 (2010).

${ }^{203}$ T. Koida and M. Kondo, J. Appl. Phys. 101(6), 063713 (2007).

${ }^{204}$ D. W. Sheel, H. M. Yates, P. Evans, U. Dagkaldiran, A. Gordijn, F. Finger, Z. Remes, and M. Vanecek, Thin Solid Films 517(10), 3061-3065 (2009).

${ }^{205}$ Z. Remes, M. Vanecek, H. M. Yates, P. Evans, and D. W. Sheel, Thin Solid Films 517(23), 6287-6289 (2009).

${ }^{206}$ H. M. Yates, P. Evans, D. W. Sheel, Z. Remes, and M. Vanecek, Thin Solid Films 519(4), 1334-1340 (2010).

${ }^{207}$ U. Dagkaldiran, A. Gordijn, F. Finger, H. M. Yates, P. Evans, D. W. Sheel, Z. Remes, and M. Vanecek, Mater. Sci. Eng., B 159-160, 6-9 (2009).

${ }^{208}$ J. D. Perkins, J. A. del Cueto, J. L. Alleman, C. Warmsingh, B. M. Keyes, L. M. Gedvilas, P. A. Parilla, B. To, D. W. Readey, and D. S. Ginley, Thin Solid Films 411(1), 152-160 (2002).

${ }^{209}$ A. Kurz and M. A. Aegerter, J. Sol-Gel Sci. Technol. 31(1-3), 267-271 (2004).

${ }^{210}$ C. W. Gorrie, M. Reese, J. D. Perkins, M. van Hest, J. L. Alleman, M. S. Dabney, B. To, D. S. Ginley, and J. J. Berry, in Pvsc: 2008 33rd IEEE Photovoltaic Specialists Conference (IEEE, New York, 2008), Vol. 1-4, pp. 635-637.

${ }^{211}$ K. Jung, W. K. Choi, S. J. Yoon, H. J. Kim, and J. W. Choi, Appl. Surf. Sci. 256(21), 6219-6223 (2010).

${ }^{212}$ I. T. Jolliffe, Principal Component Analysis, 2nd ed. (Springer verlag, New York, 2002).

${ }^{213}$ D. A. Kukuruznyak, P. Ahmet, T. Chikyow, A. Yamamoto, and F. S. Ohuchi, Appl. Surf. Sci. 252(10), 3828-3832 (2006).

${ }^{214}$ T. L. Chen, Y. Furubayashi, Y. Hirose, T. Hitosugi, T. Shimada, and T. Hasegawa, J. Phys. D: Appl. Phys. 40(19), 5961-5964 (2007).

${ }^{215}$ X. A. Li, T. A. Gessert, and T. Coutts, Appl. Surf. Sci. 223(1-3), $138-143$ (2004).

${ }^{216}$ S. Raoux and M. Wuttig, Phase Change Materials: Science and Applications (Springer-Verlag, 2008).

${ }^{217}$ J. Feinleib, J. Deneufvi, S. C. Moss, and S. Ovshinsky, Appl. Phys. Lett. 18(6), 254 (1971)

${ }^{218}$ M. Wuttig and N. Yamada, Nature Mater. 6(11), 824-832 (2007).

${ }^{219}$ D. Ielmini and A. L. Lacaita, Mater. Today 14(12), 600-607 (2011).

${ }^{220}$ R. G. Neale, D. L. Nelson, and G. E. Moore, Electronics 43(20), 56 (1970). 
${ }^{221}$ S. Ovshinsky, Phys. Rev. Lett. 21(20), 1450 (1968).

${ }^{222}$ N. Yamada, MRS Bull. 21(9), 48-50 (1996).

${ }^{223}$ N. Yamada, E. Ohno, K. Nishiuchi, N. Akahira, and M. Takao, J. Appl. Phys. 69(5), 2849-2856 (1991).

${ }^{224}$ S. Kyrsta, R. Cremer, D. Neuschutz, M. Laurenzis, P. H. Bolivar, and H. Kurz, Thin Solid Films 398, 379-384 (2001).

${ }^{225}$ M. Laurenzis, A. Heinrici, P. H. Bolivar, H. Kurz, S. Krysta, and J. M. Schneider, IEE Proc. Sci. Meas. Technol. 151(6), 394-397 (2004).

${ }^{226}$ M. G. Kanatzidis, S. D. Mahanti, and T. P. Hogan, Chemistry, Physics, and Materials Science of Thermoelectric Materials: Beyond Bismuth Telluride (Plenum Publishers, New York, NY, 2003).

${ }^{227}$ D. M. Rowe, in CRC Handbooks (CRC Press, Boca Raton, FL, 1995).

${ }^{228}$ H. Minami, K. Itaka, H. Kawaji, Q. J. Wang, H. Koinuma, and M. Lippmaa, Appl. Surf. Sci. 197, 442-447 (2002).

${ }^{229}$ M. Otani, N. D. Lowhorn, P. K. Schenck, W. Wong-Ng, M. L. Green, K. Itaka, and H. Koinuma, Appl. Phys. Lett. 91, 132102 (2007).

${ }^{230}$ M. Otani, E. L. Thomas, W. Wong-Ng, P. K. Schenck, K. S. Chang, N. D. Lowhorn, M. L. Green, and H. Ohguchi, Jpn. J. Appl. Phys. 48(5), 05EB02 (2009).

${ }^{231}$ R. Funahashi, S. Urata, and M. Kitawaki, Appl. Surf. Sci. 223(1-3), 44-48 (2004).

${ }^{232}$ R. Funahashi, M. Mikami, S. Urata, M. Kitawaki, T. Kouuchi, and K. Mizuno, Meas. Sci. Technol. 16(1), 70-80 (2005).

${ }^{233}$ M. Watanabe, T. Kita, T. Fukumura, A. Ohtomo, K. Ueno, and M. Kawasaki, J. Comb. Chem. 10(2), 175-178 (2008).

${ }^{234}$ A. Yamamoto, H. Obara, and K. Ueno, in MRS Fall Meeting, edited by T. P. Hogan, J. Yang, R. Funahashi and T. Tritt (Materials Research Society, Boston, 2007), Vol. 1044, pp. 273-278.

${ }^{235}$ X. Zheng, D. G. Cahill, P. Krasnochtchekov, R. S. Averback, and J. C. Zhao, Acta Mater. 55, 5177-5185 (2007).

${ }^{236}$ R. Marom, S. F. Amalraj, N. Leifer, D. Jacob, and D. Aurbach, J. Mater. Chem. 21(27), 9938-9954 (2011).

${ }^{237}$ W.-J. Zhang, J. Power Sources 196(1), 13-24 (2011).

${ }^{238}$ M. D. Fleischauer, T. D. Hatchard, A. Bonakdarpour, and J. R. Dahn, Meas. Sci. Technol. 16(1), 212-220 (2005).

${ }^{239}$ M. D. Fleischauer, J. M. Topple, and J. R. Dahn, Electrochem. Solid State Lett. 8(2), A137-A140 (2005)

${ }^{240}$ E. Reddington, A. Sapienza, B. Gurau, R. Viswanathan, S. Sarangapani, E. S. Smotkin, and T. E. Mallouk, Science 280(5370), 1735-1737 (1998).

${ }^{241} \mathrm{~K}$. Fujimoto, T. Kato, S. Ito, S. Inoue, and M. Watanabe, Solid State Ion. 177(26-32), 2639-2642 (2006).

${ }^{242}$ M. D. Fleischauer, T. D. Hatchard, G. P. Rockwell, J. M. Topple, S. Trussler, S. K. Jericho, M. H. Jericho, and J. R. Dahn, J. Electrochem. Soc. 150(11), A1465-A1469 (2003).

${ }^{243}$ M. G. Sullivan, H. Utomo, P. J. Fagan, and M. D. Ward, Anal. Chem. 71(19), 4369-4375 (1999).

${ }^{244}$ M. D. Fleischauer and J. R. Dahn, J. Electrochem. Soc. 151(8), A1216-A1221 (2004)

${ }^{245}$ K. Takada, K. Fujimoto, T. Sasaki, and M. Watanabe, Appl. Surf. Sci. 223(1-3), 210-213 (2004).

${ }^{246}$ J. F. Whitacre, W. C. West, and B. V. Ratnakumar, J. Electrochem. Soc. 150(12), A1676-A1683 (2003).

${ }^{247}$ A. D. Spong, G. Vitins, S. Guerin, B. E. Hayden, A. E. Russell, and J. R. Owen, J. Power Sources 119, 778-783 (2003).

${ }^{248}$ K. Suzuki, Y. Kuroiwa, S. Takami, M. Kubo, and A. Miyamoto, Appl. Surf. Sci. 189(3-4), 313-318 (2002)

${ }^{249}$ K. Fujimoto, K. Onoda, and S. Ito, Appl. Surf. Sci. 254(3), 704-708 (2007)

${ }^{250}$ K. Fujimoto, K. Takada, T. Sasaki, and M. Watanabe, Appl. Surf. Sci. 223(1-3), 49-53 (2004).

${ }^{251}$ G. Ceder, MRS Bull. 35(9), 693-701 (2010).

${ }^{252}$ M. K. Aydinol, A. F. Kohan, G. Ceder, K. Cho, and J. Joannopoulos, Phys. Rev. B 56(3), 1354-1365 (1997).

${ }^{253}$ G. Ceder, Y. M. Chiang, D. R. Sadoway, M. K. Aydinol, Y. I. Jang, and B. Huang, Nature 392(6677), 694-696 (1998).

${ }^{254}$ K. S. Kang, Y. S. Meng, J. Breger, C. P. Grey, and G. Ceder, Science 311(5763), 977-980 (2006).

${ }^{255} \mathrm{M}$. Roberts and J. Owen, ACS Comb. Sci. 13(2), 126-134 (2011).

${ }^{256}$ M. R. Roberts, G. Vitins, G. Denuault, and J. R. Owen, J. Electrochem. Soc. 157(4), A381-A386 (2010)

${ }^{257}$ T. D. Hatchard, J. R. Dahn, S. Trussler, M. Fleischauer, A. Bonakdarpour, J. R. Mueller-Neuhaus, and K. C. Hewitt, Thin Solid Films 443(1-2), 144-150 (2003).
${ }^{258}$ K. Dini and R. A. Dunlap, J. Phys. F: Met. Phys. 16(12), 1917-1925 (1986).

${ }^{259}$ A. Inoue, Y. Bizen, H. M. Kimura, T. Masumoto, and M. Sakamoto, J. Mater. Sci. 23(10), 3640-3647 (1988).

${ }^{260}$ Z. B. Sun, X. D. Wang, X. P. Li, M. S. Zhao, Y. Li, Y. M. Zhu, and X. P. Song, J. Power Sources 182(1), 353-358 (2008).

${ }^{261}$ A. D. W. Todd, R. E. Mar, and J. R. Dahn, J. Electrochem. Soc. 154(6), A597-A604 (2007).

${ }^{262}$ A. D. W. Todd, R. E. Mar, and J. R. Dahn, J. Electrochem. Soc. 153(10), A1998-A2005 (2006).

${ }^{263}$ J. Hassoun, G. Mulas, S. Panero, and B. Scrosati, Electrochem. Commun. 9(8), 2075-2081 (2007).

${ }^{264}$ Z. X. Chen, J. F. Qian, X. P. Ai, Y. H. Cao, and H. X. Yang, J. Power Sources 189(1), 730-732 (2009).

${ }^{265}$ M. A. Al-Maghrabi, J. S. Thorne, R. J. Sanderson, J. N. Byers, J. R. Dahn, and R. A. Dunlap, J. Electrochem. Soc. 159(6), A711-A719 (2012).

${ }^{266}$ N. Tamura, R. Ohshita, M. Fujimoto, S. Fujitani, M. Kamino, and I. Yonezu, J. Power Sources 107(1), 48-55 (2002).

${ }^{267}$ M. Winter and J. O. Besenhard, Electrochim. Acta 45(1-2), 31-50 (1999).

${ }^{268}$ J. S. Thorne, R. J. Sanderson, J. R. Dahn, and R. A. Dunlap, J. Electrochem. Soc. 157(10), A1085-A1091 (2010).

${ }^{269}$ A. Zuettel, A. Remhof, A. Borgschulte, and O. Friedrichs, Philos. Trans. R. Soc. London, Ser. A 368(1923), 3329-3342 (2010).

${ }^{270}$ L. Schlapbach and A. Zuttel, Nature 414(6861), 353-358 (2001).

${ }^{271}$ C. Wu, G. Wu, Z. Xiong, X. Han, H. Chu, T. He, and P. Chen, Chem. Mater. 22(1), 3-5 (2010).

${ }^{272}$ J. E. Katz, T. R. Gingrich, E. A. Santori, and N. S. Lewis, Energy Environ. Sci. 2(1), 103-112 (2009).

${ }^{273}$ A. Stepanovich, K. Sliozberg, W. Schuhmann, and A. Ludwig, Int. J. Hydrogen Energy 37(16), 11618-11624 (2012).

${ }^{274}$ A. J. Leenheer and H. A. Atwater, J. Electrochem. Soc. 159(9), H752-H757 (2012).

${ }^{275}$ M. Woodhouse and B. A. Parkinson, Chem. Soc. Rev. 38(1), 197-210 (2009).

${ }^{276}$ I. P. Jain, P. Jain, and A. Jain, J. Alloys Compd. 503(2), 303-339 (2010).

${ }^{277}$ G. Sandrock, K. Gross, and G. Thomas, J. Alloys Compd. 339(1-2), 299-308 (2002).

${ }^{278} \mathrm{C}$. Weidenthaler, A. Pommerin, M. Felderhoff, B. Bogdanovic, and F. Schuth, Phys. Chem. Chem. Phys. 5(22), 5149-5153 (2003).

${ }^{279}$ J. J. Reilly and R. H. Wiswall, Inorg. Chem. 7(11), 2254 (1968).

${ }^{280}$ D. J. Siegel, C. Wolverton, and V. Ozolins, Phys. Rev. B 76(13), 134102 (2007).

${ }^{281}$ J. J. Vajo, S. L. Skeith, and F. Mertens, J. Phys. Chem. B 109(9), 3719-3722 (2005).

${ }^{282}$ P. Chen, Z. T. Xiong, J. Z. Luo, J. Y. Lin, and K. L. Tan, Nature 420(6913), 302-304 (2002).

${ }^{283}$ G. P. Meisner, M. L. Scullin, M. P. Balogh, F. E. Pinkerton, and M. S. Meyer, J. Phys. Chem. B 110(9), 4186-4192 (2006).

${ }^{284}$ Y. E. Filinchuk, K. Yvon, G. P. Meisner, F. E. Pinkerton, and M. P. Balogh, Inorg. Chem. 45(4), 1433-1435 (2006).

${ }^{285}$ F. E. Pinkerton, G. P. Meisner, M. S. Meyer, M. P. Balogh, and M. D. Kundrat, J. Phys. Chem. B 109(1), 6-8 (2005).

${ }^{286}$ W. F. Luo, J. Alloys Compd. 381(1-2), 284-287 (2004).

${ }^{287}$ J. Lu, Z. Z. Fang, Y. J. Choi, and H. Y. Sohn, J. Phys. Chem. C 111(32), 12129-12134 (2007).

${ }^{288}$ Y. Nakamori, G. Kitahara, K. Miwa, N. Ohba, T. Noritake, S. Towata, and S. Orimo, J. Alloys Compd. 404, 396-398 (2005).

${ }^{289}$ H. Y. Leng, T. Ichikawa, S. Hino, N. Hanada, S. Isobe, and H. Fujii, J. Phys. Chem. B 108(26), 8763-8765 (2004).

${ }^{290}$ H. Wu, W. Zhou, T. J. Udovic, J. J. Rush, and T. Yildirim, Chem. Mater. 20(4), 1245-1247 (2008).

${ }^{291}$ J. R. Hattrick-Simpers, J. E. Maslar, M. U. Niemann, C. Chiu, S. S. Srinivasan, E. K. Stefanakos, and L. A. Bendersky, Int. J. Hydrogen Energy 35(12), 6323-6331 (2010).

${ }^{292}$ S. S. Srinivasan, M. U. Niemann, J. R. Hattrick-Simpers, K. McGrath, P. C. Sharma, D. Y. Goswami, and E. K. Stefanakos, Int. J. Hydrogen Energy 35(18), 9646-9652 (2010).

${ }^{293}$ J. Yang, A. Sudik, D. J. Siegel, D. Halliday, A. Drews, R. O. Carter III, C. Wolverton, G. J. Lewis, J. W. A. Sachtler, J. J. Low, S. A. Faheem, D. A. Lesch, and V. Ozolins, Angew. Chem., Int. Ed. 47(5), 882-887 (2008).

${ }^{294}$ G. Kresse and J. Hafner, Phys. Rev. B 47(1), 558-561 (1993). 
${ }^{295}$ P. Villars and L. D. Calvert, Pearson's Handbook of Crystallographic Data for Intermetallic Phases, 2nd ed. (ASM International, Materials Park, Ohio, 1991).

${ }^{296}$ G. Bergerhoff and I. D. Brown, Crystallographic Databases, edited by F. H. Allen, G. Bergerhoff, and R. Sievers (International Union of Crystallography, Chester, UK, 1987), pp. 77-95.

${ }^{297}$ See http://www.fiz-karlsruhe.de/icsd.html (FIZ, Karlsruhe, Germany, 2012).

${ }^{298}$ S. V. Alapati, J. K. Johnson, and D. S. Sholl, J. Phys. Chem. B 110(17), 8769-8776 (2006)

${ }^{299}$ S. V. Alapati, J. K. Johnson, and D. S. Sholl, J. Phys. Chem. C 111(4), 1584-1591 (2007)

${ }^{300}$ S. V. Alapati, J. K. Johnson, and D. S. Sholl, Phys. Chem. Chem. Phys. 9(12), 1438-1452 (2007).

${ }^{301}$ S. V. Alapati, J. K. Johnson, and D. S. Sholl, J. Phys. Chem. C 112(14), 5258-5262 (2008)

${ }^{302}$ H.-W. Li, K. Miwa, N. Ohba, T. Fujita, T. Sato, Y. Yan, S. Towata, M. W. Chen, and S. Orimo, Nanotechnology 20(20), 204013 (2009).

${ }^{303}$ H.-W. Li, Y. Yan, S.-I. Orimo, A. Zuettel, and C. M. Jensen, Energies 4(1), 185-214 (2011).

${ }^{304}$ V. Ozolins, E. H. Majzoub, and C. Wolverton, J. Am. Chem. Soc. 131(1), 230-237 (2009).

${ }^{305}$ V. Ozolins, E. H. Majzoub, and C. Wolverton, Phys. Rev. Lett. 100(13), 135501 (2008)

${ }^{306}$ C. Wolverton, D. J. Siegel, A. R. Akbarzadeh, and V. Ozolins, J. Phys. Condens. Matter 20(6), 064228 (2008).

${ }^{307}$ A. R. Akbarzadeh, C. Wolverton, and V. Ozolins, Phys. Rev. B 79(18), 184102 (2009)

${ }^{308}$ W. Q. Sun, C. Wolverton, A. R. Akbarzadeh, and V. Ozolins, Phys. Rev. B 83(6), 064112 (2011).

${ }^{309}$ R. Gremaud, C. P. Broedersz, A. Borgschulte, M. J. van Setten, H. Schreuders, M. Slaman, B. Dam, and R. Griessen, Acta Mater. 58(2), 658-668 (2010).

${ }^{310}$ A. Ludwig, J. Cao, A. Savan, and M. Ehmann, J. Alloys Compd. 446, 516-521 (2007).

${ }^{311}$ N. C. Woo, B. G. Ng, and R. B. van Dover, Rev. Sci. Instrum. 78(7), 072208 (2007)

${ }^{312}$ J. R. Hattrick-Simpers, W. S. Hurst, S. S. Srinivasan, and J. E. Maslar, Rev. Sci. Instrum. 82(3), 033103 (2011).

${ }^{313}$ J. R. Hattrick-Simpers, K. Wang, L. Cao, C. Chiu, E. Heilweil, R. G. Downing, and L. A. Bendersky, J. Alloys Compd. 490(1-2), 42-46 (2010).

${ }^{314}$ G. Garcia, R. Domenech-Ferrer, F. Pi, J. Santiso, and J. Rodriguez-Viejo, J. Comb. Chem. 9(2), 230-236 (2007).

${ }^{315}$ C. H. Olk, in Materials and Technology for Hydrogen Economy, edited by G. A. Nazri, M. Nazri, R. Young, and P. Chen (Materials Research Society (MRS), 2004), Vol. 801, pp. 75-88.

${ }^{316}$ R. Gremaud, C. P. Broedersz, D. M. Borsa, A. Borgschulte, P. Mauron, H. Schreuders, J. H. Rector, B. Dam, and R. Griessen, Adv. Mater. 19(19), 2813 (2007)

${ }^{317}$ I. Giebels, J. Isidorsson, E. S. Kooij, A. Remhof, N. J. Koeman, J. H. Rector, A. T. M. van Gogh, and R. Griessen, J. Alloys Compd. 330, 875-881 (2002)

${ }^{318}$ C. H. Olk, G. G. Tibbetts, D. Simon, and J. J. Moleski, J. Appl. Phys. 94(1), 720-725 (2003).

${ }^{319}$ H. Oguchi, E. J. Heilweil, D. Josell, and L. A. Bendersky, J. Alloys Compd. 477(1-2), 8-15 (2009).

${ }^{320}$ H. Oguchi, J. Hattrick-Simpers, I. Takeuchi, E. J. Heilweil, and L. A. Bendersky, Rev. Sci. Instrum. 80(7), 8 (2009).

${ }^{321}$ H. Oguchi, Z. Tan, E. J. Heilweil, and L. A. Bendersky, Int. J. Hydrogen Energy 35(3), 1296-1299 (2010).

${ }^{322}$ J. N. Huiberts, R. Griessen, J. H. Rector, R. J. Wijnaarden, J. P. Dekker, D. G. deGroot, and N. J. Koeman, Nature 380(6571), 231-234 (1996).

${ }^{323}$ A. Borgschulte, W. Lohstroh, R. J. Westerwaal, H. Schreuders, J. H. Rector, B. Dam, and R. Griessen, J. Alloys Compd. 404, 699-705 (2005).

${ }^{324} \mathrm{~A}$. Remhof and A. Borgschulte, ChemPhysChem 9(17), 2440-2455 (2008).

${ }^{325}$ R. Gremaud, M. Slaman, H. Schreuders, B. Dam, and R. Griessen, Appl. Phys. Lett. 91(23), 231916 (2007).

${ }^{326}$ R. J. Westertwaal, C. den Besten, M. Slaman, B. Dam, D. E. Nanu, A. J. Bottger, and W. G. Haije, Int. J. Hydrogen Energy 36(1), 1074-1082 (2011).

${ }^{327}$ A. Baldi, D. M. Borsa, H. Schreuders, J. H. Rector, T. Atmakidis, M. Bakker, H. A. Zondag, W. G. J. van Helden, B. Dam, and R. Griessen, Int. J. Hydrogen Energy 33(12), 3188-3192 (2008).
${ }^{328}$ A. Borgschulte, R. Gremaud, S. de Man, R. J. Westerwaal, J. H. Rector, B. Dam, and R. Griessen, Appl. Surf. Sci. 253(3), 1417-1423 (2006).

${ }^{329}$ A. Borgschulte, J. H. Rector, H. Schreuders, B. Dam, and R. Griessen, Appl. Phys. Lett. 90(7), 071912 (2007).

${ }^{330}$ B. Dam, R. Gremaud, C. Broedersz, and R. Griessen, Scr. Mater. 56(10), $853-858$ (2007).

${ }^{331}$ R. Gremaud, J. L. M. van Mechelen, H. Schreuders, M. Slaman, B. Dam, and R. Griessen, Int. J. Hydrogen Energy 34(21), 8951-8957 (2009).

${ }^{332}$ C. P. Broedersz, R. Gremaud, B. Dam, R. Griessen, and O. M. Lovvik, Phys. Rev. B 77(2), 024204 (2008).

${ }^{333}$ R. Gremaud, A. Borgschulte, C. Chacon, J. L. M. Van Mechelen, H. Schreuders, A. Zuttel, B. Hjorvarsson, B. Dam, and R. Griessen, Appl. Phys. A 84(1-2), 77-85 (2006).

${ }^{334}$ R. Gremaud, A. Borgschulte, W. Lohstroh, H. Schreuders, A. Zuttel, B. Dam, and R. Griessen, J. Alloys Compd. 404, 775-778 (2005).

${ }^{335}$ E. Johansson, C. Chacon, C. Zlotea, Y. Andersson, and B. Hjorvarsson, J. Phys. Condens. Matter 16(43), 7649-7662 (2004).

${ }^{336}$ L. R. Cao, J. R. Hattrick-Simpers, R. Bindel, B. E. Tomlin, R. Zeisler, R. Paul, L. A. Bendersky, and R. G. Downing, J. Radioanal. Nucl. Chem. 283(1), 63-68 (2010).

${ }^{337}$ A. Ludwig, J. Cao, B. Dam, and R. Gremaud, Appl. Surf. Sci. 254(3), 682-686 (2007).

${ }^{338}$ G. J. Lewis, J. W. A. Sachtler, J. J. Low, D. A. Lesch, S. A. Faheem, P. M. Dosek, L. M. Knight, L. Halloran, C. M. Jensen, J. Yang, A. Sudik, D. J. Siegel, C. Wolverton, V. Ozolins, and S. Zhang, J. Alloys Compd. 446, 355-359 (2007).

${ }^{339}$ V. L. Smentkowski, J. P. Lemmon, and J. C. Zhao, in 205th Meeting, San Antonio, Texas (Electrochemical Society, 2004), Abstract 294.

${ }^{340}$ A. Sudik, J. Yang, D. J. Siegel, C. Wolverton, R. O. Carter III, and A. R. Drews, J. Phys. Chem. C 113(5), 2004-2013 (2009).

${ }^{341}$ A. Borgschulte, R. Gremaud, Z. Lodziana, and A. Zuettel, Phys. Chem. Chem. Phys. 12(19), 5061-5066 (2010).

${ }^{342}$ A. Borgschulte, R. Gremaud, S. Kato, N. P. Stadie, A. Remhof, A. Zuettel, M. Matsuo, and S. I. Orimo, Appl. Phys. Lett. 97(3), 031916 (2010).

${ }^{343}$ K. K. S. Singhal, High Temperature Solid Oxide Fuel Cells: Fundamentals, Design, and Applications (Elsevier, Oxford, UK, 2003).

${ }^{344}$ E. S. Smotkin and R. R. Diaz-Morales, Ann. Rev. Mater. Res. 33, 557-579 (2003).

${ }^{345}$ W. Z. Zhu and S. C. Deevi, Mater. Sci. Eng., A 362(1-2), 228-239 (2003).

${ }^{346}$ A. B. Stambouli and E. Traversa, Renewable Sustainable Energy Rev. 6(5), 433-455 (2002).

${ }^{347}$ H. S. Liu, C. J. Song, L. Zhang, J. J. Zhang, H. J. Wang, and D. P. Wilkinson, J. Power Sources 155(2), 95-110 (2006).

${ }^{348}$ V. Tripkovic, F. Abild-Pedersen, F. Studt, I. Cerri, T. Nagami, T. Bligaard, and J. Rossmeisl, ChemCatChem 4(2), 228-235 (2012).

${ }^{349}$ J. M. Gregoire, R. B. van Dover, J. Jin, F. J. DiSalvo, and H. D. Abruna, Rev. Sci. Instrum. 78(7), 072212 (2007).

${ }^{350}$ J. S. Cooper and P. J. McGinn, J. Power Sources 163(1), 330-338 (2006).

${ }^{351}$ K. Jambunathan, S. Jayaraman, and A. C. Hillier, Langmuir 20(5), 1856-1863 (2004).

${ }^{352}$ P. Strasser, J. Comb. Chem. 10(2), 216-224 (2008).

${ }^{353}$ W. C. Choi, J. D. Kim, and S. I. Woo, Catal. Today 74(3-4), 235-240 (2002).

${ }^{354}$ F. G. Welsch, K. Stoewe, and W. F. Maier, Catal. Today 159(1), 108-119 (2011).

${ }^{355}$ D. F. A. Koch, D. A. J. Rand, and R. Woods, J. Electroanal. Chem. 70(1), 73-86 (1976).

${ }^{356}$ C. Gaudillere, P. Vernoux, C. Mirodatos, G. Caboche, and D. Farrusseng, Catal. Today 157(1-4), 263-269 (2010).

${ }^{357}$ J. C. H. Rossiny, S. Fearn, J. A. Kilner, Y. Zhang, and L. Chen, Solid State Ion. 177(19-25), 1789-1794 (2006).

${ }^{358}$ B. Sung-Hyeon, T. F. Jaramillo, A. Kleiman-Shwarsctein, and E. W. McFarland, Meas. Sci. Technol. 16(1), 54 (2005).

${ }^{359}$ S. Jayaraman and A. C. Hillier, J. Comb. Chem. 6(1), 27-31 (2004).

${ }^{360}$ M. Prochaska, J. Jin, D. Rochefort, L. Zhuang, F. J. DiSalvo, H. D. Abruna, and R. B. van Dover, Rev. Sci. Instrum. 77(5), 054104 (2006).

${ }^{361}$ S. Jayaraman and A. C. Hillier, Meas. Sci. Technol. 16(1), 5-13 (2005).

${ }^{362}$ X. M. Lin, L. Y. Zheng, G. M. Gao, Y. W. Chi, and G. N. Chen, Anal. Chem. 84(18), 7700-7707 (2012).

${ }^{363}$ B. C. Shah and A. C. Hillier, J. Electrochem. Soc. 147(8), 3043-3048 (2000). 
${ }^{364}$ T. H. Muster, A. Trinchi, T. A. Markley, D. Lau, P. Martin, A. Bradbury, A. Bendavid, and S. Dligatch, Electrochim. Acta 56(27), 9679-9699 (2011).

${ }^{365}$ T. H. Muster, H. Sullivan, D. Lau, D. L. J. Alexander, N. Sherman, S. J. Garcia, T. G. Harvey, T. A. Markley, A. E. Hughes, P. A. Corrigan, A. M. Glenn, P. A. White, S. G. Hardin, J. Mardel, and J. M. C. Mol, Electrochim. Acta 67, 95-103 (2012).

${ }^{366}$ T. H. Muster, A. E. Hughes, S. A. Furman, T. Harvey, N. Sherman, S. Hardin, P. Corrigan, D. Lau, F. H. Scholes, P. A. White, M. Glenn, J. Mardel, S. J. Garcia, and J. M. C. Mol, Electrochim. Acta 54(12), 3402-3411 (2009).

${ }^{367}$ R. X. Liu and E. S. Smotkin, J. Electroanal. Chem. 535(1-2), 49-55 (2002).

${ }^{368}$ B. C. Chan, R. X. Liu, K. Jambunathan, H. Zhang, G. Y. Chen, T. E. Mallouk, and E. S. Smotkin, J. Electrochem. Soc. 152(3), A594-A600 (2005).

${ }^{369}$ R. Z. Jiang, C. Rong, and D. Chu, J. Comb. Chem. 7(2), 272-278 (2005).

${ }^{370}$ R. Z. Jiang and D. Chu, J. Electroanal. Chem. 527(1-2), 137-142 (2002).

${ }^{371}$ Y. Zhang and P. J. McGinn, J. Power Sources 206, 29-36 (2012).

${ }^{372}$ R. R. Diaz-Morales, R. X. Liu, E. Fachini, G. Y. Chen, C. U. Segre, A. Martinez, C. Cabrera, and E. S. Smotkin, J. Electrochem. Soc. 151(9), A1314-A1318 (2004).

${ }^{373}$ E. S. Smotkin, J. H. Jiang, A. Nayar, and R. X. Liu, Appl. Surf. Sci. 252(7), 2573-2579 (2006).

${ }^{374}$ J. Jin, M. Prochaska, D. Rochefort, D. K. Kim, L. Zhuang, F. J. DiSalvo, R. B. Van Dover, and H. D. Abruna, Appl. Surf. Sci. 254(3), 653-661 (2007).

${ }^{375} \mathrm{~K}$. Jambunathan and A. C. Hillier, J. Electrochem. Soc. 150(6), E312-E320 (2003).

${ }^{376}$ Y. H. Chu, Y. G. Shul, W. C. Choi, S. I. Woo, and H. S. Han, J. Power Sources 118(1-2), 334-341 (2003).

${ }^{377}$ S. Jayaraman and A. C. Hillier, J. Phys. Chem. B 107(22), 5221-5230 (2003).

${ }^{378}$ J. F. Whitacre, T. Valdez, and S. R. Narayanan, J. Electrochem. Soc. 152(9), A1780-A1789 (2005).

${ }^{379}$ T. Kobayashi, A. Ueda, Y. Yamada, and H. Shioyama, Appl. Surf. Sci. 223(1-3), 102-108 (2004)

${ }^{380}$ S. Guerin, B. E. Hayden, C. E. Lee, C. Mormiche, J. R. Owen, A. E. Russell, B. Theobald, and D. Thompsett, J. Comb. Chem. 6(1), 149-158 (2004).

${ }^{381}$ H.-J. Kim, D.-Y. Kim, H. Han, and Y.-G. Shul, J. Power Sources 159(1), 484-490 (2006)

${ }^{382}$ S. Jayaraman, S. H. Baeck, T. F. Jaramillo, A. Kleiman-Shwarsctein, and E. W. McFarland, Rev. Sci. Instrum. 76(6), 062227 (2005).

${ }^{383}$ J. M. Gregoire, M. Kostylev, M. E. Tague, P. F. Mutolo, R. B. van Dover, F. J. DiSalvo, and H. D. Abruna, J. Electrochem. Soc. 156(1), B160-B166 (2009).

${ }^{384}$ M. K. Jeon, C. H. Lee, G. I. Park, and K. H. Kang, J. Power Sources 216, 400-408 (2012).

${ }^{385}$ J. F. Whitacre, T. I. Valdez, and S. R. Narayanan, Electrochim. Acta 53(10), 3680-3689 (2008).

${ }^{386}$ G. Y. Chen, D. A. Delafuente, S. Sarangapani, and T. E. Mallouk, Catal. Today 67(4), 341-355 (2001).

${ }^{387}$ K. R. Lee, Y. Jung, and S. I. Woo, ACS Comb. Sci. 14(1), 10-16 (2012).

${ }^{388}$ S. P. Jiang and S. H. Chan, J. Mater. Sci. 39(14), 4405-4439 (2004).

${ }^{389}$ J. Beckers, C. Gaudillere, D. Farrusseng, and G. Rothenberg, Green Chem. 11(7), 921-925 (2009).

${ }^{390}$ J. C. H. Rossiny, J. Julis, S. Fearn, J. A. Kilner, Y. Zhang, L. Chen, S. Yang, and J. R. G. Evans, Solid State Ion. 179(21-26), 1085-1089 (2008).

${ }^{391}$ S. Fearn, J. C. H. Rossiny, J. A. Kilner, Y. Zhang, and L. Chen, Appl. Surf. Sci. 252(19), 7159-7162 (2006).

${ }^{392}$ J. P. Lemmon, V. Manivannan, T. Jordan, L. Hassib, O. Siclovan, M. Othon and M. Pilliod, paper presented at the MRS (Boston, 2003).

${ }^{393}$ T. Gebhardt, D. Music, T. Takahashi, and J. M. Schneider, Thin Solid Films 520(17), 5491-5499 (2012)

${ }^{394}$ P. C. Collins, Ph.D., Ohio State University, 2004, ProQuest Dissertations And Theses, Publication Number: AAI3144861, ISBN: 9780496086047.

${ }^{395}$ Y. S. Chu, A. Tkachuk, S. Vogt, P. Ilinski, D. A. Walko, D. C. Mancini, E. M. Dufresne, L. He, and F. Tsui, Appl. Surf. Sci. 223(1-3), 175-182 (2004)

${ }^{396}$ A. Rar, J. J. Frafjord, J. D. Fowlkes, E. D. Specht, P. D. Rack, M. L. Santella, H. Bei, E. P. George, and G. M. Pharr, Meas. Sci. Technol. 16(1), 46-53 (2005).

${ }^{397}$ J. M. Gregoire, D. Dale, A. Kazimirov, F. J. DiSalvo, and R. B. van Dover, Rev. Sci. Instrum. 80(12), 123905 (2009).
${ }^{398}$ S. Vogt, Y. S. Chu, A. Tkachuk, P. Ilinski, D. A. Walko, and F. Tsui, Appl. Surf. Sci. 223(1-3), 214-219 (2004).

${ }^{399}$ J. Sakurai, S. Hata, R. Yamauchi, and A. Shimokohbe, Jpn. J. Appl. Phys. Part 1 46(4A), 1590-1595 (2007).

${ }^{400}$ L. A. Baumes, M. Moliner, N. Nicoloyannis, and A. Corma, Cryst. Eng. Commun. 10(10), 1321-1324 (2008).

${ }^{401}$ J. M. Gregoire, D. Dale, and R. B. van Dover, Rev. Sci. Instrum. 82(1), 015105 (2011).

${ }^{402}$ C. J. Long, D. Bunker, X. Li, V. L. Karen, and I. Takeuchi, Rev. Sci. Instrum. 80(10), 103902 (2009).

${ }^{403}$ C. J. Long, J. Hattrick-Simpers, M. Murakami, R. C. Srivastava, I. Takeuchi, V. L. Karen, and X. Li, Rev. Sci. Instrum. 78(7), 072217 (2007).

${ }^{404}$ I. Takeuchi, C. J. Long, O. O. Famodu, M. Murakami, J. HattrickSimpers, G. W. Rubloff, M. Stukowski, and K. Rajan, Rev. Sci. Instrum. 76(6), 062223 (2005).

${ }^{405}$ G. Barr, W. Dong, and C. J. Gilmore, J. Appl. Crystallogr. 37, 658-664 (2004).

${ }^{406}$ V. Chevrier and J. R. Dahn, Meas. Sci. Technol. 17(6), 1399-1404 (2006).

${ }^{407}$ S. Ermon, R. Le Bras, C. Gomes, B. Selman, and R. B. van Dover, paper presented at the 15 th International Conference on Theory and Applications of Satisfiability Testing, Trento, Italy, 2012.

${ }^{408}$ D. Kan, C. J. Long, C. Steinmetz, S. E. Lofland, and I. Takeuchi, J. Mater. Res. 27(21), 2691-2704 (2012)

${ }^{409}$ See http://www.whitehouse.gov/blog/2012/05/14/new-commitmentssupport-administration-s-materials-genome-initiative for highlights of the Materials Genome Initiative (MGI), a program whose goal is to reduce the time and cost of high technology materials' discovery, development and commercialization (2012).

${ }^{410}$ S. Curtarolo, W. Setyawan, G. L. W. Hart, M. Jahnatek, R. V. Chepulskii, R. H. Taylor, S. Wang, J. Xue, K. Yang, O. Levy, M. J. Mehl, H. T. Stokes, D. O. Demchenko, and D. Morgan, Comput. Mater. Sci. 58, 218-226 (2012).

${ }^{411}$ R. V. Belosludov, S. Takami, M. Kubo, and A. Miyamoto, in Combinatorial Materials Synthesis, edited by X.-D. Xiang and I. Takeuchi (Marcel Dekker, New York, 2003).

${ }^{412}$ J. W. Bennett, K. F. Garrity, K. M. Rabe, and D. Vanderbilt, Phys. Rev. Lett. 109(16), 167602 (2012).

${ }^{413}$ K. S. Yang, W. Setyawan, S. D. Wang, M. B. Nardelli, and S. Curtarolo, Nature Mater. 11(7), 614-619 (2012).

${ }^{414}$ S. Curtarolo, G. L. W. Hart, M. B. Nardelli, N. Mingo, S. Sanvito, and O. Levy, Nature Mater. 12(3), 191-201 (2013).

${ }^{415}$ D. C. Lagoudas, Shape Memory Alloys: Modeling and Engineering Applications (Springer, New York, 2008).

${ }^{416}$ J. Cui, Y. S. Chu, O. O. Famodu, Y. Furuya, J. Hattrick-Simpers, R. D. James, A. Ludwig, S. Thienhaus, M. Wuttig, Z. Y. Zhang, and I. Takeuchi, Nature Mater. 5(4), 286-290 (2006).

${ }^{417}$ J. M. Ball and R. D. James, Philos. Trans. R. Soc. London, Ser. A 338(1650), 389-450 (1992).

${ }^{418}$ R. Zarnetta, R. Takahashi, M. L. Young, A. Savan, Y. Furuya, S. Thienhaus, B. Maass, M. Rahim, J. Frenzel, H. Brunken, Y. S. Chu, V. Srivastava, R. D. James, I. Takeuchi, G. Eggeler, and A. Ludwig, Adv. Funct. Mater. 20(12), 1917-1923 (2010).

${ }^{419}$ R. Zarnetta, A. Savan, S. Thienhaus, and A. Ludwig, Appl. Surf. Sci. 254(3), 743-748 (2007).

${ }^{420}$ R. Zarnetta, P. J. S. Buenconsejo, A. Savan, S. Thienhaus, and A. Ludwig, Intermetallics 26, 98-109 (2012).

${ }^{421}$ F. J. J. Vanloo, G. F. Bastin, and A. J. H. Leenen, J. Less-Common Met. 57(1), 111-121 (1978).

${ }^{422}$ A. Dwivedi, T. J. Wyrobek, O. L. Warren, J. Hattrick-Simpers, O. O. Famodu, and I. Takeuchi, J. Appl. Phys. 104(7), 073501 (2008).

${ }^{423}$ A. Ludwig, J. Cao, J. Brugger, and I. Takeuchi, Meas. Sci. Technol. 16(1), 111-118 (2005)

${ }^{424}$ Y. W. Lai, S. Hamann, M. Ehmann, and A. Ludwig, Rev. Sci. Instrum. 82(6), 063903 (2011)

${ }^{425}$ S. Burger, C. Eberl, A. Siegel, A. Ludwig, and O. Kraft, Sci. Technol. Adv. Mater. 12(5), 054202 (2011).

${ }^{426}$ H.-J. Kim, J.-H. Han, R. Kaiser, K. H. Oh, and J. J. Vlassak, Rev. Sci. Instrum. 79(4), 045112 (2008).

${ }^{427}$ P. J. McCluskey and J. J. Vlassak, Scr. Mater. 64(3), 264-267 (2011).

${ }^{428}$ S. Semancik, in Combinatorial Materials Synthesis, edited by X. D. Xiang and I. Takeuchi (Marcel Dekker, Inc., New York, 2003), p. 469.

${ }^{429}$ R. A. Potyrailo and V. M. Mirsky, Combinatorial Methods for Chemical and Biological Sensors (Springer, Berlin, 2011). 
${ }^{430}$ M. W. Barsoum, Prog. Solid State Chem. 28(1-4), 201-281 (2000).

${ }^{431}$ B. Manoun, S. K. Saxena, T. El-Raghy, and M. W. Barsoum, Appl. Phys. Lett. 88(20), 201902 (2006).

${ }^{432}$ B. Manoun, S. K. Saxena, and M. W. Barsoum, Appl. Phys. Lett. 86(10), 101906 (2005).

${ }^{433}$ S. E. Lofland, J. D. Hettinger, K. Harrell, P. Finkel, S. Gupta, M. W. Barsoum, and G. Hug, Appl. Phys. Lett. 84(4), 508-510 (2004).

${ }^{434}$ T. H. Scabarozi, S. Benjamin, B. Adamson, J. Applegate, J. Roche, E. Pfeiffer, C. Steinmetz, C. Lunk, M. W. Barsoum, J. D. Hettinger, and S. E. Lofland, Scr. Mater. 66(2), 85-88 (2012).
${ }^{435}$ T. H. Scabarozi, C. Gennaoui, J. Roche, T. Flemming, K. Wittenberger, P. Hann, B. Adamson, A. Rosenfeld, M. W. Barsoum, J. D. Hettinger, and S. E. Lofland, Appl. Phys. Lett. 95(10), 101907 (2009).

${ }^{436}$ T. X. Sun, in Combinatorial Materials Synthesis, edited by X. D. Xiang and I. Takeuchi (Marcel Dekker, New York, 2003), pp. 141-176.

${ }^{437}$ Y. Terada, K. Ohkubo, T. Mohri, and T. Suzuki, J. Appl. Phys. 81(5), 2263-2268 (1997).

${ }^{438}$ S. Eccarius, B. L. Garcia, C. Hebling, and J. W. Weidner, J. Power Sources 179(2), 723-733 (2008).

${ }^{439}$ P. J. McCluskey, C. Zhao, O. Kfir, and J. J. Vlassak, Acta Mater. 59(13), 5116-5124 (2011) 